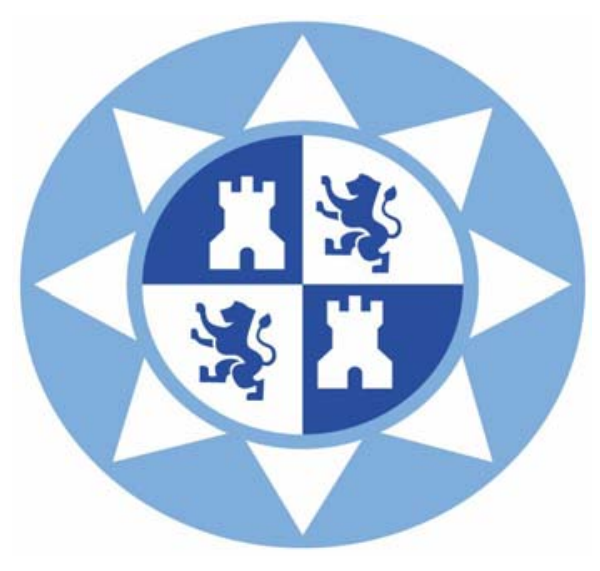

Universidad Politécnica de Cartagena

Departamento de Ingeniería de Sistemas y Automática

\title{
Reglas de Diseño para la Sintonía de Controladores Predictivos Dynamic Matrix Control (DMC)
}

Clemente Manzanera Reverter

\author{
Directores \\ Julio José Ibarrola Lacalle \\ José Manuel Cano Izquierdo
}




\section{Agradecimientos}

Lo primero que quiero hacer es agradecer como se merecen a todas las personas que han hecho posible la redacción de esta tesis doctoral. Sin su ayuda y apoyo no habría podido llegar tan lejos:

A mi familia (mis padres, mi hermano y mi abuela), por haberme animado desde el primer día. Sin su entusiasta apoyo, no estaría aquí.

A todos mis amigos, que siempre me han espoleado para que siguiera adelante, aunque eso significara tener que dedicarles menos tiempo. Una mención especial para aquellos que me retasteis a que iniciara este camino con vosotros.

A mis directores de tesis ( Dr.Julio Ibarrola y Dr. José Manuel Cano), cuya orientación y paciencia ha sido clave para finalizar este trabajo.

A la Jefa del Negociado de Postgrado y Doctorado, Marisa Rubio, por ayudarme con todos los trámites.

Y, en general, a todo el personal de la Universidad Politécnica de Cartagena que ha hecho posible la finalización de este trabajo.

Muchas gracias a todos. 


\section{Universidad \\ Politécnica \\ de Cartagena}

DT-16

\section{CONFORMIDAD DE SOLICITUD DE AUTORIZACIÓN DE DEPÓSITO DE}

\section{TESIS DOCTORAL POR EL/LA DIRECTOR/A DE LA TESIS}

D. Julio Ibarrola Lacalle/ José Manuel Cano Izquierdo Director/a de la Tesis doctoral REGLAS DE DISEÑO PARA LA SINTONÍA DE CONTROLADORES PREDICTIVOS DYNAMIC MATRIX CONTROL (DMC).

\section{INFORMA:}

Que la referida Tesis Doctoral, ha sido realizada por D. Clemente Manzanera Reverter, dentro del Programa de Doctorado Tecnologías Industriales, dando mi conformidad para que sea presentada ante el Comité de Dirección de la Escuela Internacional de Doctorado para ser autorizado su depósito.

La rama de conocimiento en la que esta tesis ha sido desarrollada es:

区 Ingeniería y Arquitectura

En Cartagena, a 20 de febrero de 2017

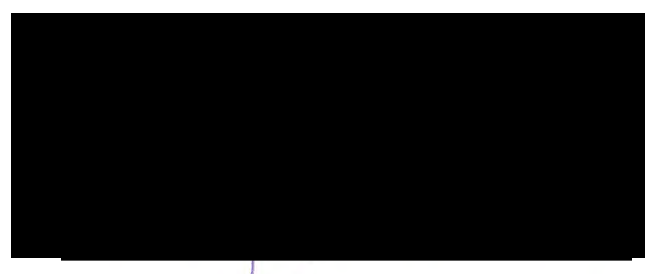

Fdo.: Julio José Ibarrola Lacalle

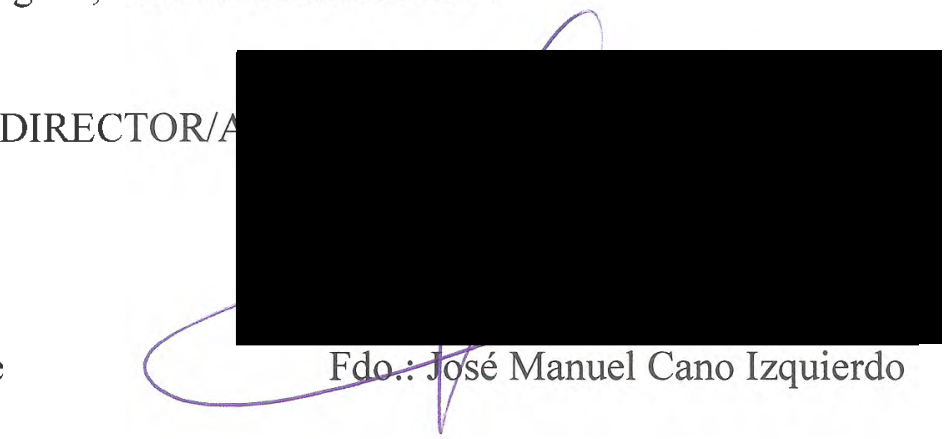




\section{Universidad \\ Politécnica \\ de Cartagena}

DT-17

\section{CONFORMIDAD DE DEPÓSITO DE TESIS DOCTORAL POR LA COMISIÓN ACADÉMICA DEL PROGRAMA}

D. Juan Suardíaz Muro, Presidente/a de la Comisión Académica del Programa Tecnologías Industriales.

\section{INFORMA:}

Que la Tesis Doctoral titulada," REGLAS DE DISEÑO PARA LA SINTONÍA DE CONTROLADORES PREDICTIVOS DYNAMIC MATRIX CONTROL (DMC)”, ha sido realizada, dentro del mencionado Programa de Doctorado, por D.Clemente Manzanera Reverter, bajo la dirección y supervisión del Dr./ Dra Julio Ibarrola Lacalle y José Manuel Cano Izquierdo.

En reunión de la Comisión Académica de fechaol/02/2017 visto que en la misma se acreditan los indicios de calidad correspondientes y la autorización del Director/a de la misma, se acordó dar la conformidad, con la finalidad de que sea autorizado su depósito por el Comité de Dirección de la Escuela Internacional de Doctorado.

La Rama de conocimiento por la que esta tesis ha sido desarrollada es:

I Ingeniería y Arquitectura

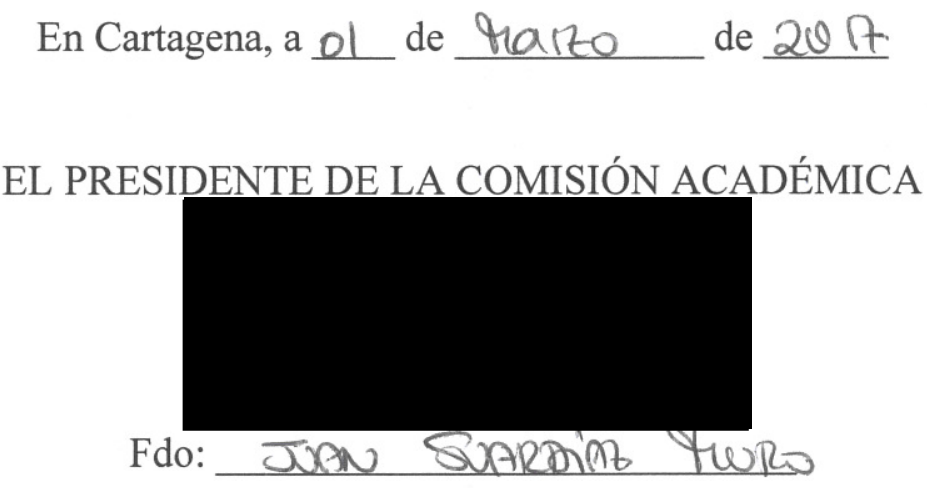




\section{Universidad}

Politécnica

de Cartagena

DT-18

\section{SOLICITUD DE AUTORIZACIÓN DE DEPÓSITO DE TESIS DOCTORAL POR EL DOCTORANDO/A}

\begin{tabular}{|c|l|}
\hline $\begin{array}{c}\text { TÍTULO DE LA } \\
\text { TESIS }\end{array}$ & $\begin{array}{l}\text { REGLAS DE DISEÑO PARA LA SINTONÍA DE CONTROLADORES } \\
\text { PREDICTIVOS DYNAMIC MATRIX CONTROL (DMC) }\end{array}$ \\
\hline DOCTORANDO/A & Clemente Manzanera Reverter \\
\hline
\end{tabular}

DATOS DE LA TESIS DOCTORAL

\begin{tabular}{|c|c|}
\hline Programa: & Tecnologías Industriales \\
\hline Titulación Acceso: & Ingeniero Industrial \\
\hline Director/a: & $\begin{array}{l}\text { Julio Ibarrola Lacalle } \\
\text { José Manuel Cano Izquierdo }\end{array}$ \\
\hline $\begin{array}{l}\text { Rama de Conocimiento } \text { (Ciencias, } \\
\text { Ciencias Sociales y Jurídicas, } \\
\text { Ingeniería y Arquitectura) }\end{array}$ & Ingeniería y Arquitectura \\
\hline $\begin{array}{l}\text { ¿Opta por Compendio de } \\
\text { Publicaciones? (Sí/No) }\end{array}$ & No \\
\hline $\begin{array}{l}\text { ¿Opta a la Mención Internacional? } \\
(\mathrm{Sí} / \mathrm{No})\end{array}$ & No \\
\hline $\begin{array}{l}\text { ¿Concurre alguna circunstancia de } \\
\text { confidencialidad que impida } \\
\text { publicación en abierto en } \\
\text { repositorio digital? (art. } 33.2 \text { del } \\
\text { Reglamento). Detállese. }\end{array}$ & No \\
\hline $\begin{array}{l}\text { Fecha de aprobación del Plan de } \\
\text { Investigación: }\end{array}$ & Mayo 2014 \\
\hline
\end{tabular}

El doctorando/a deberá aportar la siguiente documentación de acuerdo con el art. 24.5 del Reglamento de Estudios Oficiales de Doctorado de la UPCT:

$\square$ Un borrador de la tesis doctoral.

$\square$ Autorizaciones de depósito por parte de la Comisión Académica del programa y del director(es) de tesis y, en su caso, del tutor/a. 


\section{Universidad Politécnica de Cartagena}

$\square$ Indicio de calidad (art. 21 del Reglamento de Estudios Oficiales de Doctorado de la UPCT).

Además de la documentación anteriormente mencionada, el doctorando/a presentará asimismo los siguientes documentos en los casos que se indican a continuación:

\section{SI SE OPTA POR LA MODALIDAD TESIS POR COMPENDIO DE PUBLICACIONES}

$\square$ Copia de los artículos o trabajos publicados (o aceptados) que conformarán la tesis doctoral (siempre trabajos posteriores al inicio de los estudios de doctorado), acreditando el factor de impacto de las revistas, medios de referencia en los que hayan sido publicados y, en el caso de las patentes, copia de la solicitud, concesión y explotación, según sea el caso.

$\square$ Informe del Director/a de tesis justificando su presentación como tesis doctoral como compendio de publicaciones.

$\square$ Escrito en el que el Director/a y el codirector/a, en su caso, y con el visto bueno del Coordinador del Programa de Doctorado, declaren su conformidad con la presentación de la citada tesis por parte del doctorando/a, así como que la aportación de éste/a en los artículos que componen la tesis ha sido relevante.

$\square$ En el caso de la participación de otros autores/as distintos al director/a, y codirector/a en su caso, se requerirá además renuncia expresa por parte de cada uno/a a usar los citados artículos o patentes en otro depósito de tesis.

\section{SI SE OPTA A LA MENCIÓN INTERNACIONAL}

(Programas de doctorado regidos por el RD 99/2011)

$\square$ Certificado de estancia fuera de España (mínima de 3 meses) en una universidad o centro de investigación de prestigio en una institución de enseñanza superior o Centro de Investigación de prestigio, cursando estudios o realizando trabajos de investigación.

$\square$ Informe por un mínimo de dos expertos doctores/as pertenecientes a alguna institución de educación superior o Instituto de investigación no español. En dichos informes deberá constar la idoneidad de la tesis con el objetivo de acceder al grado de Doctor/a.

$\square$ El resumen, objetivos y conclusiones, redactados y presentados en una de las lenguas habituales para la comunicación científica en su campo de conocimiento, distinta a cualquiera de las lenguas oficiales en España. Esta norma no será de aplicación cuando las estancias, informes y expertos procedan de un país de habla hispana. 


\section{Universidad}

Politécnica

de Cartagena

DT-18

SOLICITA: Que tras comprobar que, la tesis presentada, cumple todos los requisitos establecidos por la legislación y reglamentación vigente, sea admitida a trámite de depósito con la finalidad de proceder con posterioridad a la defensa de ésta.

Cartagena, a 20 de febrero de 2017

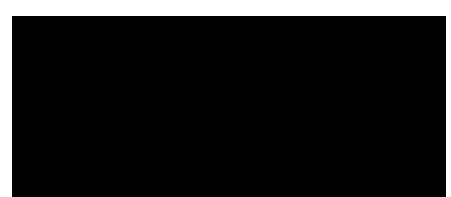

Firma del doctorando/a 
Universidad

Politécnica

de Cartagena

DT-18

COMITÉ DE DIRECCIÓN ESCUELA INTERNACIONAL DE DOCTORADO 
Dynamic Matrix Control (DMC) has widely expanded in Industry in the last 30 years. Despite this success, this control technique still lacks of a set of design rules for the choice of tuning parameters with the requirements of the practitioners. These are: easy to carry out, without the need of an exhaustive knowledge of the dynamics beyond the step tests, but able to provide an acceptable performance as startup tuning.

To achieve this, an exhaustive study of the closed loop system poles and zeros (using DMC controller) has been made as an indicator of the time response. The transformation of the system using the LTI equivalent has allowed the development of a set of tuning rules that fulfill the required characteristics for SISO and MIMO plants.

In the case of MIMO systems it has been necessary to develop in this work a specific formulation that allows obtaining the closed loop equivalent.

Following the results obtained for the DMC proposed tuning rules, the method has been put to test studying DMC control performance in some cases: Simulation of several SISO and MIMO widely used benchmarks extracted from the bibliography, control of a real thermal system, simulation of highly non-linear MIMO system. In all cases there has been a performance comparison with some already used techniques with successful results improving existing methods.

The set of provided rules matches with the required characteristics: They are easy to understand and use, they do not require advanced knowledge of mathematics and control theory and provide an acceptable Controlled Output for a start up first tuning. 
El control dinámico matricial nació a finales de la década de los 70 como respuesta a necesidades específicas de la industria de procesos que los métodos de de control de aquella época no podían ofrecer: Un control multivariable que tuviera en cuenta restricciones. Desde entonces se ha expandido con fuerza en la industria. Sin embargo, a pesar de este éxito, esta técnica de control aún carece de un método claro de sintonía con las características que demandan los usuarios: Que sea fácil de utilizar, apto para multivaraible,que no necesite un extenso conocimiento de la planta más allá del modelo de respuesta a escalón (necesario para usar el DMC) y que proporcione unas prestaciones aceptables que sirvan como base para un ajuste en detalle.

Para conseguir este objetivo, se ha optado por utilizar una técnica propia de la teoría de sistemas: Un exhaustivo análisis del efecto de los parámetros del DMC en los polos y ceros en lazo cerrado. Para ello ha sido necesario poder expresar una planta controlada por un algoritmo en un sistema de bloques mediante un equivalente LTI, que convierte al DMC en un sistema de bloques en lazo cerrado del que se puedan extraer funciones de transferencia y, consecuentemente, polos y ceros.

Este equivalente LTI se ha empleado tanto para sistemas SISO como MIMO. La base para sistemas SISO de este equivalente ya existe en la literatura. Pero para sistemas MIMO ha sido necesario desarrollar una formulación específica en este trabajo que permita obtener el equivalente en lazo cerrado para llevar a cabo el estudio de polos y ceros. Sin embargo, la utilidad de este equivalente no se limita al cálculo de polos y ceros, sino que es una útil herramienta que puede ser utilizada en futuros estudios del DMC como los de estabilidad y robustez. Como ejemplo de su utilidad, se ha usado para demostrar que el DMC actúa como un desacoplador para sistemas multivariable.

El uso de esta herramienta de la teoría de sistemas con el DMC ha permitido obtener una serie de reglas de diseño que han sido puestas a prueba de varias formas: Mediante simulación del control DMC de varios benchmark (SISO y MIMO) extraídos de la literatura, mediante el control DMC por simulación de un modelo altamente no lineal previamente usado por otros investigadores en sus trabajos y mediante el control de una maqueta térmica (un sistema físico real)

En los casos anteriores, el método propuesto ha sido comparado con otras técnicas de ajuste existentes, obteniéndose resultados que demuestran que el método obtenido supone una mejora respecto a los existentes.

Los resultados demuestran que las reglas desarrolladas cumplen con los objetivos fijados: Son sencillas de usar y entender, son válidas tanto para sistemas MIMO como SISO, no requieren un conocimiento del sistema más allá del necesario para usar el DMC (solo el modelo de respuesta a escalón), no es necesario poseer fuertes conocimientos matemáticos ni de teoría de de control para comprenderlas y proporcionan un control aceptable como un primer ajuste que puede ser refinado posteriormente. 


\section{Índice general}

1. Objetivos 3

1.1. Introducción . . . . . . . . . . . . . . . . . . 3

1.1.1. Ajuste de parámetros . . . . . . . . . . . . . . 4

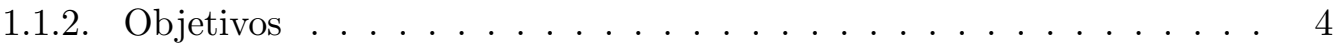

1.1.3. Estructura y metodología . . . . . . . . . . . . . 5

2. Introducción al control predictivo $\quad 7$

2.1. Introducción . . . . . . . . . . . . . . . . . . 7

2.2. La historia del MPC en la industria . . . . . . . . . . . . . . . . . . . . . . . . . 9

2.2.1. La fase cero. El regulador LQG. . . . . . . . . . . . . . . . . 9

2.2.2. La primera generación. DMC y MAC . . . . . . . . . . . . . 11

2.2.3. La segunda generación. QDMC . . . . . . . . . . . . . . . . . . . . 11

2.2.4. La tercera generación . . . . . . . . . . . . . . . . . . . . 12

2.2.5. La cuarta generación. . . . . . . . . . . . . . . . . 14

2.3. Estrategia del MPC . . . . . . . . . . . . . . . . . 14

2.4. Métodos de control predictivo . . . . . . . . . . . . . 16

2.4.1. Tecnologías Industriales . . . . . . . . . . . . . . . . . . . 22

2.5. Ajuste de parámetros del MPC . . . . . . . . . . . . . . . . . 24

2.5.1. Resumen de parámetros del MPC . . . . . . . . . . . . . 24

2.5.2. Estado del arte del ajuste de parámetros . . . . . . . . . . . 25

3. Reglas de diseño para sistemas SISO

3.1. Introducción . . . . . . . . . . . . . . . . . 27

3.2. Formulación LTI para DMC en el caso SISO . . . . . . . . . . . . . . 27

3.3. Polos en lazo cerrado y su efecto en la respuesta temporal . . . . . . . . . . 29

3.4. Influencia de los parámetros del DMC en la respuesta del sistema . . . . . . 30

3.4.1. Influencia del horizonte de predicción y el tiempo de muestreo en

la respuesta temporal. . . . . . . . . . . . . . 33

3.4.2. Influencia del horizonte de control en la respuesta temporal. . . . . . 36

3.4.3. Influencia del factor de peso en la respuesta temporal . . . . . . . . 39

3.4.4. Influencia del horizonte de modelo en la respuesta temporal . . . . . 41

3.4.5. Estabilidad del sistema . . . . . . . . . . . . . . . . . . 43

3.5. Reglas de diseño preliminares . . . . . . . . . . . . . . . . 45 
3.6. Validación del método . . . . . . . . . . . . . . . . . 47

3.6.1. Bechmarks . . . . . . . . . . . . . . . . . 47

3.6.2. Resultados . . . . . . . . . . . . . . . . . . 48

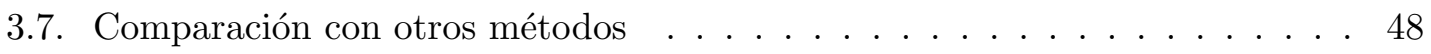

3.8. Prueba en una maqueta térmica . . . . . . . . . . . . . 51

3.9. Conclusiones . . . . . . . . . . . . . . . . . 58

4. Formulación LTI para sistemas MIMO 59

4.1. Introducción . . . . . . . . . . . . . . . . . . . . . . . . 59

4.2. Desarrollo del DMC como un sistema LTI MIMO para el caso [2 x 2] . . . 60

4.2.1. Diagrama de bloques LTI . . . . . . . . . . . . . . . 62

4.2.2. Matriz de transferencia LTI . . . . . . . . . . . . . . . . 66

4.3. Generalización a más variables . . . . . . . . . . . . . . . 70

4.4. Validación . . . . . . . . . . . . . . . . . . . . . 72

4.5. DMC como desacoplador . . . . . . . . . . . . . . . . 73

4.6. Conclusiones . . . . . . . . . . . . . . . . . . . 78

5. Reglas de diseño para sistemas MIMO $\quad 81$

5.1. Introducción . . . . . . . . . . . . . . . . . . 81

5.2. Influencia del horizonte de predicción . . . . . . . . . . . . . . 81

5.3. Influencia del horizonte de control . . . . . . . . . . . . . . . . . . . . . . . . . . . . . . . . 82

5.4. Influencia del factor de peso . . . . . . . . . . . . . . . . . . 82

5.5. Reglas de diseño para sistemas MIMO . . . . . . . . . . . . . . 96

5.6. Validación de reglas de diseño para sistemas MIMO . . . . . . . . . . . 97

5.6.1. Benchmark $1 \ldots \ldots \ldots$. . . . . . . . . . . . . . . . . . . . . . . . . . .

5.6.2. Benchmark $2 \ldots \ldots \ldots \ldots$. . . . . . . . . . . . 99

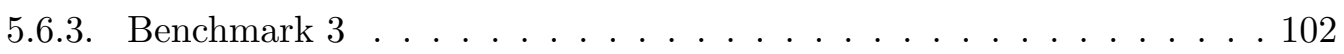

5.7. Comparación con el método de Shridhar Coopper . . . . . . . . . . . . . . 102

5.8. Columna de destilación de alta pureza . . . . . . . . . . . . . 106

5.8.1. Modelo de la columna de destilación de alta pureza . . . . . . . . . . 111

5.8.2. Control mediante DMC . . . . . . . . . . . . . . 116

5.9. Análisis de resultados . . . . . . . . . . . . . . . . . . . 121

$\begin{array}{ll}\text { 6. Conclusiones } & 123\end{array}$

A. Simplificaciones para formulación LTI MIMO $\quad 125$ 


\section{Capítulo 1}

\section{Objetivos}

\subsection{Introducción}

El control predictivo apareció en la industria a finales de la década de los setenta para cumplir las necesidades específicas de la industria petroquímica y centrales eléctricas [1], que no se podían satisfacer con el tradicional controlador PID. Durante décadas dicho controlador había dominado las aplicaciones de control de procesos debido a su sencillez, robustez, el completo conocimiento del mismo y el hecho que pudiera ser ajustado para que fuera estable [2]. Sin embargo su sencillez traía consigo varias limitaciones importantes:

- Es un método monovariable. En plantas con múltiples variables (como la mayoría de plantas reales) esto obliga a controlar cada subproceso independientemente de los demás, despreciando la interacción entre ellos, o desacoplando lazos.

- No tiene en cuenta las restricciones

Debido a estas limitaciones era necesario encontrar nuevos métodos de control, y a finales de los años 70 aparecieron varios trabajos que comenzaban a definir el control predictivo:

- Richalet et al [3], [4] presentaban el Control algorítmico por modelo (Model Algorith Control, MAC).

- Cutler y Ramaker [5] presentaban el Control Dinámico Matricial (Dynamic matrix Control, DMC).

Ambos métodos usan un modelo dinámico del proceso para predecir el efecto de las futuras acciones de control en la salida.

El control predictivo se volvió popular en la industria química rápidamente. Los principales motivos fueron la sencillez del algoritmo y el uso de modelos de respuesta a escalón o impulso que eran más intuitivos y requerían menos información a priori de la planta que los modelos matemáticos. Sin embargo a pesar de su éxito estos algoritmos adolecían de 
teorías formales que proporcionaran estabilidad o robustez. De hecho, el caso del horizonte finito era demasiado complicado de resolver, salvo en casos especiales.

\subsubsection{Ajuste de parámetros}

Una de las líneas de investigación que existen actualmente es la relacionada con la robustez y estabilidad del MPC. Pueden citarse los trabajos de Dubay et al ([6],[7]), que desarrollaron una nueva y más robusta formulación para el DMC, y de Webber y Gupta [8] que analizan las condiciones nominales de robustez y estabilidad para el MPC. Además, destacan los trabajos de Clarke [9], Mosca [10] y Kouvaritakis et al [11] mencionados anteriormente.

Sin embargo, a pesar de que exista una línea de investigación dedicada a la robustez y la estabilidad, en la práctica estas se consiguen mediante el ajuste de parámetros. El ajuste del MPC es otra importante línea de investigación. Inicialmente el ajuste de estos parámetros se hacía mediante prueba y error, llegándose a proponer algunos procedimientos, [12]. Marchetti [13] presentó un análisis de sensibilidad de los distintos parámetros a ajustar y su efecto en las prestaciones del DMC.

La dificultad de ajustar los parámetros del DMC es la cantidad que tiene (sobretodo en casos multivariable), cada uno de ellos con su efecto en la respuesta temporal. Algunos de ellos, como se verá más adelante, tienen efectos complementarios mientras que otros parecen actuar de manera antagónica y cada uno de ellos presenta un rango de valores útiles limitado. El primer paso es decidir sobre que parámetros incidir.

\subsubsection{Objetivos}

Teniendo en cuenta lo expuesto anteriormente, se presenta como objetivo del presente trabajo el desarrollo de una serie de reglas de ajuste para Control Predictivo basado en Modelo (MPC). Este trabajo se centrará en el método más popular de esta familia de controladores, el Control Dinámico Matricial (DMC).

Tal y como se ha explicado en el apartado 1.1.1 con más detalle, a pesar de su enorme desarrollo en las tres últimas décadas, el control predictivo sigue adoleciendo de un método de sintonía universal y sencillo, sobre todo para MIMO. Algo en la línea de los métodos experimentales ya establecidos para PID a nivel industrial. A lo largo de los años se han desarrollado varios métodos de sintonía pero pocos han llegado a ser aceptados de forma universal. Además, la mayoría de ellos has sido diseñados para sistemas monovariable cuando una de las grandes ventajas del DMC es su capacidad para controlar sistemas multivariables

Este objetivo puede desglosarse de la siguiente forma: 
1. Se formulará una herramienta que posibilite un estudio sistemático del DMC, comenzando por el caso más sencillo, el monovariable. Para ello se transformará el el algoritmo del DMC en un sistema de bloques de funciones de transferencia al que se le pueden aplicar las técnicas clásicas de análisis de sistemas. Las bases de este método pueden encontrarse en [14] para sistemas SISO.

2. Una vez se consiga dicha herramienta se utilizará para conseguir un método de ajuste para sistemas SISO controlados con DMC. Este método de ajuste deberá ser sencillo de utilizar y no necesitará del usuario fuertes conocimientos de teoría de control, pero tendrá que posibilitar un control con unas prestaciones adecuadas.

3. Se desarrollará la herramienta del equivalente LTI para poder aplicarla a sistema MIMO.

4. Por último, se utilizarán los resultados de los capítulos anteriores para confeccionar una serie de reglas de diseño para sistemas MIMO controlados por DMC. Al igual que para sistemas SISO, se pretende que la metodología sea simple de usar sin que sea necesario sacrificar prestaciones.

\subsubsection{Estructura y metodología}

Este trabajo está estructurado de la siguiente manera:

- En primer lugar se hará una introducción en la que se explicarán los fundamentos del MPC, la historia del MPC, los algoritmos más populares y el estado actual del arte indicando las líneas de investigación existentes en las que es más interesante incidir.

- En el tercer capítulo se desarrollará un método original para el ajuste de los parámetros del DMC basado en datos de simulaciones y en el estudio del movimiento de polos y ceros en el plano z, de forma similar al estudio realizado en [15]. Para esto será necesario expresar el algoritmo del DMC como un sistema LTI. Dichas reglas serán validadas en varios benchmarks extraídos de la literatura, comparadas con otros métodos existentes y puestas a prueba en una maqueta real. Siguiendo una metodología habitual ([16], [17], [18]), estas reglas de sintonía se construirán en sistemas de primer orden con retardo. De esta forma se podrán hacer extensibles a cualquier sistema que pueda aproximarse mediante un equivalente FOPDT (First Order Plus Dead Time) y se hará mas sencillo el análisis al reducir el número de polos.

- En el cuarto capítulo se desarrollará un método para expresar sistemas MIMO controlados por DMC como sistemas de bloques LTI. Este desarrollo permitirá resolver uno de los problemas actuales del control predictivo: la dificultad de análisis teórico del mismo. Una vez desarrollado el método se validará comparando la salida del sistema de bloques obtenido con la proporcionada por el algoritmo «puro» del DMC. Además se demostrará la utilidad del método utilizándolo para comprobar fácilmente que el DMC actúa como un desacoplador perfecto. 
- En el quinto capítulo se usarán los resultados de los dos capítulos anteriores para formular una serie de reglas de sintonía para sistemas MIMO controlados por DMC. Al igual que en el capítulo tres se validará en método poniéndolo a prueba con varios benchmarks extraídos de la literatura y se comparará con otros métodos existentes.

- En el sexto capítulo se analizarán los resultados obtenidos y se describirán las conclusiones. 


\section{Capítulo 2}

\section{Introducción al control predictivo}

\subsection{Introducción}

En este capítulo se va a explicar brevemente la historia del control predictivo y sus algoritmos más populares. Se prestará especial atención al DMC, del que se hará su desarrollo matemático completo. También se expondrá una breve comparación de las aplicaciones de cada algoritmo.

Tal y como se ha indicado en el capítulo 1, el control predictivo surgió a finales de la década de 1970 para responder a necesidades específicas de industria de procesos que no se podía cubrir con el control PID. No tardó en hacerse popular debido a varios motivos:

- Puede usarse con una enorme variedad de procesos, incluyendo aquellos con retardo de transporte, fase no mínima o inestables.

- Puede implementarse con facilidad en casos multivariables.

- Introduce realimentación de forma natural para compensar perturbaciones medibles.

- la ley de control es fácil de implementar.

- El tratamiento de la restricciones es conceptualmente simple y puede ser sistemáticamente incluido durante el diseño del proceso.

- Es muy útil cuando se conocen las referencias futuras.

Sin embargo, a pesar de estas ventajas, como es de esperar, también tiene inconvenientes:

- Requiere mayor coste computacional que otras soluciones (como el PID).

- El resultado final depende de la exactitud del modelo del proceso.

Aparte de los trabajos de Richalet et al [3] y Cutler y Ramaker [5], también se desarrollaron varios métodos desde una línea de trabajo diferente: El control adaptativo. Son de destacar varios trabajos: 
- Control auto ajustable basado en predictor [19]: este método minimiza, para los valores predichos más recientes, el valor esperado de un criterio cuadrático en un horizonte de control.

- Control adaptativo de horizonte extendido [20]: Este método trata de mantener la salida futura (calculada mediante la ecuación diofantina) cercana a la referencia en un período de tiempo posterior al retraso.

- Control auto adaptativo de predicción extendida [21]: Este método propone una señal de control constante empezando desde el momento presente mientras se usa un predictor sub-óptimo en lugar de resolver la ecuación Diofantina.

Sin embargo, entre estos métodos desarrollados en torno a la idea de control adaptativo, el más popular es el control predictivo generalizado (Generalized Predictive Control, GPC,que se describirá más adelante), que se distingue del control predictivo basado en modelos dinámicos en el uso de la ecuación Diofantina para realizar predicciones.

La falta de estabilidad original para controladores de horizonte finito siguió siendo un problema. Esto propició la aparición de métodos que garantizaban estabilidad en los años 90: CRHPC [9] y SIORHC [10]. Kouvaritakis et al [11] presentaron una formulación estable del GPC que estabiliza el proceso antes de minimizar la función objetivo.

La estabilidad de los problemas con restricciones fue un problema serio, ya que al principio el hecho de que el algoritmo resolviera un problema de optimización no garantizaba que el resultado fuera estable. El uso de restricciones, funciones de Lyapunov o conjuntos de invariantes ha permitido la creación de técnicas que garantizan la estabilidad del sistema.

También se han obtenido resultados prometedores en el campo de la robustez. La idea clave es tener en cuenta ciertas incertidumbres del proceso de manera explícita y diseñar el control predictivo para que optimice la función objetivo para la peor situación de incertidumbres.

El control predicitvo basado en modelos se considera una técnica madura para ser usado con procesos lineales y lentos, típicos en la industria de procesos. Durante muchos años se ha considerado que los procesos más complejos (rápidos, híbridos o no lineales) estaban fuera de su campo de aplicación, aunque esta concepción comenzó a cambiar en el momento en el que las aplicaciones del MPC se expandieron a campos cada vez más alejados de la industria de procesos que lo vio nacer, como el control de vehículos terrestres no operados [22],los procesos no lineales [23], la industria aeroespacial [24] [25], el control de robots [26] o la anestesia clínica [27]. 


\subsection{La historia del MPC en la industria}

La historia del control predictivo puede dividirse en cuatro generaciones (como puede verse en la Figura 2.1, [1]), siendo la última de ellas la que ha llegado hasta la actualidad. Los métodos que se describen en este apartado no son los únicos existentes. Hay más métodos de control predictivo, pero muchos de ellos son propiedad de las empresas que los han desarrollado y sus datos no han sido publicados.

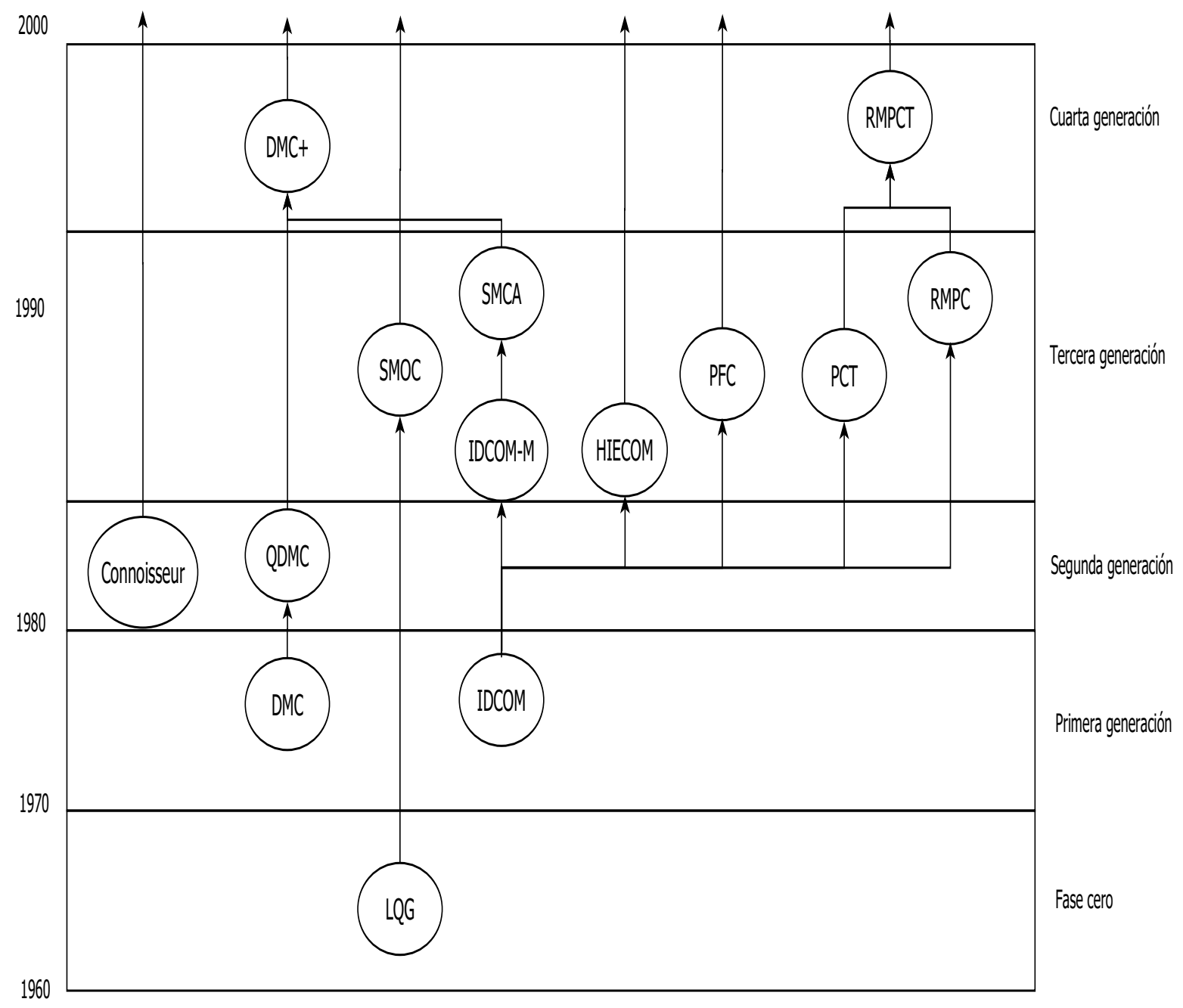

Figura 2.1: Evolución histórica del MPC en la industria

\subsubsection{La fase cero. El regulador LQG.}

La historia del control predictivo comenzó en la década de los sesenta cuando Kalman et al ([28],[29]) diseñaron un regulador lineal cuadrático que minimizaba una función objetivo 
cuadrática sin restricciones. El proceso considerado era el siguiente:

$$
\begin{gathered}
\mathrm{x}_{\mathrm{k}+1}=\mathrm{Ax}_{\mathrm{k}}+\mathrm{Bu}_{\mathrm{k}}+\mathrm{Gw}_{\mathrm{k}} \\
\mathrm{y}_{\mathrm{k}}=\mathrm{cx}_{\mathrm{k}}+\xi_{\mathrm{k}}
\end{gathered}
$$

$\mathrm{El}$ vector u representa las variables manipuladas, el vector y las variables controladas. Las perturbaciones $\left(\xi_{\mathrm{k}}\right)$ y el ruido de medida $\left(\mathrm{w}_{\mathrm{k}}\right)$ se consideran ruido Gaussiano con media cero. La función objetivo a minimizar $(\phi)$ penaliza los valores esperados del cuadrado de las variables manipuladas y las desviaciones del origen e incluye matrices separadas de estado y pesos de entrada (Q y R) para permitir ajustes.

$$
\begin{gathered}
\phi=E(J) \\
J=\sum_{j=1}^{\infty}\left(\left\|\mathrm{x}_{\mathrm{k}+\mathrm{j}}\right\|_{\mathrm{Q}}^{2}+\left\|\mathrm{u}_{\mathrm{k}+\mathrm{j}}\right\|_{\mathrm{R}}^{2}\right)
\end{gathered}
$$

Los términos normalizados de la función objetivo se describen de la siguiente manera:

$$
\|\mathrm{x}\|_{\mathrm{Q}}^{2}=\mathrm{x}^{\mathrm{T}} \mathrm{Qx}
$$

La formulación utilizada asume que las variables se escriben en términos de desviación respecto a un estado deseado. La solución de este problema, conocida como controlador Lineal cuadrático Gaussiano (Linear Quadratic Gaussian, LQG) implica dos pasos:

- La salida medida $\left(\mathrm{y}_{\mathrm{k}}\right)$ se usa para calcular un estado estimado óptimo, $\hat{\mathrm{x}}_{\mathrm{k} \mid \mathrm{k}}$.

$$
\begin{gathered}
\hat{\mathrm{x}}_{\mathrm{k} \mid \mathrm{k}-1}=\mathrm{A} \hat{\mathrm{x}}_{\mathrm{k}-1 \mid \mathrm{k}-1}+\mathrm{Bu}_{\mathrm{k}-1} \\
\hat{\mathrm{x}}_{\mathrm{k} \mid \mathrm{k}}=\hat{\mathrm{x}}_{\mathrm{k} \mid \mathrm{k}-1}+\mathrm{K}_{\mathrm{f}}\left(\mathrm{y}_{\mathrm{k}}-\mathrm{C} \hat{\mathrm{x}}_{\mathrm{k} \mid \mathrm{k}-1}\right)
\end{gathered}
$$

- El valor óptimo de la variable manipulada se calcula usando un controlador de estado proporcional óptimo.

$$
\mathrm{u}_{\mathrm{k}}=-\mathrm{K}_{\mathrm{c}} \hat{\mathrm{x}}_{\mathrm{k} \mid \mathrm{k}}
$$

Debido a su horizonte infinito el controlador LQG presentaba una gran estabilidad. Sus aplicaciones prácticas no se hicieron esperar [30]. A lo largo de los años se han desarrollado miles de aplicaciones y alrededor de 400 patentes [31].

Sin embargo, este avance apenas tuvo impacto en el desarrollo de la teoría de control en la industria de procesos debido a varias razones:

- La teoría del controlador LQG no prevé la existencia de restricciones. En muchas ocasiones el punto óptimo de un controlador está en la intersección de las restricciones [32], manteniendo el sistema cerca de las mismas sin sobrepasarlas.

- Las no linealidades del proceso 
- La dificultad de obtener modelos matemáticos de los procesos a controlar. De hecho una de las áreas donde el LGQ tuvo éxito fue la aeronáutica donde es posible obtener modelos matemáticos. Por contra en la industria química y de procesos donde conseguir estos modelos es complicado, este método apenas tuvo impacto [1].

- Motivos culturales. Los técnicos de instrumentación y los ingenieros de control de aquella época no estaban familiarizados con los conceptos de LQG o no lo veían práctico[1].

\subsubsection{La primera generación. DMC y MAC}

Si bien el controlador LQG no tuvo impacto en la industria de procesos, si que sirvió para que en el seno de la misma se empezaran a desarrollar métodos basados en modelos en los que el problema dinámico de control se resolvía en línea a cada ejecución del control. El siguiente paso en el desarrollo del control predictivo se dio en la década de los setenta, cuando (coincidiendo con el desarrollo de los ordenadores) en el seno de la industria apareció la primera generación de algoritmos de control predictivo ([1]), entre los que cabe destacar el Control Dinámico Matricial (Dynamic Matrix Control, DMC) presentado por Cutler y Ramaker ([33],[5]) y el Control Predictivo Heurístico por Modelo (Model Predictive Heuristic Control, MPHC), presentado por Richalet et al ([3], [4]) y que más tarde se llamaría Control Algorítmico por Modelo (Model Algorithm Control, MAC) o IDCOM (Identification and Command). Estos algoritmos compartían una misma filosofía:

- Se usa un modelo dinámico del proceso para predecir el efecto de las futuras acciones de control en la salida.

- Se calcula para cada instante una secuencia de incrementos de control que minimizan una función objetivo

- Solo se usa el primer incremento de control de la secuencia calculada. Entonces se desplaza el horizonte hacia el futuro y se vuelve a aplicar el algoritmo.

La diferencia entre los distintos métodos consiste en el modelo usado para representar el proceso, la función de coste a minimizar y el hecho de que el MAC incluía el tratamiento de restricciones en su formulación mientras que el DMC se formuló inicialmente sin tratamiento de las mismas.

Esta primera generación de controladores predictivos tuvo un gran impacto en la comunidad de control y definió las pautas a seguir durante los años siguientes.

\subsubsection{La segunda generación. QDMC}

La primera generación definida en el apartado anterior demostró una excelente capacidad para resolver problemas sin restricciones. Pero el tratamiento de las mismas seguía siendo algo «ad hoc». Los ingenieros de Shell Oil detectaron esta debilidad en su algoritmo y se decidieron a subsanarla. Para ello convirtieron el DMC en un problema cuadrático en 
el que las restricciones aparecen explícitamente. Este nuevo método (denominado QDMC) fue descrito inicialmente por Cutler et al [34] y García y Morshedi [35]. García y Mosherdi demostraron que la función objetivo del DMC puede reescribirse como un problema cuadrático. A pesar de que el QDMC puede considerarse un algoritmo complejo, el problema cuadrático es una de los problemas de optimización más sencillos. El hessiano del problema cuadrático es positivo para plantas lineales por lo que el problema es convergente y puede resolverse mediante códigos de optimización normalizados y comerciales.

García y Mosherdi aportaron con su investigación resultados del control de la presión de vapor ajustando la presión de gas natural en tres quemadores además de numerosas aplicaciones de hasta [12x12]. Destacaron que por encima de todo que el QDMC era particularmente adecuado para la optimización on line, donde proporcionaba una transición suave de un punto de operación con restricciones a otro.

Puede resumirse que el QDMC fue el representante de una generación de controladores en los que formuló el control predictivo como un problema cuadrático que se podía resolver mediante códigos normalizados.

\subsubsection{La tercera generación}

Los problemas resueltos con la segunda generación de controladores permitieron una mayor aceptación y avance del control predictivo en la industria. Sin embargo, estos nuevos avances llevaron a nuevos problemas que el QDMC y sus contemporáneos no podían solucionar, la factibilidad. En la práctica pueden perderse temporalmente entradas y salidas de proceso (debido, por ejemplo, a fallos en los sensores, saturación de válvulas y operación directa) y después volver a recuperarlas. Esto significa que la estructura del problema y los grados de libertad cambian dinámicamente, dando lugar a tres situaciones distintas:

- Planta cuadrada: El número de variable manipuladas (VM) y de variables controladas (VC) es el mismo. La solución de control es única. Este caso es raro en la práctica.

- Planta grande: VM es mayor que VC. Los grados de libertad adicionales se usan para cumplir objetivos de control adicionales, como llevar la planta a un punto óptimo.

- Planta pequeña: VM es menor que VC. En este caso es imposible cumplir todos los objetivos de control y deben relajarse de alguna forma, como, por ejemplo, minimizando la violación de restricciones mediante un problema cuadrático.

Otro problema que surgió durante estos años fue la tolerancia a los fallos. Un controlador predictivo no debía desconectarse cuando perdía una señal, sino permanecer activo e intentar controlar la «sub planta» resultante. 
Y por último estaba la dificultad de traducir los requisitos de control en pesos relativos en una única función objetivo. Esta función debía incluir pesos para violaciones de la referencia, violación de restricciones, acciones de control y desvío de la solución óptima. Tener todas estas variables en una única función objetivo es complicado y, a veces, imposible. Es fácil que intentando cumplir un objetivo se incumplan los demás. E incluso cuando es posible debe prestarse especial atención de evitar problemas de escalado que lleven a problemas mal condicionados. Sobre este problema Prett y García [36] opinaron que la combinación de varios objetivos en una única función objetivo no permite al diseñador reflejar los verdaderos requisitos para las prestaciones.

Los problemas anteriores llevaron a los ingenieros de Adersa, Setpoint y Shell (Francia) a desarrollar nuevos algoritmos predictivos que los solucionaran:

- IDCOM-M: Desarrollado por Setpoint incluía dos funciones objetivo: Una para las salidas y, en caso de existir grados de libertad extra, otra para las entradas. Este método fue continuamente desarrollado por Setpoint hasta fundirlo con el resto de sus productos de identificación simulación y control creando un único producto, Setpoint Multivariable Control Architecture (SMCA).

- Shell Multivariable Optimizing Controller (SMOC): Desarrollado por Shell en Francia y descrito como el punto intermedio entre los controladores predictivos y los espacios de estado.

- PTC desarrollado por Profimatics

- RMPC desarrollado por Honeywell

En concreto, SMOC introdujo algunas características que actualmente se consideran fundamentales en un controlador predictivo moderno:

- Se usa un modelo en espacio de estado. De esta forma se puede representar todo el rango de la dinámica lineal (estabilidad, inestabilidad e integración).

- Presenta un modelo explícito para perturbaciones para describir las perturbaciones no medidas.

- Usa un filtro de Kalman para estimar los estados de la planta y perturbaciones no medidas de la salida medida.

- Distingue entre las variables controladas que aparecen en el objetivo de control y las variables de alimentación que se usan para la estimación de estado.

- Tratamiento de restricciones mediante un problema cuadrático.

El algoritmo SMOC es similar a resolver el LQR con restricciones de entrada y salida, pero con un horizonte finito (y, por lo tanto, sin la potente capacidad estabilizadora del LQR). 


\subsubsection{La cuarta generación.}

A finales de la década de los noventa, la competencia y las fusiones entre desarrolladores de soluciones MPC cambiaron el panorama del mercado.

- Honeywell adquirió Profimatics, formando Honeywell Hi-Spec Solutions. Los algoritmos de ambas empresas se fusionaron para crear RMPCT.

- Aspen Tech adquirió Setpoint Inc, DMC Corporation y Treiber Controls. Los productos DMC y SMCA se unieron en un solo paquete: DMC-Plus.

En la actualidad, esta cuarta generación sigue vendiéndose hoy en día con varias mejoras como interfaces gráficas en Windows, múltiples niveles de optimización para atender a objetivos prioritarios y tecnología de identificación mejorada basada en predicción de errores.

\subsection{Estrategia del MPC}

A pesar de las diferencias existentes entre los diferentes métodos de MPC todos responden a la misma estrategia, representada en la Figura 2.2:

1. Los valores de salida para un determinado horizonte Pr, llamado horizonte de predicción, se predicen para cada instante t. Estos valores $\hat{y}(t+k \mid t)$ dependen de los valores conocidos hasta el instante $t$ (entradas y salidas pasadas) y de las futuras señales de control $\mathrm{u}(\mathrm{t}+\mathrm{k} \mid \mathrm{t}), \mathrm{k}=0 \ldots \mathrm{N}-1$, que se enviarán al sistema y se calcularán.

2. El conjunto de futuras señales de control se calcula optimizando un determinado criterio para mantener al sistema lo más cerca posible de la referencia deseada $\mathrm{w}(\mathrm{t}+$ k). Este criterio suele ser una función cuadrática del error entre la salida predicha y la referencia. El esfuerzo de control se incluye en la función objetivo en la mayoría de casos.

3. La señal de control en el instante actual, $u(t \mid t)$, se manda a la planta mientras que las señales para los siguientes instantes se descartan, ya que el siguiente instante de muestreo, $\mathrm{y}(\mathrm{t}+1)$, ya se conoce y se repetirá el paso 1 con este nuevo valor y las secuencias se actualizan. Eso significa que el valor de $u(t+1 \mid t+1)$ es diferente a $\mathrm{u}(\mathrm{t}+1 \mid \mathrm{t})$ al haberse actualizado la secuencia con nueva información.

La estructura básica necesaria para llevar a cabo esta estrategia se representa en la Figura 2.3. Se usa un modelo para predecir las futuras salidas de la planta, basándose en valores actuales y pasados y en las acciones de control calculadas. Estas acciones son calculadas por el optimizador usando la función de coste y las restricciones.

Como puede verse, el modelo del proceso tiene un papel decisivo en el controlador. Debe representar adecuadamente la dinámica del proceso para poder predecir las salidas futuras, pero a la vez debe ser fácil de entender e implementar. De hecho, el tipo modelo 


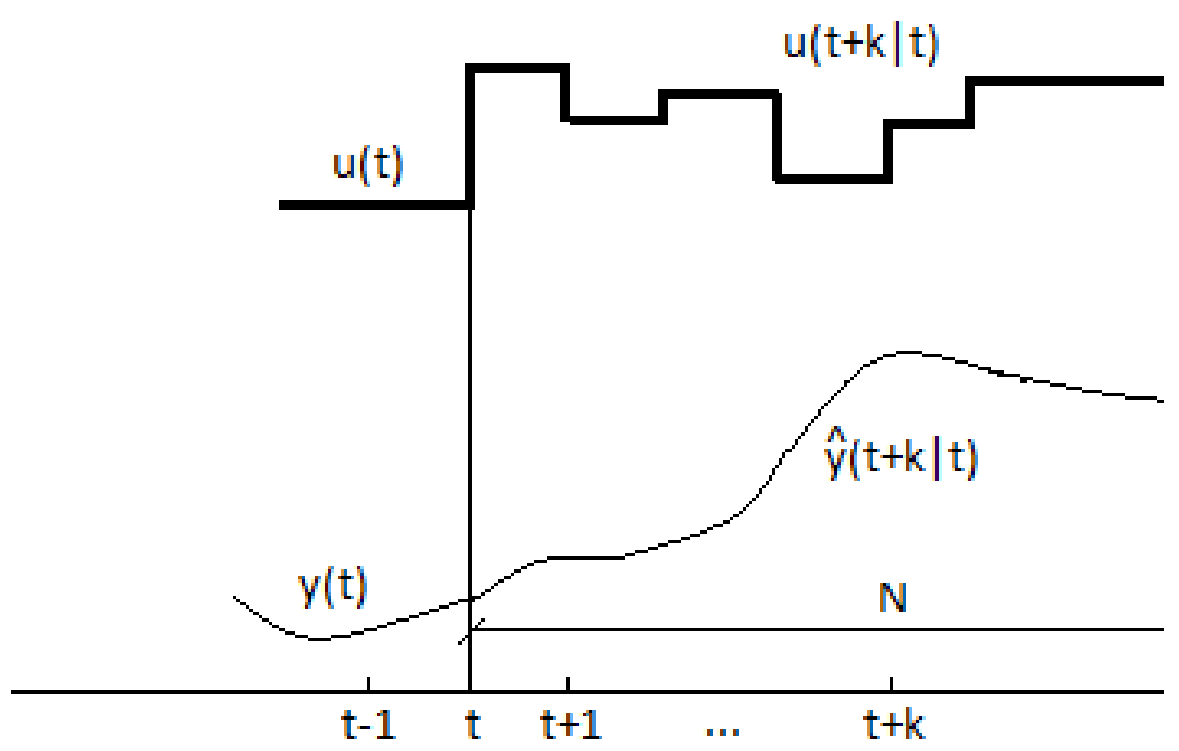

Figura 2.2: Estrategia MPC

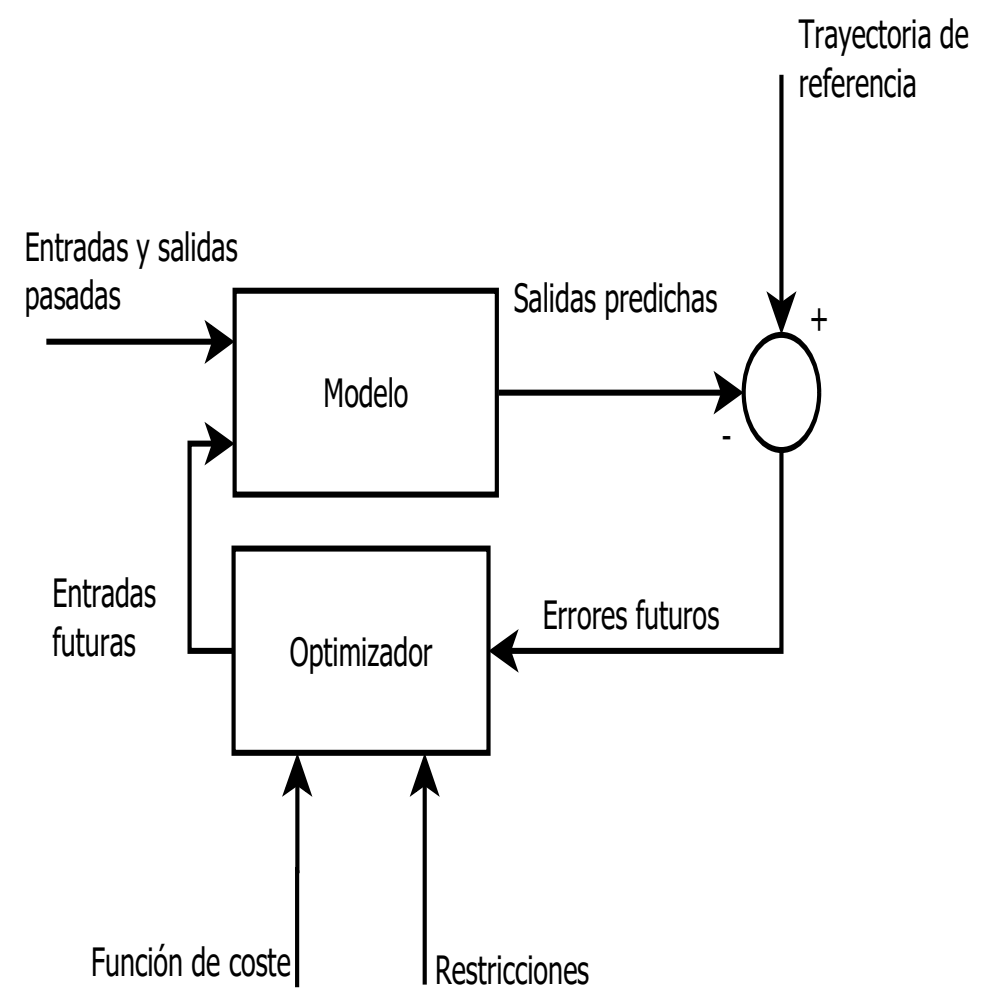

Figura 2.3: Estructura básica MPC 
utilizado es una de las diferencias fundamentales de los distintos tipos de MPC, junto con la función de coste.

Esta estrategia de control es similar a la usada para conducir un coche. El conductor conoce la trayectoria para un horizonte de control finito y teniendo en cuanta las características del vehículo (modelo) y los valores pasados (espejos retrovisores), decide que acciones de control son las más adecuadas (volante, frenos y acelerador). Aplicando esta analogía a un PID, se puede deducir que dicho controlador equivaldría a conducir usando únicamente el espejo retrovisor (realimentación de error).

\subsection{Métodos de control predictivo}

Tal y como se ha mencionado anteriormente, la diferencia entre los distintos métodos de control predictivo son principalmente el modelo usado para predecir el comportamiento del proceso y la función de coste a optimizar.

En el entorno industrial se prefieren los modelos obtenidos midiendo directamente de la planta, como los modelos de respuesta impulso y/o escalón. Estos modelos se obtienen introduciendo como entrada un impulso o un escalón en la planta y midiendo su salida. Son sencillos de obtener, intuitivos y pueden usarse en plantas multivariable. Sin embargo, necesitan una gran cantidad de parámetros y solo son válidos para procesos estables en lazo abierto. DMC y MAC son un ejemplo de controladores predictivos que usan este tipo de modelo.

En el entorno académico se prefieren modelos de espacios de estado o funciones de transferencia. El modelo de espacios de estado permite un tratamiento sencillo de la robustez y la estabilidad.

En un punto intermedio entre los modelos prácticos y académicos está la función de transferencia. La derivación de este modelo es más complicada, pero requiere menos parámetros.Además, el retraso (algo frecuente en la industria) puede tratarse de forma más sencilla que con los espacios de estado. Un ejemplo de controlador predictivo que usa este modelo, es GPC.

Los algoritmos más conocidos se presentan a continuación.

\section{Control Dinámico Matricial-DMC}

El DMC usa como modelo la respuesta a escalón unitario del proceso. El proceso debe ser estable y sin integradores.

$$
y(t)=\sum_{i=1}^{\infty} g_{i} \Delta u(t-i)
$$


Donde $g_{\mathrm{i}}$ son los coeficientes de respuesta a escalón unidad, $\Delta \mathrm{u}$ es el incremento de control, y es la respuesta del sistema y $\mathrm{n}(\mathrm{t})$ son las perturbaciones. Así que los valores predecidos serán (Empezando las predicciones en el instante $\mathrm{t}$ ):

$\hat{\mathrm{y}}(\mathrm{t}+\mathrm{k})=\sum_{\mathrm{i}=1}^{\infty} \mathrm{g}_{\mathrm{i}} \Delta \mathrm{u}(\mathrm{t}+\mathrm{k}-\mathrm{i})+\hat{\mathrm{n}}(\mathrm{t}+\mathrm{k})=\sum_{\mathrm{i}=1}^{\mathrm{k}} \mathrm{g}_{\mathrm{i}} \Delta \mathrm{u}(\mathrm{t}+\mathrm{k}-\mathrm{i})+\sum_{\mathrm{i}=\mathrm{k}+1}^{\infty} \mathrm{g}_{\mathrm{i}} \Delta \mathrm{u}(\mathrm{t}+\mathrm{k}-\mathrm{i})+\hat{\mathrm{n}}(\mathrm{t}+\mathrm{k})$

Considerado perturbaciones constantes (

$$
\hat{\mathrm{n}}(\mathrm{t}+\mathrm{k})=\hat{\mathrm{n}}(\mathrm{t})=\mathrm{y}_{\mathrm{m}}(\mathrm{t})-\hat{\mathrm{y}}(\mathrm{t})=\mathrm{y}_{\mathrm{m}}(\mathrm{t})-\sum_{\mathrm{i}=1}^{\infty} \mathrm{g}_{\mathrm{i}} \Delta \mathrm{u}(\mathrm{t}-\mathrm{i})
$$

Entonces, la ecuación 2.10 puede escribirse:

$\hat{\mathrm{y}}(\mathrm{t}+\mathrm{k})=\sum_{\mathrm{i}=1}^{\mathrm{k}} \mathrm{g}_{\mathrm{i}} \Delta \mathrm{u}(\mathrm{t}+\mathrm{k}-\mathrm{i})+\sum_{\mathrm{i}=\mathrm{k}+1}^{\infty} \mathrm{g}_{\mathrm{i}} \Delta \mathrm{u}(\mathrm{t}+\mathrm{k}-\mathrm{i})+\mathrm{y}_{\mathrm{m}}(\mathrm{t})-\sum_{\mathrm{i}=1}^{\infty} \mathrm{g}_{\mathrm{i}} \Delta \mathrm{u}(\mathrm{t}-\mathrm{i})=\sum_{\mathrm{i}=1}^{\mathrm{k}} \mathrm{g}_{\mathrm{i}} \Delta \mathrm{u}(\mathrm{t}+\mathrm{k}-\mathrm{i})+\mathrm{f}(\mathrm{t}+\mathrm{k})$

Siendo $\mathrm{f}(\mathrm{t}+\mathrm{k})$ la respuesta libre, la parte de la respuesta que no depende de las acciones de control futuras, descrita como sigue:

$$
\mathrm{f}(\mathrm{t}+\mathrm{k})=\mathrm{y}_{\mathrm{m}}(\mathrm{t})+\sum_{\mathrm{i}=1}^{\infty}\left(\mathrm{g}_{\mathrm{k}+\mathrm{i}}-\mathrm{g}_{\mathrm{i}}\right) \Delta \mathrm{u}(\mathrm{t}-\mathrm{i})
$$

Si el proceso es asintóticamente estable, los coeficientes de la respuesta a escalón, $\mathrm{g}_{\mathrm{i}}$, tenderán a ser constantes tras $\mathrm{N}$ períodos de muestreo. Por lo que:

$$
\left(\mathrm{g}_{\mathrm{k}+\mathrm{i}}-\mathrm{g}_{\mathrm{i}}\right) \rightarrow 0, \mathrm{i}>\mathrm{N}
$$

Y la ecuación 2.13, puede simplificarse:

$$
\mathrm{f}(\mathrm{t}+\mathrm{k})=\mathrm{y}_{\mathrm{m}}(\mathrm{t})+\sum_{\mathrm{i}=1}^{\mathrm{N}}\left(\mathrm{g}_{\mathrm{k}+\mathrm{i}}-\mathrm{g}_{\mathrm{i}}\right) \Delta \mathrm{u}(\mathrm{t}-\mathrm{i})
$$

Aplicando las ecuaciones anteriores para un horizonte de predicción igual a Pr y un horizonte de control igual a M:

$$
\begin{gathered}
\hat{\mathrm{y}}(\mathrm{t}+1 / \mathrm{t})=\mathrm{g}_{1} \Delta \mathrm{u}(\mathrm{t})+\mathrm{f}(\mathrm{t}+1) \\
\hat{\mathrm{y}}(\mathrm{t}+2 / \mathrm{t})=\mathrm{g}_{2} \Delta \mathrm{u}(\mathrm{t})+\mathrm{g}_{1} \Delta \mathrm{u}(\mathrm{t}+1)+\mathrm{f}(\mathrm{t}+1)
\end{gathered}
$$




$$
\hat{\mathrm{y}}(\mathrm{t}+\operatorname{Pr} / \mathrm{t})=\sum_{\mathrm{i}=\operatorname{Pr}-\mathrm{M}+1}^{\operatorname{Pr}} \mathrm{g}_{\mathrm{i}} \Delta \mathrm{u}(\mathrm{t}+\mathrm{Pr}-\mathrm{i})+\mathrm{f}(\mathrm{t}+\mathrm{Pr})
$$

Definiendo la matriz dinámica del sistema como:

$$
\mathbf{G}=\left(\begin{array}{lllr}
\mathrm{g}_{1} & 0 & \ldots & 0 \\
\mathrm{~g}_{2} & \mathrm{~g}_{1} & \ldots & 0 \\
\mathrm{~g}_{\mathrm{M}} & \mathrm{g}_{\mathrm{M}-1} & \ldots & \mathrm{g}_{1} \\
: & : & \ldots & : \\
\mathrm{g}_{\mathrm{Pr}} & \mathrm{g}_{\mathrm{Pr}-1} & \cdots & \mathrm{g}_{\mathrm{Pr}-\mathrm{M}+1}
\end{array}\right)
$$

Usando formulación matricial, se puede escribir:

$$
\hat{\mathbf{y}}=\mathbf{G} \boldsymbol{\Delta} \mathbf{u}+\mathbf{f}
$$

Siendo $\hat{\mathbf{y}}$ un vector de dimensión Pr que contiene las predicciones futuras del sistema en un horizonte de predicción $\operatorname{Pr}, \boldsymbol{\Delta} \mathbf{u}$ un vector de dimensión M que contiene los incrementos de control y $\mathbf{f}$ el vector de respuesta libre. Esta expresión relaciona las salidas futuras con los incrementos de control y se usa para calcular las acciones necesarias para obtener un comportamiento específico.

El objetivo del DMC es encontrar un incremento de control que minimice una determinada función de coste que incluye errores y esfuerzos de control.

$$
\begin{gathered}
\mathrm{J}=\sum_{\mathrm{j}=1}^{\mathrm{p}}(\hat{\mathrm{y}}(\mathrm{t}+\mathrm{j} \mid \mathrm{t})-\mathrm{w}(\mathrm{t}+\mathrm{j}))^{2}+\sum_{\mathrm{j}=1}^{\mathrm{m}} \lambda(\Delta \mathrm{u}(\mathrm{t}+\mathrm{j}-1))^{2} \\
\mathbf{J}=\mathbf{e e}^{\mathbf{T}}+\lambda \boldsymbol{\Delta} \mathbf{u} \boldsymbol{\Delta} \mathbf{u}^{\mathbf{T}}
\end{gathered}
$$

Donde e es el vector de errores y $\boldsymbol{\Delta} \mathbf{u}$ el vector de esfuerzos de control. En un problema sin restricciones, el vector de esfuerzos de control optimizados se obtiene resolviendo la Ecuación 2.23:

$$
\frac{\mathrm{d} \mathbf{J}}{\mathrm{d} \Delta \mathbf{u}}=0
$$

Para resolver la ecuación 2.23 la dividiremos en dos términos: El término de errores $\left(\mathrm{J}_{1}\right)$ y el término de incrementos de control $\left(\mathrm{J}_{2}\right)$.

$$
\begin{gathered}
\mathrm{J}_{1}=\sum_{\mathrm{j}=1}^{\mathrm{p}}(\hat{\mathrm{y}}(\mathrm{t}+\mathrm{j} \mid \mathrm{t})-\mathrm{w}(\mathrm{t}+\mathrm{j}))^{2} \\
\mathrm{~J}_{2}=\sum_{\mathrm{j}=1}^{\mathrm{m}} \lambda(\Delta \mathrm{u}(\mathrm{t}+\mathrm{j}-1))^{2}
\end{gathered}
$$

Teniendo en cuenta que $\hat{y}(t+j \mid t)=\sum_{i=1}^{j} g_{i} \Delta u(t+j-i)+f(t+j)$, se puede expresar $\left(\mathrm{J}_{1}\right)$ de la siguiente manera: 


$$
\begin{aligned}
& J_{1}=\sum_{j=1}^{p}(\hat{y}(t+j \mid t)-w(t+j))^{2}=\sum_{j=1}^{p}\left(\sum_{i=1}^{j} g_{i} \Delta u(t+j-i)+f(t+j)-w(t+j)\right)^{2}= \\
& \sum_{j=1}^{p}\left(\left(\sum_{i=1}^{j} g_{i} \Delta u(t+j-i)\right)^{2}+2 f(t+j) \sum_{i=1}^{j} g_{i} \Delta u(t+j-i)-2 w(t+j) \sum_{i=1}^{j} g_{i} \Delta u(t+j-i)-\right. \\
& \left.2 f(t+j) w(t+j)+f(t+j)^{2}+w(t+j)^{2}\right)
\end{aligned}
$$

Para simplificar el análisis, $\mathrm{J}_{1}$ puede expresarse a su vez como la suma de otros términos que habrá que tratar por separado.

$$
\begin{aligned}
& \mathrm{J}_{11}=\sum_{\mathrm{j}=1}^{\mathrm{p}}\left(\frac{1}{2} \sum_{\mathrm{i}=1}^{\mathrm{j}} \mathrm{g}_{\mathrm{i}} \Delta \mathrm{u}(\mathrm{t}+\mathrm{j}-\mathrm{i})\right)^{2}=\frac{1}{2}\left(\mathrm{~g}_{1} \Delta \mathrm{u}(\mathrm{t})\right)^{2}+\frac{1}{2}\left(\mathrm{~g}_{1} \Delta \mathrm{u}(\mathrm{t}+1)+\mathrm{g}_{2} \Delta \mathrm{u}(\mathrm{t})\right)^{2}+\ldots+ \\
& \frac{1}{2}\left(\mathrm{~g}_{1} \Delta \mathrm{u}(\mathrm{t}+\mathrm{p}-1)+. .+\mathrm{g}_{\mathrm{p}} \Delta \mathrm{u}(\mathrm{t})\right)^{2} \\
& \mathrm{~J}_{12}=\sum_{\mathrm{j}=1}^{\mathrm{p}}\left(\mathrm{f}(\mathrm{t}+\mathrm{j}) \sum_{\mathrm{i}=1}^{\mathrm{j}} \mathrm{g}_{\mathrm{i}} \Delta \mathrm{u}(\mathrm{t}+\mathrm{j}-\mathrm{i})\right)=\left(\mathrm{f}(\mathrm{t}+1) \mathrm{g}_{1} \Delta \mathrm{u}(\mathrm{t})\right)+ \\
& \quad+\left(\mathrm{f}(\mathrm{t}+2)\left(\mathrm{g}_{1} \Delta \mathrm{u}(\mathrm{t}+1)+\mathrm{g}_{2} \Delta \mathrm{u}(\mathrm{t})\right)\right)+\ldots+ \\
& \quad+\left(\mathrm{f}(\mathrm{t}+\mathrm{p})\left(\mathrm{g}_{1} \Delta \mathrm{u}(\mathrm{t}+\mathrm{p}-1)+\ldots+\mathrm{g}_{\mathrm{p}} \Delta \mathrm{u}(\mathrm{t})\right)\right) \\
& \mathrm{J}_{13}=\sum_{\mathrm{j}=1}^{\mathrm{p}}\left(\mathrm{w}(\mathrm{t}+\mathrm{j}) \sum_{\mathrm{i}=1}^{\mathrm{j}} \mathrm{g}_{\mathrm{i}} \Delta \mathrm{u}(\mathrm{t}+\mathrm{j}-\mathrm{i})\right)=\left(\mathrm{w}(\mathrm{t}+1) \mathrm{g}_{1} \Delta \mathrm{u}(\mathrm{t})\right)+\left(\mathrm{w}(\mathrm{t}+2)\left(\mathrm{g}_{1} \Delta \mathrm{u}(\mathrm{t}+1)+\mathrm{g}_{2} \Delta \mathrm{u}(\mathrm{t})\right)\right)+\ldots+ \\
& +\left(\mathrm{w}(\mathrm{t}+\mathrm{p})\left(\mathrm{g}_{1} \Delta \mathrm{u}(\mathrm{t}+\mathrm{p}-1)+\ldots+\mathrm{g}_{\mathrm{p}} \Delta \mathrm{u}(\mathrm{t})\right)\right) \\
& \mathrm{J}_{2}=\lambda \sum_{\mathrm{j}=1}^{\mathrm{p}}(\Delta \mathrm{u}(\mathrm{t}+\mathrm{j}-1))^{2}=\lambda \Delta \mathrm{u}(\mathrm{t})^{2}+\lambda \Delta \mathrm{u}(\mathrm{t}+1)^{2}+\ldots+\lambda \Delta \mathrm{u}(\mathrm{t}+\mathrm{m}-1)^{2}
\end{aligned}
$$

Teniendo en cuenta todo lo anterior, se pueden calcular las derivadas parciales de $\mathrm{J}$ respecto a los incrementos de control.

$$
J=\sum_{j=1}^{p}\left(J_{11}+J_{12}-J_{13}-2 f(t+j) w(t+j)+f(t+j)^{2}+w(t+j)^{2}\right)+J_{2}
$$




$$
\begin{gathered}
\frac{\delta \mathrm{J}}{\delta \Delta \mathrm{u}(\mathrm{t})}=2\left(\frac{\delta \mathrm{J}_{11}}{\delta \Delta \mathrm{u}(\mathrm{t})}+\frac{\delta \mathrm{J}_{12}}{\delta \Delta \mathrm{u}(\mathrm{t})}-\frac{\delta \mathrm{J}_{13}}{\delta \Delta \mathrm{u}(\mathrm{t})}\right)+2 \Delta \mathrm{u}(\mathrm{t})=0 \\
\frac{\delta \mathrm{J}}{\delta \Delta \mathrm{u}(\mathrm{t}+1)}=2\left(\frac{\delta \mathrm{J}_{11}}{\delta \Delta \mathrm{u}(\mathrm{t}+1)}+\frac{\delta \mathrm{J}_{12}}{\delta \Delta \mathrm{u}(\mathrm{t}+1)}-\frac{\delta \mathrm{J}_{13}}{\delta \Delta \mathrm{u}(\mathrm{t}+1)}\right)+2 \Delta \mathrm{u}(\mathrm{t}+1)=0
\end{gathered}
$$

$\frac{\delta \mathrm{J}}{\delta \Delta \mathrm{u}(\mathrm{t}+\mathrm{m}-1)}=2\left(\frac{\delta \mathrm{J}_{11}}{\delta \Delta \mathrm{u}(\mathrm{t}+\mathrm{m}-1)}+\frac{\delta \mathrm{J}_{12}}{\delta \Delta \mathrm{u}(\mathrm{t}+\mathrm{m}-1)}-\frac{\delta \mathrm{J}_{13}}{\delta \Delta \mathrm{u}(\mathrm{t}+\mathrm{m}-1)}\right)+2 \Delta \mathrm{u}(\mathrm{t}+\mathrm{m}-1)=0$

Formándose un sistema de m ecuaciones con m incógnitas, los incrementos de control. Si se tienen en cuenta las siguientes igualdades:

$$
\begin{aligned}
& \left(\begin{array}{c}
\frac{\delta \mathrm{J}_{11}}{\delta \Delta \mathrm{u}_{\mathrm{t}}} \\
\frac{\delta \mathrm{J}_{11}}{\delta \Delta \mathrm{u}(\mathrm{t}+1)} \\
\cdot \\
\cdot \\
\frac{\delta \mathrm{J}_{11}}{\delta \Delta \mathrm{u}(\mathrm{t}+\mathrm{m}-1)}
\end{array}\right)=\mathbf{G}^{\mathbf{T}} \mathbf{G}\left(\begin{array}{c}
\Delta \mathrm{u}(\mathrm{t}) \\
\Delta \mathrm{u}(\mathrm{t}+1) \\
\cdot \\
\cdot \\
\cdot \\
\Delta \mathrm{u}(\mathrm{t}+\mathrm{m}-1)
\end{array}\right)=0 \\
& \left(\begin{array}{c}
\frac{\delta \mathrm{J}_{12}}{\delta \Delta \mathrm{u}(\mathrm{t})} \\
\frac{\delta \mathrm{J}_{12}}{\delta \Delta \mathrm{u}(\mathrm{t}+1)} \\
\cdot \\
\cdot \\
\frac{\delta \mathrm{J}_{12}}{\delta \Delta \mathrm{u}(\mathrm{t}+\mathrm{m}-1)}
\end{array}\right)=\mathbf{G}^{\mathbf{T}}\left(\begin{array}{c}
\mathrm{f}(\mathrm{t}+1) \\
\mathrm{f}(\mathrm{t}+2) \\
\cdot \\
\cdot \\
\cdot \\
\mathrm{f}(\mathrm{t}+\mathrm{p})
\end{array}\right)=0 \\
& \left(\begin{array}{c}
\frac{\delta \mathrm{J}_{13}}{\delta \Delta \mathrm{u}(\mathrm{t})} \\
\frac{\delta \mathrm{J}_{13}}{\delta \Delta \mathrm{u}(\mathrm{t}+1)} \\
\cdot \\
\cdot \\
\frac{\delta \mathrm{J}_{13}}{\delta \Delta \mathrm{u}(\mathrm{t}+\mathrm{m}-1)}
\end{array}\right)=\mathbf{G}^{\mathbf{T}}\left(\begin{array}{c}
\mathrm{w}(\mathrm{t}+1) \\
\mathrm{w}(\mathrm{t}+2) \\
\cdot \\
\cdot \\
\cdot \\
\mathrm{w}(\mathrm{t}+\mathrm{p})
\end{array}\right)=0
\end{aligned}
$$

El sistema de ecuaciones puede expresarse de la siguiente manera:

$$
\left(\mathbf{G}^{\mathbf{T}} \mathbf{G}+\lambda \mathbf{I}\right) \Delta \mathbf{u}+\mathbf{G}^{\mathbf{T}}(\mathbf{f}-\mathbf{w})=0
$$




$$
\left(\mathbf{G}^{\mathbf{T}} \mathbf{G}+\lambda \mathbf{I}\right) \Delta \mathbf{u}=\mathbf{G}^{\mathbf{T}}(\mathbf{w}-\mathbf{f})
$$

Siendo el resultado:

$$
\Delta \mathbf{u}=\left(\mathbf{G}^{\mathbf{T}} \mathbf{G}+\lambda \mathbf{I}\right)^{-\mathbf{1}} \mathbf{G}^{\mathbf{T}}(\mathbf{w}-\mathbf{f})
$$

Un ejemplo de producto industrial que usa DMC es «AspenTech». Existen alrededor de 1800 aplicaciones conocidas de «AspenTech», mayoría de las cuales pertenecen al campo de la refinería.

\section{Control Algorítmico por Modelo}

Este método es muy similar al DMC salvo por las siguientes diferencias:

- Usa un modelo de respuesta a impulso que solo es válido para procesos estables. Calcula el valor de $\mathrm{u}(\mathrm{t})$ en lugar de $\Delta \mathrm{u}(\mathrm{t})$.

- No utiliza el concepto de horizonte de control, por lo que se calculan tantas señales de control como futuras salidas.

- Introduce una trayectoria de referencia que evoluciona desde la salida real hasta la referencia deseada de acuerdo a una constante de tiempo.

- La función de coste a minimizar es la varianza del error entre la trayectoria de referencia y el error.

\section{Control Predictivo funcional}

Este método fue desarrollado para procesos rápidos. Utiliza un modelo de «estado en espacio» (state space model) del proceso y puede trabajar con modelos internos inestables lineales y no lineales. La dinámica no lineal puede introducirse en la forma de un modelo de estado en espacio no lineal.

El PFC tiene dos características distintivas:

- Puntos de coincidencia: Este concepto se usa para simplificar el cálculo considerando solo un subconjunto de puntos en el horizonte de predicción. Las salidas futuras predichas y deseadas deben coincidir en esos puntos, no en todo el horizonte de predicción.

- El controlador parametriza la señal de control usando un conjunto de funciones de base polinómicas. Esto permite especificar perfiles de entrada relativamente complejos a lo largo de un gran horizonte usando un número pequeño de parámetros. Escoger la familia de estas funciones de base establece muchas de las propiedades del perfil de entrada computado. Se pueden seleccionar estas funciones para que sigan una referencia polinómica sin retraso. 
La función de coste a minimizar es:

$$
J=\sum_{j=1}^{n_{H}}\left[\hat{y}\left(t+h_{j}\right)-w\left(t+h_{j}\right)\right]^{2}
$$

Un ejemplo de producto industrial que usa PFC es «Adersa».

\section{Control Predictivo Generalizado}

Las predicciones del GPC se basan en un modelo CARIMA:

$$
\mathrm{A}\left(\mathrm{z}^{-1}\right) \mathrm{y}(\mathrm{t})=\mathrm{B}\left(\mathrm{z}^{-1}\right) \mathrm{z}^{-\mathrm{d}} \mathrm{u}(\mathrm{t}-1)+\mathrm{C}\left(\mathrm{z}^{-1}\right) \frac{\mathrm{e}(\mathrm{t})}{\Delta}
$$

Donde las perturbaciones no medibles vienen dadas por un ruido blanco coloreado por $\mathrm{C}\left(\mathrm{z}^{-1}\right)$. Como su verdadero valor es difícil de conocer, este polinomio puede usarse para un óptimo rechazo de perturbaciones, aunque su papel en la mejora de la robustez es más convincente.

La derivación de la predicción óptima se hace resolviendo una ecuación Diofantina cuya solución puede obtenerse mediante un algoritmo recursivo eficiente.

La función de coste a minimizar es la siguiente:

$$
J\left(N_{1}, N_{2}, N_{u}\right)=\sum_{j=N_{1}}^{N_{2}} \delta(j)[\hat{y}(t+j \mid t)-w(t+j)]^{2}+\sum_{j=N_{1}}^{N_{2}} \lambda(j)[\Delta u(t+j-1)]^{2}
$$

Las secuencias de peso $\delta(\mathrm{j})$ y $\Delta \mathrm{u}$ se suelen seleccionar constantes o exponencialmente crecientes y la trayectoria de referencia $\mathrm{w}(\mathrm{t}+\mathrm{j})$ puede generarse por una simple recursión que empieza en la salida actual y tiene exponencialmente a la referencia deseada.

\subsubsection{Tecnologías Industriales}

Las siguientes empresas (y sus productos) se pueden considerar como representativas del estado del arte del control predictivo en la industria:

- Aspentech: DMC-plus

- Adersa: Identification and Command (IDCOM), Hierarchical Constraint Control (HIECON) y PFC.

- Honeywell Profomatics: Robust Model Predictive Technology (RMPCT) y Predictive Control Technology (PCT)

- Shell global solutions: Shell multivariable optimizing control (SMOC)

- Schneider electrics: Control and identification package (Connoisseur) 
Cuadro 2.1: Productos representativos de control predictivo

\begin{tabular}{|c|c|c|}
\hline Empresa & Producto MPC lineal & Producto MPC no lineal \\
\hline Aspentech & DMC-plus & Aspen target \\
\hline Adersa & $\begin{array}{c}\text { Hierarchical Constraint Control (HIECON) } \\
\text { Identification and Command (IDCOM) } \\
\text { PFC }\end{array}$ & PFC \\
\hline Honeywell Profomatics & $\begin{array}{c}\text { Robust Model Predictive Technology (RMPCT) } \\
\text { Predictive Control Technology (PCT) }\end{array}$ & \\
\hline Shell global solutions & Shell multivariable optimizing control (SMOC) & \\
\hline Schneider electrics & Control and identification package (Connoisseur) & MVC \\
\hline Continetal Controls & & NOVA-NLC \\
\hline DOT products & & Process Perfecter \\
\hline Pavilion Tecnologies & & \\
\hline
\end{tabular}

Cuadro 2.2: Aplicaciones industriales del control predictivo

\begin{tabular}{|c|c|c|c|c|c|}
\hline Área & Aspentech & Honeywell & Adersa & Setpoint Inc & Treiber \\
\hline Refinería & 1200 & 480 & 280 & 320 & 250 \\
\hline Petroquímica & 450 & 80 & 0 & 40 & 0 \\
\hline Química & 100 & 20 & 3 & 20 & 150 \\
\hline Pulpa y papel & 18 & 50 & 0 & 0 & 5 \\
\hline Gas & 0 & 10 & 0 & 0 & 0 \\
\hline Utilidades & 0 & 10 & 10 & 0 & 0 \\
\hline Separación de Aire & 0 & 0 & 0 & 0 & 5 \\
\hline Minería y metalúrgica & 8 & 6 & 7 & 2 & 6 \\
\hline Procesado de alimentos & 0 & 0 & 41 & 0 & 0 \\
\hline Polímeros & 17 & 0 & 0 & 0 & 0 \\
\hline Hornos & 0 & 0 & 42 & 0 & 0 \\
\hline Aeroespacio/Defensa & 0 & 0 & 13 & 0 & 0 \\
\hline Automoción & 0 & 0 & 0 & 0 & 0 \\
\hline Otros & 40 & 40 & 1045 & 20 & 0 \\
\hline Total & 1833 & 696 & 1438 & 402 & 438 \\
\hline
\end{tabular}


El Cuadro 2.2 [37] muestra el número de aplicaciones de cada empresa. Se puede apreciar que el DMC (Aspentech) es, con diferencia, el algoritmo más utilizado.

A pesar del gran número de aplicaciones, los productos actuales de control predictivo presentan varias limitaciones:

- Usan modelos sobreparametizados. La mayoría de aplicaciones industriales usan modelos de respuesta a impulso o escalón. Estos modelos suelen estar sobreparametrizados. No es raro que requieran entra 30 y 50 coeficientes para describir la dinámica del sistema. Además, no son válidos para procesos inestables.

- No existe un procedimiento universal para el ajuste del controlador, a pesar de su importancia para conseguir la estabilidad. En presencia de restricciones este ajuste se vuelve más complicado todavía, y no es fácil garantizar la estabilidad en lazo cerrado. Esto obliga a realizar a invertir mucho tiempo en simulaciones.

- Para acelerar el cálculo, varios paquetes ofrecen la posibilidad de calcular una solución sub-óptima. Esto es aceptable en aplicaciones de alta velocidad, pero no en la industria de procesos si no se garantiza que la solución sub-óptima está muy cercana a la óptima.

- Incertidumbre del modelo. A pesar de que todos los paquetes proporcionan una estimación de la incertidumbre del modelo, solo el RMPCT usa esta información en el diseño del controlador.

- Considerar que las perturbaciones son constantes. Aunque es una suposición razonable, una mejor caracterización de las perturbaciones mejoraría la realimentación.

- Un análisis sistemático de la robustez y la estabilidad no es posible con formulación de horizonte finito. La ley de control suele variar con el tiempo y es difícil representarla en forma de lazo cerrado, sobre todo en el caso con restricciones. Además los resultados obtenidos por la comunidad académica para estabilidad y robustez solo pueden aplicarse a procesos pequeños (en términos de espacio de estado y horizonte de control).

\subsection{Ajuste de parámetros del MPC}

\subsubsection{Resumen de parámetros del MPC}

De las secciones anteriores se pueden deducir los siguientes parámetros:

- Tiempo de muestreo (T)

- Horizonte de predicción (Pr):Indica el intervalo (expresado en instantes de muestreo) durante el cual la salida debe seguir a la referencia. También puede expresarse como el período comprendido entre $\mathrm{N}_{1}$ y $\mathrm{N}_{2}$. 
- Tiempo de predicción $(\operatorname{Pr} \times \mathrm{T})$ : Indica el horizonte de tiempo durante el cual la salida debe seguir a la referencia. O, dicho de otra forma, es el horizonte de predicción expresado en unidades de tiempo.

- Horizonte de control (M): Indica los intervalos futuros para los que se calculan los incrementos de control.

- Horizonte de modelo $\left(\mathrm{n}_{\mathrm{g}}\right)$ : Indica el intervalo de tiempo de la respuesta a escalón que se usa como modelo.

- factor de peso $(\lambda)$ : Este coeficiente penaliza la señal de control para evitar que sea demasiado agresiva y que haya oscilaciones en la respuesta. También tiene el efecto de dar robustez matemática al método minimizando el efecto de tener una matriz $\left(\mathbf{G}^{\mathbf{T}} \mathbf{G}+\lambda \mathbf{I}\right)$ no invertible.

\subsubsection{Estado del arte del ajuste de parámetros}

En la sección 1.1.1 se ha explicado la importancia y la dificultad de realizar un correcto ajuste de parámetros.

Algunos trabajos afrontan este reto desde una perspectiva analítica. Un algoritmo muy conocido es el de Shrindar y Cooper [16], [17] que presentan un método para calcular el factor de peso minimizando el número de condición de la matriz del sistema para sistemas MIMO y SISO sin restricciones aproximado por un sistema de primer orden con retraso (First Order plus Dead Time, FODT). Es uno de los primeros métodos, de los más extendidos y ha sido estudiado por varios investigadores, ([38], [39]). Otro ejemplo es el algoritmo presentado por Trierweiller y Farina [40] que usa un índice de robustez (Robustness Performance Number, RPN) que indica lo difícil que es para un sistema alcanzar las prestaciones requeridas con robustez. Este método da directrices para calcular el horizonte de predicción, el horizonte de control y el tiempo de muestreo. Calcula la matriz de pesos del sistema basándose en el RPN y modifica la función normal de coste cuando factoriza la matriz del sistema. Han, Zhao and Qian [41] proponen un algoritmo de minimizaciónmaximización sobre un índice de prestaciones. Garriga and Soroush proponen un ajuste mediante disposición de valores propios [42].

Los métodos mencionados anteriormente tienen en común que requieren para su desarrollo, compresión e implementación complejos conocimientos de análisis matemático y de teoría del control, lo que dificulta su implementación en la industria.

Otros trabajos abordan el problema desde un punto de vista basado en la práctica, usando reglas dadas por la experiencia y simulación de controladores reales. Este método es más común en la industria. Los trabajo de Iglesias et al [18] y Camacho et al [43] son un ejemplo de esto.Se presenta una fórmula obtenida por correlación con datos obtenidos de varias simulaciones. Bagheri y Khaki-Sedigh ([44]) proponen un análisis de la varianza . Wojsznis et al proponen el uso de métodos heurísticos [45].En esta categoría pueden 
incluirse los métodos de auto-ajuste ([46]).

Trabajos recientes introducen el uso de técnicas de control modernas para resolver el problema, como algoritmos genéticos y lógica difusa([47] [48]).

Sin embargo, a pesar de todos los trabajos anteriores a los largo de varias décadas el ajuste de parámetros del DMC no es un asunto ni mucho menos cerrado. Solo se ha estudiado con profundidad el caso monovariable sin resticciones.Para sistemas MIMO no hay tantos estudios([17] es de los pocos ejemplos que existen) y el ajuste en presencia de restricciones se basa en reglas dadas por la experiencia obtenida en simulaciones de problemas típicos. También se cuentan entre los estudios para MIMO los trabajos de Sivakumar [48] y Mishra et al [49], aunque debe aclararse que están orientados al diseño de MPC para columnas de destilación binarias y no intentan conseguir un método de ajuste universal.

Si tenemos en cuenta que el mayor éxito del MPC ha sido precisamente su capacidad para controlar procesos con múltiples entradas y salidas teniendo en cuenta restricciones, aún se está muy lejos de tener un método de ajuste que pueda ser empleado universalmente en aplicaciones industriales. 


\section{Capítulo 3}

\section{Reglas de diseño para sistemas SISO}

\subsection{Introducción}

El objetivo de este capítulo es desarrollar una serie de reglas de sintonía para el controlador predictivo DMC sin restricciones en el caso SISO. Los pasos que se seguirán son los siguientes:

- Se seleccionará un benchmark de la literatura y se obtendrá su equivalente e primer orden con retardo (First Order Plus Death Time, FOPDT). De esta forma los resultados de este estudio se podrán extender a sistemas que pueden simplificarse mediante un FOPDT. Esta simplificación ya ha sido usada con éxito por otros investigadores [16].

- Se calculará el equivalente LTI del DMC sin restricciones. Con este paso se obtendrá un sistema de bloques equivalente en tiempo discreto cuyos polos pueden calcularse.

- Una vez calculado el equivalente LTI, se estudiará el efecto de los polos en los parámetros realizando simulaciones en las que se mantendrán fijos todos los parámetros excepto el que se quiera estudiar.

- Con las conclusiones de las simulaciones, se deducirán una serie de reglas de sintonía.

- Las reglas de sintonía se pondrán a prueba ajustando el control con DMC de varios Benchmark extraídos de la literatura y una maqueta térmica real. Se comparará el método con otras reglas de sintonía existentes.

\subsection{Formulación LTI para DMC en el caso SISO}

En esta sección se demostrará la conveniencia de poder expresar el DMC con formulaciones alternativas: 
- Formulación Lineal Invariante en el Tiempo (Linear Time Invariant, LTI). Se trata de una representación con matrices y bloques. El DMC se representa como el sistema de la Figura 3.1, donde T,R y S son polinomios en $\mathrm{z}^{-1}, \Delta$ es el polinomio incremento $\left(\Delta=1-\mathrm{z}^{-1}\right)$ y $\mathrm{G}$ es la función de transferencia de la planta. Esta formulación ya ha sido planteada con anterioridad $([14],[15])$ para el control predictivo SISO.

- Función de transferencia: En esta representación la entrada del sistema (generalmente, una referencia, w) y la salida están relacionadas por una función de transferencia equivalente, $\mathrm{Y}=\mathrm{Hw}$. Esta representación permite llevar a cabo métodos de análisis de sistemas (como localización de polos y ceros y análisis de estabilidad) con el DMC.

Ambas formulaciones tienen propiedades analíticas propias y comunes y es posible convertir una representación en otra.

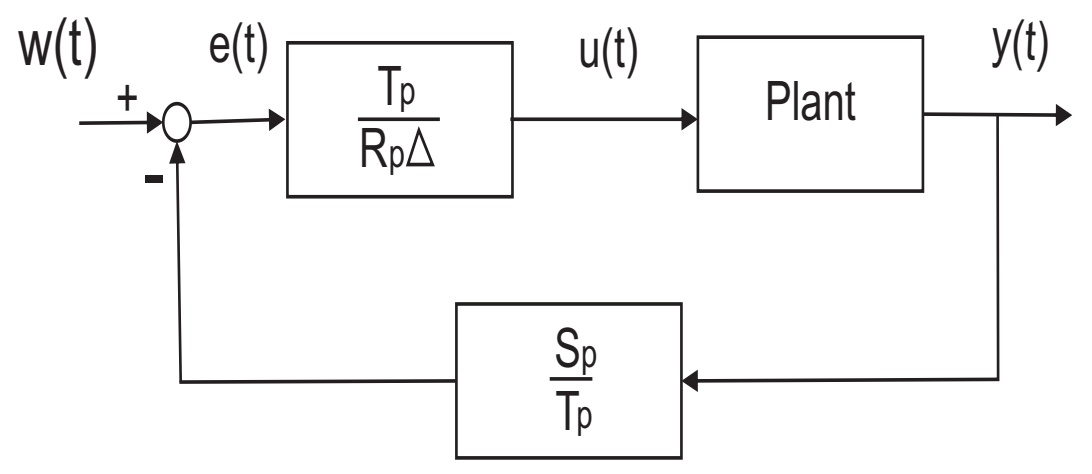

Figura 3.1: DMC con formulación LTI

Ahora, se va a hallar el equivalente LTI. La predicción de la salida será:

$$
\hat{\mathrm{y}}(\mathrm{t}+\mathrm{k})=\mathrm{G} \Delta \mathrm{u}(\mathrm{t}+\mathrm{k})+\mathrm{f}(\mathrm{t}+\mathrm{k})
$$

El primer componente del vector de incrementos de control óptimos es:

$$
\Delta \mathrm{u}_{\mathrm{opt}}=\mathbf{K e}
$$

Donde e es el vector de errores. No se tienen en cuenta las perturbaciones medibles y puede escribirse K como:

$$
\mathbf{K}=\left[\mathrm{k}_{1} \mathrm{k}_{2} \ldots \mathrm{k}_{\mathrm{Pr}}\right]=[1,0, \ldots, 0]\left(\mathbf{G}^{\mathbf{T}} \mathbf{G}+\lambda \mathbf{I}\right)^{-\mathbf{1}} \mathbf{G}^{\mathbf{T}}
$$

Desarrollando el valor del incremento de control óptimo y teniendo en cuenta una formulación alternativa para la respuesta libre:

$$
\mathrm{f}(\mathrm{t}+\mathrm{k})=\mathrm{y}_{\mathrm{m}}(\mathrm{t})+\sum_{\mathrm{i}=1}^{\mathrm{N}} \mathrm{s}_{\mathrm{n}}^{\mathrm{k}} \Delta \mathrm{u}(\mathrm{t}-\mathrm{i})
$$




$$
\begin{gathered}
S_{n}^{\mathrm{k}}\left(\mathrm{q}^{-1}\right)=\mathrm{s}_{1}^{\mathrm{k}} \mathrm{q}^{-1}+\mathrm{s}_{2}^{\mathrm{k}} \mathrm{q}^{-2}+\ldots+\mathrm{s}_{\mathrm{n}}^{\mathrm{k}} \mathrm{q}^{-\mathrm{n}} \\
\mathrm{s}_{\mathrm{n}}^{\mathrm{k}}=\mathrm{g}_{\mathrm{n}+\mathrm{k}}-\mathrm{g}_{\mathrm{n}} \\
\Delta \mathrm{u}_{\mathrm{opt}}(\mathrm{t})=\sum_{\mathrm{t}=1}^{\operatorname{Pr}} \mathrm{k}_{\mathrm{i}} \mathrm{w}(\mathrm{t}+\mathrm{i})-\sum_{\mathrm{t}=1}^{\operatorname{Pr}} \mathrm{k}_{\mathrm{i}} \mathrm{y}(\mathrm{t})-\sum_{\mathrm{t}=1}^{\operatorname{Pr}} \mathrm{k}_{\mathrm{i}} \mathrm{S}_{\mathrm{n}}^{\mathrm{i}} \Delta \mathrm{u}(\mathrm{t})
\end{gathered}
$$

Comparando esta expresión con un controlador de dos grados de libertad (ver Figura 3.1):

$$
\mathrm{R}_{\mathrm{p}}\left(\mathrm{z}^{-1}\right) \Delta \mathrm{u}(\mathrm{t})=\mathrm{T}_{\mathrm{p}}\left(\mathrm{z}^{-1}\right) \mathrm{w}(\mathrm{t})-\mathrm{S}_{\mathrm{p}}\left(\mathrm{z}^{-1}\right) \mathrm{y}(\mathrm{t})
$$

Se puede, entonces, despejar la expresión para cada polinomio:

$$
\begin{aligned}
& \mathrm{R}_{\mathrm{P}}=1+\sum_{\mathrm{i}=1}^{\mathrm{Pr}} \mathrm{k}_{\mathrm{i}} \mathrm{S}_{\mathrm{n}}^{\mathrm{i}} \\
& \mathrm{T}_{\mathrm{P}}=\sum_{\mathrm{i}=1}^{\operatorname{Pr}} \mathrm{k}_{\mathrm{i}} \mathrm{z}^{\mathrm{i}} \\
& \mathrm{S}_{\mathrm{P}}=\sum_{\mathrm{i}=1}^{\mathrm{Pr}} \mathrm{k}_{\mathrm{i}}
\end{aligned}
$$

Una vez todos los bloques han sido calculados, es posible expresar todo el sistema como una única función de transferencia equivalente, Figura 3.2.

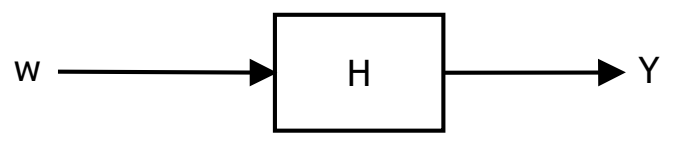

Figura 3.2: DMC SISO como sistema en lazo abierto

\subsection{Polos en lazo cerrado y su efecto en la respuesta tem- poral}

En esta sección se va a realizar un estudio del efecto de los parámetros del DMC en la posición de sus polos en lazo cerrado(expresado en formulación LTI) y, por consiguiente, en la respuesta temporal. Por lo tanto, resulta conveniente explicar como definen la respuesta temporal de un sistema en tiempo discreto y que posición debe buscarse. La formulación LTI del DMC suele resultar en sistemas de orden superior a 2, presentando parejas de polos complejos y polos reales. La respuesta temporal vendrá dada por la suma del efecto de todos los polos, siendo los polos dominantes (aquellos más lentos, es decir, los más cercanos al círculo unidad) los que dictaminan el tiempo de establecimiento. El 
efecto de los polos no dominantes en el tiempo de establecimiento y forma de la respuesta, puede despreciarse (pero siguen teniendo efecto en la ganancia del sistema).

Tal y como se ha indicado, existen varias localizaciones posibles. Cada localización de los polos (y su posible evolución) tienen un efecto propio en la respuesta temporal del sistema.

- Polos reales: Como puede verse en la Figura 3.3, pueden ser positivos o negativos.

- Los polos positivos producen una respuesta libre de oscilaciones. Cuanto mayor sea su valor absoluto, mayor será su tiempo de establecimiento (Figura 3.3 a).

- Los polos negativos producen respuestas oscilantes. Cuanto mayor sea su valor absoluto, mayores serán las oscilaciones al ir disminuyendo el factor de amortiguamiento (Figura $3.3 \mathrm{~b}$ ).

- Polos complejos conjugados: Los polos complejos conjugados siempre producen respuestas oscilantes (Figura 3.4). En general, cuanto mayor sea la parte imaginaria de un polo complejo, mayores serán las oscilaciones ya que el factor de amortiguamiento será cada vez menor, como queda reflejado en la Figura 3.4(a)(b). Sin embargo, debe tenerse en cuenta que es posible aumentar la parte imaginaria del polo y, a la vez, disminuir las oscilaciones, como sucede en la Figura 3.4(c), en la que, a pesar de aumentar la parte imaginaria, el sistema se mueve a una zona con mayor coeficiente de amortiguamiento y menores oscilaciones.

- Polos inestables: Los polos (reales o complejos) que estén fuera del círculo provocarán la inestabilidad del sistema.

Teniendo en cuenta el efecto de cada tipo de polo en la respuesta temporal, el objetivo de este capítulo es desarrollar una serie de reglas de sintonía que tengan el siguiente efecto en los polos:

1. Eliminen los polos inestables fuera del círculo unidad.

2. Hagan que los polos reales positivos sean dominantes para evitar las oscilaciones.

3. Minimicen la parte imaginaria de los polos complejos para reducir oscilaciones.

\subsection{Influencia de los parámetros del DMC en la respuesta del sistema}

Para estudiar el efecto del horizonte de predicción (Pr), tiempo de muestreo $(\mathrm{T})$, factor de peso $(\lambda)$, horizonte de control $(\mathrm{M})$ y horizonte de modelo $\left(\mathrm{n}_{\mathrm{g}}\right)$ en la respuesta temporal, se hará un análisis de los polos en lazo cerrado. Tal y como se ha explicado en la sección 3.2 un benchmark controlado mediante DMC se puede expresar como un sistema LTI, 


\subsection{INFLUENCIA DE LOS PARÁMETROS DEL DMC EN LA RESPUESTA DEL SISTEMA31}

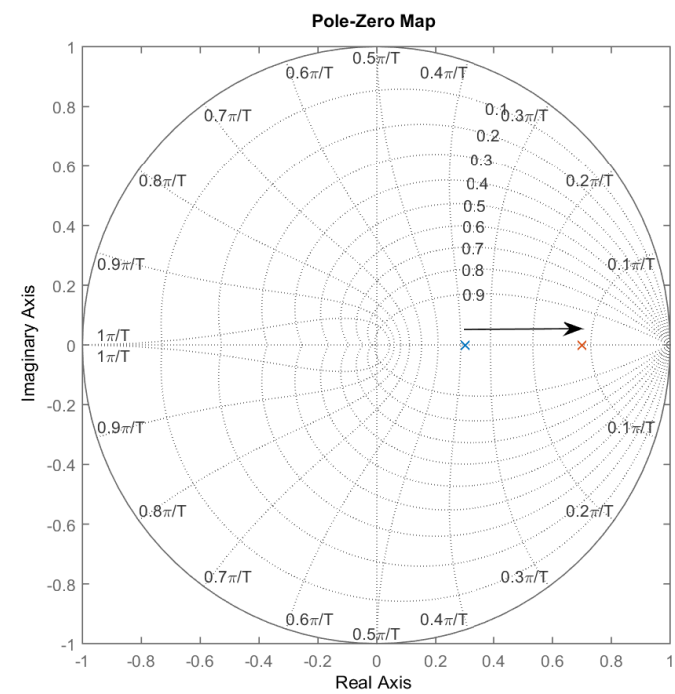

Localización (a)

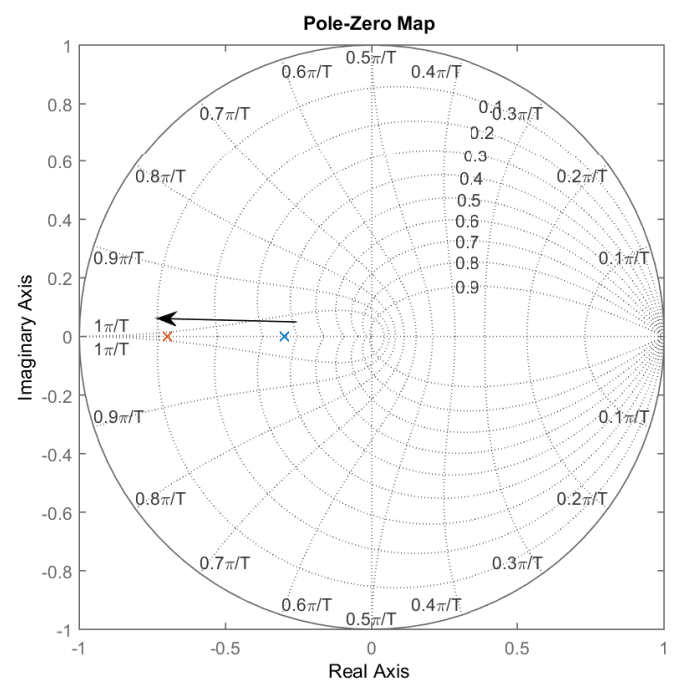

Localización (b)

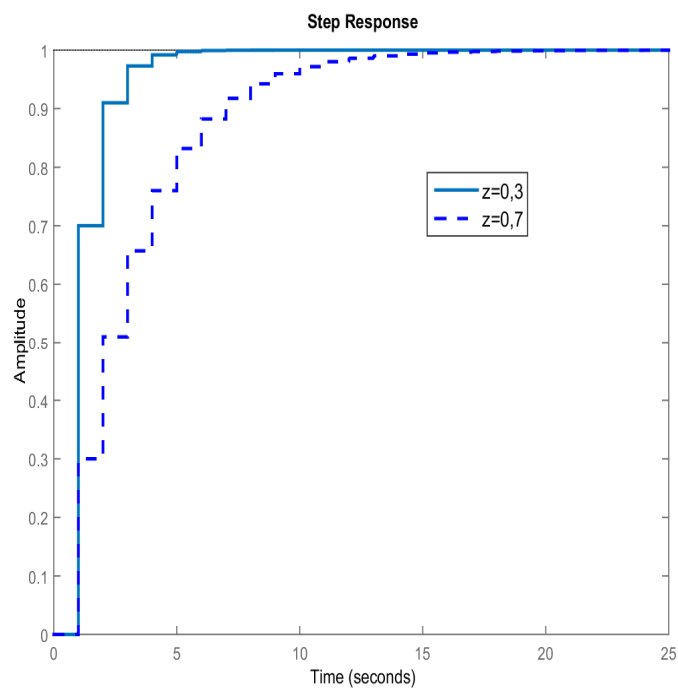

Respuesta(a)

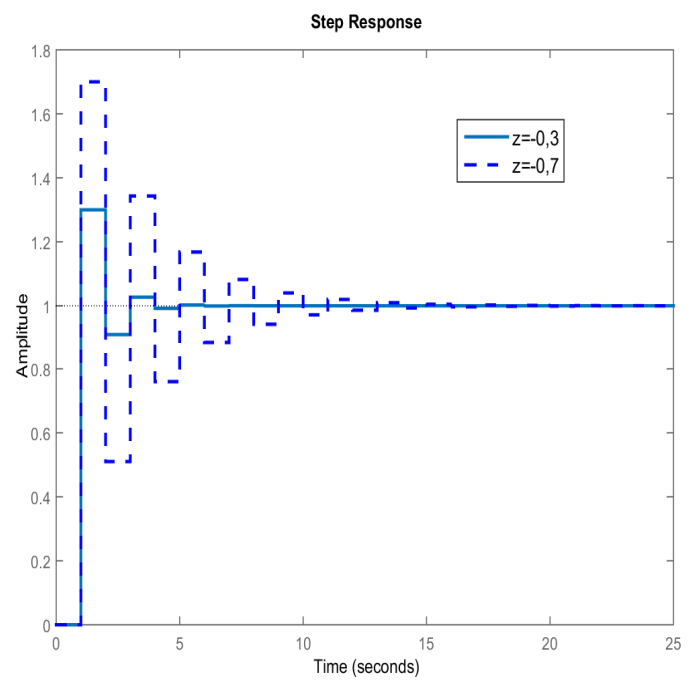

Respuesta (b)

Figura 3.3: Respuesta a un polo simple real estable positivo (a) y negativo (b) 


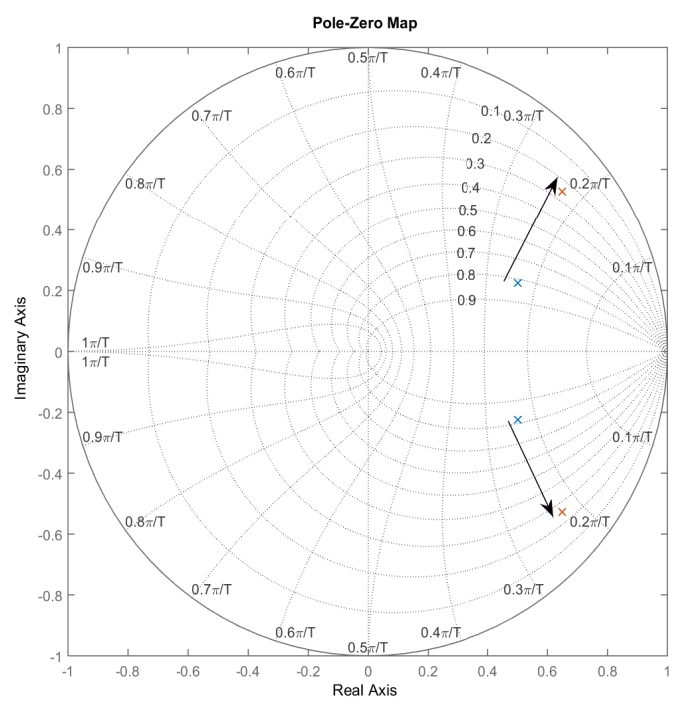

Localización (a)

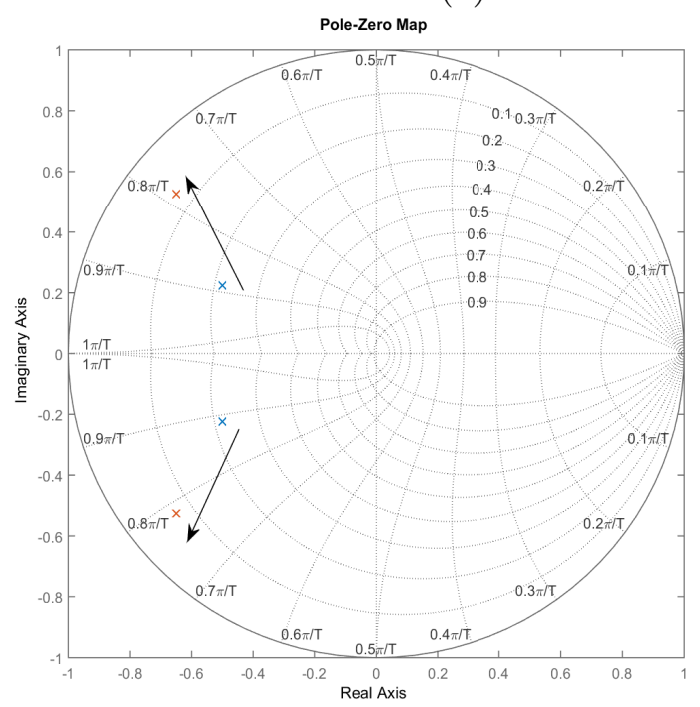

Localización (b)

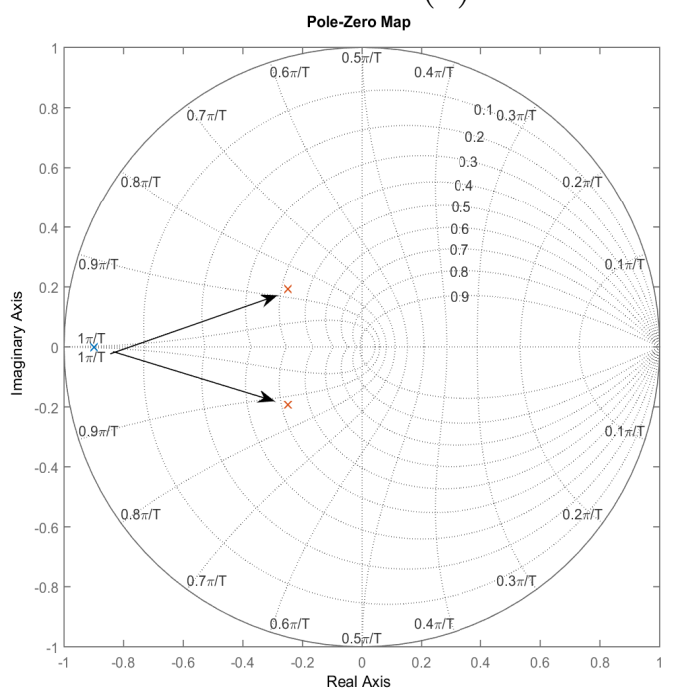

Localización (c)

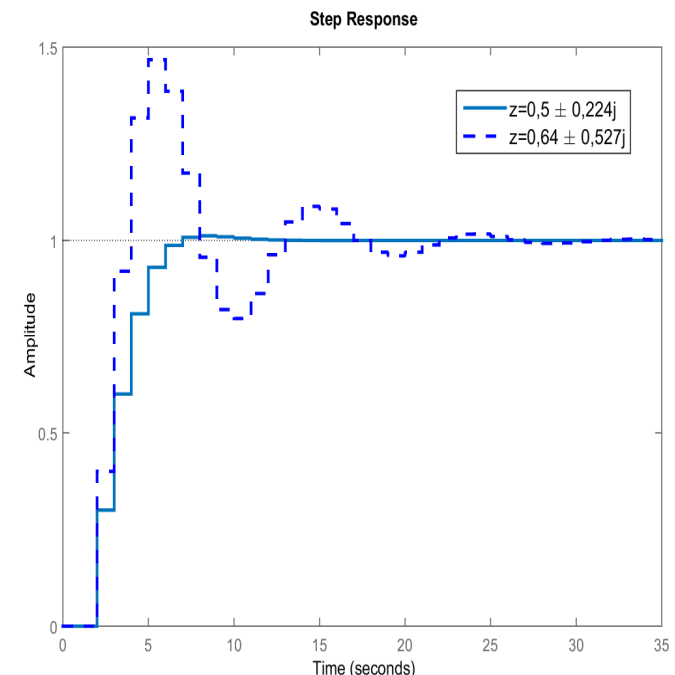

Respuesta (a)

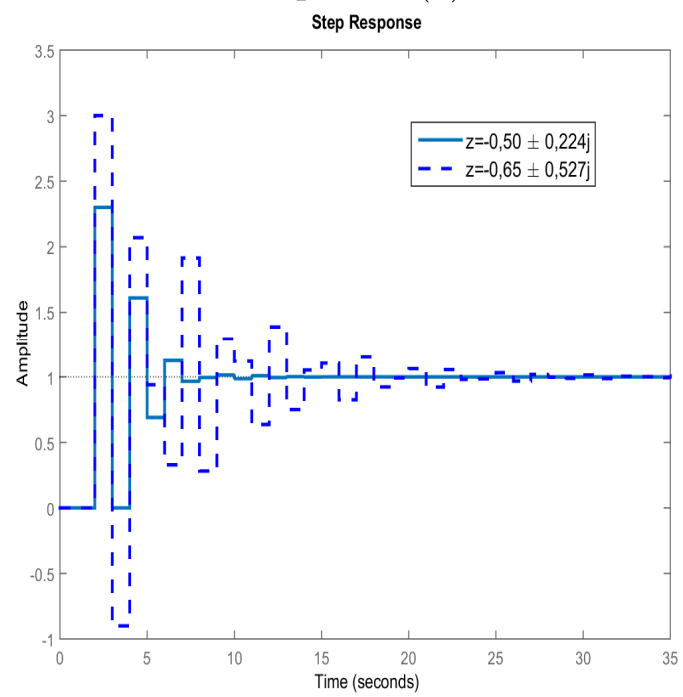

Respuesta (b)

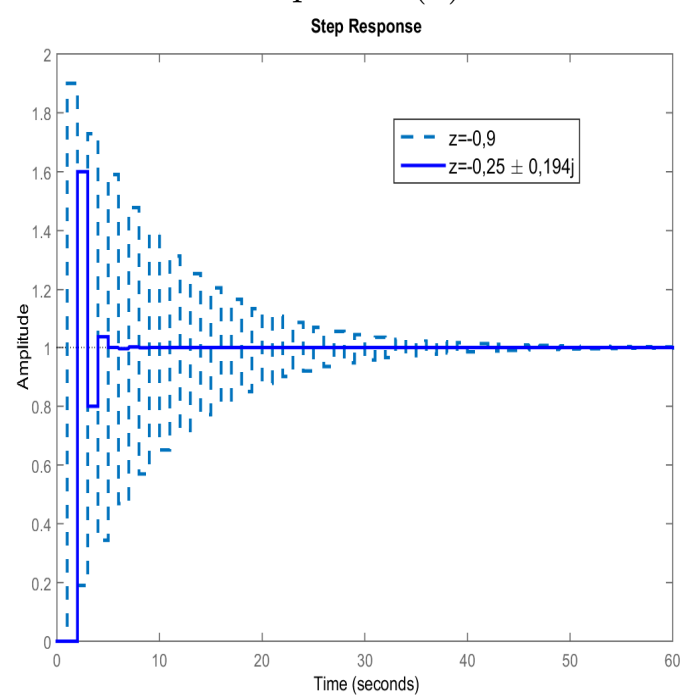

Respuesta (c)

Figura 3.4: Respuesta a una pareja de polos complejos estables con parte real positiva (a) y negativa(b)(c) 
cuyos polos pueden calcularse. Estos polos dependerán de los valores de los parámetros del DMC y los coeficientes de la respuesta a escalón. Si el comportamiento de los polos cuando cambian dichos parámetros puede deducirse, se podrá entender su influencia en la respuesta a un escalón unidad. Si se conoce la influencia en la respuesta temporal, se podrán desarrollar reglas heurísticas (con la misma filosofía que el método de ZieglerNichols para controladores PID, [2]). Se ha seleccionado el primer proceso usado en [16] (con un retraso de 16 segundos) para estudiar el movimiento de los polos:

$$
\mathrm{G}(\mathrm{s})=\frac{\mathrm{e}^{-16 \mathrm{~s}}}{(150 \mathrm{~s}+1)(24 \mathrm{~s}+1)}
$$

Las conclusiones sacadas del estudio del bechnmark de la Ecuación 3.12 deben ser aplicables a muchos sistemas. Este estudio no se hará en el bechnmark «tal cual», sino a su equivalente de primer orden con retraso (First Order Plus Dead Time, FOPDT, Ecuación 3.13 ). Esto asegurará que los resultados del sistema sean aplicables a otros sistemas de primer orden $\mathrm{y}$, por lo tanto, a cualquier sistema que se pueda simplificar mediante un FOPDT.

$$
\mathrm{G}(\mathrm{s})=\frac{\mathrm{e}^{-32 \mathrm{~s}}}{157 \mathrm{~s}+1}
$$

El análisis se hará para $\mathrm{T}=8$ segundos y $\mathrm{T}=16$ segundos . Estos tiempos de muestreo se han seleccionado de forma que el retraso de 32 segundos del equivalente FOPDT sea múltiplo de los mismos y lo más cercanos posibles a $0.05 \tau$ y $0.1 \tau$, tal y como recomiendan Shridhar y Cooper [16] ( $\tau$ es la constante de tiempo del equivalente FOPDT). Las Ecuaciones 3.14 y 3.15 son la forma discreta de la Ecuación 3.13 para unos tiempos de muestreo de 8 y 16 segundos respectivamente.

$$
\begin{aligned}
\mathrm{G}_{8}(\mathrm{z}) & =\frac{0.04968 \mathrm{z}^{-5}}{1-0.9503 \mathrm{z}^{-1}} \\
\mathrm{G}_{16}(\mathrm{z}) & =\frac{0.09516 \mathrm{z}^{-3}}{1-0.9048 \mathrm{z}^{-1}}
\end{aligned}
$$

Para realizar este estudio se hará un conjunto de simulaciones para cada parámetro, variando el mismo mientras que el resto se mantienen fijos en valores bajos para que asegurarse que su influencia no enmascare la del parámetro que se quiere estudiar.

\subsubsection{Influencia del horizonte de predicción y el tiempo de muestreo en la respuesta temporal.}

Tal y como se ha indicado antes, este análisis se realizará para $\mathrm{T}=8$ segundos y $\mathrm{T}=16$ segundos. Para cada tiempo de muestreo se variará el horizonte de predicción mientras el resto de parámetros se mantienen fijos $(\mathrm{M}=2$ y $\lambda=0,25)$.Los Cuadros 3.1 y 3.2 muestran los polos obtenidos para estos tiempos de muestreo. La Figura 3.5 permite observar la evolución de los polos del sistema conforme $\mathrm{T}$ y $\mathrm{Pr}$ cambian. En la Figura 3.5 cada polo tiene un símbolo dependiendo de su Pr. 


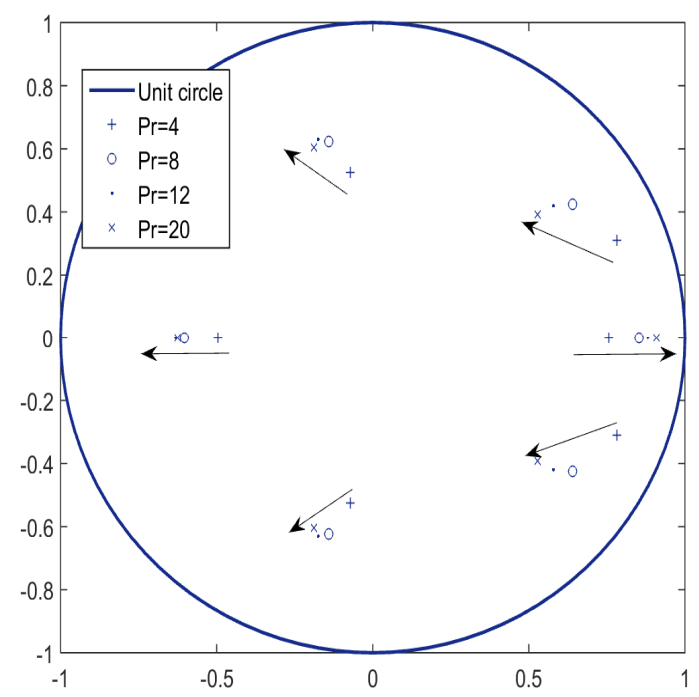

Polos en lazo cerrado para $\mathrm{T}=8$ segundos

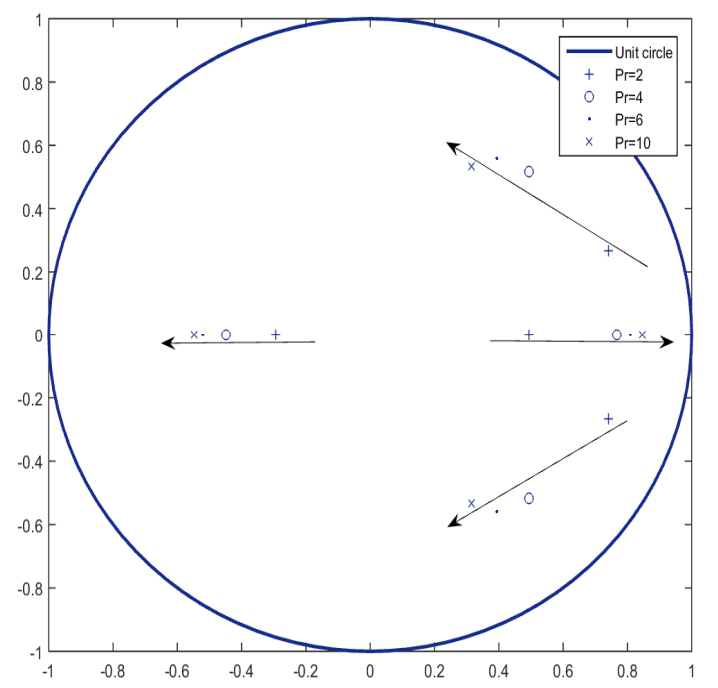

Polos en lazo cerrado para $\mathrm{T}=16$ segundos

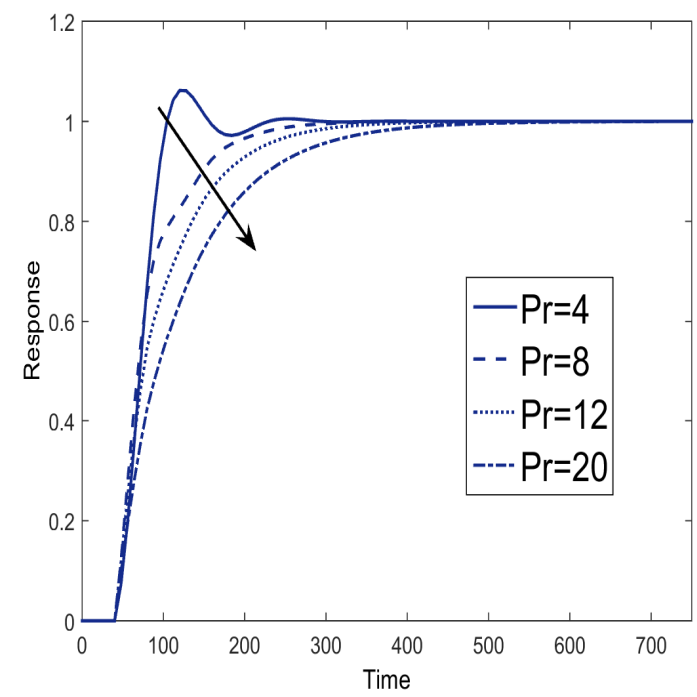

Respuesta temporal para $\mathrm{T}=8$ segundos y diferentes valores de $\mathrm{Pr}$

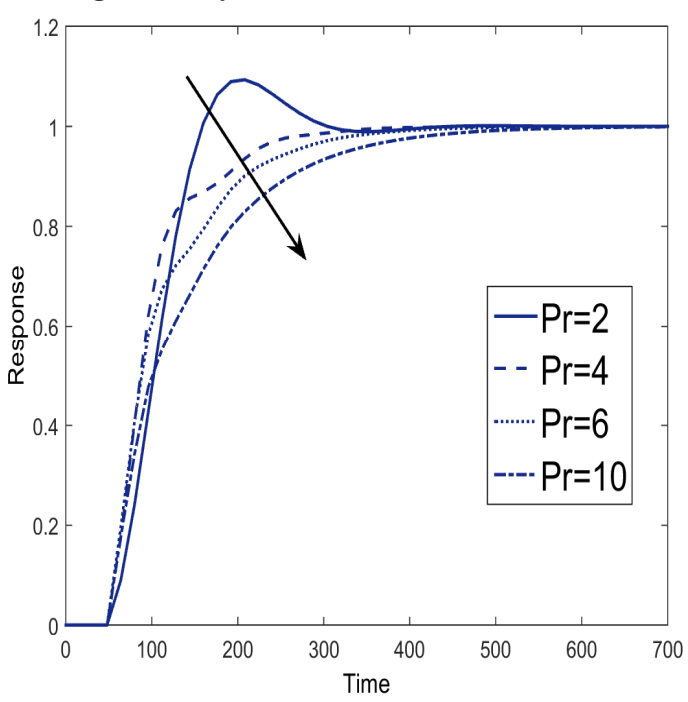

Respuesta temporal para $\mathrm{T}=16$ segundos y diferentes valores de $\operatorname{Pr}$

Figura 3.5: Localización de polos y respuesta temporal para $\mathrm{T}=8$ y $\mathrm{T}=16$ segundos 
Cuadro 3.1: Polos para $\mathrm{T}=8$ segundos

\begin{tabular}{|c|c|c|c|}
\hline $\operatorname{Pr}=4$ & $\operatorname{Pr}=8$ & $\operatorname{Pr}=12$ & $\operatorname{Pr}=20$ \\
\hline$-0,5$ & $-0,6$ & $-0,63$ & $-0,63$ \\
\hline$-0,0728+0,5242 \mathrm{i}$ & $-0,1402+0,6233 \mathrm{i}$ & $-0,1747+0,6303 \mathrm{i}$ & $-0,1887+0,6054 \mathrm{i}$ \\
\hline$-0,0728-0,5242 \mathrm{i}$ & $-0,1402-0,6233 \mathrm{i}$ & $-0,1747-0,6303 \mathrm{i}$ & $-0,1887-0,6054 \mathrm{i}$ \\
\hline $0,7814+0,3091 \mathrm{i}$ & $0,6406+0,4251 \mathrm{i}$ & $0,5787+0,4197 \mathrm{i}$ & $0,5275+0,3929 \mathrm{i}$ \\
\hline $0,7814-0,3091 \mathrm{i}$ & $0,6406-0,4251 \mathrm{i}$ & $0,5787-0,4197 \mathrm{i}$ & $0,5275-0,3929 \mathrm{i}$ \\
\hline 0,76 & 0,85 & 0,88 & 0,91 \\
\hline
\end{tabular}

Cuadro 3.2: Polos for $\mathrm{T}=16$ segundos

\begin{tabular}{|c|c|c|c|}
\hline $\operatorname{Pr}=2$ & $\operatorname{Pr}=4$ & $\operatorname{Pr}=6$ & $\operatorname{Pr}=10$ \\
\hline$-0,29$ & $-0,45$ & $-0,52$ & $-0,55$ \\
\hline $0,7418+0,2668 \mathrm{i}$ & $0,4938+0,5175 \mathrm{i}$ & $0,394+0,5588 \mathrm{i}$ & $0,3145+0,5327 \mathrm{i}$ \\
\hline $0,7418-0,2668 \mathrm{i}$ & $0,4938-0,5175 \mathrm{i}$ & $0,394-0,5588 \mathrm{i}$ & $0,3145-0,5327 \mathrm{i}$ \\
\hline 0,49 & 0,77 & 0,81 & 0,85 \\
\hline
\end{tabular}

Se ha seleccionado el horizonte de predicción de tal manera que el tiempo de predicción $(\operatorname{Pr} \times \mathrm{T})$ sea el mismo para ambos tiempos de muestreo y los resultados sean comparables. La primera conclusión que se saca de los Cuadros 3.1 y 3.2 y la Figura 3.5 es que al incrementar el horizonte de predicción:

- Aumenta el valor de los polos reales.

- Disminuye el módulo de los polos complejos, aunque aumenta si parte compleja.

Esto hace que que los polos reales positivos se conviertan en dominantes y dicten el comportamiento del sistema. Los polos positivos reales producen una respuesta sin oscilaciones. Por lo que si se requiere una respuesta libre de oscilaciones, incrementar $\operatorname{Pr}$ parece la elección adecuada. También puede concluirse que conforme crece el horizonte de predicción, su efecto en los polos se vuelve más débil y el comportamiento del sistema se aproxima al de un lazo abierto. Pr tiene un valor útil máximo e incrementar por encima de este valor no mejorará la respuesta del sistema, simplemente lo hará más lento.

La Figura 3.5 muestra que conforme se incrementa Pr, la respuesta del sistema se vuelve más lenta (se aproxima a un sistema en lazo abierto). Conforme el polo dominante se acerca al círculo unidad, el tiempo de respuesta del sistema se incrementa. Para obtener una respuesta libre de oscilaciones y, a la vez, un tiempo de de respuesta no demasiado lento, Pr debe elegirse con cuidado. La clave es usar un valor que haga que el polo real positivo sea claramente dominante. Para $\mathrm{T}=16$ segundos, este valor es $\operatorname{Pr}=6$ ( para este valor de $\mathrm{Pr}$, el módulo del polo complejo es 0.68 frente a 0.81 del polo real positivo). Para $\mathrm{T}=8$ segundos , sería $\mathrm{Pr}=8$ (El módulo del polo real es de 0.85 frente a 0.618 del polo complejo). 
Los Cuadros 3.1 y 3.2 muestran que cuanto menor es el tiempo de muestreo, más cerca están los polos del círculo unidad, lo cual es algo conocido [50]. Otro efecto es que los polos están más cerca los unos de los otros. Esto hace que para tiempos de muestreo pequeños, los polos complejos no sean tan dominantes, lo que disminuye las oscilaciones con respecto al mismo tiempo de predicción , pero mayor tiempo de muestreo.. Sin embargo esta disminución de oscilaciones viene a costa de un aumento en el tiempo de establecimiento (al acercarse los polos al círculo unidad). Además, conforme aumenta el horizonte de predicción y los polos reales se convierten en dominantes, la diferencia entre los polos calculados para los distintos tiempos de muestreo se vuelven difíciles de apreciar.

Como sucede en todo sistema en tiempo de discreto, la elección del tiempo de muestreo es un asunto delicado. Un tiempo de muestreo demasiado pequeño hará al sistema más lento de lo deseado. Si es demasiado grande, aparecerán oscilaciones debido a la incapacidad del sistema para reaccionar a perturbaciones. La experiencia ha demostrado que para MPC un valor adecuado es el indicado por Shridhar y Cooper [16]: El máximo valor entre $\mathrm{T} \leq 0,1 \tau_{\mathrm{p}}$ y $\mathrm{T} \leq 0,5 \Theta_{\mathrm{p}}$.

Es interesante hacer notar que para ambos tiempos de muestreo, el tiempo necesario para conseguir que el polo real positivo sea dominante es inferior a la constante de tiempo $(\tau=157)$. La constante de tiempo de un sistema nos dice la rapidez con la que un sistema reacciona. Como se indicó en la sección 2.5.1 el horizonte (o tiempo) de predicción indica el tiempo durante el cual la salida ha de seguir a la referencia. Cuanto más rápido sea un sistema, menos tiempo necesitará para reaccionar ante un cambio en la consigna y, por lo tanto, necesitará un tiempo de predicción $(\operatorname{Pr} \times \mathrm{T})$ menor. El horizonte de predicción está ligado al tiempo de muestreo y la constante de tiempo del sistema.

\subsubsection{Influencia del horizonte de control en la respuesta temporal.}

Trabajos anteriores (por ejemplo, Shridhar y Cooper [1]) indican que este parámetro tiene una influencia pequeña en la respuesta temporal. Se han realizado varias simulaciones variando el horizonte de control mientras que el resto de parámetros se mantienen constantes $(\mathrm{T}=16, \operatorname{Pr}=4$ y $\lambda=0.25)$. En el Cuadro 3.3 se muestran los polos obtenidos para el proceso de la Ecuación 3.15. Este Cuadro también indica los símbolos usados para cada valor de $\lambda$ en la Figura 3.6.

Cuadro 3.3: Polos para diferentes valores de $\mathrm{M}$

\begin{tabular}{|c|c|c|c|c|}
\hline $\mathrm{M}=1$ & $\mathrm{M}=2$ & $\mathrm{M}=3$ & $\mathrm{M}=4$ & $\mathrm{M}=5$ \\
\hline 0.7680 & 0.7536 & 0.7482 & 0.7475 & 0.7475 \\
\hline $0.4938+0.5175 \mathrm{i}$ & $0.5293+0.4624 \mathrm{i}$ & $0.5385+0.4505 \mathrm{i}$ & $0.5396+0.4494 \mathrm{i}$ & $0.5396+0.4494 \mathrm{i}$ \\
\hline $0.4938-0.5175 \mathrm{i}$ & $0.5293-0.4624 \mathrm{i}$ & $0.5385-0.4505 \mathrm{i}$ & $0.5396-0.4494 \mathrm{i}$ & $0.5396-0.4494 \mathrm{i}$ \\
\hline-0.4480 & -0.4049 & -0.3966 & -0.3958 & -0.3958 \\
\hline
\end{tabular}

Como puede verse en la Figura 3.6, los polos (y, por consiguiente, la respuesta temporal) varía muy poco al cambiar $M$. Puede verse que $M$ tiene una influencia limitada en el proceso, ya que la localización de los polos apenas cambia al variar este parámetro. El motivo es que la influencia de $\mathrm{M}$ depende de $\operatorname{Pr}$ y de $\lambda$. Veamos que ocurre cuando $\operatorname{Pr}$ 


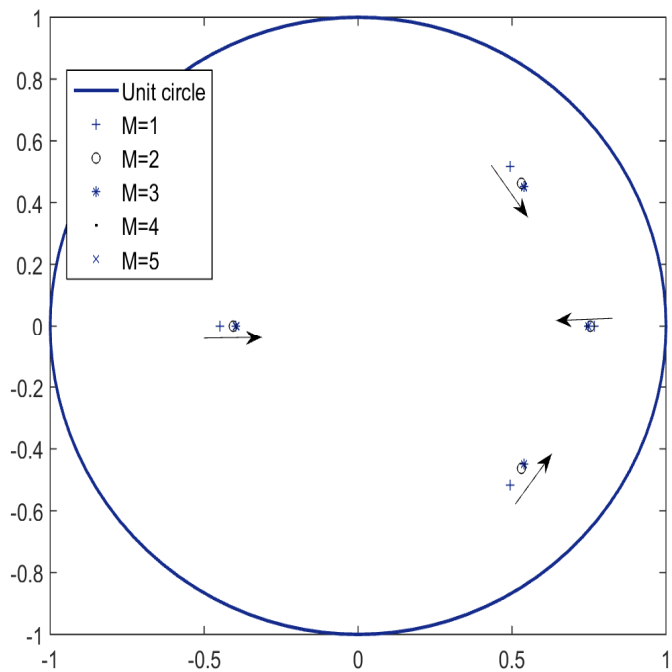

Efecto de $\mathrm{M}$ en los polos del sistema

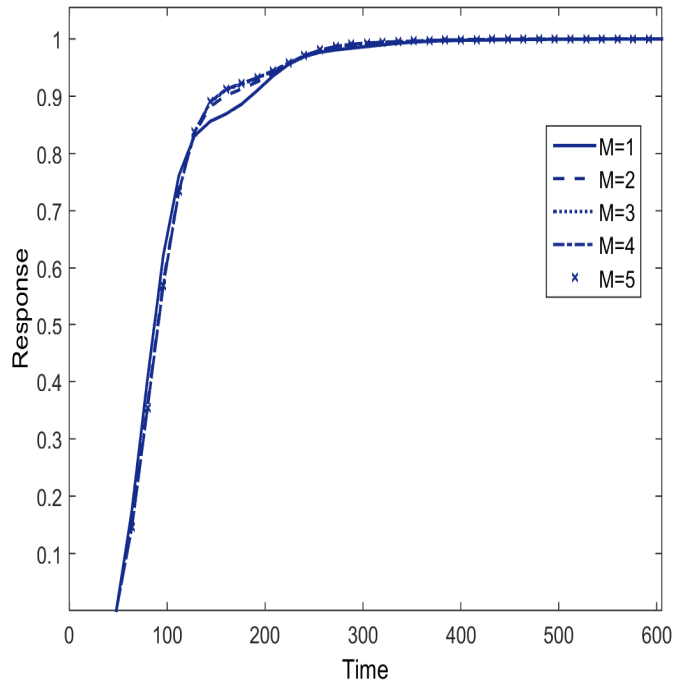

Respuesta temporal para varios valore de $\mathrm{M}$

Figura 3.6: Localización de polos y respuesta temporal para diferentes valores de $\mathrm{M}$

aumenta de $\operatorname{Pr}=4$ a distintos valores (Cuadros 3.4, 3.5 y 3.6 y Figura 3.7). Puede observarse que con valores de Pr superiores a la constante de tiempo del equivalente FOPDT, el efecto de $\mathrm{M}$ se vuelve más importante, reduciendo el valor del polo dominante y, por lo tanto, el tiempo de establecimiento. Sin embargo, debe tenerse en cuenta que los componentes reales e imaginarios de los polos complejos se incrementan y esto puede deteriorar la respuesta si estos polos se vuelven dominantes. Al alcanzar un determinado valor de $\mathrm{M}$, el efecto en la localización de los polos se vuelve más débil y solo se observan cambios menores al incrementar este parámetro.

Cuadro 3.4: Polos para diferentes valores de $\mathrm{M}$ y $\operatorname{Pr}=10$

\begin{tabular}{|c|c|c|c|c|}
\hline $\mathrm{M}=1$ & $\mathrm{M}=2$ & $\mathrm{M}=3$ & $\mathrm{M}=4$ & $\mathrm{M}=5$ \\
\hline 0.8478 & 0.8111 & 0.7830 & 0.7723 & 0.7698 \\
\hline $0.3084+0.5373 \mathrm{i}$ & $0.4401+0.4739 \mathrm{i}$ & $0.4856+0.4722 \mathrm{i}$ & $0.4985+0.4812 \mathrm{i}$ & $0.5009+0.4856 \mathrm{i}$ \\
\hline $0.3084-0.5373 \mathrm{i}$ & $0.4401-0.4739 \mathrm{i}$ & $0.4856-0.4722 \mathrm{i}$ & $0.4985-0.4812 \mathrm{i}$ & $0.5009-0.4856 \mathrm{i}$ \\
\hline-0.5578 & -0.4279 & -0.4177 & -0.4217 & -0.4242 \\
\hline
\end{tabular}

Cuadro 3.5: Polos para diferentes valores de $\mathrm{M}$ y $\mathrm{Pr}=60$

\begin{tabular}{|c|c|c|c|c|}
\hline $\mathrm{M}=1$ & $\mathrm{M}=2$ & $\mathrm{M}=3$ & $\mathrm{M}=4$ & $\mathrm{M}=5$ \\
\hline 0.9075 & 0.8105 & 0.7687 & 0.7650 & 0.7683 \\
\hline $0.2368+0.4291 \mathrm{i}$ & $0.4388+0.4788 \mathrm{i}$ & $0.4859+0.5369 \mathrm{i}$ & $0.4886+0.5474 \mathrm{i}$ & $0.4882+0.5323$ \\
\hline $0.2368-0.4291 \mathrm{i}$ & $0.4388-0.4788 \mathrm{i}$ & $0.4859-0.5369 \mathrm{i}$ & $0.4886-0.5474 \mathrm{i}$ & $0.4882-0.5323$ \\
\hline-0.4822 & -0.4321 & -0.4642 & -0.4712 & -0.4601 \\
\hline
\end{tabular}




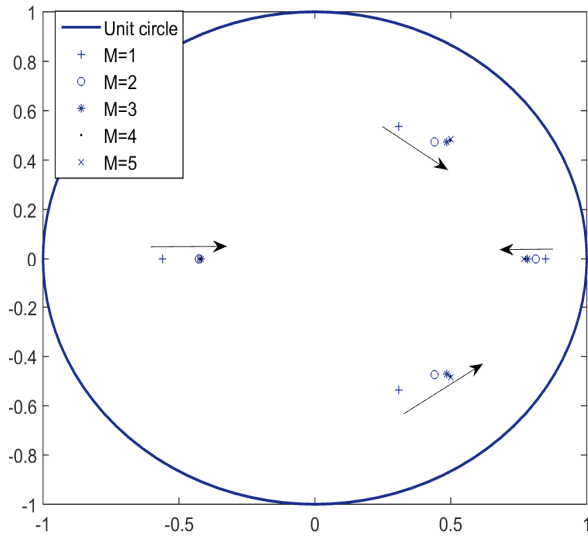

Efecto de $\mathrm{M}$ en los polos del sistema para $\operatorname{Pr}=10$

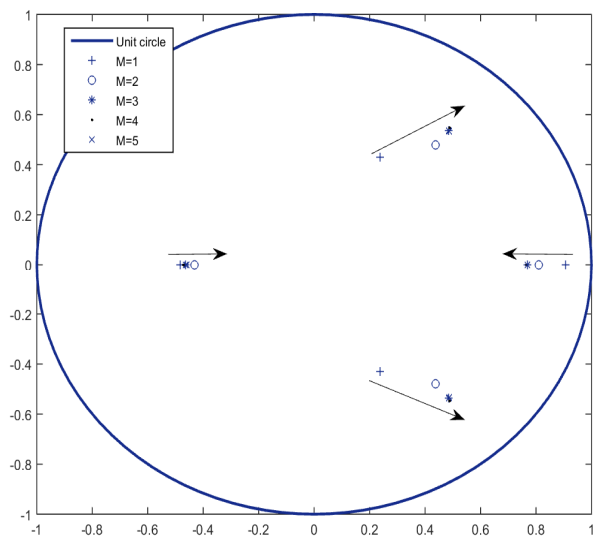

Efecto de $\mathrm{M}$ en los polos del sistema para $\operatorname{Pr}=60$

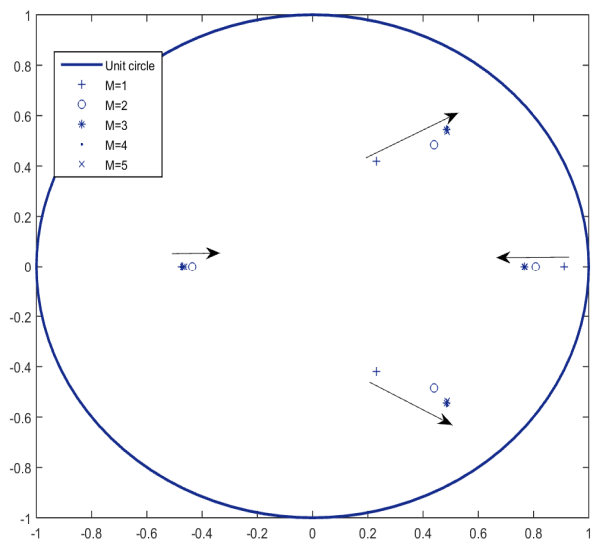

Efecto de $\mathrm{M}$ en los polos del sistema para $\mathrm{Pr}=120$

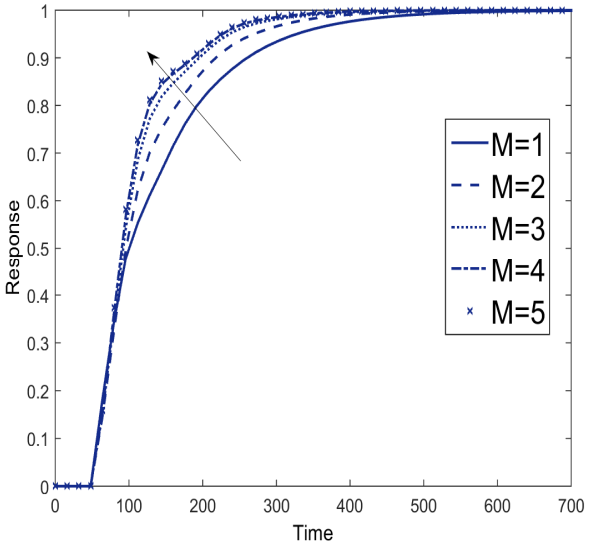

Respuesta temporal para varios valores de $\mathrm{M}$ y $\mathrm{Pr}=10$

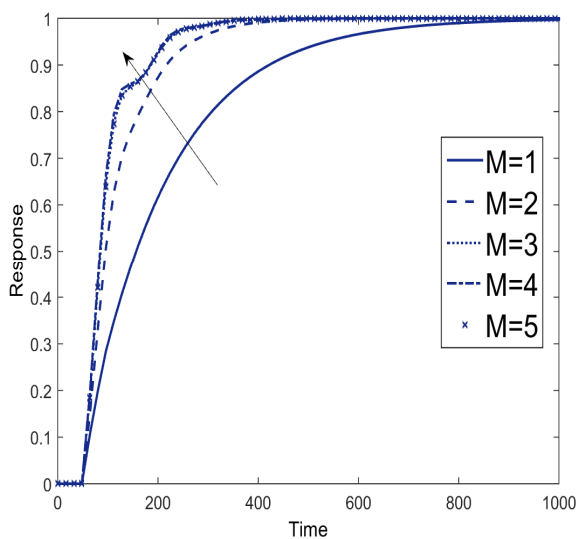

Respuesta temporal para varios valores de $\mathrm{M}$ y $\mathrm{Pr}=60$

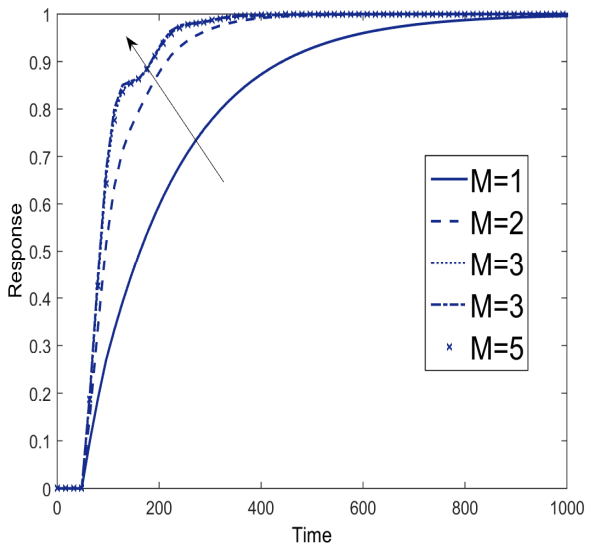

Respuesta temporal para varios valores de $\mathrm{M}$ y $\mathrm{Pr}=120$

Figura 3.7: Localización de polos y respuesta temporal variando Pr y M 
Cuadro 3.6: Polos para diferentes valores de $\mathrm{M}$ y $\operatorname{Pr}=120$

\begin{tabular}{|c|c|c|c|c|}
\hline $\mathrm{M}=1$ & $\mathrm{M}=2$ & $\mathrm{M}=3$ & $\mathrm{M}=4$ & $\mathrm{M}=5$ \\
\hline 0.9119 & 0.8084 & 0.7673 & 0.7646 & 0.7684 \\
\hline $0.2320+0.4202 \mathrm{i}$ & $0.4399+0.4842 \mathrm{i}$ & $0.4851+0.5456 \mathrm{i}$ & $0.4869+0.5536 \mathrm{i}$ & $0.4868+0.5356 \mathrm{i}$ \\
\hline $0.2320-0.4202 \mathrm{i}$ & $0.4399-0.4842 \mathrm{i}$ & $0.4851-0.5456 \mathrm{i}$ & $0.4869-0.5536 \mathrm{i}$ & $0.4868-0.5356 \mathrm{i}$ \\
\hline-0.4737 & -0.4362 & -0.4709 & -0.4763 & -0.4630 \\
\hline
\end{tabular}

\subsubsection{Influencia del factor de peso en la respuesta temporal}

El factor de peso tiene el efecto de suavizar la respuesta del sistema, pero lo hace de forma opuesta al horizonte de predicción. El Cuadro 3.7 y la Figura 3.8 (La flecha indica la dirección en la que se incrementa $\lambda$ ) muestran que conforme aumenta $\lambda$ (manteniendo el resto de parámetros fijos en $\operatorname{Pr}=2, \mathrm{M}=2$ y $\mathrm{T}=16$ ), el polo real positivo y la parte imaginaria de los polos complejos disminuyen mientras que la parte real del polo complejo aumenta. Esto puede llevar a que el polo complejo sea el dominante y que la respuesta presente sobrepicos y oscilaciones, como puede verse en la Figura 3.8. El efecto de suavizar la respuesta se explica por el hecho de que al aumentar la parte real y disminuir la parte imaginaria, el polo complejo se va aproximando a un polo real y su respuesta. Además, al aumentar el módulo del polo, se incrementa su tiempo de establecimiento. El efecto de este parámetro está condicionado por el horizonte de predicción . Su efecto se vuelve muy difícil de apreciar conforme Pr aumenta.

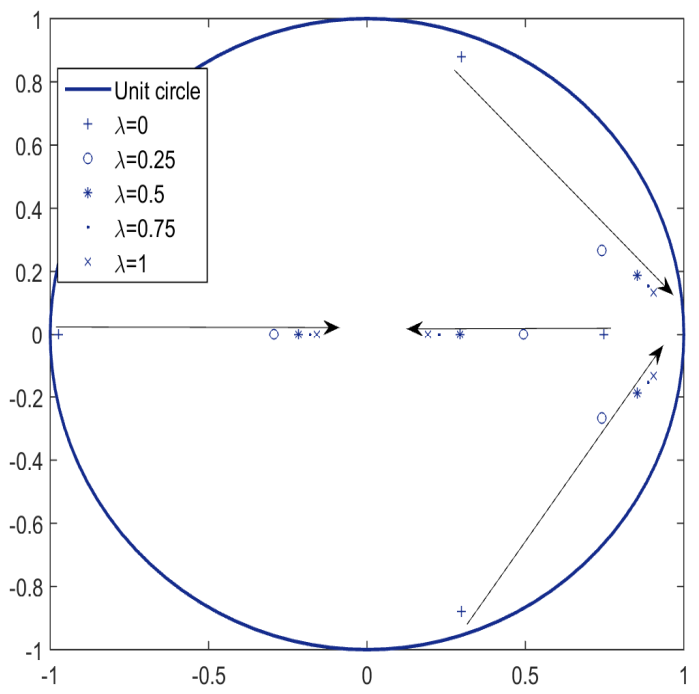

Evolución de polos con $\lambda(\operatorname{Pr}=2)$

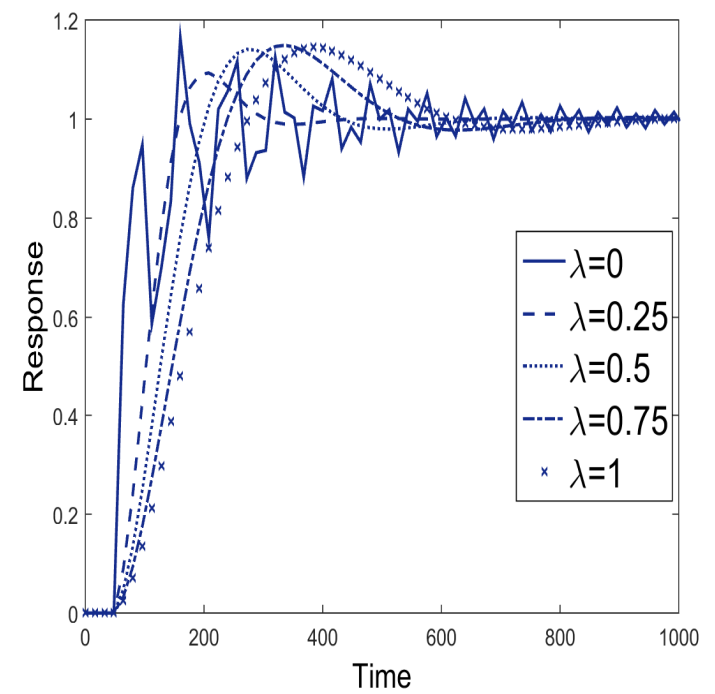

Respuesta temporal para varios valores de $\lambda$

Figura 3.8: Localización de polos y respuesta temporal para distintos valores de $\lambda$

El efecto de $\lambda$ está claro, ahora es necesario encontrar un valor adecuado. Como se ha visto en el Cuadro 3.7 y la Figura 3.8, el efecto de $\lambda$ se vuelve despreciable una vez que se ha alcanzado un determinado valor. Debe hacerse notar que la solución del DMC 
(Ecuación 4.4) puede resumirse como el producto de una matriz, $\mathbf{K}$, independiente del tiempo, y del vector de errores(e), que cambia en cada instante de muestreo(Ecuación 3.16). En el primer intervalo de muestreo el valor de los incrementos de control puede calcularse sin necesidad de ejecutar un proceso de simulación. Solo debe tenerse en cuenta que la respuesta libre, $\mathbf{f}$, en este primer intervalo es cero. Esto hace que el vector de errores sea igual a la referencia, que es un dato conocido.

$$
\Delta \mathbf{u}=\left(\mathbf{G}^{\mathbf{T}} \mathbf{G}+\lambda \mathbf{I}\right)^{-\mathbf{1}} \mathbf{G}^{\mathbf{T}}(\mathbf{w}-\mathbf{f})=\mathbf{K}(\mathbf{w}-\mathbf{f})=\mathbf{K e}
$$

Este valor del primer incremento de control puede calcularse para varios valores de $\lambda$ y puede trazarse una curva. Para valores muy pequeños de $\lambda$ el primer incremento de control toma valores muy altos, lo que apunta a una respuesta inestable. Para valores altos de $\lambda$, el valor del incremento de control disminuye muy lentamente (esto concuerda con los resultados obtenidos anteriormente). Hay un rango de valores de $\lambda$ en el que los incrementos de control cambian significativamente. El factor de peso debería estar en ese rango.

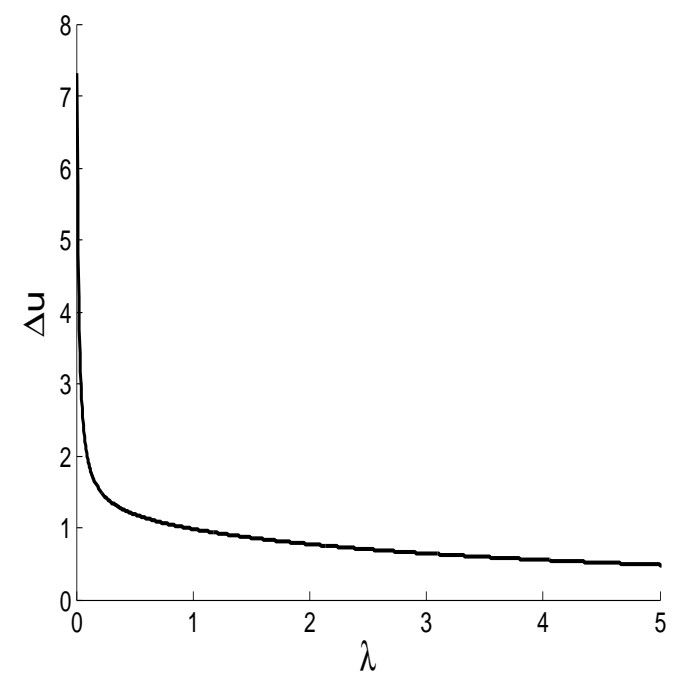

Figura 3.9: Curva de incrementos de control-factor de peso

En la Figura 3.9 se muestra la relación entre el factor de peso y el primer incremento de control para unos valores fijos de los horizontes de predicción y control. Se puede comprobar que, tal y como se esperaba, el valor del primer incremento de control decrece conforme $\lambda$ aumenta. También se llega a un punto a partir del cual incrementar $\lambda$ tiene un impacto pequeño en el primer incremento de control. Esta curva muestra que para los valores seleccionados para el resto de los parámetros, el valor máximo útil de $\lambda$ es 0,25.

El factor de peso también está relacionado con la robustez matemática del algoritmo. Como puede verse en la Ecuación 4.4, si $\lambda$ toma un valor de cero, el valor del DMC depende de la matriz inversa de $\mathrm{G}^{\mathrm{T}} \mathrm{G}$. En el caso de que $\mathrm{G}^{\mathrm{T}} \mathrm{G}$ no sea invertible (su determinante es 
cero o cercano a cero), el sistema se volvería inestable. La Figura 3.10 muestra la evolución de este determinante con M y Pr para un valor fijo de $\lambda$. Puede verse que, si $\lambda$ es demasiado bajo (por ejemplo, cero para este gráfico), conforme $\mathrm{M}$ aumenta el valor del determinante de $\mathrm{G}^{\mathrm{T}} \mathrm{G}$ disminuye y podría llegar a cero, haciendo imposible calcular ningún incremento de control.

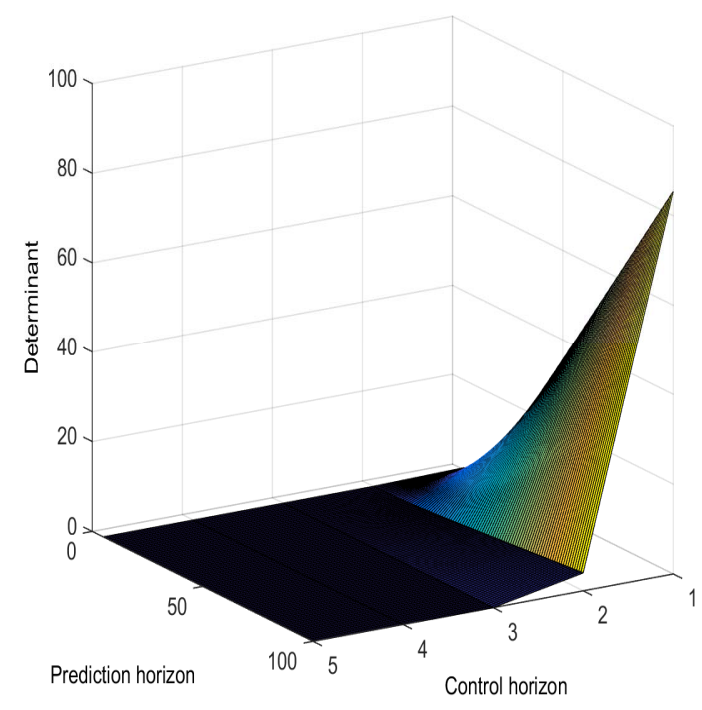

Figura 3.10: Determinante de $\mathrm{G}^{\mathrm{T}} \mathrm{G}$ para $\lambda=0$

\subsubsection{Influencia del horizonte de modelo en la respuesta temporal}

Este parámetro indica el tiempo de la respuesta a escalón usado como modelo, $\mathrm{n}_{\mathrm{g}}$ es el número de coeficientes de respuesta a escalón utilizados (g). La respuesta mejora incrementando $\mathrm{n}_{\mathrm{g}}$ hasta un punto en el que deja de tener influencia alguna. El horizonte de modelo se utiliza para calcular la respuesta libre del algoritmo del DMC, Ecuación (2.13). Cuando todos los coeficientes $g_{i+k}$ alcanzan el valor de $g_{i}$, este término se volverá cero. Todos los coeficientes g adicionales que incrementen el valor de $n_{g}$ por encima de este límite no tienen efecto. El DMC se aplica a procesos estables, en los que hay un valor de $n_{\mathrm{g}}$ a partir del cual $\mathrm{g}_{\mathrm{k}}-\mathrm{g}_{\mathrm{k}-1}=0$. Si este valor es el tiempo de establecimiento (n), entonces se debe cumplir que $\mathrm{n}_{\mathrm{g}}=\mathrm{n}+\operatorname{Pr}$. Luego $\mathrm{n}_{\mathrm{g}}$ depende del horizonte de predicción y el tiempo de muestreo. Para el proceso de ejemplo, el tiempo de establecimiento es de 950 segundos aproximadamente. Para un tiempo de muestreo de 16 segundos, esto significa que $\mathrm{n}=60$. Para $\operatorname{Pr}=4$, el $\mathrm{n}_{\mathrm{g}}$ ideal sería 84. La Figura 3.11 muestra la evolución de la respuesta del sistema desde $n_{\mathrm{g}}=15$ hasta $\mathrm{n}_{\mathrm{g}}=65$ para el DMC. Como puede verse solo valores pequeños de $n_{g}$ dan una respuesta inadecuada. A partir de $n_{g}>\frac{n}{2}$ la predicción de la respuesta es suficientemente precisa. 
Cuadro 3.7: Evolución de polos con $\lambda$

\begin{tabular}{|c|c|c|c|}
\hline \multicolumn{4}{|c|}{$\lambda=0$} \\
\hline $\operatorname{Pr}=2$ & $\operatorname{Pr}=4$ & $\mathrm{Pr}=6$ & $\mathrm{Pr}=10$ \\
\hline$-0,97$ & $-0,78$ & $-0,7$ & $-0,61$ \\
\hline $0,2983+0,8792 \mathrm{i}$ & $0,3089+0,7199 \mathrm{i}$ & $0,3001+0,6382 \mathrm{i}$ & $0,2815+0,5552 \mathrm{i}$ \\
\hline $0,2983-0,8792 \mathrm{i}$ & $0,3089-0,7199 \mathrm{i}$ & $0,3001-0,6382 \mathrm{i}$ & $0,2815-0,5552 \mathrm{i}$ \\
\hline 0,75 & 0,79 & 0,81 & 0,85 \\
\hline \multicolumn{4}{|c|}{$\lambda=0.25$} \\
\hline $\operatorname{Pr}=2$ & $\operatorname{Pr}=4$ & $\operatorname{Pr}=6$ & $\operatorname{Pr}=10$ \\
\hline$-0,29$ & $-0,45$ & $-0,52$ & $-0,55$ \\
\hline $0,7418+0,2668 \mathrm{i}$ & $0,4938+0,5175 \mathrm{i}$ & $0,394+0,5588 \mathrm{i}$ & $0,3145+0,5327 \mathrm{i}$ \\
\hline $0,7418-0,2668 \mathrm{i}$ & $0,4938-0,5175 \mathrm{i}$ & $0,394-0,5588 \mathrm{i}$ & $0,3145-0,5327 \mathrm{i}$ \\
\hline 0,49 & 0,77 & 0,81 & 0,85 \\
\hline 0 & 0 & 0 & 0 \\
\hline \multicolumn{4}{|c|}{$\lambda=0.50$} \\
\hline $\operatorname{Pr}=2$ & $\operatorname{Pr}=4$ & $\operatorname{Pr}=6$ & $\operatorname{Pr}=10$ \\
\hline$-0,22$ & $-0,35$ & $-0,43$ & $-0,5$ \\
\hline $0,854+0,1877 \mathrm{i}$ & $0,5646+0,3613 \mathrm{i}$ & $0,4494+0,4828 \mathrm{i}$ & $0,342+0,5091 \mathrm{i}$ \\
\hline $0,854-0,1877 \mathrm{i}$ & $0,5646-0,3613 \mathrm{i}$ & $0,4494-0,4828 \mathrm{i}$ & $0,342-0,5091 \mathrm{i}$ \\
\hline 0,29 & 0,73 & 0,8 & 0,85 \\
\hline 0 & 0 & 0 & 0 \\
\hline \multicolumn{4}{|c|}{$\lambda=0.75$} \\
\hline $\operatorname{Pr}=2$ & $\operatorname{Pr}=4$ & $\operatorname{Pr}=6$ & $\operatorname{Pr}=10$ \\
\hline$-0,18$ & $-0,3$ & $-0,38$ & $-0,46$ \\
\hline $0,8876+0,1544 \mathrm{i}$ & $0,6435+0,2185 \mathrm{i}$ & $0,4859+0,4139 \mathrm{i}$ & $0,3651+0,4851 \mathrm{i}$ \\
\hline $0,8876-0,1544 \mathrm{i}$ & $0,6435-0,2185 \mathrm{i}$ & $0,4859-0,4139 \mathrm{i}$ & $0,3651-0,4851 \mathrm{i}$ \\
\hline 0,23 & 0,62 & 0,8 & 0,85 \\
\hline 0 & 0 & 0 & 0 \\
\hline \multicolumn{4}{|c|}{$\lambda=1$} \\
\hline $\operatorname{Pr}=2$ & $\operatorname{Pr}=4$ & $\mathrm{Pr}=6$ & $\operatorname{Pr}=10$ \\
\hline$-0,16$ & $-0,26$ & $-0,34$ & $-0,43$ \\
\hline $0,904+0,1334 \mathrm{i}$ & $0,7524+0,1818 \mathrm{i}$ & $0,5129+0,3498 \mathrm{i}$ & $0,3847+0,4614 \mathrm{i}$ \\
\hline $0,904-0,1334 \mathrm{i}$ & $0,7524-0,1818 \mathrm{i}$ & $0,5129-0,3498 \mathrm{i}$ & $0,3847-0,4614 \mathrm{i}$ \\
\hline 0,19 & 0,43 & 0,79 & 0,84 \\
\hline 0 & 0 & 0 & 0 \\
\hline
\end{tabular}




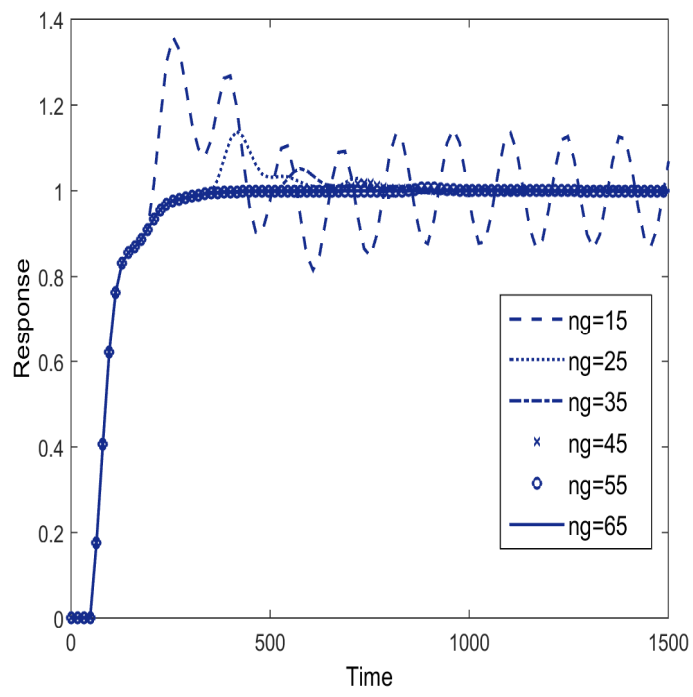

Respuesta temporal variando $\mathrm{n}_{\mathrm{g}}$

Figura 3.11: Respuesta temporal para varios valores de $n_{g}$

\subsubsection{Estabilidad del sistema}

A continuación se hará un estudio del impacto del factor de peso, horizonte de predicción y horizonte de control en la estabilidad del sistema. El factor de peso permite tener en cuenta la minimización de los incrementos de control como criterio en la función de coste, aparte del error de salida. Si $\lambda$ es igual a cero o demasiado pequeño, el DMC calculará un $\Delta \mathrm{u}$ que solo minimice el error de salida. Esto ocasionará una señal de control demasiado agresiva que podría inestabilizar el sistema. El Cuadro 3.8 y la Figura 3.12 muestran los polos del sistema para $\lambda=0, \operatorname{Pr}=100$ y varios valores de $\mathrm{M}$.

Cuadro 3.8: Polos para $\lambda=0$ y $\operatorname{Pr}=100$

\begin{tabular}{|c|c|c|c|}
\hline $\mathrm{M}=1$ & $\mathrm{M}=2$ & $\mathrm{M}=3$ & $\mathrm{M}=4$ \\
\hline-0.4764 & -1.2207 & -1.2207 & -1.2207 \\
\hline $0.2323+0.4221 \mathrm{i}$ & $0.2481+1.0340 \mathrm{i}$ & $0.2481+1.0340 \mathrm{i}$ & $0.2481+1.0340 \mathrm{i}$ \\
\hline $0.2323-0.4221 \mathrm{i}$ & $0.2481-1.0340 \mathrm{i}$ & $0.2481-1.0340 \mathrm{i}$ & $0.2481-1.0340 \mathrm{i}$ \\
\hline 0.9111 & 0.7245 & 0.7245 & 0.7245 \\
\hline
\end{tabular}

Los horizontes de predicción y control también tienen influencia en la estabilidad. Si el horizonte de predicción es muy grande, el término de errores en la función de coste será dominante y los incrementos de control resultantes podrían llegar a ser lo suficientemente grandes como para inestabilizar el sistema. El efecto del horizonte de predicción en la estabilidad puede verse en el Cuadro 3.9 y Figura 3.12, conforme Pr aumenta, el sistema se vuelve inestable.

Los horizontes de predicción y control tienden a inestabilizar el sistema si toman valores 


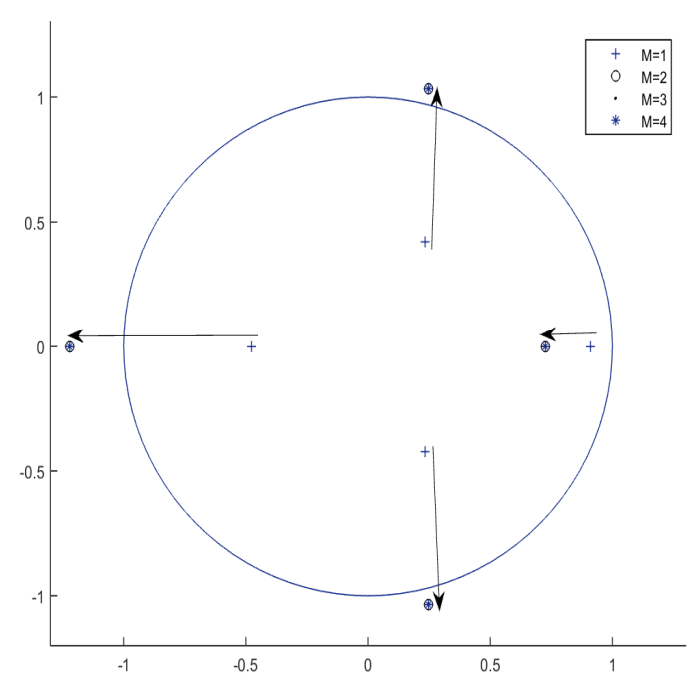

Polos variando $\mathrm{M}$

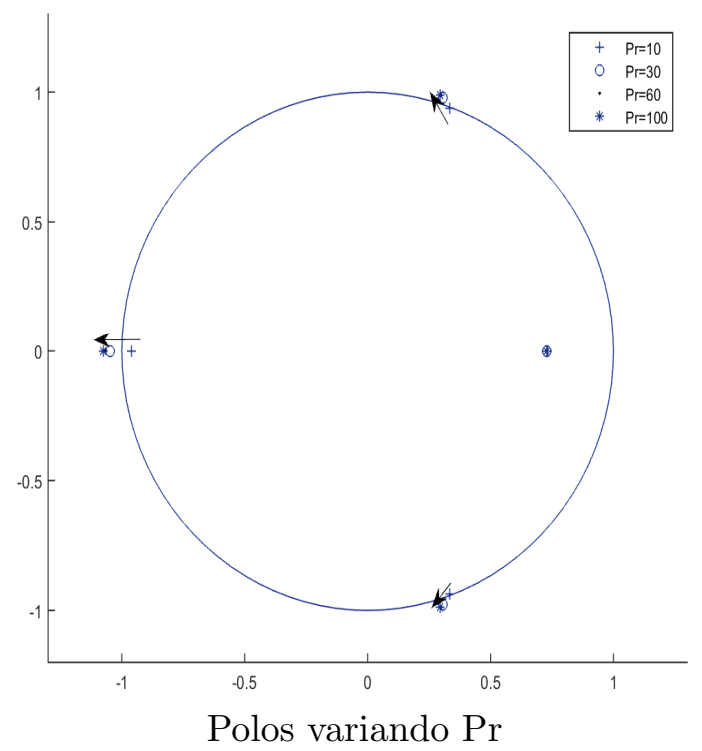

Polos variando $\mathrm{Pr}$

Figura 3.12: Polos para $\lambda=0 \operatorname{Pr}=100$ y $\lambda=0.005$ y $\mathrm{M}=2$

Cuadro 3.9: Polos para $\lambda=0.005$ y $\mathrm{M}=2$

\begin{tabular}{|c|c|c|c|}
\hline $\operatorname{Pr}=10$ & $\operatorname{Pr}=30$ & $\operatorname{Pr}=60$ & $\operatorname{Pr}=100$ \\
\hline-0.9597 & -1.0467 & -1.0683 & -1.0746 \\
\hline $0.3345+0.9379 \mathrm{i}$ & $0.3057+0.9783 \mathrm{i}$ & $0.2984+0.9868 \mathrm{i}$ & $0.2963+0.9892 \mathrm{i}$ \\
\hline $0.3345-0.9379 \mathrm{i}$ & $0.3057-0.9783 \mathrm{i}$ & $0.2984-0.9868 \mathrm{i}$ & $0.2963-0.9892 \mathrm{i}$ \\
\hline 0.7314 & 0.7282 & 0.7275 & 0.7274 \\
\hline
\end{tabular}


lo suficientemente altos. Este efecto puede compensarse con el uso del factor de peso. Cuando el valor de $\lambda$ alcanza el punto de inflexión de la Figura 3.9, valores altos de M y Pr no inestabilizan el sistema. Los Cuadros 3.10 y 3.11 y la Figura 3.13 muestran que cuando se alcanza un valor seguro de $\lambda$, unos valores inadecuados de M y Pr no causan la inestabilidad del sistema.

Cuadro 3.10: Polos para $\lambda=0.075, \operatorname{Pr}=100$ variando $\mathrm{M}$

\begin{tabular}{|c|c|c|c|}
\hline $\mathrm{M}=1$ & $\mathrm{M}=2$ & $\mathrm{M}=3$ & $\mathrm{M}=4$ \\
\hline-0.4760 & -0.5807 & -0.6109 & -0.5818 \\
\hline $0.2325+0.4221 \mathrm{i}$ & $0.4412+0.6557 \mathrm{i}$ & $0.4480+0.6961 \mathrm{i}$ & $0.4512+0.6643 \mathrm{i}$ \\
\hline $0.2325-0.4221 \mathrm{i}$ & $0.4412-0.6557 \mathrm{i}$ & $0.4480-0.6961 \mathrm{i}$ & $0.4512-0.6643 \mathrm{i}$ \\
\hline 0.9111 & 0.7638 & 0.7523 & 0.7579 \\
\hline
\end{tabular}

Cuadro 3.11: Polos para $\lambda=0.075, \mathrm{M}=2$ variando $\operatorname{Pr}$

\begin{tabular}{|c|c|c|c|}
\hline $\operatorname{Pr}=10$ & $\operatorname{Pr}=30$ & $\operatorname{Pr}=60$ & $\operatorname{Pr}=100$ \\
\hline-0.5104 & -0.5507 & -0.5732 & -0.5807 \\
\hline $0.4405+0.5737 \mathrm{i}$ & $0.4428+0.6221 \mathrm{i}$ & $0.4418+0.6474 \mathrm{i}$ & $0.4412+0.6557 \mathrm{i}$ \\
\hline $0.4405-0.5737 \mathrm{i}$ & $0.4428-0.6221 \mathrm{i}$ & $0.4418-0.6474 \mathrm{i}$ & $0.4412-0.6557 \mathrm{i}$ \\
\hline 0.7841 & 0.7711 & 0.7655 & 0.7638 \\
\hline
\end{tabular}

\subsection{Reglas de diseño preliminares}

Considerando lo explicado sobre la posición deseada de los polos en lazo cerrado y los resultados de las simulaciones, se pueden obtener varias conclusiones útiles:

- La respuesta temporal muestra oscilaciones limitadas cuando T disminuye y Pr es lo suficientemente alto.

- La respuesta temporal varía con Pr solo hasta un cierto valor, en el que alcanza un comportamiento de lazo abierto. Sin embargo, esto puede corregirse con el valor adecuado de $\mathrm{M}$.

- $\mathrm{n}_{\mathrm{g}}$ deja de tener influencia una vez se alcanza cierto valor.

- $\lambda$ funciona de forma opuesta a Pr, ya que ajusta el DMC haciendo que los polos complejos sean dominantes, pero mitigando la parte compleja para eliminar oscilaciones.

- Valores altos de Pr y M junto a valores demasiado bajos de $\lambda$ llevan el sistema a la inestabilidad.

Por lo tanto, se pueden deducir las siguientes reglas de diseño: 

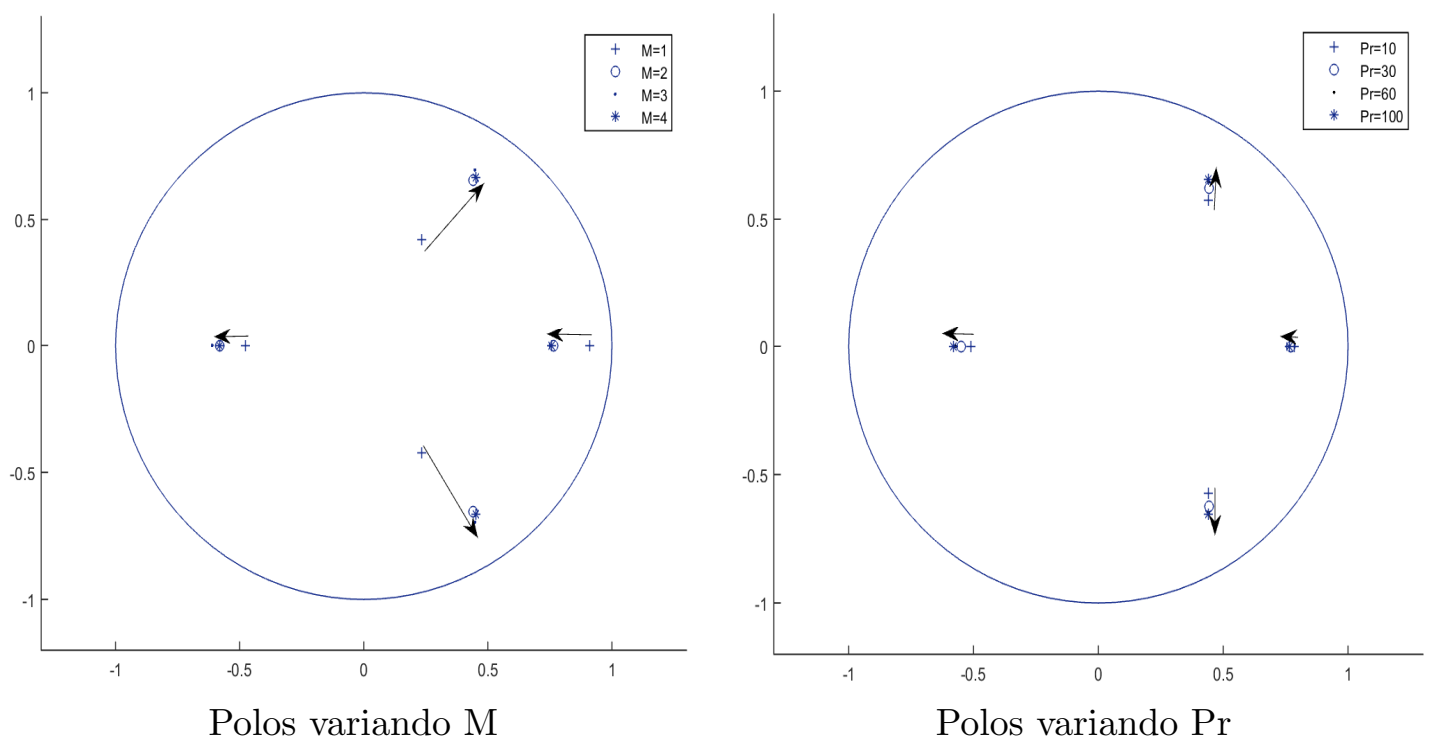

Figura 3.13: Polos para $\lambda=0.075$

1. $\mathrm{n}_{\mathrm{g}}=\operatorname{Pr}+\mathrm{n}$, donde $\mathrm{n}$ es el número requerido de valores g necesarios para alcanzar el el escalón unitario.

2. $\operatorname{Pr}=\tau_{\mathrm{p}}$, siendo $\tau_{\mathrm{p}}$ la constante de tiempo del equivalente FOPDT (tiempo para alcanzar el $63 \%$ del valor del escalón).

3. M tiene una influencia limitada en el proceso para valores pequeños de $\operatorname{Pr}$ (inferiores a $\tau_{\mathrm{p}}$ ) pero corrige el comportamiento como lazo abierto cuando Pr es demasiado alto (mayor a $\tau_{\mathrm{p}}$ ). También, según Rawlings y Muske [51], este valor debería ser mayor o igual al número de modos inestables del sistema para garantizar la estabilidad .

4. Para calcular el tiempo de muestreo se han tenido en cuenta las consideraciones de Shridhar y Cooper [16], que proponen tomar el valor mínimo entre $0,1 \tau_{\mathrm{p}}$ y $0,5 \theta$. Esto ha demostrado dar resultados precisos. Este valor se considerará un máximo.

5. $\lambda$ contribuye a suavizar las oscilaciones en la respuesta. Sin embargo, se ha observado que este efecto desaparece cuando el horizonte de predicción toma valores lo suficientemente altos. El motivo es la función de coste que se debe minimizar para calcular los los incrementos de control (Ecuación 2.21). Este función tiene dos términos. El primero de ellos (que depende del horizonte de predicción) es el error cuadrático medio y el segundo (que depende del horizonte de control) son los incrementos de control . La influencia de $\lambda$ se limita al segundo término que depende del horizonte de control, que suele tomar valores más bajos que el horizonte de predicción (la solución al DMC se da en la Ecuación 4.4. Este hecho implica(tal y como se hace notar en [52]) que una mala elección del resto de parámetros, limita la utilidad del factor de peso. 
6. $\lambda$ no debe tomar un valor más elevado que el punto de inflexión de la curva de primer incremento de control. Valores más altos no influirán significativamente en la respuesta temporal.

7. $\lambda$ también tiene un importante efecto previniendo la inestabilidad del sistema y debe tomar un valor adecuado: No más del punto de inflexión de la curva de primer incremento de control.

\subsection{Validación del método}

Para validar las conclusiones obtenidas en las secciones anteriores, estas se usarán para ajustar el control por DMC de los siguientes benchmarks (extraídos de [16]), pero sin las simplificaciones hechas anteriormente:

\subsubsection{Bechmarks}

En esta sección se presentan los bechmarks que se usarán para validar las reglas de diseño presentadas en la sección 3.5. Los Bechnmark de 1 a 4 han sido extraídos de [16].

Bechnmark 1

$$
\mathrm{G}(\mathrm{s})=\frac{\mathrm{e}^{-50 \mathrm{~s}}}{(150 \mathrm{~s}+1)(25 \mathrm{~s}+1)}
$$

Bechnmark 2

$$
\mathrm{G}(\mathrm{s})=\frac{(1-50 \mathrm{~s}) \mathrm{e}^{-10 \mathrm{~s}}}{(100 \mathrm{~s}+1)^{2}}
$$

Bechnmark 3

$$
\mathrm{G}(\mathrm{s})=\frac{(1+50 \mathrm{~s}) \mathrm{e}^{-10 \mathrm{~s}}}{(100 \mathrm{~s}+1)^{2}}
$$

Bechnmark 4

$$
G(s)=\frac{e^{-10 s}}{(50 s+1)^{4}}
$$

Bechnmark 5, este proceso ha sido extraído de [53], section 4.

$$
\mathrm{G}(\mathrm{s})=\frac{0.0039 \mathrm{~s}+0.0038}{\mathrm{~s}^{2}+0.075 \mathrm{~s}+0.0003}
$$

Aparte de por su función de transferencia, cada benchmark está caracterizado por su respuesta a un escalón unidad ( necesario para obtener la constante de tiempo y el el tiempo de establecimiento) y un gráfico que muestre la relación entre $\lambda$ y el primer $\Delta \mathrm{u}$, que permite obtener el rango correcto de valores para $\lambda$. El Cuadro 3.14 relaciona cada benchmark con su correspondiente curva. 


\begin{tabular}{|c|c|c|c|c|c|c|}
\hline Benchmark & Constante de tiempo & Tiempo de establecimiento & $\tau$ & $\mathrm{Pr}$ & $\mathrm{M}$ & $\mathrm{n}_{\mathrm{g}}$ \\
\hline 1 & 160 & 1000 & 16 & 10 & 2 & 73 \\
\hline 2 & 180 & 1100 & 18 & 10 & 2 & 73 \\
\hline 3 & 180 & 800 & 18 & 10 & 2 & 100 \\
\hline 4 & 220 & 500 & 22 & 10 & 2 & 33 \\
\hline 5 & 250 & 1100 & 25 & 10 & 2 & 54 \\
\hline
\end{tabular}

Cuadro 3.12: Resultados del ajuste para cada benchmark

\subsubsection{Resultados}

A partir de los datos presentados en la sección anterior y las reglas de diseño de la sección 3.5, se pueden calcular los parámetros del DMC para cada benchmark. Estos resultados se presentan en el Cuadro 3.12.

Según las conclusiones anteriores, los controladores deberán ajustarse usando un horizonte de predicción inferior a 10 (teniendo en cuenta que el tiempo de muestreo es $0,1 \tau_{\mathrm{p}}$ ). Para seleccionar un factor de peso adecuado, se usará la curva con los primeros incrementos de control. Como puede verse en la Figura 3.14 (usando el benchmark 1 como ejemplo), el valor del primer incremento de control cambia muy lentamente una vez se alcanza un valor de $\lambda=0.25$. Este es máximo valor útil, valores más altos solo harán más lento el proceso. Para $\lambda=0$ el primer incremento de control alcanza un valor de 6.5. Considerando que la ganancia del sistema y la referencia son 1, esto llevaría el sistema a la inestabilidad. La primera parte de la curva va de cero hasta el punto de inflexión de la misma. En esta parte, $\lambda$ tiene una fuerte influencia en la respuesta temporal. Una vez se sobrepasa el punto de inflexión, el factor de peso tiene una influencia pequeña. Este punto de inflexión se convierte en el máximo valor útil.

Las Figura 3.14 y 4.9 muestra la respuesta del sistema para tres valores de $\lambda$ : Un valor pequeño, el punto de inflexión de la curva y un valor superior al punto de inflexión. Los valores inferiores al punto de inflexión pueden originar respuestas con demasiadas oscilaciones, mientras que los valores superiores dan tiempos de establecimiento demasiado largos. También puede verse como las primeras 4 curvas de $\lambda$ son diferentes de la última. El motivo es que los cuatro primeros benchmarks tienen una ganancia de 1 mientras que la ganancia del benchmark 5 es aproximadamente de 10. Esto significa que el principal factor que influye en la forma de la curva es la ganancia del sistema. La ganancia del sistema es el factor a tener en cuenta a la hora de calcular $\lambda$. Esto está de acuerdo con el método de Shridhar y Cooper, que calcula el factor de peso a partir de la ganancia del sistema [16].

Como es lógico pensar, las Figuras 3.14 y 4.9 también muestran que cuanto mayor es la ganancia del sistema, mayor debe ser $\lambda$.

\subsection{Comparación con otros métodos}

En esta sección se comparará el método desarrollado en este capítulo con dos métodos extraídos de la literatura y mencionados en la sección 5.1: El método de Shridhar-Cooper 


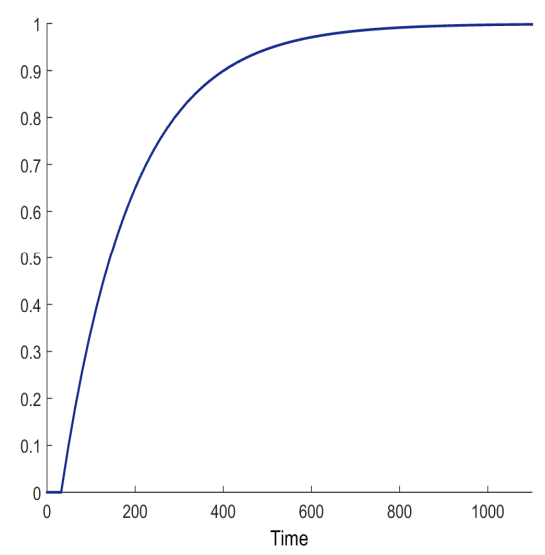

(a)(benchmark 1)

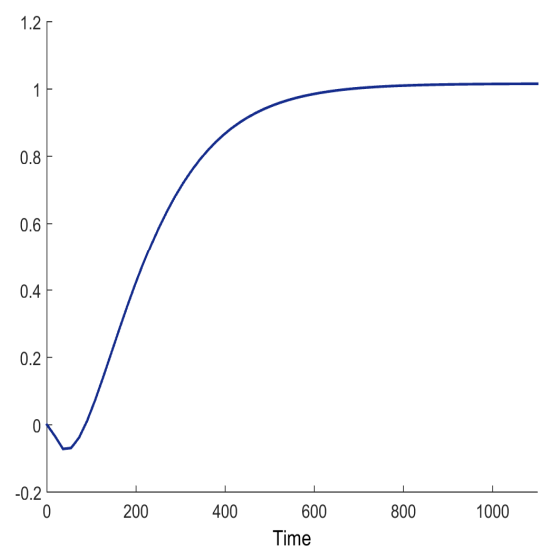

(a)(benchmark 2)

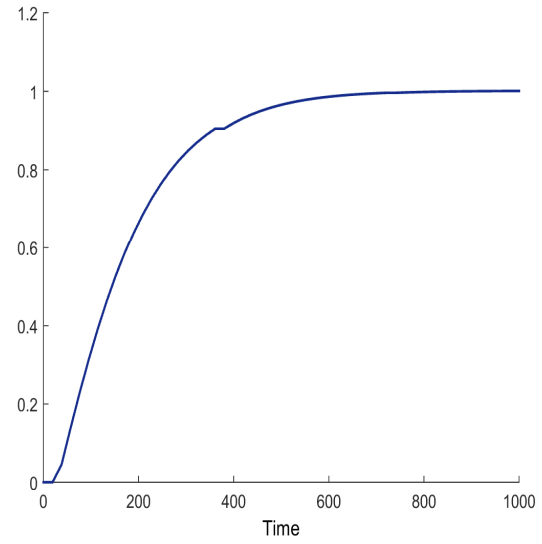

(a)(benchmark 3)

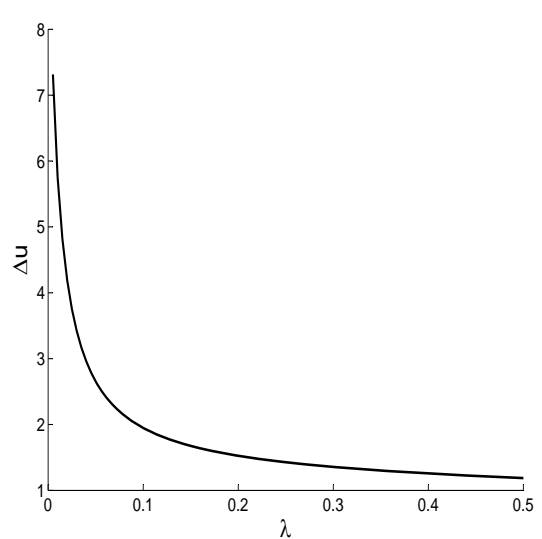

(b)(benchmark 1)

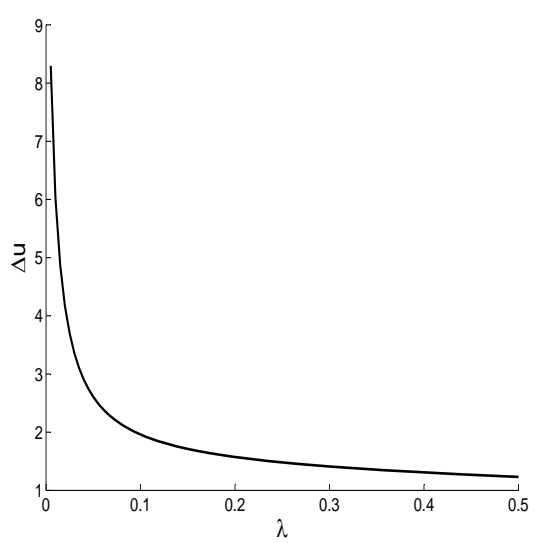

(b)(benchmark 2)

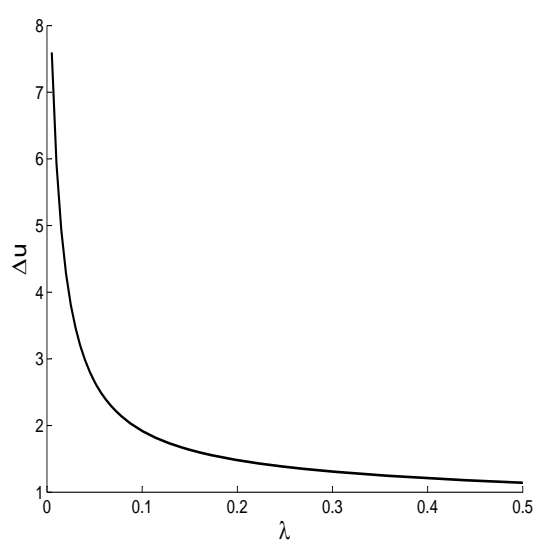

(b)(benchmark 3)
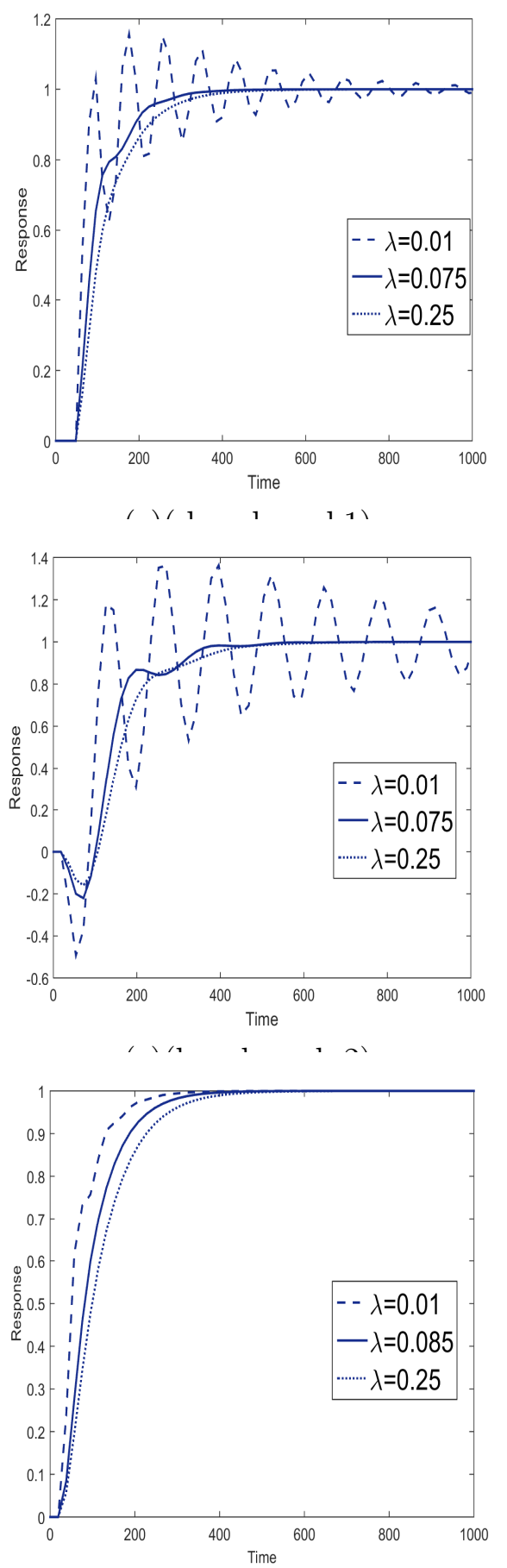

(c)(benchmark 3)

Figura 3.14: (a) Respuesta a escalón, (b) Curva de factor de peso (c) Respuesta temporal para cada benchmark dependiendo del valor de $\lambda$ 


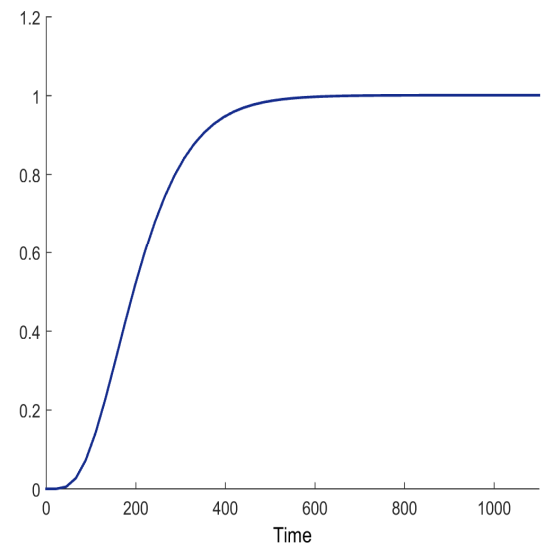

(a)(benchmark 4)

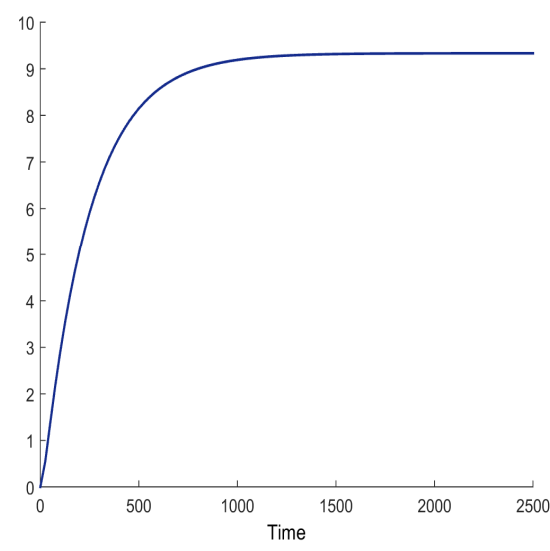

(a)(benchmark 5)

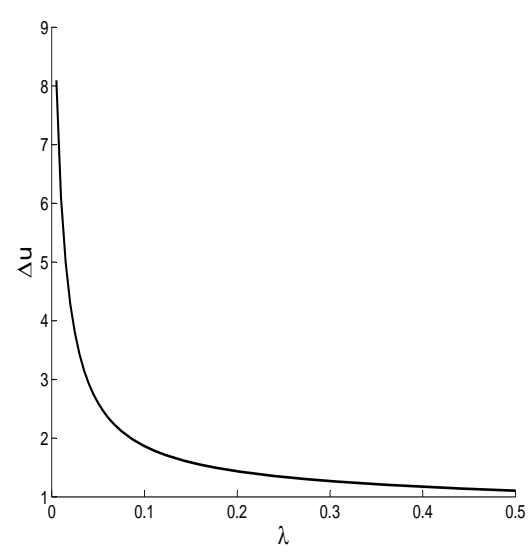

(b)(benchmark 4)

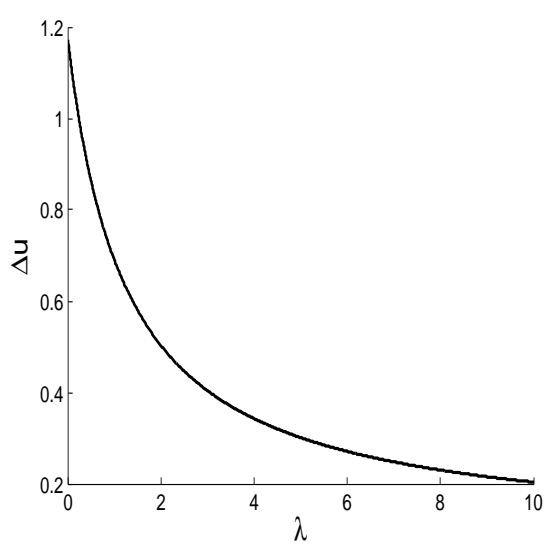

(b)(benchmark 5)

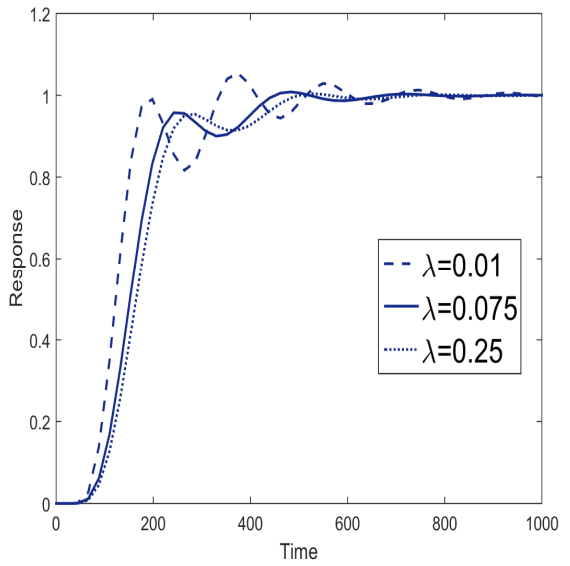

(c)(benchmark 4)

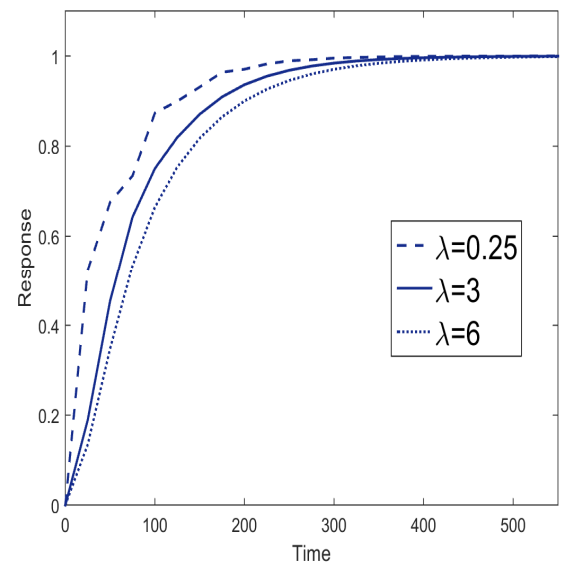

(c)(benchmark 5)

Figura 3.15: (a) Respuesta a escalón, (b) Curva de factor de peso (c) Respuesta temporal Respuesta temporal cada benchmark dependiendo del valor de $\lambda$ 
[16] y el método de Iglesias et al [18].

El método de Shridhar-Cooper se compone de los siguientes pasos:

1. Aproximar el sistema por un FOPDT.

$$
\frac{\mathrm{y}(\mathrm{s})}{\mathrm{u}(\mathrm{s})}=\frac{\mathrm{K}_{\mathrm{p}} \mathrm{e}^{\Theta \mathrm{p} s}}{\tau_{\mathrm{p}} \mathrm{s}+1}
$$

2. Seleccionar, si es posible, un tiempo de muestreo, $T$, que cumpla $\left(\tau_{\mathrm{p}}\right.$ es la constante de tiempo del FOPDT y $\Theta_{\mathrm{p}}$ el retraso del sistema):

$$
\mathrm{T} \leq 0,1 \tau_{\mathrm{p}} ; \mathrm{T} \leq 0,5 \Theta_{\mathrm{p}}
$$

3. Calcular el retraso discreto.

$$
\mathrm{k}=\frac{\Theta_{\mathrm{p}}}{\mathrm{T}}+1
$$

4. Calcular los horizontes de predicción y de modelo.

$$
\operatorname{Pr}=\mathrm{n}_{\mathrm{g}}=5 \frac{\tau_{\mathrm{p}}}{\mathrm{T}}+\mathrm{k}
$$

5. Seleccionar el horizonte de control y el factor de peso.

$$
\mathrm{f}=0 ; \mathrm{M}=1 \mathrm{f}=\frac{\mathrm{M}}{500}\left(\frac{3,5 \tau_{\mathrm{p}}}{\mathrm{T}}+2-\frac{\mathrm{M}-1}{2}\right) ; \mathrm{M} \geq 2 \lambda=\mathrm{fK}_{\mathrm{p}}^{2}
$$

Iglesias et al proponen una ecuación basada en métodos estadísticos que calcula el factor de peso. El resto de parámetros debe calcularse usando otro método. En este caso, se usará el método de Shridhar-Cooper.

$$
\lambda=1,631 \mathrm{~K}_{\mathrm{p}}\left(\frac{\Theta}{\tau}\right)^{0,4094}
$$

Donde $K_{p}$ es la ganacia del sistema, $\Theta$ es el retraso y $\tau$ la constante de tiempo del FOPDT.

El Cuadro 3.13 recoge los parámetros calculados para cada método. La Figura 3.16 compara la respuesta temporal de los tres métodos. El Cuadro 3.14 indica el error cuadrático medio de cada método con respecto a la referencia (un escalón unidad). Los resultados obtenidos demuestran que las reglas de sintonía propuestas en este trabajo consiguen una prestaciones superiores a las de métodos existentes.

\subsection{Prueba en una maqueta térmica}

La prueba final para las reglas de diseño desarrolladas será una prueba en un sistema real: una maqueta térmica con dos entradas y una salida. La maqueta se compone básicamente de (ver Figura 3.17): 


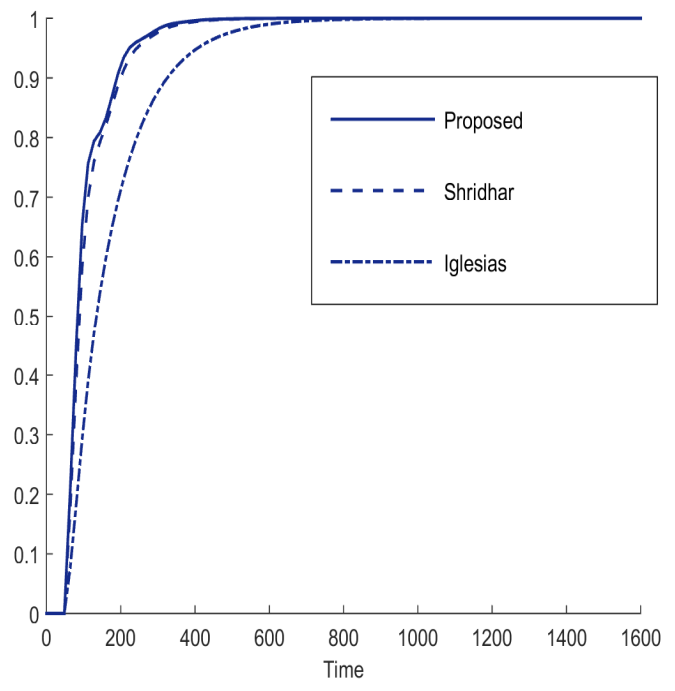

Comparación benchmark 1

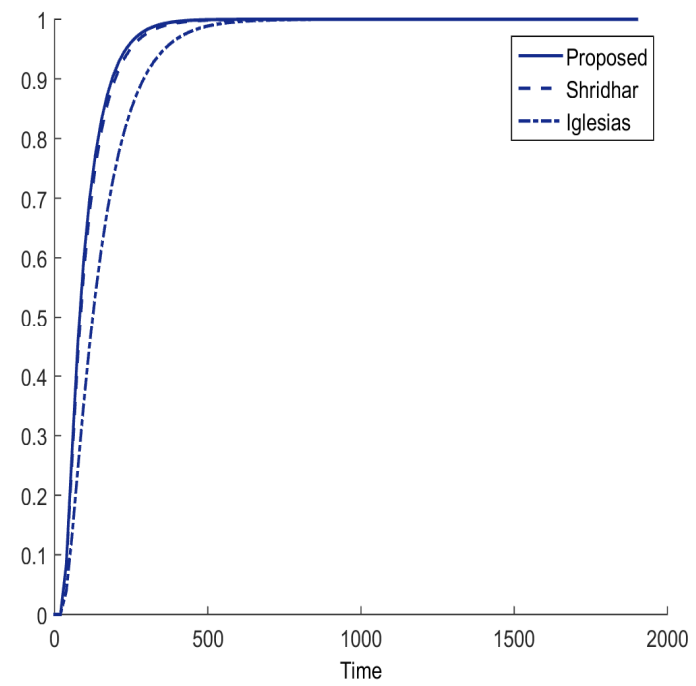

Comparación benchmark 3

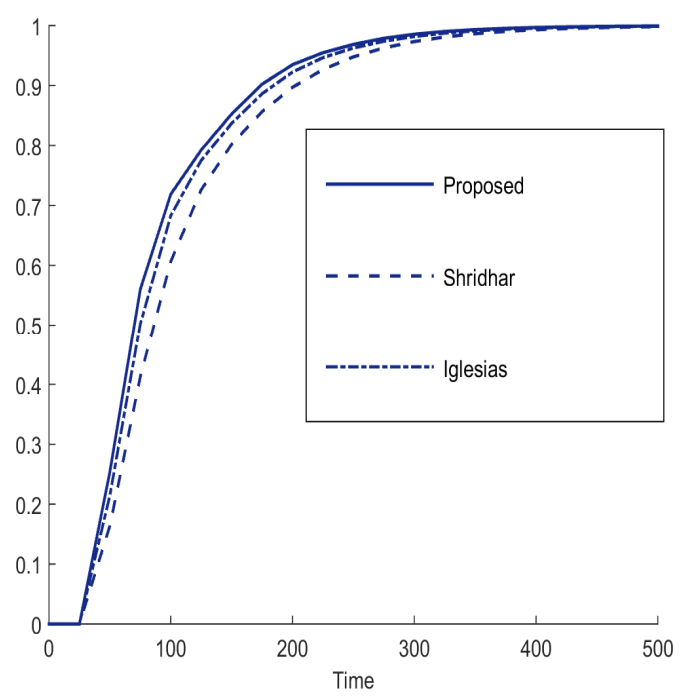

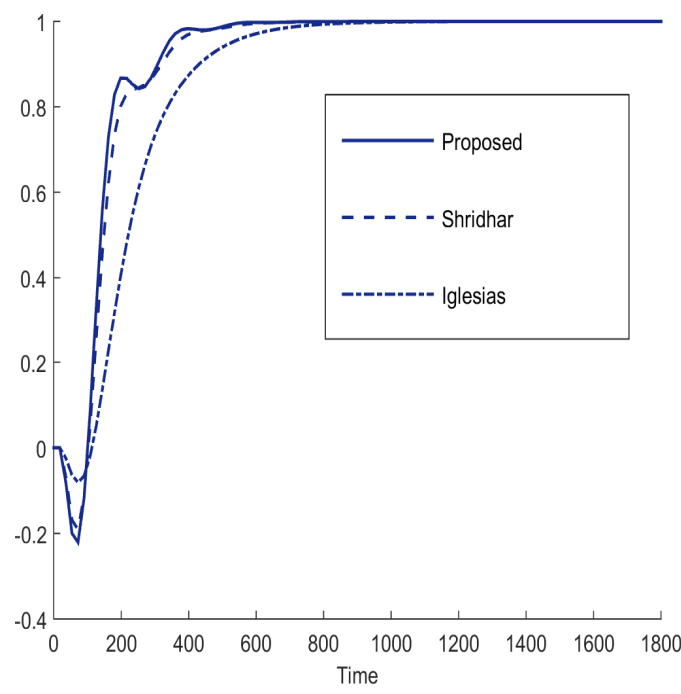

Comparación benchmark 2

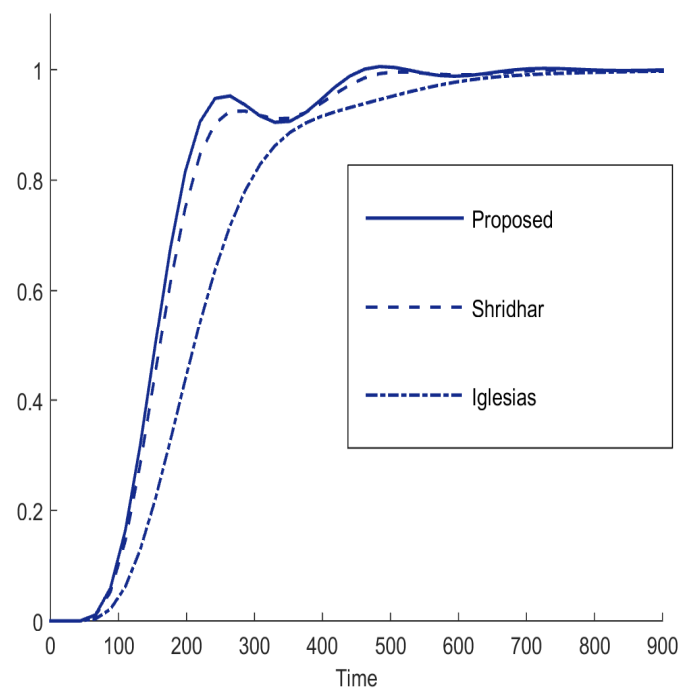

Comparación benchmark 4

Comparación benchmark 5

Figura 3.16: Comparación de resultados de los distintos métodos de ajuste en los benchmark propuestos 


\begin{tabular}{|c|c|c|c|c|c|c|c|c|c|c|}
\hline Benchmark & $\tau$ & Tiempo de establecimiento & $\mathrm{T}$ & $\mathrm{Pr}$ & $\mathrm{M}$ & $\mathrm{n}_{\mathrm{g}}$ & $\lambda$ & $\mathrm{K}_{\mathrm{p}}$ \\
\hline \multicolumn{7}{|c|}{ Parámetros según el método propuesto } \\
\hline 1 & 160 & 1000 & 16 & 10 & 2 & 73 & 0.075 & 1 \\
\hline 2 & 180 & 1100 & 18 & 10 & 2 & 73 & 0.075 & 1 \\
\hline 3 & 180 & 800 & 18 & 10 & 2 & 100 & 0.085 & 1 \\
\hline 4 & 220 & 500 & 22 & 10 & 2 & 33 & 0.075 & 1 \\
\hline 5 & 250 & 1100 & 25 & 10 & 2 & 53 & 3 & 9 \\
\hline \multicolumn{7}{|c|}{ Parámetros según el método de Shridhar-Cooper } \\
\hline 1 & 160 & 1000 & 16 & 53 & 2 & 53 & 0.146 & 1 \\
\hline 2 & 180 & 1100 & 18 & 57 & 2 & 57 & 0.146 & 1 \\
\hline 3 & 180 & 800 & 18 & 52 & 2 & 52 & 0.146 & 1 \\
\hline 4 & 220 & 500 & 22 & 56 & 2 & 56 & 0.146 & 1 \\
\hline 5 & 250 & 1100 & 53 & 2 & 53 & 11.83 & 9 \\
\hline \multicolumn{7}{|c|}{ Parámetros según el método de Iglesias et al } \\
\hline 1 & 160 & 1000 & 16 & 53 & 2 & 53 & 0.84 & 1 \\
\hline 2 & 180 & 1100 & 18 & 57 & 2 & 57 & 1.3 & 1 \\
\hline 3 & 180 & 800 & 22 & 52 & 2 & 52 & 0.63 & 1 \\
\hline 4 & 220 & 500 & 25 & 53 & 2 & 56 & 1.18 & 1 \\
\hline 5 & 250 & 1100 & 53 & 7.59 & 9 \\
\hline
\end{tabular}

Cuadro 3.13: Parámetros según los distintos métodos para cada uno de los benchmark

\begin{tabular}{|c|c|c|c|}
\hline Benchmark & Método propuesto & Método Shridhar Cooper & Método de Iglesias et al \\
\hline 1 & 0,0875 & 0,0917 & 0,1053 \\
\hline 2 & 0,1531 & 0,1568 & 0,1705 \\
\hline 3 & 0,1035 & 0,1076 & 0,1283 \\
\hline 4 & 0,0639 & 0,0664 & 0,0756 \\
\hline 5 & 0,0295 & 0,0382 & 0,0748 \\
\hline
\end{tabular}

Cuadro 3.14: Error cuadrático medio para cada método

- Un ventilador para bajar la temperatura.

- Un conjunto de resistencias (bombillas) que incrementan la temperatura.

- Un Pt100 para medir la temperatura.

En este sistema (Figura 3.18) la temperatura en el interior de la maqueta (salida) se controla mediante la tensión del ventilador y resistencias (entradas).

En la Figura 3.19 puede verse la respuesta a escalón del sistema. Las constantes de tiempo para el ventilador y la resistencia son:

- 76 segundos para la resistencia.

- 64 segundos para el ventilador. 


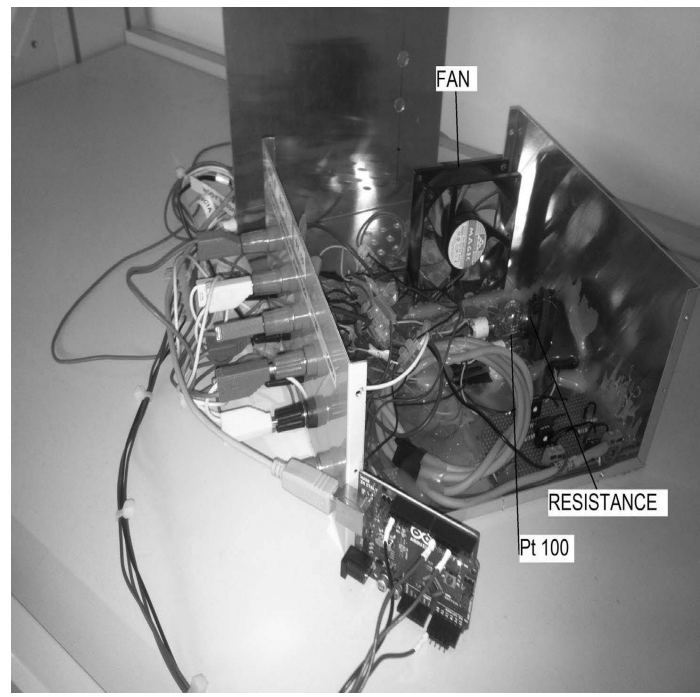

Maqueta térmica

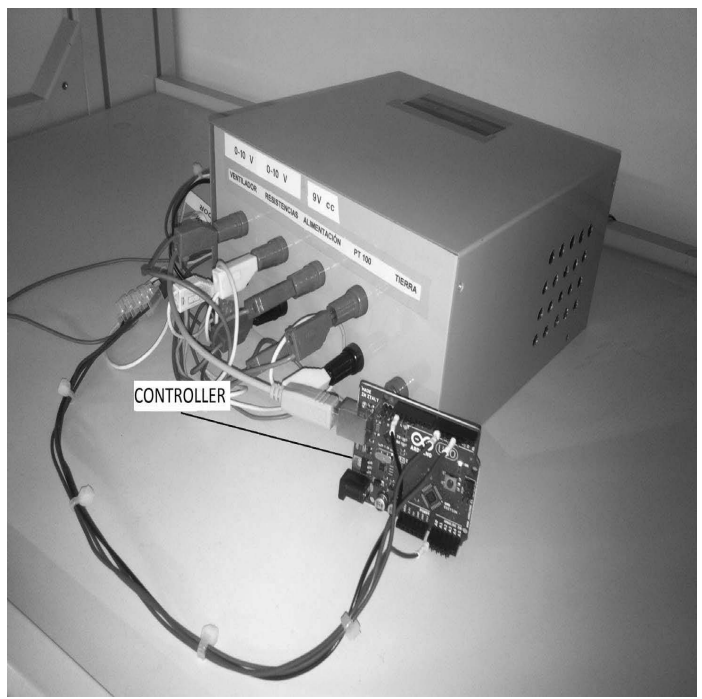

Interior maqueta térmica

Figura 3.17: Maqueta térmica

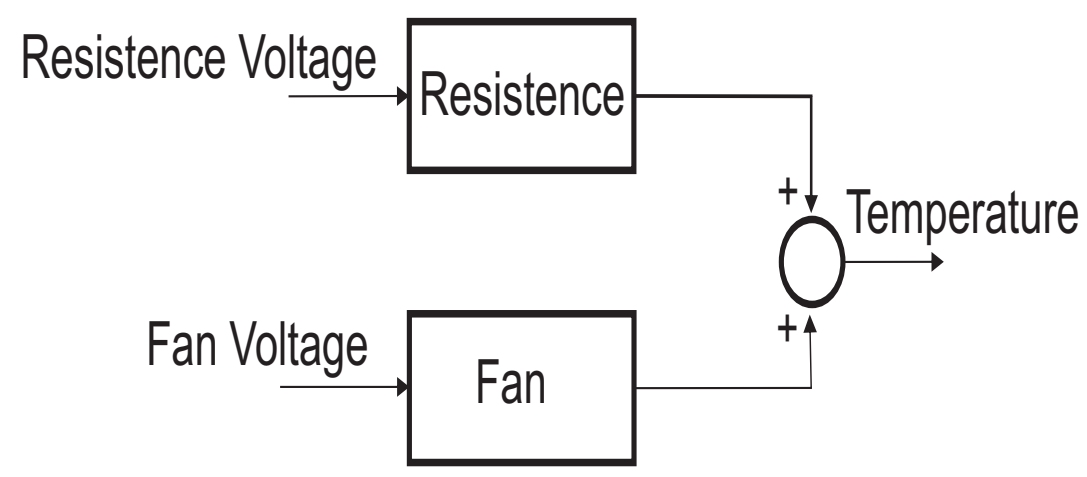

Figura 3.18: Diagrama bloques maqueta térmica 

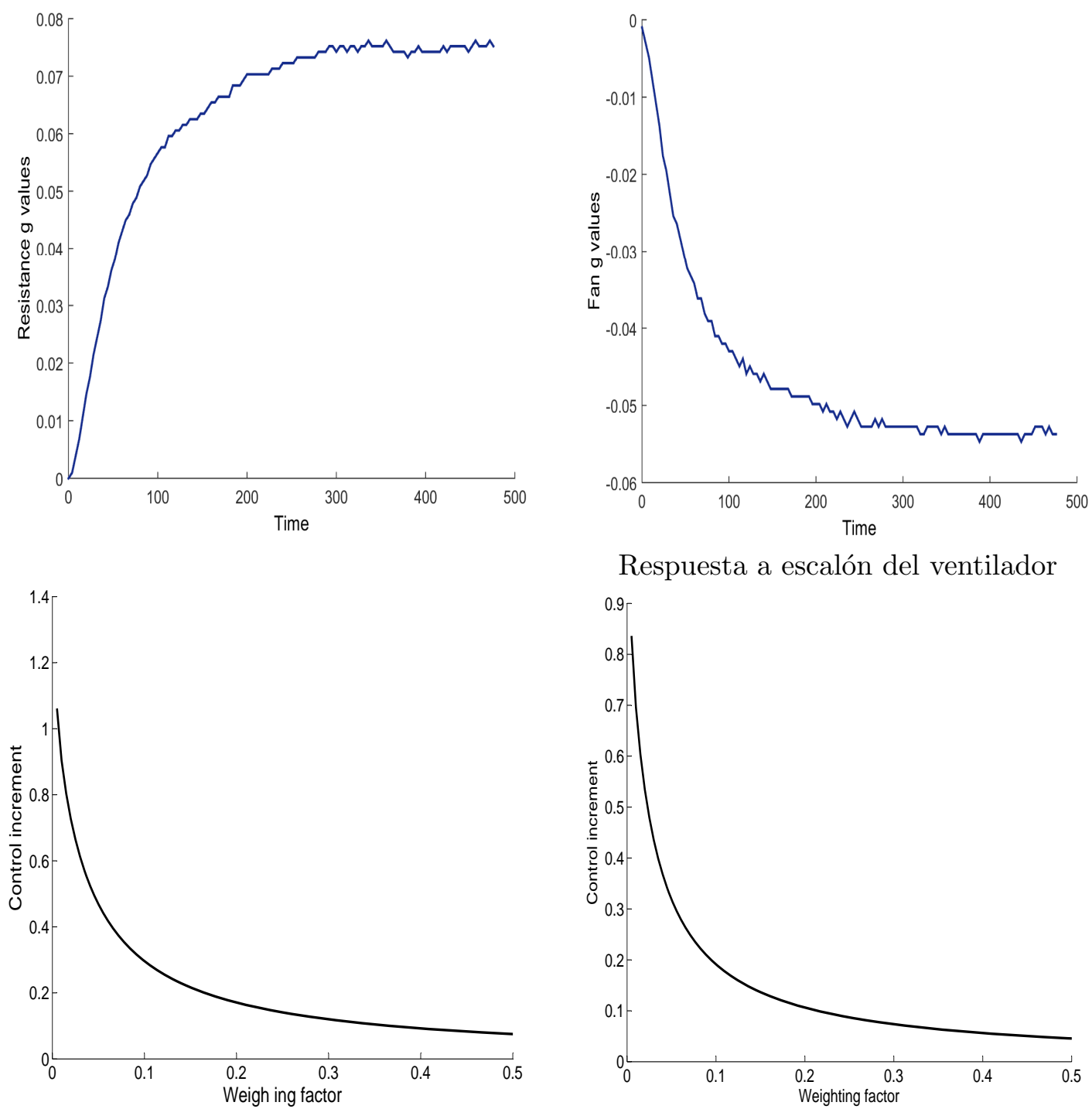

Respuesta a escalón del ventilador

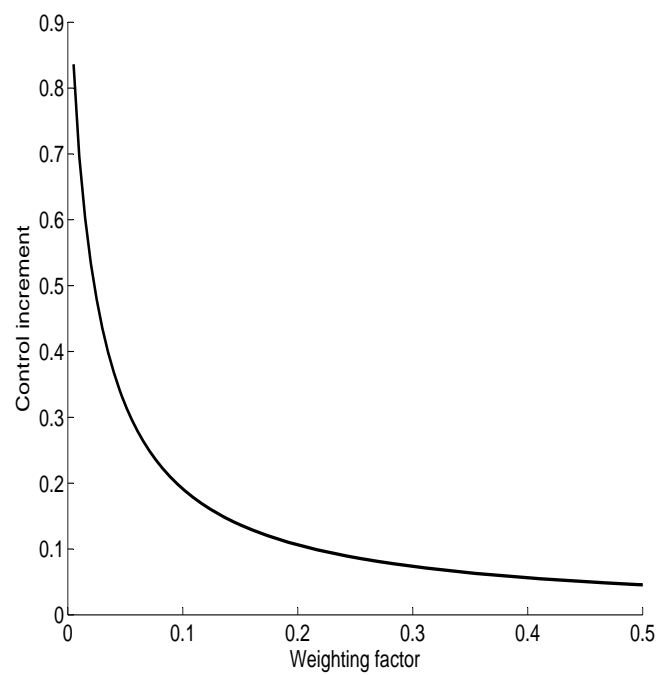

Curva de factor de peso de la resistencia

Curva de factor de peso del ventilador

Figura 3.19: Curvas de definición de la maqueta térmica 
Teniendo en cuenta que el tiempo de muestreo es de 4 segundos (impuesto por el sistema), el horizonte de predicción es 20. Para facilitar el estudio, se tomará el mismo horizonte de predicción para ambas entradas. El horizonte de modelo será 100, según la sección 3.4.4. Para el horizonte de control se fijará un valor pequeño, 2. Para seleccionar el factor de peso se deben trazar dos curvas con los primeros incrementos de control y $\lambda$ (Figura 3.19). Ambas curvas muestran que en esta maqueta $\lambda$ solo es útil hasta 0.2. Valores más altos darán incrementos de control muy pequeños y, consecuentemente, un control más lento. Además, valores demasiado altos de $\lambda$ harían que el sistema no pudiera manejar la inercia térmica.

\begin{tabular}{|c|c|c|c|c|}
\hline Caso & $\lambda$ & $\mathrm{n}_{\mathrm{g}}$ & $\mathrm{Pr}$ & $\mathrm{M}$ \\
\hline 1 & 0.1 & 101 & 20 & 2 \\
\hline 2 & 0.2 & 101 & 20 & 2 \\
\hline 3 & 0.01 & 101 & 20 & 2 \\
\hline 4 & 0.1 & 101 & 20 & 1 \\
\hline 5 & 0.1 & 101 & 20 & 5 \\
\hline 6 & 0.1 & 101 & 10 & 2 \\
\hline
\end{tabular}

Cuadro 3.15: Resultados maqueta térmica

La explicación de todos los casos es la siguiente (ver Cuadro 3.15 y Figura 3.20).

- Caso 1: Aquí se muestra el control obtenido aplicando las reglas de diseño propuestas. Un valor adecuado de $\lambda$ da más capacidad al ventilador y resistencia de tratar con la inercia térmica permitiendo mayores incrementos de control. Desde el punto de vista de la sección 3.4.3, este valor de $\lambda$ debilita el efecto de los polos complejos.

- Caso 2: Se muestra la consecuencia de un valor demasiado elevado de $\lambda$. Tal y como se explica en la sección 3.4.3, este valor ha hecho dominante a los polos complejos, resultando en en sistema con oscilaciones. Este valor inadecuado enlentece al ventilador y la resistencia haciendo que sea más difícil vencer la inercia térmica.

- Caso 3: Esta respuesta muestra el efecto de un valor mínimo de $\lambda$. En este proceso, solo significa un pequeño sobre pico.

- Caso 4: La diferencia entre esta caso y el 2 es difícil de ver. Solo se diferencian en el valor de M, y este parámetro solo muestra una influencia importante cuando Pr alcanza valores elevados.

- Caso 5: Puede verse el efecto de un valor demasiado alto de M. En la sección 3.4.2 se explica la influencia de los polos complejos con M y $\lambda$.

- Caso 6: Esta respuesta muestra el efecto de un Pr demasiado pequeño. El algoritmo DMC no tiene suficientes datos para llevar a cabo predicciones precisas. En la sección 3.4.2 se explica que un valor demasiado bajo para Pr hace que los polos complejos se vuelvan dominantes. 


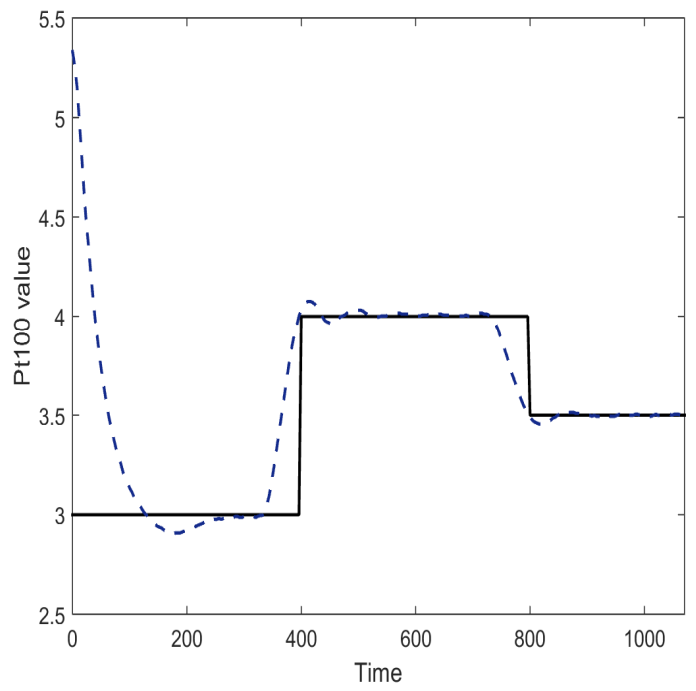

Caso 1

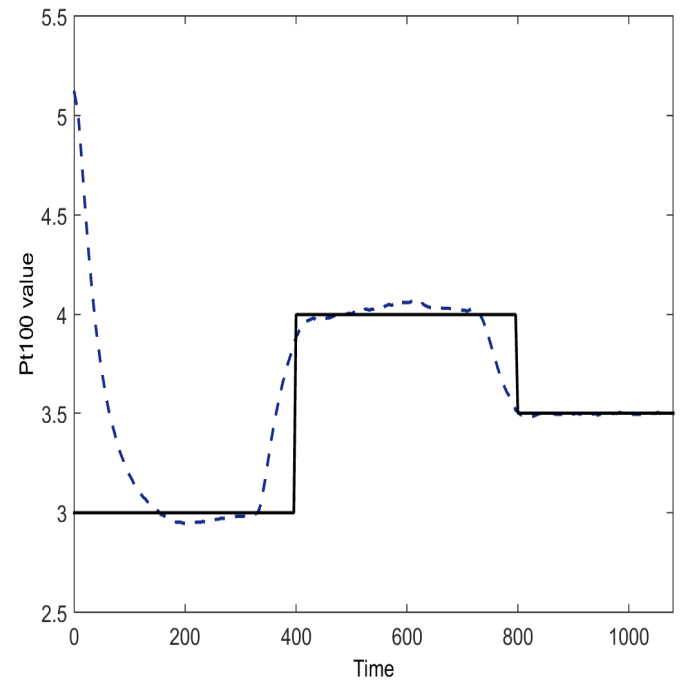

Caso 3

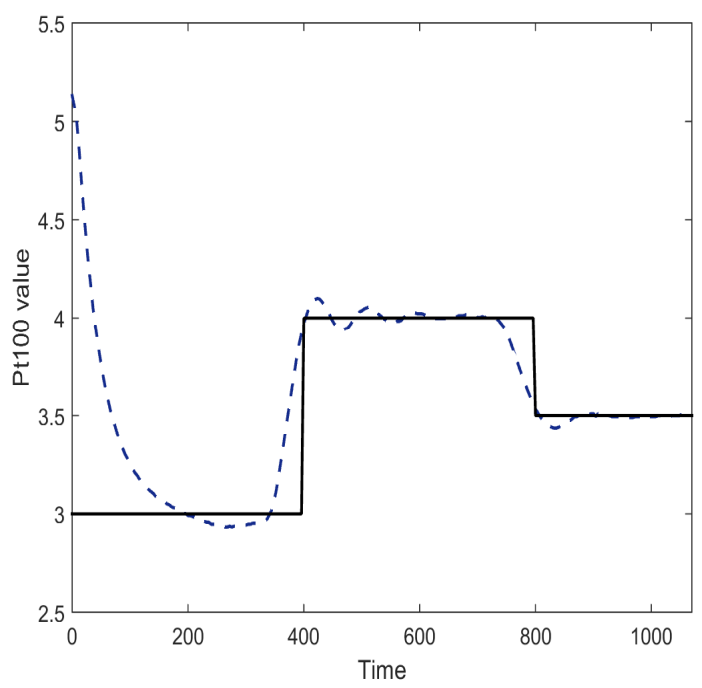

Caso 5

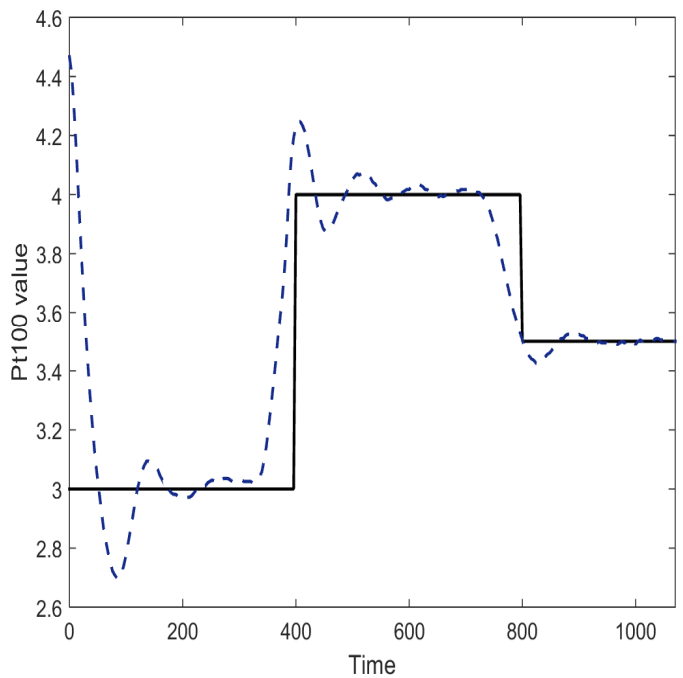

Caso 2

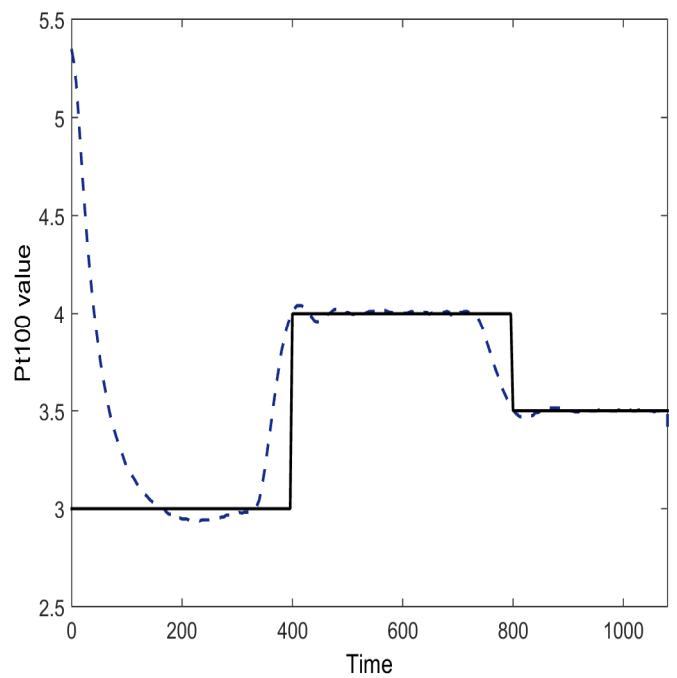

Caso 4

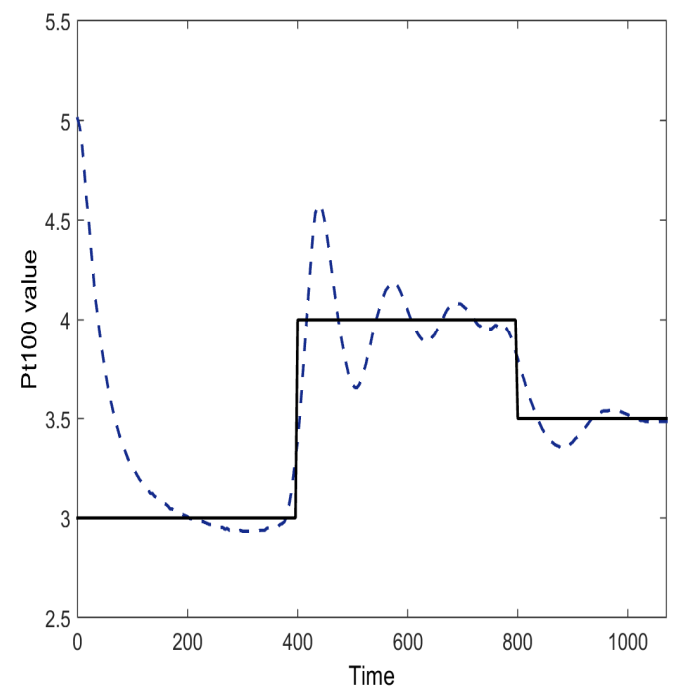

Caso 6

Figura 3.20: Resultados maqueta térmica. La línea continua indica la referencia y la discontinua la respuesta del sistema 


\subsection{Conclusiones}

En este capítulo se han desarrollado varias reglas de diseño para el DMC. Para cumplir este objetivo, se ha realizado un estudio de los parámetros del DMC en la respuesta temporal. El sistema controlado se transforma en un sistema Lineal Invariante en el Tiempo para hallar los polos que determinan la dinámica del sistema. Se usa una aproximación de primer orden con retraso para reducir el número de polos y hacer más fácil el análisis. El movimiento de los polos en lazo cerrado se traduce en valores para el horizonte de predicción, de control, de modelo y el factor de peso. Esto permite obtener varias reglas para un arranque rápido.

La aplicación de esta guía no proporciona una solución óptima, pero permite un control aceptable y los parámetros obtenidos se pueden usar como punto de partida para ajustes posteriores. Se presentan los resultados de este métodos sobre una serie de benchmarks en simulación y una planta real para demostrar que se han cumplido los objetivos. También se hace una comparación con otros métodos de ajuste. 


\section{Capítulo 4}

\section{Formulación LTI para sistemas MIMO}

\subsection{Introducción}

En la sección anterior se han descrito los pasos para expresar el algoritmo DMC, para el caso monovariable, mediante una formulación LTI, que posibilite usar técnicas de análisis de sistemas. Esto ha permitido obtener y validar una serie de reglas de diseño que posibiliten un ajuste inicial con un rendimiento aceptable, aunque no óptimo.

Sin embargo, la gran mayoría de procesos industriales son multivariable, es decir, tienen varias entradas y varias salidas. Muchos de ellos se controlan siguiendo una estrategia monovariable, implementando tantos lazos de control como variables controladas (tal y como se muestra en la Figura 4.2). Esta estrategia es válida siempre y cuando las interacciones entre lazos de control $\left(\mathrm{G}_{12}\right.$ and $\left.\mathrm{G}_{21}\right)$ sean despreciables o asumibles.

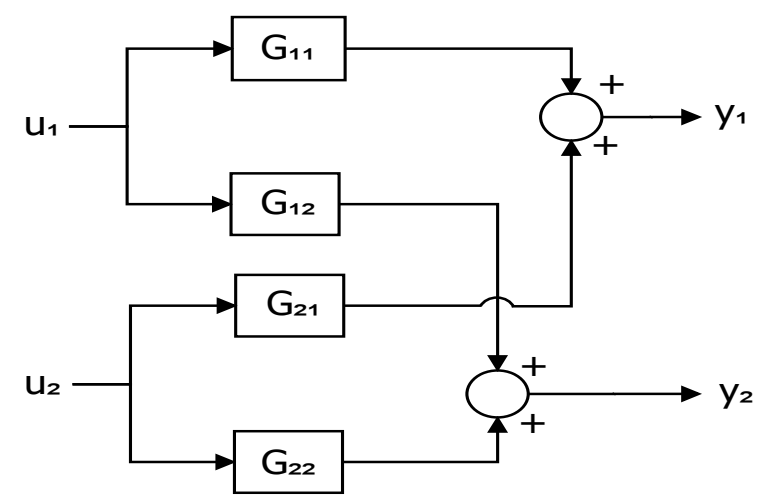

Figura 4.1: Representación en funciones de transferencia de un sistema MIMO lineal 2x2

En los casos en los que no se puede recurrir a esta estrategia, es necesario recurrir a un método de control centralizado que tenga en cuenta todos los lazos de control y sus 


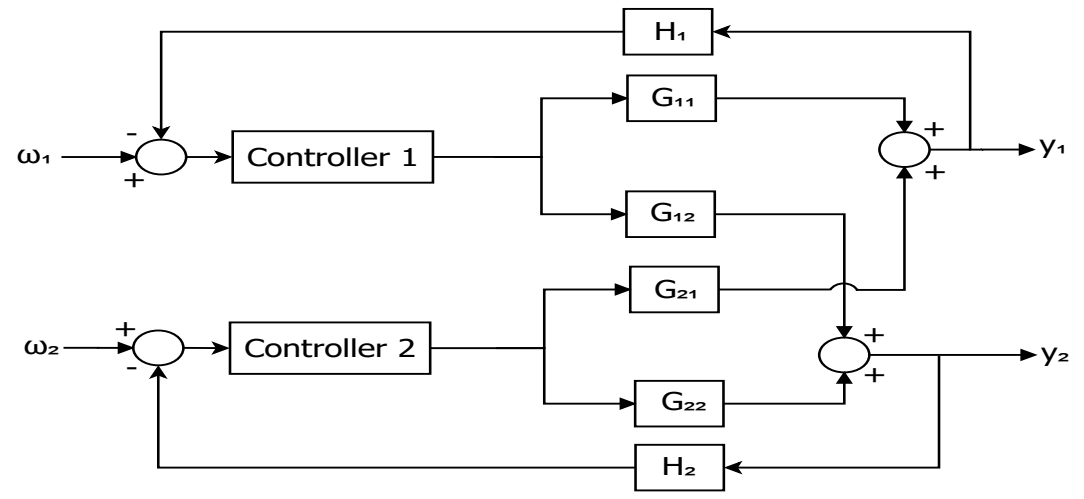

Figura 4.2: Sistema MIMO 2x2 controlado usando lazos de control SISO

interacciones, como el control predictivo, a pesar de ser más complicadas de implementar.

El objetivo de este capítulo es obtener una formulación LTI para procesos multivariables controlados con DMC para, en un capítulo posterior, usar el estudio de los polos para obtener reglas de diseño para sistemas MIMO.

Este capítulo se estructura como sigue:

- Section 4.2. Esta sección desarrollará la formulación LTI y la función de transferencia para sistemas MIMO controlados por DMC, centrándose en el cas TITO.

- Section 4.3. Esta sección expandirá los resultados de la sección anterior para sistemas MIMO [z x z].

- Section 4.4. En esta sección se validarán la formulación LTI mediante la simulación de varios benchmark encontrados en la literatura.

- Section 4.5. En esta sección se usará la formulación para demostrar el efecto desacoplador del DMC, como ejemplo de aplicación.

\subsection{Desarrollo del DMC como un sistema LTI MIMO para el caso $[2 \times 2]$}

En la Figura 4.3 puede verse una planta típica [2x2] controlada mediante DMC.

En esta sección, dicha planta [2x2] se expresará con una formulación LTI (Figura 4.4 y ecuaciones 4.1, 4.2 and 4.3), que emplea notación matricial y considera el ruido y las perturbaciones iguales a cero. Este desarrollo se dividirá en dos pasos:

- En el primer paso, se usará la solución del DMC como punto de partida para obtener el diagrama de bloques LTI.

- En el segundo paso, dicho diagrama de bloques se simplificará usando notación matricial y se calculará la matriz de transferencia. 


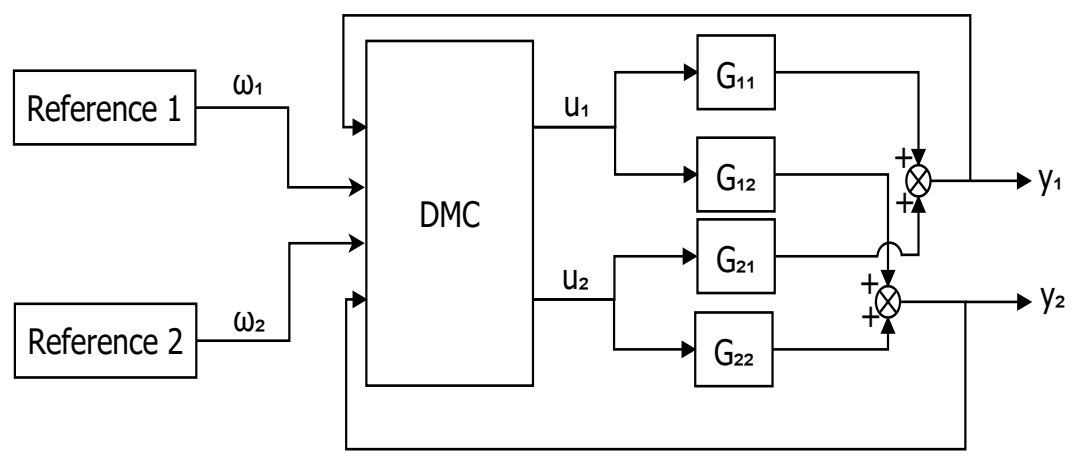

Figura 4.3: Sistema controlado por DMC

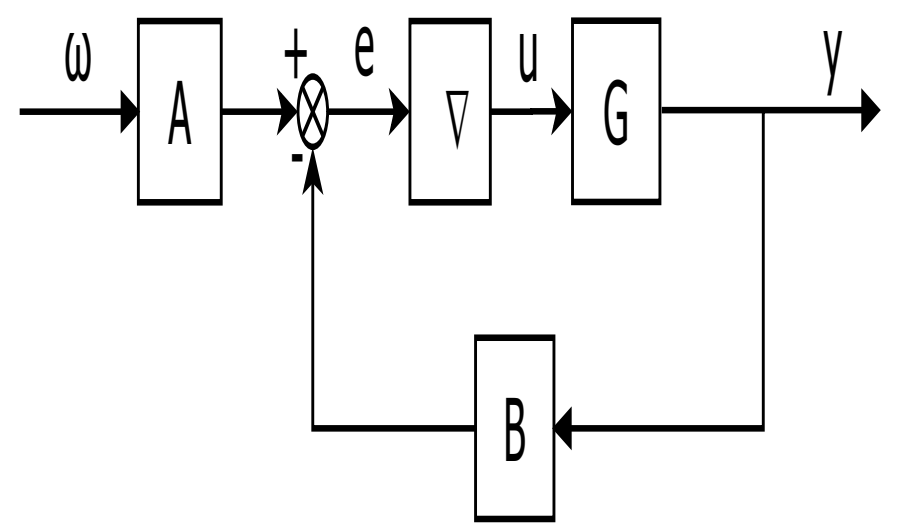

Figura 4.4: Representación típica MIMO LTI usando notación matricial 


$$
\begin{gathered}
\mathrm{y}=\mathrm{Gu} \\
\mathrm{u}=\nabla \mathrm{e} \\
\mathrm{e}=\omega \mathrm{A}-\mathrm{By}
\end{gathered}
$$

\subsubsection{Diagrama de bloques LTI}

Tal y como se ha explicado antes, en esta sección se usará la solución del DMC como punto de partida para obtener las ecuaciones básicas de la formulación LTI y el diagrama de bloques.

La solución del DMC se puede expresar como Ecuación 4.4.

$$
\begin{aligned}
& \boldsymbol{\Delta} \mathbf{u}(\mathbf{t})=\left(\mathbf{J}^{\mathbf{T}} \mathbf{J}+\lambda \mathbf{I}\right)^{-\mathbf{1}} \mathbf{J}^{\mathbf{T}}(\boldsymbol{\omega}-\mathbf{P})
\end{aligned}
$$

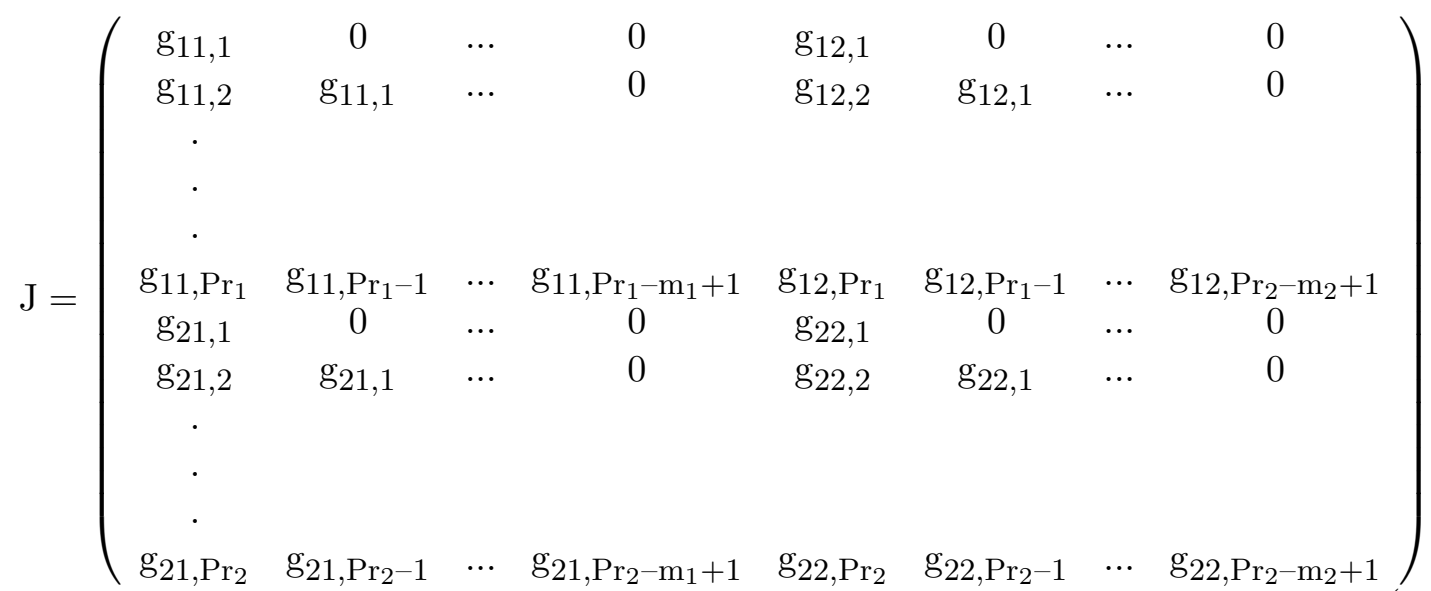

Donde $\omega$ es la referencia, $\mathrm{J}$ la matriz dinámica del sistema construida con los coeficientes de respuesta a salto $\left(\mathrm{g}_{\mathrm{ij}}\right), \operatorname{Pr}_{1}$ y $\operatorname{Pr}_{2}$ son los horizontes de predicción, $\mathrm{m}_{1}$ and $\mathrm{m}_{2}$ son los horizontes de control,$\lambda$ es el factor de peso, $\mathbf{u}$ es la señal de control y $\mathbf{P}$ es la respuesta libre ( la parte de la respuesta que no depende de las acciones de control futuras).

La Ecuación 4.4 se puede simplificar en la Ecuación 4.8 usando la Ecuación 4.6:

$$
\mathbf{\Phi}=\left(\mathbf{J}^{\mathrm{T}} \mathbf{J}+\lambda \mathbf{I}\right)^{-1} \mathbf{J}^{\mathrm{T}}
$$




$$
\Phi=\left(\begin{array}{ccccc}
\phi_{11} & \ldots & \phi_{1 \mathrm{Pr}_{1}} & \ldots & \phi_{1\left(\operatorname{Pr}_{1}+\mathrm{Pr}_{2}\right)} \\
\phi_{21} & \ldots & \phi_{2 \mathrm{Pr}_{1}} & \ldots & \phi_{2\left(\operatorname{Pr}_{1}+\mathrm{Pr}_{2}\right)} \\
\cdot & & & & \\
\cdot & & & & \\
\cdot & & & & \\
\phi_{\mathrm{m}_{1} 1} & \ldots & \phi_{\mathrm{m}_{1} \operatorname{Pr}_{1}} & \ldots & \phi_{\mathrm{m}_{1}\left(\operatorname{Pr}_{1}+\operatorname{Pr}_{2}\right)} \\
\phi_{\left(\mathrm{m}_{1}+1\right) 1} & \ldots & \phi_{\left(\mathrm{m}_{1}+1\right) \operatorname{Pr}_{1}} & \ldots & \phi_{\left(\mathrm{m}_{1}+1\right)\left(\operatorname{Pr}_{1}+\operatorname{Pr}_{2}\right)} \\
\cdot & & & & \\
\cdot & & & & \\
\phi_{\left(\mathrm{m}_{1}+\mathrm{m}_{2}\right) 1} & \ldots & \phi_{\left(\mathrm{m}_{1}+\mathrm{m}_{2}\right) \operatorname{Pr}_{1}} & \ldots & \phi\left(\mathrm{m}_{1}+\mathrm{m}_{2}\right)\left(\operatorname{Pr}_{1}+\operatorname{Pr}_{2}\right)
\end{array}\right)
$$

En control predictivo solo el primer elemento de cada variable manipulada de $\boldsymbol{\Delta} \mathbf{u}$ $\left(\Delta \mathrm{u}_{1}(\mathrm{t})\right.$ y $\left.\Delta \mathrm{u}_{2}(\mathrm{t})\right)$ se manda a la planta (debido a la estrategia del horizonte recesivo,[14]), no el vector completo. esto implica que solo dos columnas de $\Phi$ tienen realmente interés, son las que se usa para calcular $\Delta \mathrm{u}_{1}(\mathrm{t})$ y $\Delta \mathrm{u}_{2}(\mathrm{t})$. Por lo tanto, la Ecuación 4.8 puede simplificarse en la Ecuación 4.9, en la que solo se consideran las columnas útiles de $\Phi$ ("1" y " $\left.\mathrm{m}_{1}+1 "\right)$. Debe tenerse en cuenta que $\phi_{\left(\mathrm{m}_{1}+1\right) 1}, \phi_{\left(\mathrm{m}_{1}+1\right) 2}, \ldots, \phi_{\left(\mathrm{m}_{1}+1\right)\left(\operatorname{Pr}_{1}+\operatorname{Pr}_{2}\right)}$ se ha renombrado a $\phi_{21}, \phi_{22}, \ldots, \phi_{2\left(\operatorname{Pr}_{1}+\operatorname{Pr}_{2}\right)}$ por simplicidad.

$\left(\begin{array}{c}\Delta \mathrm{u}_{1}(\mathrm{t}) \\ \Delta \mathrm{u}_{2}(\mathrm{t})\end{array}\right)=\left(\begin{array}{cccc}\phi_{11} & \phi_{12} & \ldots & \phi_{1\left(\operatorname{Pr}_{1}+\mathrm{Pr}_{2}\right)} \\ \phi_{21} & \phi_{22} & \ldots & \phi_{2\left(\operatorname{Pr}_{1}+\mathrm{Pr}_{2}\right)}\end{array}\right)\left(\begin{array}{c}\omega_{1}(\mathrm{t}+1) \\ \cdot \\ \cdot \\ \cdot \\ \omega_{1}\left(\mathrm{t}+\mathrm{Pr}_{1}\right) \\ \omega_{2}(\mathrm{t}+1) \\ \cdot \\ \cdot \\ \cdot \\ \omega_{2}\left(\mathrm{t}+\mathrm{Pr}_{2}\right)\end{array}\right)-\left(\begin{array}{c}\mathrm{P}_{1}(\mathrm{t}+1) \\ \cdot \\ \cdot \\ \cdot \\ \mathrm{P}_{1}\left(\mathrm{t}+\mathrm{Pr}_{1}\right) \\ \mathrm{P}_{2}(\mathrm{t}+1) \\ \cdot \\ \cdot \\ \cdot \\ \mathrm{P}_{2}\left(\mathrm{t}+\operatorname{Pr}_{2}\right)\end{array}\right)$ 
Desarrollando este producto de matrices, se obtienen $\Delta \mathrm{u}_{1}(\mathrm{t})$ y $\Delta \mathrm{u}_{2}(\mathrm{t})$ (Ecuaciones 4.10 y 4.11$)$.

$$
\begin{aligned}
& \Delta \mathrm{u}_{1}(\mathrm{t})=\left[\left(\sum_{\mathrm{i}=1}^{\operatorname{Pr}_{1}} \phi_{1 \mathrm{i}} \omega_{1}(\mathrm{t}+\mathrm{i})\right)+\left(\sum_{\mathrm{i}=\operatorname{Pr}_{1}+1}^{\operatorname{Pr}_{1}+\operatorname{Pr}_{2}} \phi_{1 \mathrm{i}} \omega_{2}\left(\mathrm{t}+\mathrm{i}-\mathrm{Pr}_{1}\right)\right)\right]- \\
& {\left[\left(\sum_{\mathrm{i}=1}^{\operatorname{Pr}_{1}} \phi_{1 \mathrm{i}} \mathrm{P}_{1}(\mathrm{t}+\mathrm{i})\right)-\left(\sum_{\mathrm{i}=\operatorname{Pr}_{1}+1}^{\operatorname{Pr}_{1}+\mathrm{Pr}_{2}} \phi_{1 \mathrm{i}} \mathrm{P}_{2}\left(\mathrm{t}+\mathrm{i}-\mathrm{Pr}_{1}\right)\right)\right]} \\
& \Delta \mathrm{u}_{2}(\mathrm{t})=\left[\left(\sum_{\mathrm{i}=1}^{\operatorname{Pr}_{1}} \phi_{2 \mathrm{i}} \omega_{1}(\mathrm{t}+\mathrm{i})\right)+\left(\sum_{\mathrm{i}=\operatorname{Pr}_{1}+1}^{\operatorname{Pr}_{1}+\operatorname{Pr}_{2}} \phi_{2 \mathrm{i}} \omega_{2}\left(\mathrm{t}+\mathrm{i}-\operatorname{Pr}_{1}\right)\right)\right]- \\
& {\left[\left(\sum_{\mathrm{i}=1}^{\operatorname{Pr}_{1}} \phi_{2 \mathrm{i}} \mathrm{P}_{1}(\mathrm{t}+\mathrm{i})\right)-\left(\sum_{\mathrm{i}=\operatorname{Pr}_{1}+1}^{\operatorname{Pr}_{1}+\operatorname{Pr}_{2}} \phi_{2 \mathrm{i}} \mathrm{P}_{2}\left(\mathrm{t}+\mathrm{i}-\operatorname{Pr}_{1}\right)\right)\right]}
\end{aligned}
$$

La respuesta libre se puede expresar como sigue [14] ( $\mathrm{N}$ es el horizonte de modelo, el número de coeficientes "g" utilizados para calcular la respuesta libre).

$$
\begin{gathered}
\mathrm{P}(\mathrm{t}+\mathrm{k})=\mathrm{y}_{\mathrm{m}}(\mathrm{t})+\sum_{\mathrm{i}=1}^{\mathrm{N}} \mathrm{s}_{\mathrm{i}}^{\mathrm{k}} \Delta \mathrm{u}(\mathrm{t}-\mathrm{i})=\mathrm{y}_{\mathrm{m}}(\mathrm{t})+\mathrm{S}_{\mathrm{n}}^{\mathrm{k}}\left(\mathrm{z}^{-1}\right) \Delta \mathrm{u} \\
\mathrm{s}_{\mathrm{n}}^{\mathrm{k}}=\mathrm{g}_{\mathrm{n}+\mathrm{k}}-\mathrm{g}_{\mathrm{n}} \\
\mathrm{S}_{\mathrm{n}}^{\mathrm{k}}\left(\mathrm{z}^{-1}\right)=\mathrm{s}_{1}^{\mathrm{k}} \mathrm{z}^{-1}+\mathrm{s}_{2}^{\mathrm{k}} \mathrm{z}^{-2}+\ldots+\mathrm{s}_{\mathrm{n}}^{\mathrm{k}} \mathrm{z}^{-\mathrm{n}}
\end{gathered}
$$

Para sistemas MIMO [2x2], las Ecuaciones 4.12, 4.13 y 4.14 se convierten en:

$$
\begin{gathered}
\mathrm{P}_{\mathrm{i}}(\mathrm{t}+\mathrm{k})=\mathrm{y}_{\mathrm{i}, \mathrm{m}}(\mathrm{t})+\sum_{\mathrm{j}=1}^{2}\left(\mathrm{~S}_{\mathrm{n}, \mathrm{ij}}^{\mathrm{k}}\left(\mathrm{z}^{-1}\right) \Delta \mathrm{u}_{\mathrm{j}}\right) \\
\mathrm{s}_{\mathrm{n}, \mathrm{ij}}^{\mathrm{k}}=\mathrm{g}_{\mathrm{n}+\mathrm{k}, \mathrm{ij}}-\mathrm{g}_{\mathrm{n}, \mathrm{ij}} \\
\mathrm{S}_{\mathrm{n}, \mathrm{ij}}^{\mathrm{k}}\left(\mathrm{q}^{-1}\right)=\mathrm{s}_{1, \mathrm{ij}}^{\mathrm{k}} \mathrm{z}^{-1}+\mathrm{s}_{2, \mathrm{ij}}^{\mathrm{k}} \mathrm{z}^{-2}+\ldots+\mathrm{s}_{\mathrm{n}, \mathrm{ij}}^{\mathrm{k}} \mathrm{z}^{-\mathrm{n}}
\end{gathered}
$$

Luego, las ecuaciones 4.10 y 4.11 se convierten en:

$$
\begin{aligned}
\Delta \mathrm{u}_{1}(\mathrm{t})= & {\left[\left(\sum_{\mathrm{i}=1}^{\operatorname{Pr}_{1}} \phi_{1 \mathrm{i}} \omega_{1}(\mathrm{t}+\mathrm{i})\right)+\left(\sum_{\mathrm{i}=\operatorname{Pr}_{1}+1}^{\operatorname{Pr}_{1}+\operatorname{Pr}_{2}} \phi_{1 \mathrm{i}} \omega_{2}\left(\mathrm{t}+\mathrm{i}-\operatorname{Pr}_{1}\right)\right)\right]-\left[\left(\sum _ { \mathrm { i } = 1 } ^ { \operatorname { P r } _ { 1 } } \phi _ { 1 \mathrm { i } } \left(\mathrm{y}_{1, \mathrm{~m}}(\mathrm{t})\right.\right.\right.} \\
& \left.\left.\left.+\sum_{\mathrm{j}=1}^{2}\left(\mathrm{~S}_{\mathrm{n}, 1 \mathrm{j}}^{\mathrm{i}}\left(\mathrm{z}^{-1}\right) \Delta \mathrm{u}_{\mathrm{j}}\right)\right)\right)+\left(\sum_{\mathrm{i}=\operatorname{Pr}_{1}+1}^{\operatorname{Pr}_{1}+\operatorname{Pr}_{2}} \phi_{1 \mathrm{i}}\left(\mathrm{y}_{2, \mathrm{~m}}(\mathrm{t})+\sum_{\mathrm{j}=1}^{2}\left(\mathrm{~S}_{\mathrm{n}, 2 \mathrm{j}}^{\mathrm{i}}\left(\mathrm{z}^{-1}\right) \Delta \mathrm{u}_{\mathrm{j}}\right)\right)\right)\right]
\end{aligned}
$$




$$
\begin{aligned}
\Delta \mathrm{u}_{2}(\mathrm{t})= & {\left[\left(\sum_{\mathrm{i}=1}^{\operatorname{Pr}_{1}} \phi_{2 \mathrm{i}} \omega_{1}(\mathrm{t}+\mathrm{i})\right)+\left(\sum_{\mathrm{i}=\operatorname{Pr}_{1}+1}^{\operatorname{Pr}_{1}+\operatorname{Pr}_{2}} \phi_{2 \mathrm{i}} \omega_{2}\left(\mathrm{t}+\mathrm{i}-\operatorname{Pr}_{1}\right)\right)\right]-\left[\left(\sum _ { \mathrm { i } = 1 } ^ { \operatorname { P r } _ { 1 } } \phi _ { 2 \mathrm { i } } \left(\mathrm{y}_{1, \mathrm{~m}}(\mathrm{t})+\right.\right.\right.} \\
& \left.\left.\left.\sum_{\mathrm{j}=1}^{2}\left(\mathrm{~S}_{\mathrm{n}, 1 \mathrm{j}}^{\mathrm{i}}\left(\mathrm{z}^{-1}\right) \Delta \mathrm{u}_{\mathrm{j}}\right)\right)\right)+\left(\sum_{\mathrm{i}=\operatorname{Pr}_{1}+1}^{\operatorname{Pr}_{1}+\operatorname{Pr}_{2}} \phi_{2 \mathrm{i}}\left(\mathrm{y}_{2, \mathrm{~m}}(\mathrm{t})+\sum_{\mathrm{j}=1}^{2}\left(\mathrm{~S}_{\mathrm{n}, 2 \mathrm{j}}^{\mathrm{i}}\left(\mathrm{z}^{-1}\right) \Delta \mathrm{u}_{\mathrm{j}}\right)\right)\right)\right]
\end{aligned}
$$

En este punto, por simplicidad, se implementarán las simplificaciones descritas en el Cuadro 4.1. Se asume un cambio en la referencia en forma de escalón $(\omega(\mathrm{t})=\omega(\mathrm{t}+1)=$ $\omega(\mathrm{t}+2)=\ldots=\omega(\mathrm{t}+\mathrm{k}))$. Esto hace que los términos A se vuelvan escalares, en lugar de

\begin{tabular}{|c|c|c|c|}
\hline $\mathrm{A}_{11}$ & $\mathrm{~A}_{12}$ & $\mathrm{~A}_{21}$ & $\mathrm{~A}_{22}$ \\
\hline$\sum_{\mathrm{j}=1}^{\operatorname{Pr}_{1}} \phi_{1 \mathrm{j}}$ & $\sum_{\mathrm{j}=\operatorname{Pr}_{1}+1}^{\operatorname{Pr}_{1}+\operatorname{Pr}_{2}} \phi_{1 \mathrm{j}}$ & $\sum_{\mathrm{j}=1}^{\operatorname{Pr}_{1}} \phi_{2 \mathrm{j}}$ & $\sum_{\mathrm{j}=\operatorname{Pr}_{1}+1}^{\mathrm{Pr}_{1}+\operatorname{Pr}_{2}} \phi_{2 \mathrm{j}}$ \\
\hline $\mathrm{B}_{11}$ & $\mathrm{~B}_{12}$ & $\mathrm{~B}_{21}$ & $\mathrm{~B}_{22}$ \\
\hline$\sum_{\mathrm{j}=1}^{\operatorname{Pr}_{1}} \phi_{1 \mathrm{j}}$ & $\sum_{\mathrm{j}=\operatorname{Pr}_{1}+1}^{\operatorname{Pr}_{1}+\operatorname{Pr}_{2}} \phi_{1 \mathrm{j}}$ & $\sum_{\mathrm{j}=1}^{\operatorname{Pr}_{1}} \phi_{2 \mathrm{j}}$ & $\sum_{\mathrm{j}=\operatorname{Pr}_{1}+1}^{\mathrm{Pr}_{1}+\operatorname{Pr}_{2}} \phi_{2 \mathrm{j}}$ \\
\hline & $\mathrm{C}_{1 \mathrm{i}}$ & & $\mathrm{C}_{2 \mathrm{i}}$ \\
\hline \multicolumn{2}{|c|}{$\sum_{\mathrm{j}=1}^{\mathrm{Pr}_{1}} \phi_{1 \mathrm{j}}\left(\left(\mathrm{S}_{\mathrm{n}, 1 \mathrm{j}}^{\mathrm{j}}\left(\mathrm{z}^{-1}\right)\right)\right.$} & \multicolumn{2}{|c|}{$\sum_{\mathrm{j}=\operatorname{Pr}_{1}+1}^{\operatorname{Pr}_{1}+\operatorname{Pr}_{2}} \phi_{1 \mathrm{j}}\left(\left(\mathrm{S}_{\mathrm{n}, 2 \mathrm{i}}^{\mathrm{j}-\operatorname{Pr}_{1}+1}\left(\mathrm{z}^{-1}\right)\right)\right.$} \\
\hline & $\mathrm{D}_{1 \mathrm{i}}$ & & $\mathrm{D}_{2 \mathrm{i}}$ \\
\hline \multicolumn{2}{|c|}{$\sum_{\mathrm{j}=1}^{\mathrm{Pr}_{1}} \phi_{2 \mathrm{j}}\left(\left(\mathrm{S}_{\mathrm{n}, 1 \mathrm{j}}^{\mathrm{j}}\left(\mathrm{z}^{-1}\right)\right)\right.$} & \multicolumn{2}{|c|}{$\sum_{\mathrm{j}=\mathrm{Pr}_{1}+1}^{\mathrm{Pr}_{1}+\mathrm{Pr}_{2}} \phi_{2 \mathrm{j}}\left(\left(\mathrm{S}_{\mathrm{n}, 2 \mathrm{i}}^{\mathrm{j}-\mathrm{Pr}_{1}+1}\left(\mathrm{z}^{-1}\right)\right)\right.$} \\
\hline
\end{tabular}
polinomios en $\mathrm{z}^{-1}$.

Cuadro 4.1: Simplificaciones

Teniendo en cuenta las simplificaciones del Cuadro 4.1, las Ecuaciones 4.18 y 4.19 se pueden expresar como:

$$
\begin{aligned}
\Delta \mathrm{u}_{1}(\mathrm{t})= & \mathrm{A}_{11} \omega_{1}(\mathrm{t})+\mathrm{A}_{12} \omega_{2}(\mathrm{t})-\mathrm{B}_{11} \mathrm{y}_{1}(\mathrm{t})-\mathrm{B}_{12} \mathrm{y}_{2}(\mathrm{t})-\mathrm{C}_{11}\left(\mathrm{z}^{-1}\right) \Delta \mathrm{u}_{1}(\mathrm{t}) \\
& -\mathrm{C}_{12}\left(\mathrm{z}^{-1}\right) \Delta \mathrm{u}_{2}(\mathrm{t})-\mathrm{C}_{21}\left(\mathrm{z}^{-1}\right) \Delta \mathrm{u}_{1}(\mathrm{t})-\mathrm{C}_{22}\left(\mathrm{z}^{-1}\right) \Delta \mathrm{u}_{2}(\mathrm{t}) \\
\Delta \mathrm{u}_{2}(\mathrm{t})= & \mathrm{A}_{21} \omega_{1}(\mathrm{t})+\mathrm{A}_{22} \omega_{2}(\mathrm{t})-\mathrm{B}_{21} \mathrm{y}_{1}(\mathrm{t})-\mathrm{B}_{22} \mathrm{y}_{2}(\mathrm{t})-\mathrm{D}_{11}\left(\mathrm{z}^{-1}\right) \Delta \mathrm{u}_{1}(\mathrm{t}) \\
& -\mathrm{D}_{12}\left(\mathrm{z}^{-1}\right) \Delta \mathrm{u}_{2}(\mathrm{t})-\mathrm{D}_{21}\left(\mathrm{z}^{-1}\right) \Delta \mathrm{u}_{1}(\mathrm{t})-\mathrm{D}_{22}\left(\mathrm{z}^{-1}\right) \Delta \mathrm{u}_{2}(\mathrm{t})
\end{aligned}
$$

Y realizando varias simplificaciones más: 


$$
\begin{aligned}
& \mathrm{C}_{1}\left(\mathrm{z}^{-1}\right)=\mathrm{C}_{11}\left(\mathrm{z}^{-1}\right)+\mathrm{C}_{12}\left(\mathrm{z}^{-1}\right) \\
& \mathrm{C}_{2}\left(\mathrm{z}^{-1}\right)=\mathrm{C}_{21}\left(\mathrm{z}^{-1}\right)+\mathrm{C}_{22}\left(\mathrm{z}^{-1}\right) \\
& \mathrm{D}_{1}\left(\mathrm{z}^{-1}\right)=\mathrm{D}_{11}\left(\mathrm{z}^{-1}\right)+\mathrm{D}_{12}\left(\mathrm{z}^{-1}\right) \\
& \mathrm{D}_{2}\left(\mathrm{z}^{-1}\right)=\mathrm{D}_{21}\left(\mathrm{z}^{-1}\right)+\mathrm{D}_{22}\left(\mathrm{z}^{-1}\right)
\end{aligned}
$$

Reemplazando $4.22,4.23,4.24$ and 4.25 en las ecuaciones 4.20 y 4.21 , se obtienen las siguientes expresiones:

$$
\begin{aligned}
\Delta \mathrm{u}_{1}(\mathrm{t})= & \mathrm{A}_{11} \omega_{1}(\mathrm{t})+\mathrm{A}_{12} \omega_{2}(\mathrm{t})-\mathrm{B}_{11} \mathrm{y}_{1}(\mathrm{t})-\mathrm{B}_{12} \mathrm{y}_{2}(\mathrm{t}) \\
- & \mathrm{C}_{1}\left(\mathrm{z}^{-1}\right) \Delta \mathrm{u}_{1}(\mathrm{t})-\mathrm{C}_{2}\left(\mathrm{z}^{-1}\right) \Delta \mathrm{u}_{2}(\mathrm{t}) \\
\Delta \mathrm{u}_{2}(\mathrm{t})= & \mathrm{A}_{21} \omega_{1}(\mathrm{t})+\mathrm{A}_{22} \omega_{2}(\mathrm{t})-\mathrm{B}_{21} \mathrm{y}_{1}(\mathrm{t})-\mathrm{B}_{22} \mathrm{y}_{2}(\mathrm{t}) \\
& -\mathrm{D}_{1}\left(\mathrm{z}^{-1}\right) \Delta \mathrm{u}_{1}(\mathrm{t})-\mathrm{D}_{2}\left(\mathrm{z}^{-1}\right) \Delta \mathrm{u}_{2}(\mathrm{t})
\end{aligned}
$$

Las Ecuaciones 4.26 y 4.27 pueden seguir desarrollándose:

$$
\begin{aligned}
\Delta \mathrm{u}_{1}(\mathrm{t})\left(1+\mathrm{C}_{1}\left(\mathrm{z}^{-1}\right)\right)= & \mathrm{A}_{11} \omega_{1}(\mathrm{t})+\mathrm{A}_{12} \omega_{2}(\mathrm{t})-\mathrm{B}_{11} \mathrm{y}_{1}(\mathrm{t}) \\
& -\mathrm{B}_{12} \mathrm{y}_{2}(\mathrm{t})-\mathrm{C}_{2}\left(\mathrm{z}^{-1}\right) \Delta \mathrm{u}_{2}(\mathrm{t}) \\
\Delta \mathrm{u}_{2}(\mathrm{t})\left(1+\mathrm{D}_{2}\left(\mathrm{z}^{-1}\right)\right)= & \mathrm{A}_{21} \omega_{1}(\mathrm{t})+\mathrm{A}_{22} \omega_{2}(\mathrm{t})-\mathrm{B}_{21} \mathrm{y}_{1}(\mathrm{t}) \\
& -\mathrm{B}_{22} \mathrm{y}_{2}(\mathrm{t})-\mathrm{D}_{1}\left(\mathrm{z}^{-1}\right) \Delta \mathrm{u}_{1}(\mathrm{t})
\end{aligned}
$$

Las Ecuaciones 4.28 y 4.29 describen el sistema mostrado en la Figura 4.5.

\subsubsection{Matriz de transferencia LTI}

En esta sección, se simplificarán los diagramas de bloques de la Figura 4.5 y se calculará la matriz del sistema. Usando notación matricial para bloques y variables:

$$
\begin{aligned}
& \mathbf{A}=\left(\begin{array}{ll}
A_{11} & A_{12} \\
A_{21} & A_{22}
\end{array}\right) \\
& \mathbf{B}=\left(\begin{array}{ll}
B_{11} & B_{12} \\
B_{21} & B_{22}
\end{array}\right) \\
& \mathbf{G}=\left(\begin{array}{ll}
G_{11} & G_{12} \\
G_{21} & G_{22}
\end{array}\right)
\end{aligned}
$$




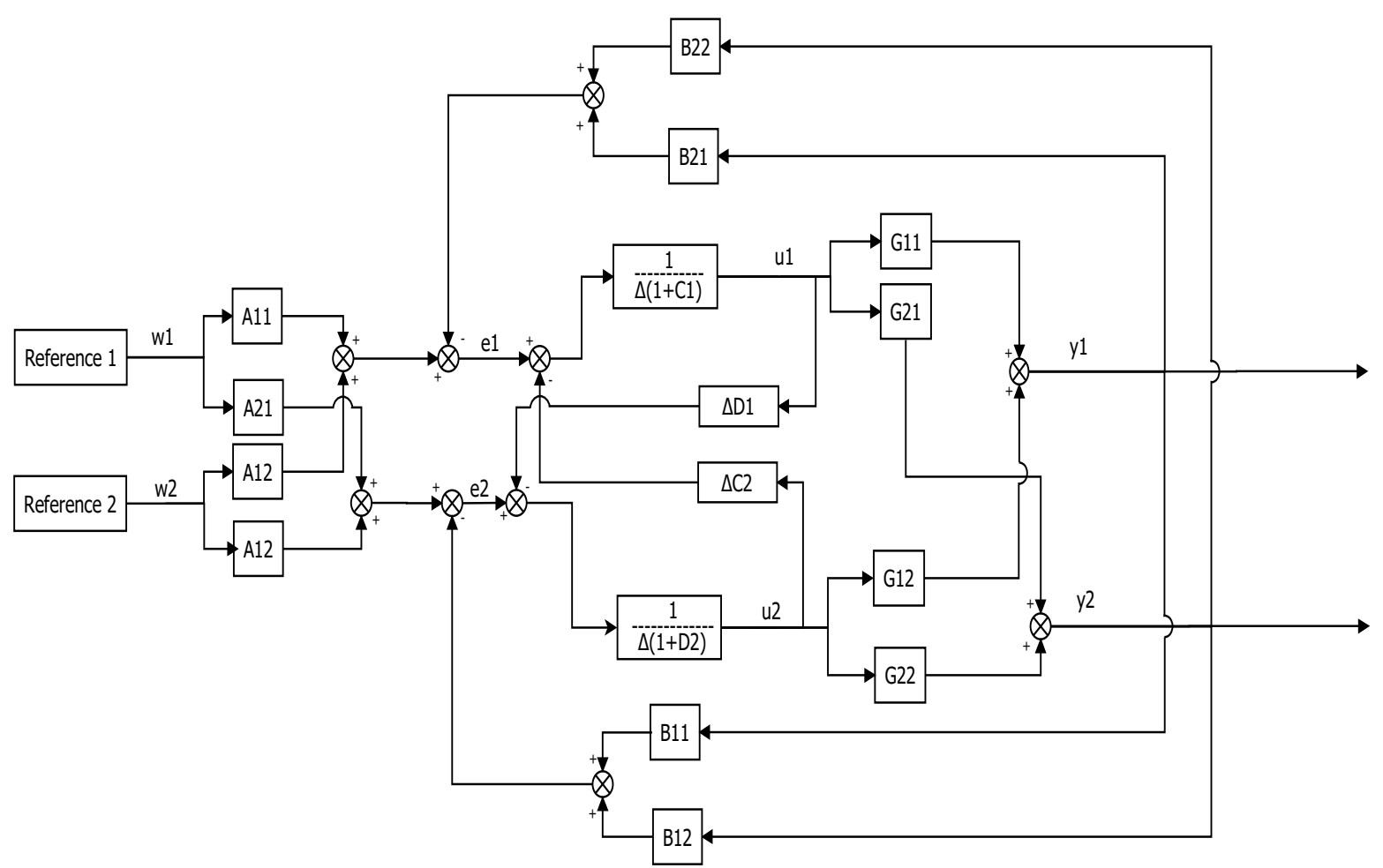

Figura 4.5: Representación LTI de un sistema controlado mediante DMC 


$$
\begin{aligned}
& \boldsymbol{\omega}=\left(\begin{array}{l}
\omega_{1} \\
\omega_{2}
\end{array}\right) \\
& \mathbf{y}=\left(\begin{array}{l}
\mathbf{y}_{1} \\
\mathbf{y}_{2}
\end{array}\right) \\
& \mathbf{u}=\left(\begin{array}{l}
\mathbf{u}_{1} \\
\mathbf{u}_{2}
\end{array}\right)
\end{aligned}
$$

De la Figura 4.5 se puede deducir que:

$$
\begin{gathered}
\boldsymbol{\omega} \mathbf{A}-\mathbf{B y}=\mathbf{e} \\
\mathbf{e}=\left(\begin{array}{l}
\mathrm{e}_{1} \\
\mathrm{e}_{2}
\end{array}\right)
\end{gathered}
$$

Reemplazado e en las Ecuaciones 4.28 y 4.29 :

$$
\begin{aligned}
& \mathrm{u}_{1}=\left(\mathrm{e}_{1}-\mathrm{C}_{1}\left(\mathrm{z}^{-1}\right) \Delta \mathrm{u}_{1}-\mathrm{C}_{2}\left(\mathrm{z}^{-1}\right) \Delta \mathrm{u}_{2}\right) \Delta^{-1} \\
& \mathrm{u}_{2}=\left(\mathrm{e}_{2}-\mathrm{D}_{1}\left(\mathrm{z}^{-1}\right) \Delta \mathrm{u}_{1}-\mathrm{D}_{2}\left(\mathrm{z}^{-1}\right) \Delta \mathrm{u}_{2}\right) \Delta^{-1}
\end{aligned}
$$

Y desarrollando las ecuaciones 4.31 y 4.32 un poco más, se halla la relación entre e and $\Delta \mathrm{u}$ :

$$
\begin{aligned}
& \Delta \mathrm{u}_{1}\left(1+\mathrm{C}_{1}\left(\mathrm{z}^{-1}\right)\right)+\mathrm{C}_{2}\left(\mathrm{z}^{-1}\right) \Delta \mathrm{u}_{2}=\mathrm{e}_{1} \\
& \Delta \mathrm{u}_{2}\left(1+\mathrm{D}_{2}\left(\mathrm{z}^{-1}\right)\right)+\mathrm{D}_{1}\left(\mathrm{z}^{-1}\right) \Delta \mathrm{u}_{1}=\mathrm{e}_{2}
\end{aligned}
$$

Expresando las Ecuaciones 4.33 y 4.34 en notación matricial:

$$
\begin{gathered}
\left(\begin{array}{cl}
\Delta\left(1+\mathrm{C}_{1}\left(\mathrm{z}^{-1}\right)\right) & \Delta \mathrm{C}_{2}\left(\mathrm{z}^{-1}\right) \\
\Delta \mathrm{D}_{1}\left(\mathrm{z}^{-1}\right) & \Delta\left(1+\mathrm{D}_{2}\left(\mathrm{z}^{-1}\right)\right)
\end{array}\right)\left(\begin{array}{l}
\mathrm{u}_{1} \\
\mathrm{u}_{2}
\end{array}\right)=\left(\begin{array}{l}
\mathrm{e}_{1} \\
\mathrm{e}_{2}
\end{array}\right) \\
\left(\begin{array}{l}
\mathrm{u}_{1} \\
\mathrm{u}_{2}
\end{array}\right)=\nabla\left(\begin{array}{l}
\mathrm{e}_{1} \\
\mathrm{e}_{2}
\end{array}\right)
\end{gathered}
$$

Donde $\nabla$ es:

$$
\begin{gathered}
\nabla=\left(\begin{array}{cl}
\Delta\left(1+\mathrm{C}_{1}\left(\mathrm{z}^{-1}\right)\right) & \Delta \mathrm{C}_{2}\left(\mathrm{z}^{-1}\right) \\
\Delta \mathrm{D}_{1}\left(\mathrm{z}^{-1}\right) & \Delta\left(1+\mathrm{D}_{2}\left(\mathrm{z}^{-1}\right)\right)
\end{array}\right)^{-1} \\
\nabla=\left(\begin{array}{ll}
\nabla_{11} & \nabla_{12} \\
\nabla_{21} & \nabla_{22}
\end{array}\right)
\end{gathered}
$$

En este punto, el diagrama de la Figura 4.5 se puede simplificar (Figura 4.6)

Usando notación matricial para todas las variables en la Ecuación 4.35 (Ecuación 4.39), la Figura 4.6 evoluciona a la Figura 4.4, alcanzando el objetivo de esta sección.

$$
(\boldsymbol{\omega} \mathbf{A}-\mathbf{y B}) \nabla=\mathbf{e} \nabla=\mathbf{u}
$$




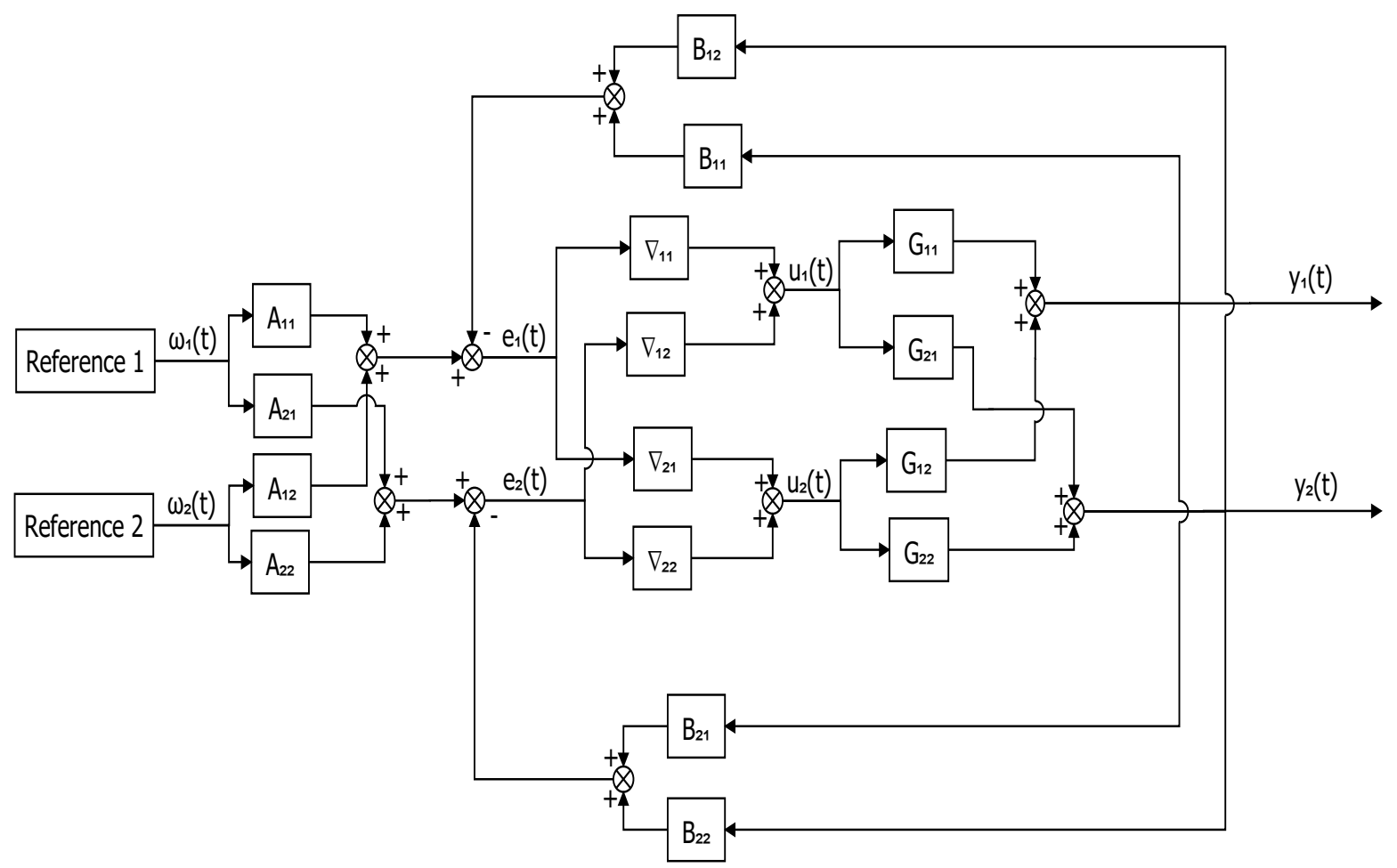

Figura 4.6: Representación LTI simplificada de un sistema controlado mediante DMC 
Solo falta calcular la matriz del sistema. Teniendo en cuenta que $\mathbf{G u}=\mathbf{y}$ y sustituyendo en la Ecuación 4.39:

$$
\mathbf{y}=(\mathbf{I}+\mathbf{G} \nabla \mathbf{B})^{-1} \mathbf{G} \nabla \mathbf{A} \boldsymbol{\omega}
$$

Luego la matriz del sistema es $\mathbf{H}=(\mathbf{I}+\mathbf{G} \nabla \mathbf{B})^{-1} \mathbf{G} \nabla \mathbf{A}$, y un sistema MIMO típico controlado mediante DMC puede expresarse como un lazo abierto (Figura 4.7 y Ecuación 4.41).

$$
\left(\begin{array}{l}
\mathrm{y}_{1} \\
\mathrm{y}_{2}
\end{array}\right)=\left(\begin{array}{ll}
\mathrm{H}_{11} & \mathrm{H}_{12} \\
\mathrm{H}_{21} & \mathrm{H}_{22}
\end{array}\right)\left(\begin{array}{l}
\omega_{1} \\
\omega_{2}
\end{array}\right)
$$

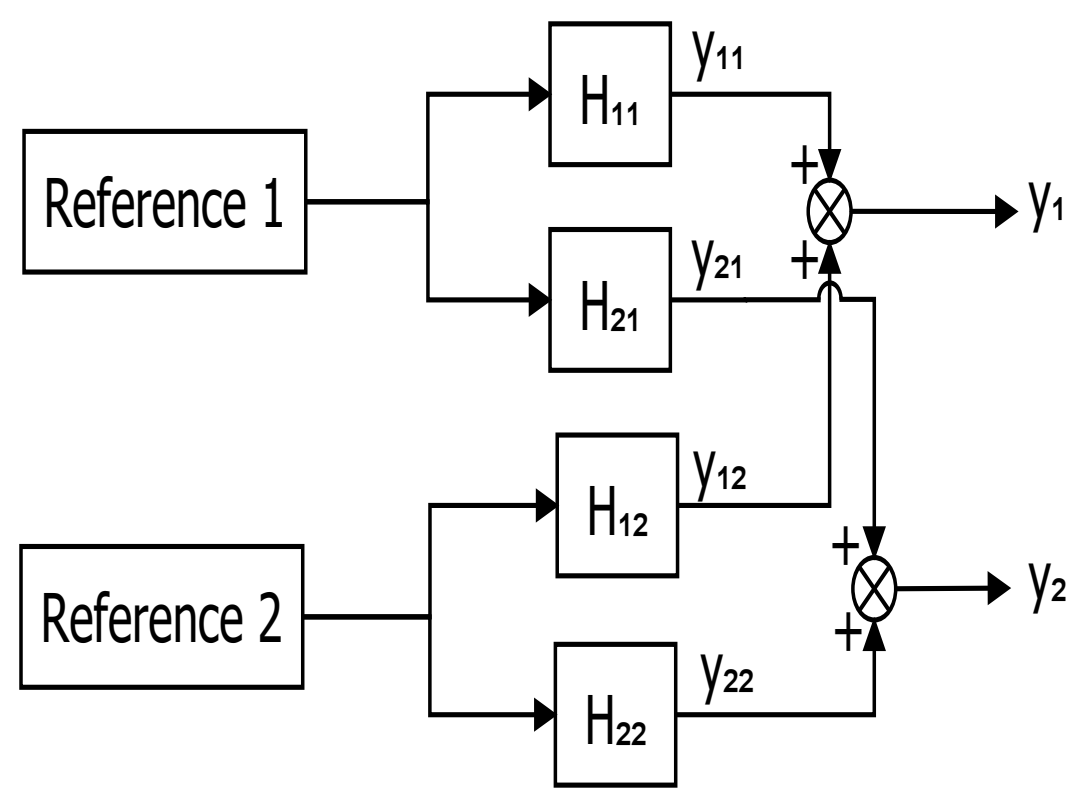

Figura 4.7: MIMO DMC en forma de sistema en lazo abierto

\subsection{Generalización a más variables}

En las secciones previas solo se ha desarrollado el caso MIMO 2x2 por simplicidad. Sin embargo, el procedimiento para sistemas con un mayor número de variables es el mismo. En esta sección se indicarán las líneas generales para expandir la formulación a sistemas mayores.

En el caso de un sistema [z x y], la Ecuación 4.10 debe modificarse para añadir el efecto de las nuevas variables: 


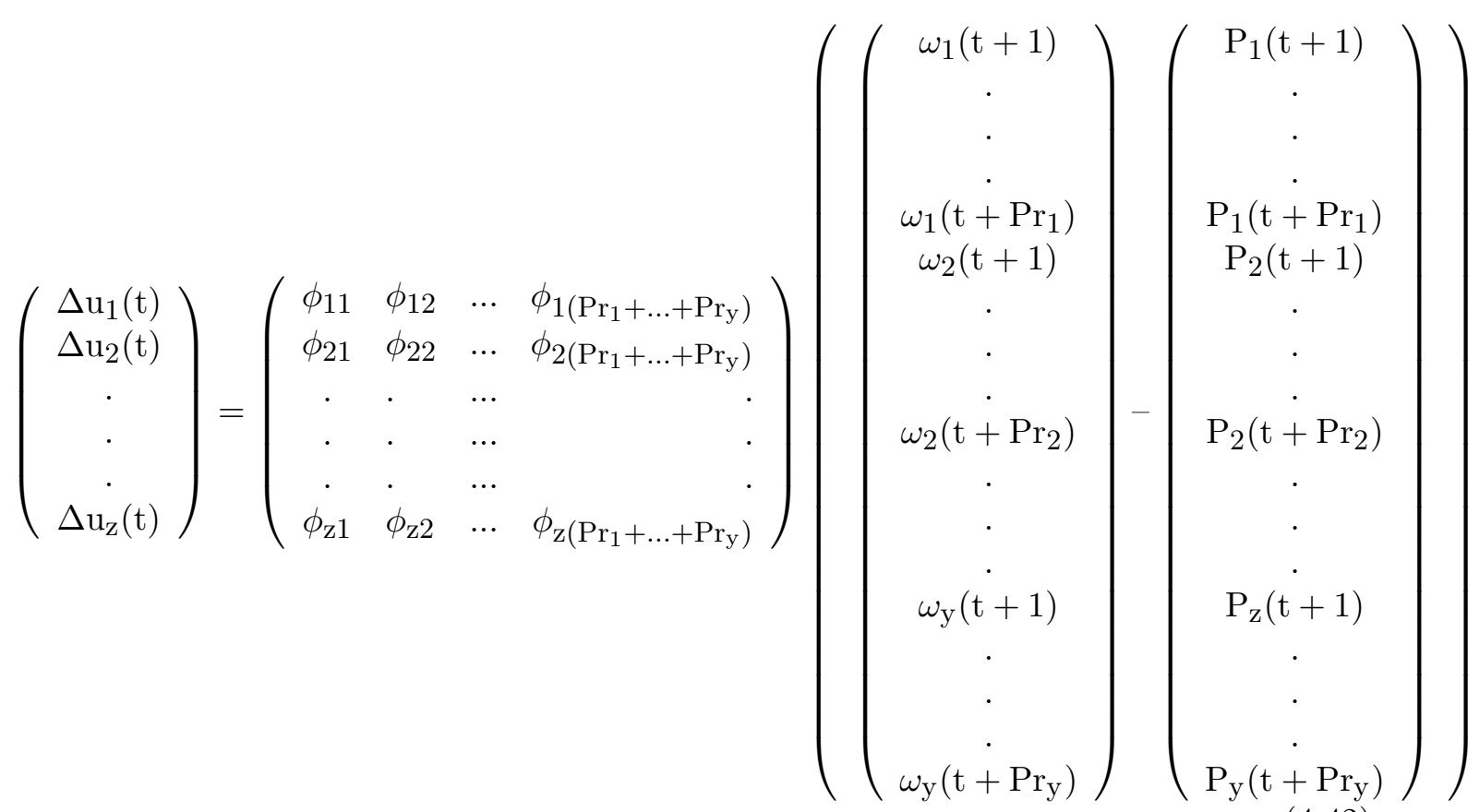

Luego el primer incremento de $\mathrm{u}_{1}$ (que es $\Delta \mathrm{u}_{1}(\mathrm{t})$ ) es:

$$
\begin{aligned}
& \Delta \mathrm{u}_{1}(\mathrm{t})=\left[\left(\sum_{\mathrm{i}=1}^{\operatorname{Pr}_{1}} \phi_{1 \mathrm{i}} \omega_{1}(\mathrm{t}+\mathrm{i})\right)+\left(\sum_{\mathrm{i}=\operatorname{Pr}_{1}+1}^{\operatorname{Pr}_{1}+\operatorname{Pr}_{2}} \phi_{1 \mathrm{i}} \omega_{2}\left(\mathrm{t}+\mathrm{i}-\operatorname{Pr}_{1}\right)\right)\right. \\
& \left.+\ldots+\left(\sum_{\mathrm{i}=\operatorname{Pr}_{1}+\ldots+\operatorname{Pr}_{\mathrm{y}-1}+1}^{\operatorname{Pr}_{1}+\ldots \operatorname{Pr}_{\mathrm{y}}} \phi_{1 \mathrm{i}} \omega_{\mathrm{y}}\left(\mathrm{t}+\mathrm{i}-\operatorname{Pr}_{\mathrm{y}}\right)\right)\right]-\left[\left(\sum_{\mathrm{i}=1}^{\operatorname{Pr}_{1}} \phi_{1 \mathrm{i}} \mathrm{P}_{1}(\mathrm{t}+\mathrm{i})\right)-\right. \\
& \left.\left(\sum_{\mathrm{i}=\operatorname{Pr}_{1}+1}^{\operatorname{Pr}_{1}+\operatorname{Pr}_{2}} \phi_{1 \mathrm{i}} \mathrm{P}_{2}\left(\mathrm{t}+\mathrm{i}-\operatorname{Pr}_{1}\right)\right)+\ldots+\left(\sum_{\mathrm{i}=\operatorname{Pr}_{1}+\ldots+\operatorname{Pr}_{\mathrm{y}-1}+1}^{\operatorname{Pr}_{1}+\ldots \operatorname{Pr}_{\mathrm{y}}} \phi_{1 \mathrm{i}} \mathrm{P}_{\mathrm{y}}\left(\mathrm{t}+\mathrm{i}-\operatorname{Pr}_{\mathrm{y}}\right)\right)\right]
\end{aligned}
$$

Añadiendo la formulación completa de la respuesta libre a la Ecuación 4.43, se obtiene la Ecuación 4.44. 


$$
\begin{aligned}
\Delta \mathrm{u}_{1}(\mathrm{t})= & {\left[\left(\sum_{\mathrm{i}=1}^{\operatorname{Pr}_{1}} \phi_{1 \mathrm{i}} \omega_{1}(\mathrm{t}+\mathrm{i})\right)+\left(\sum_{\mathrm{i}=\operatorname{Pr}_{1}+1}^{\operatorname{Pr}_{1}+\operatorname{Pr}_{2}} \phi_{1 \mathrm{i}} \omega_{2}\left(\mathrm{t}+\mathrm{i}-\operatorname{Pr}_{1}\right)\right)+\ldots+\left(\sum_{\mathrm{i}=\operatorname{Pr}_{1}+\ldots+\operatorname{Pr}_{\mathrm{y}-1}+1}^{\operatorname{Pr}_{1}+\ldots \operatorname{Pr}_{\mathrm{y}}} \phi_{1 \mathrm{i}} \omega_{\mathrm{z}}\left(\mathrm{t}+\mathrm{i}-\operatorname{Pr}_{\mathrm{y}}\right)\right)\right]-} \\
& {\left[\left(\sum_{\mathrm{i}=1}^{\operatorname{Pr}_{1}} \phi_{1 \mathrm{i}}\left(\mathrm{y}_{1, \mathrm{~m}}(\mathrm{t})+\sum_{\mathrm{j}=1}^{\mathrm{z}}\left(\mathrm{S}_{\mathrm{n}, 1 \mathrm{j}}^{\mathrm{i}}\left(\mathrm{z}^{-1}\right) \Delta \mathrm{u}_{\mathrm{j}}\right)\right)\right)+\left(\sum_{\mathrm{i}=\operatorname{Pr}_{1}+1}^{\operatorname{Pr}_{1}+\operatorname{Pr}_{2}} \phi_{1 \mathrm{i}}\left(\mathrm{y}_{2, \mathrm{~m}}(\mathrm{t})+\sum_{\mathrm{j}=1}^{\mathrm{z}}\left(\mathrm{S}_{\mathrm{n}, 2 \mathrm{j}}^{\mathrm{i}}\left(\mathrm{z}^{-1}\right) \Delta \mathrm{u}_{\mathrm{j}}\right)\right)\right)\right.} \\
& \left.+\ldots+\left(\sum_{\mathrm{i}=\operatorname{Pr}_{1}+\ldots+\operatorname{Pr}_{\mathrm{y}-1}+1}^{\operatorname{Pr}_{1}+\ldots \operatorname{Pr}_{\mathrm{y}}} \phi_{\mathrm{zi}}\left(\mathrm{y}_{\mathrm{y}, \mathrm{m}}(\mathrm{t})+\sum_{\mathrm{j}=1}^{\mathrm{z}}\left(\mathrm{S}_{\mathrm{n}, \mathrm{zj}}^{\mathrm{i}}\left(\mathrm{z}^{-1}\right) \Delta \mathrm{u}_{\mathrm{j}}\right)\right)\right)\right]
\end{aligned}
$$

Y haciendo simplificaciones similares a las realizadas en la sección 4.2 (ver Apéndice A):

$$
\begin{aligned}
\Delta \mathrm{u}_{1}(\mathrm{t})= & \mathrm{A}_{11} \omega_{1}(\mathrm{t})+\mathrm{A}_{12} \omega_{2}(\mathrm{t})-\mathrm{B}_{11} \mathrm{y}_{1}(\mathrm{t})-\mathrm{B}_{12} \mathrm{y}_{2}(\mathrm{t})-\mathrm{C}_{1}\left(\mathrm{q}^{-1}\right) \Delta \mathrm{u}_{1}(\mathrm{t})-\mathrm{C}_{2}\left(\mathrm{z}^{-1}\right) \Delta \mathrm{u}_{2}(\mathrm{t}) \\
& +\ldots+\mathrm{A}_{1 \mathrm{z}} \omega_{\mathrm{z}}(\mathrm{t})-\mathrm{B}_{1 \mathrm{z}} \mathrm{y}_{\mathrm{z}}(\mathrm{t})-\mathrm{C}_{\mathrm{z}}\left(\mathrm{z}^{-1}\right) \Delta \mathrm{u}_{\mathrm{z}}(\mathrm{t}) \\
\Delta \mathrm{u}_{2}(\mathrm{t})= & \mathrm{A}_{21} \omega_{1}(\mathrm{t})+\mathrm{A}_{22} \omega_{2}(\mathrm{t})-\mathrm{B}_{21} \mathrm{y}_{1}(\mathrm{t})-\mathrm{B}_{22} \mathrm{y}_{2}(\mathrm{t})-\mathrm{D}_{1}\left(\mathrm{z}^{-1}\right) \Delta \mathrm{u}_{1}(\mathrm{t})-\mathrm{D}_{2}\left(\mathrm{z}^{-1}\right) \Delta \mathrm{u}_{2}(\mathrm{t}) \\
& +\ldots+\mathrm{A}_{2 \mathrm{z}} \omega_{\mathrm{z}}(\mathrm{t})-\mathrm{B}_{2 \mathrm{z}} \mathrm{y}_{\mathrm{z}}(\mathrm{t})-\mathrm{D}_{\mathrm{z}}\left(\mathrm{z}^{-1}\right) \Delta \mathrm{u}_{\mathrm{z}}(\mathrm{t})
\end{aligned}
$$

$\Delta \mathrm{u}_{\mathrm{z}}(\mathrm{t})=\mathrm{A}_{\mathrm{z} 1} \omega_{1}(\mathrm{t})-\mathrm{B}_{\mathrm{z} 1} \mathrm{y}_{1}(\mathrm{t})-\mathrm{Z}_{1}\left(\mathrm{z}^{-1}\right) \Delta \mathrm{u}_{1}(\mathrm{t})+\ldots+\mathrm{A}_{\mathrm{zz}} \omega_{\mathrm{z}}(\mathrm{t})-\mathrm{B}_{\mathrm{z} 1} \mathrm{y}_{1}(\mathrm{t})-\mathrm{Z}_{\mathrm{z}}\left(\mathrm{z}^{-1}\right) \Delta \mathrm{u}_{\mathrm{z}}(\mathrm{t})$

Añadir nuevas variables implica añadir nuevos polinomios en $\mathrm{z}^{-1}$. Esto hace más complejo el cálculo de la solución final. Sin embargo, el método en si mismo es sencillo y puede usarse con cualquier número de variables sin cambios.

\subsection{Validación}

Para demostrar la exactitud de la formulación desarrollada y comprobar el efecto de los errores numéricos en los cálculos desarrollados en las secciones anteriores, se va a proceder a comparar los resultados de tres benchmarks controlados por DMC usando la formulación LTI y el algoritmo DMC. Los benchmarks seleccionados son:

1. Benchmark 1 (Columna de destilación de Wood Berry, [54]).

$$
\mathbf{G}=\left(\begin{array}{cc}
\frac{12.8 \mathrm{e}^{-\mathrm{s}}}{(16.7 \mathrm{~s}+1)} & \frac{-18.9 \mathrm{e}^{-3 \mathrm{~s}}}{(21 \mathrm{~s}+1)} \\
\frac{6.6 \mathrm{e}^{-7 \mathrm{~s}}}{(10.9 \mathrm{~s}+1)} & \frac{-19.4 \mathrm{e}^{-3 \mathrm{~s}}}{(14.4 \mathrm{~s}+1)}
\end{array}\right)
$$


2. Benchmark 2 (Motor-alternador, [55]).

$$
\mathbf{G}=\left(\begin{array}{cl}
\frac{6.1}{(\mathrm{~s}+7)} & \frac{4.6}{(\mathrm{~s}+14.3)} \\
\frac{2.8}{(\mathrm{~s}+6.8)} & \frac{-0.24}{(\mathrm{~s}+4)}
\end{array}\right)
$$

3. Benchmark 3 (Columna de destilación binaria, [56]).

$$
\mathbf{G}=\left(\begin{array}{cc}
\frac{0.878}{(75 \mathrm{~s}+1)} & \frac{-0.864}{(75 \mathrm{~s}+1)} \\
\frac{1.082}{(75 \mathrm{~s}+1)} & \frac{-1.096}{(75 \mathrm{~s}+1)}
\end{array}\right)
$$

Se usarán dos conjuntos de parámetros para cada benchmark (Cuadros 4.2,4.3,4.4). Por simplicidad, se emplearán los mismos horizontes de predicción modelo y control para cada variable.

\begin{tabular}{|c|c|c|c|c|}
\hline Conjunto & Horizonte de predicción & Horizonte de control & Horizonte de modelo & Factor de peso \\
\hline 1 & 20 & 1 & 140 & 1 \\
\hline 2 & 7 & 1 & 140 & 0.2 \\
\hline
\end{tabular}

Cuadro 4.2: Conjunto de parámetros para Benchmark 1

\begin{tabular}{|c|c|c|c|c|}
\hline Conjunto & Horizonte de predicción & Horizonte de control & Horizonte de modelo & Factor de peso \\
\hline 1 & 5 & 2 & 120 & 0.002 \\
\hline 2 & 10 & 2 & 120 & 0.2 \\
\hline
\end{tabular}

Cuadro 4.3: Conjunto de parámetros para Benchmark 2

\begin{tabular}{|c|c|c|c|c|}
\hline Conjunto & Horizonte de predicción & Horizonte de control & Horizonte de modelo & Factor de peso \\
\hline 1 & 60 & 2 & 150 & 0.05 \\
\hline 2 & 40 & 2 & 150 & 0.04 \\
\hline
\end{tabular}

Cuadro 4.4: Conjunto de parámetros para Benchmark 3

Las Figuras 4.8, 4.9 y 4.10 representan las respuestas temporales para el algoritmo DMC y el equivalente LTI propuesto. Para todos los benchmarks y conjuntos de parámetros, la respuesta temporal es exactamente la misma para ambas formulaciones, demostrando que se ha cumplido el objetivo de esta sección (encontrar una formulación LTI completamente equivalente al algoritmo DMC).

\subsection{DMC como desacoplador}

Como ejemplo de aplicación de la formulación propuesta, se va a realizar un estudio del efecto desacoplador del DMC en esta sección. 

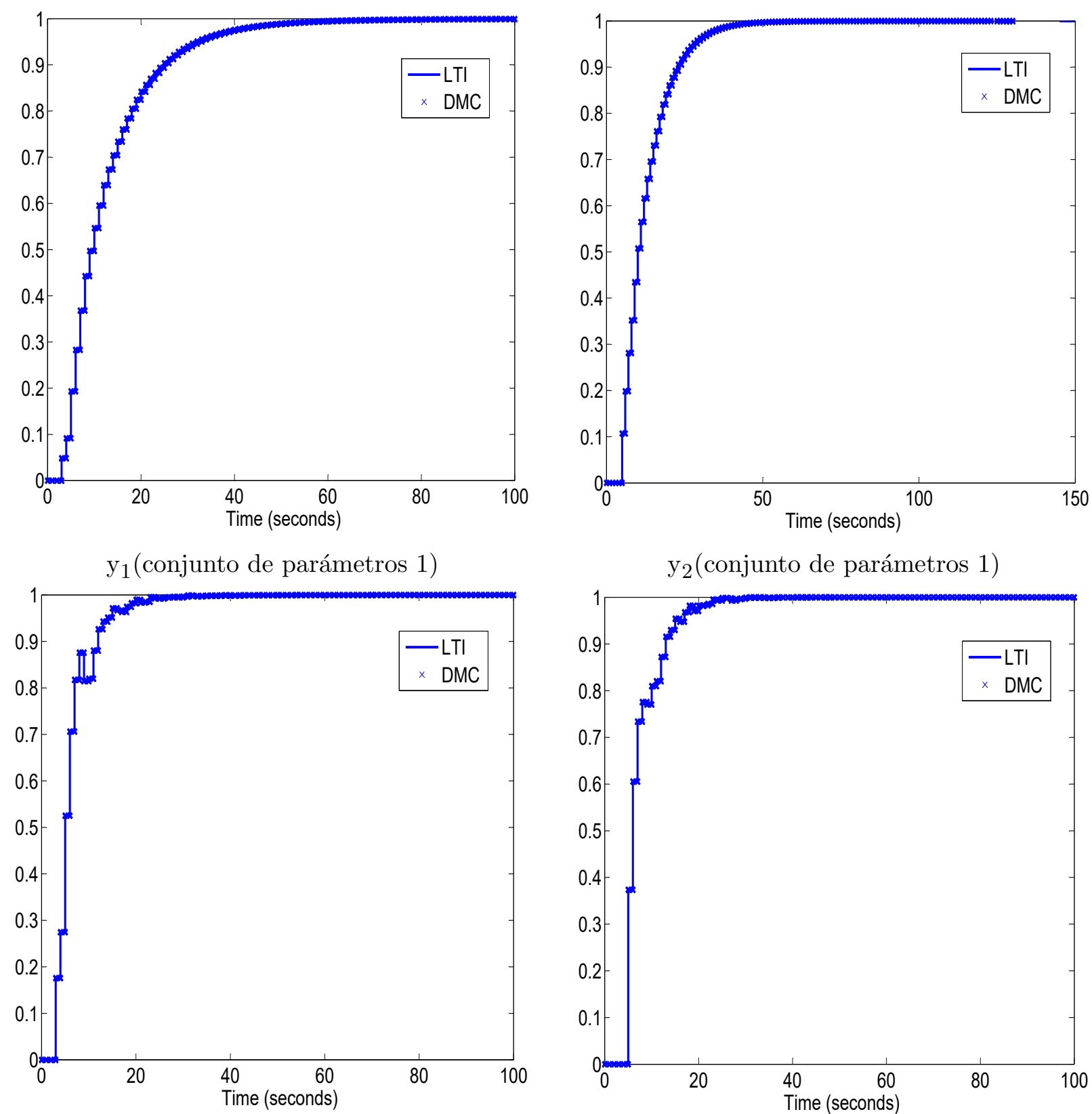

$\mathrm{y}_{1}$ (conjunto de parámetros 2)

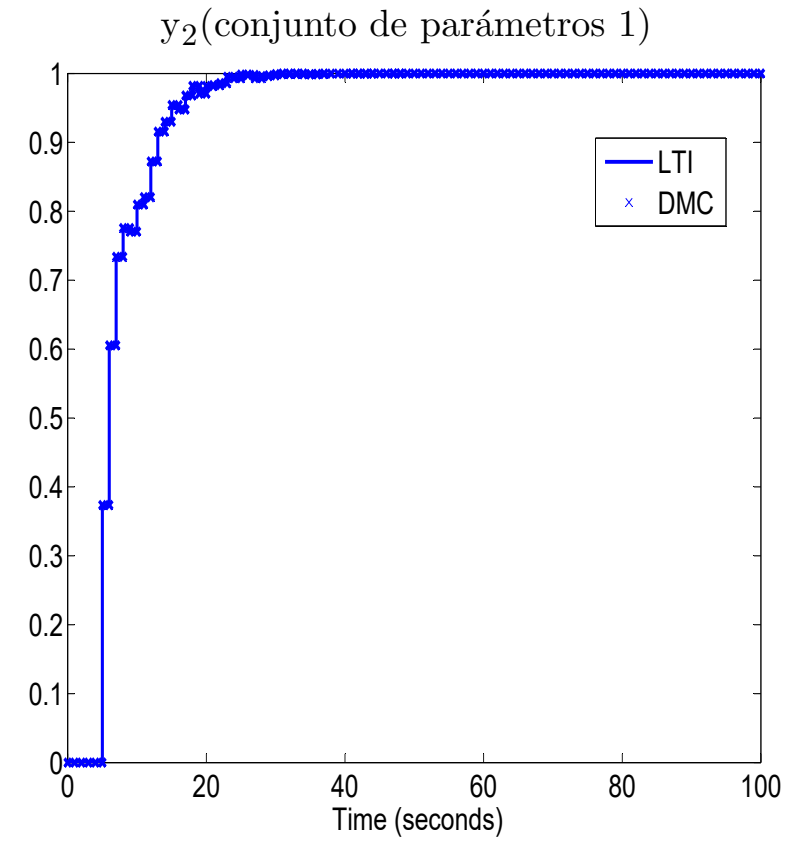

$\mathrm{y}_{2}$ (conjunto de parámetros 2)

Figura 4.8: Comparación de métodos para el benchmark 1 

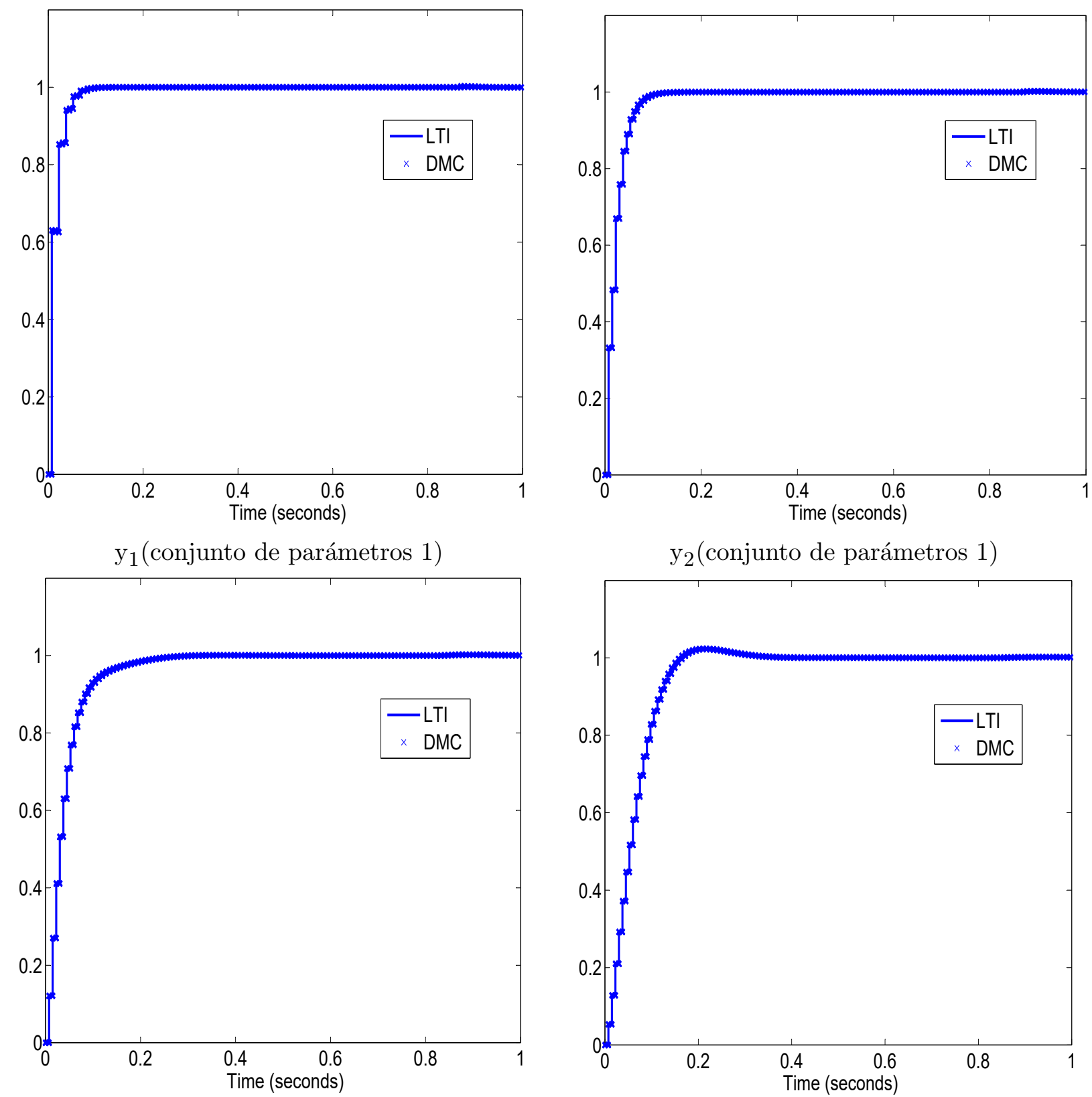

$\mathrm{y}_{1}$ (conjunto de parámetros 2)

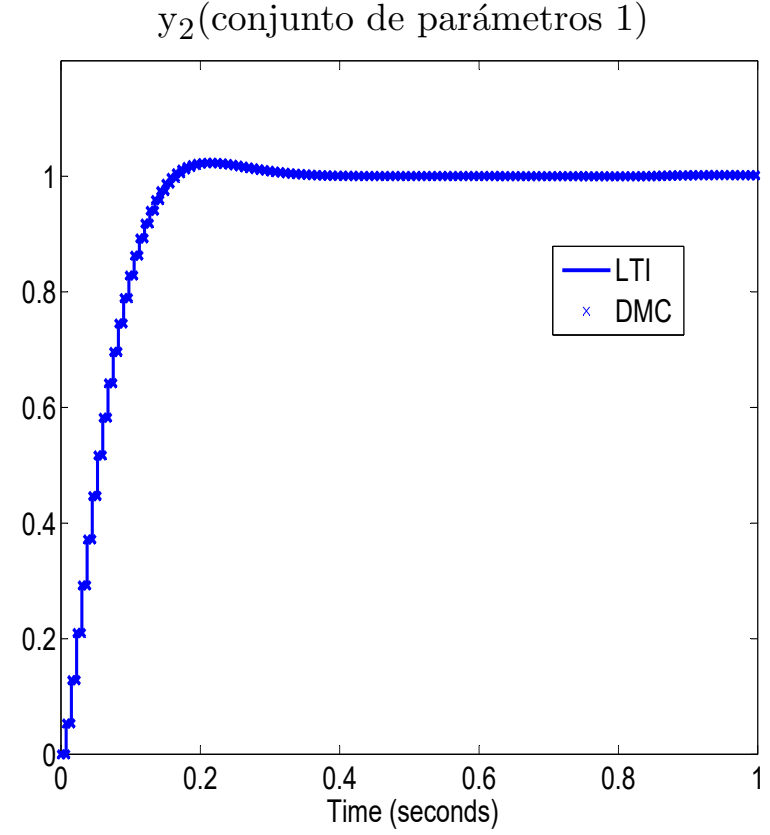

$\mathrm{y}_{2}($ conjunto de parámetros 2$)$

Figura 4.9: Comparación de métodos para el benchmark 2 

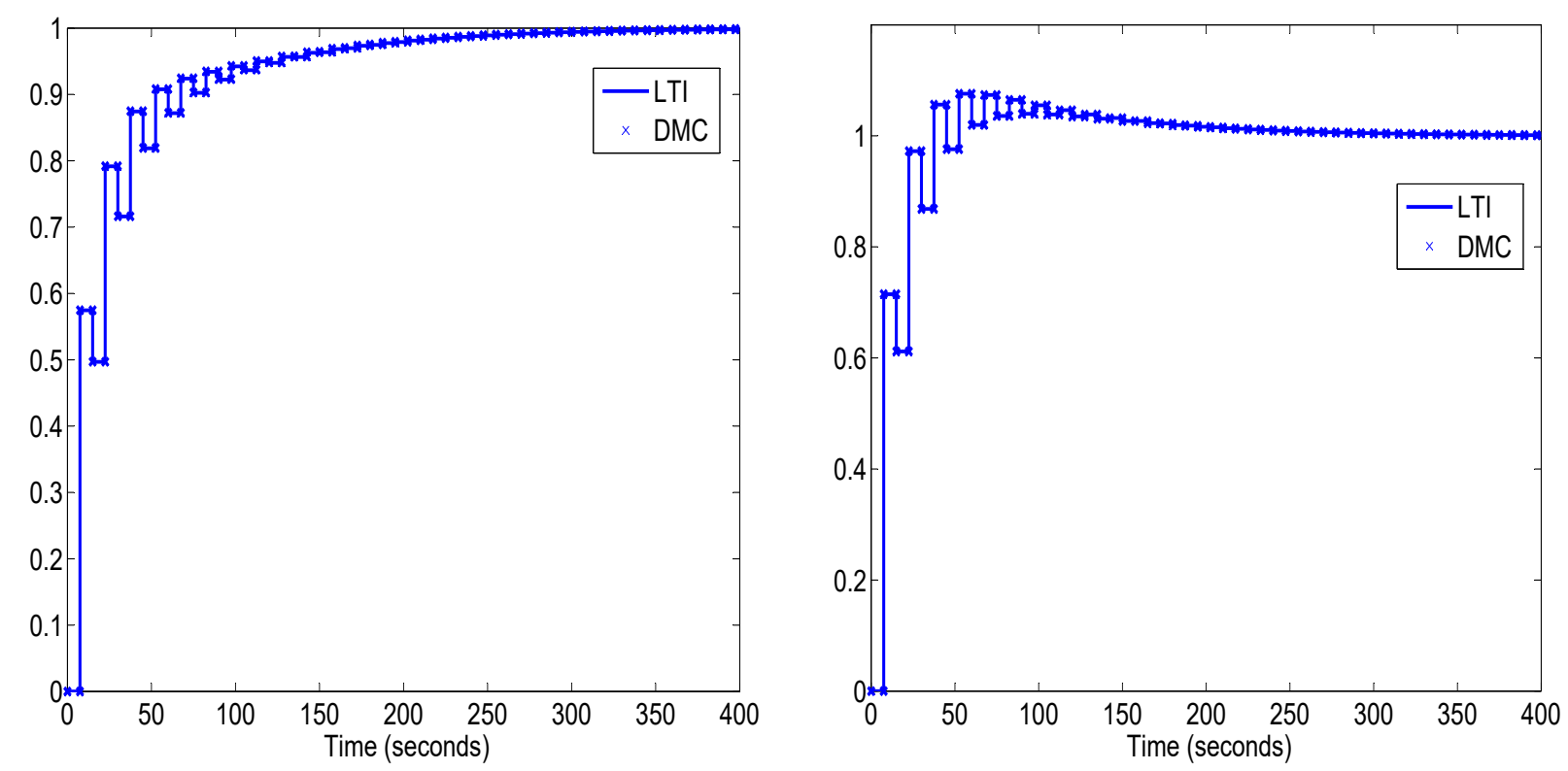

$\mathrm{y}_{1}$ (conjunto de parámetros 1)

$\mathrm{y}_{2}$ (conjunto de parámetros 1$)$

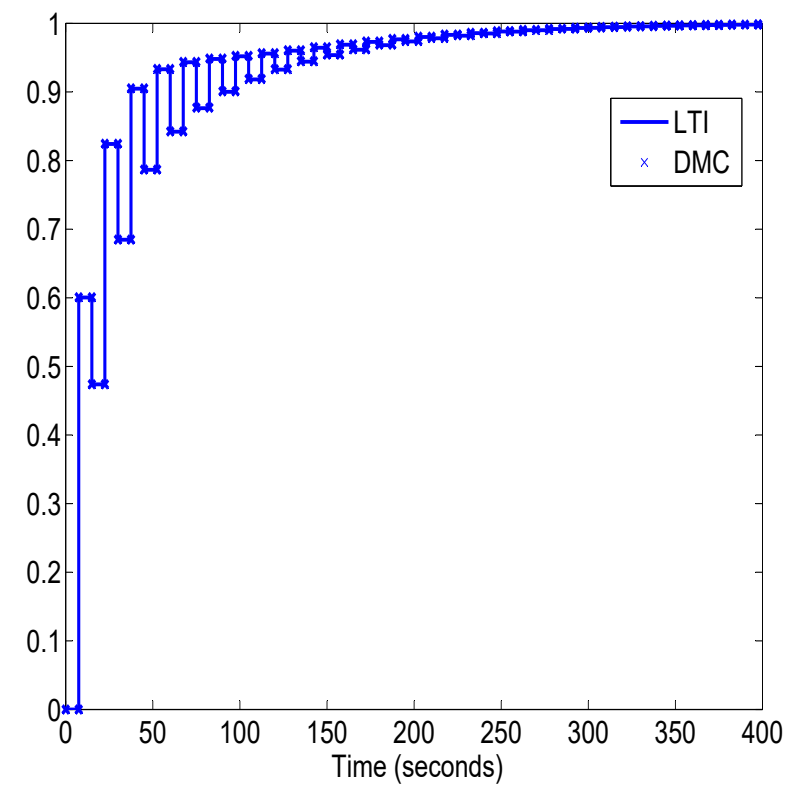

$\mathrm{y}_{1}$ (conjunto de parámetros 2$)$

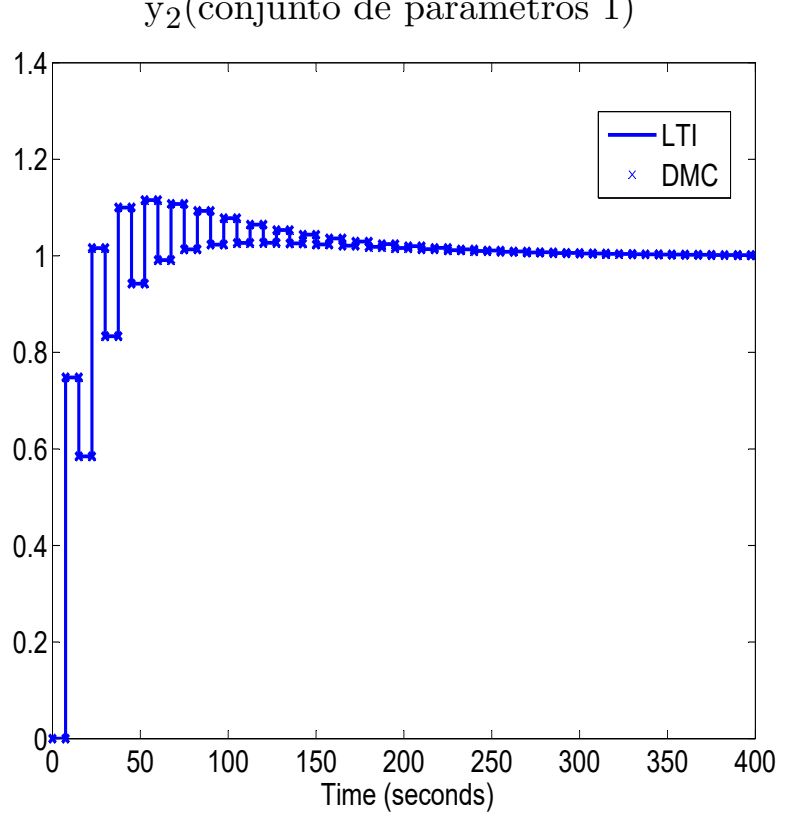

$\mathrm{y}_{2}$ (conjunto de parámetros 2)

Figura 4.10: Comparación de métodos para el benchmark 3 
En sistemas MIMO es interesante desacoplar las entradas y las salidas para conseguir que cada salida dependa de una entrada. Para medir la posibilidad de conseguir esto en un sistema, se calcula la matriz de ganancias relativas (Relative Gain Array, RGA). Este método fue introducido en [57] y se explica con detalle, por ejemplo, en [58]. Aquí se dará un breve resumen.

Sea H(s) la matriz de transferencia de un sistema MIMO. La matriz RGA se calcula según la Ecuación 4.51.

$$
\Lambda=\mathrm{H}(\mathrm{s}) \times\left(\mathrm{H}(\mathrm{s})^{\mathrm{T}}\right)^{-1}
$$

Los elementos de $\Lambda\left(\lambda_{\mathrm{ij}}\right)$ indican el grado de las interacciones para cada posible lazo de control dentro de un sistema MIMO. A partir de esta información será posible saber si hay lazos desacoplados del resto o no.

- $\lambda_{\mathrm{ij}}=1$ la ganancia en lazo abierto es igual a la ganancia en lazo cerrado. La entrada i afecta a la salida j sin interacciones de otras entradas. Están perfectamente desacopladas.

- $\lambda_{\mathrm{ij}}=0$. La ganancia en lazo cerrado es cero. La entrada i no tiene efecto en la salida $\mathrm{j}$.

- $0<\lambda_{\mathrm{ij}}<1$. Las interacciones en lazo cerrado aumentan la ganancia. Este caso puede dividirse:

- $\lambda_{\mathrm{ij}}<0,5$. El efecto de las interacciones es superior al de la vía principal.

- $\lambda_{\mathrm{ij}}=0,5$. Las interacciones y la vía principal tienen el mismo efecto.

- $\lambda_{\mathrm{ij}}>0,5$. El efecto de la vía principal es superior al de las interacciones.

- $\lambda_{\mathrm{ij}}>1$. Las interacciones hacen que la ganancia disminuya. Cuanto mayor sea el valor de $\lambda_{\mathrm{ij}}$, más importantes serán.

- $\lambda_{\mathrm{ij}}<0$. La ganancia en lazo cerrado funciona en sentido opuesto a la ganancia en lazo abierto. Es una situación potencialmente inestable y que debe evitarse un lazo de control independiente entre la entrada i y salida j.

Puede deducirse que un sistema con una matriz RGA igual a la matriz identidad es más fácil de controlar porque entradas y salidas están perfectamente desacopladas y no existen interacciones. No es complicado entender que el DMC actúa como un desacoplador, pero la demostración matemática de esto es elusiva, ya que no se puede calcular la matriz RGA de un algoritmo. Pero si se usa la formulación descrita en este capítulo, es fácil obtener esta matriz y demostrar esta afirmación. Si se calcula la matriz del sistema descrita en la Ecuación 4.41 para los tres benchmarks usados como ejemplo en la sección anterior (para cualquier conjunto de parámetros) y después se calcula la matriz RGA, se obtiene siempre el mismo resultado: 


$$
\boldsymbol{\Lambda}=\left(\begin{array}{ll}
1 & 0 \\
0 & 1
\end{array}\right)
$$

Las Figuras 4.11, 4.12 y 4.13 muestran las salidas las diferente funciones de transferencia (Ecuaciones 4.41 y Figura 4.7) de los benchmarks 1, 2 y 3 . Puede verse que las interacciones entre las entrada 1 y la salida 2 y entre la entrada 2 y la salida 1 solo aparecen en la respuesta transitoria y tienden a cero en estado estacionario.

Las Figuras 4.11, 4.12 y 4.13 y el hecho de que la matriz RGA siempre sea igual a la matriz identidad demuestran que el DMC actúa como un desacoplador en sistemas MIMO.
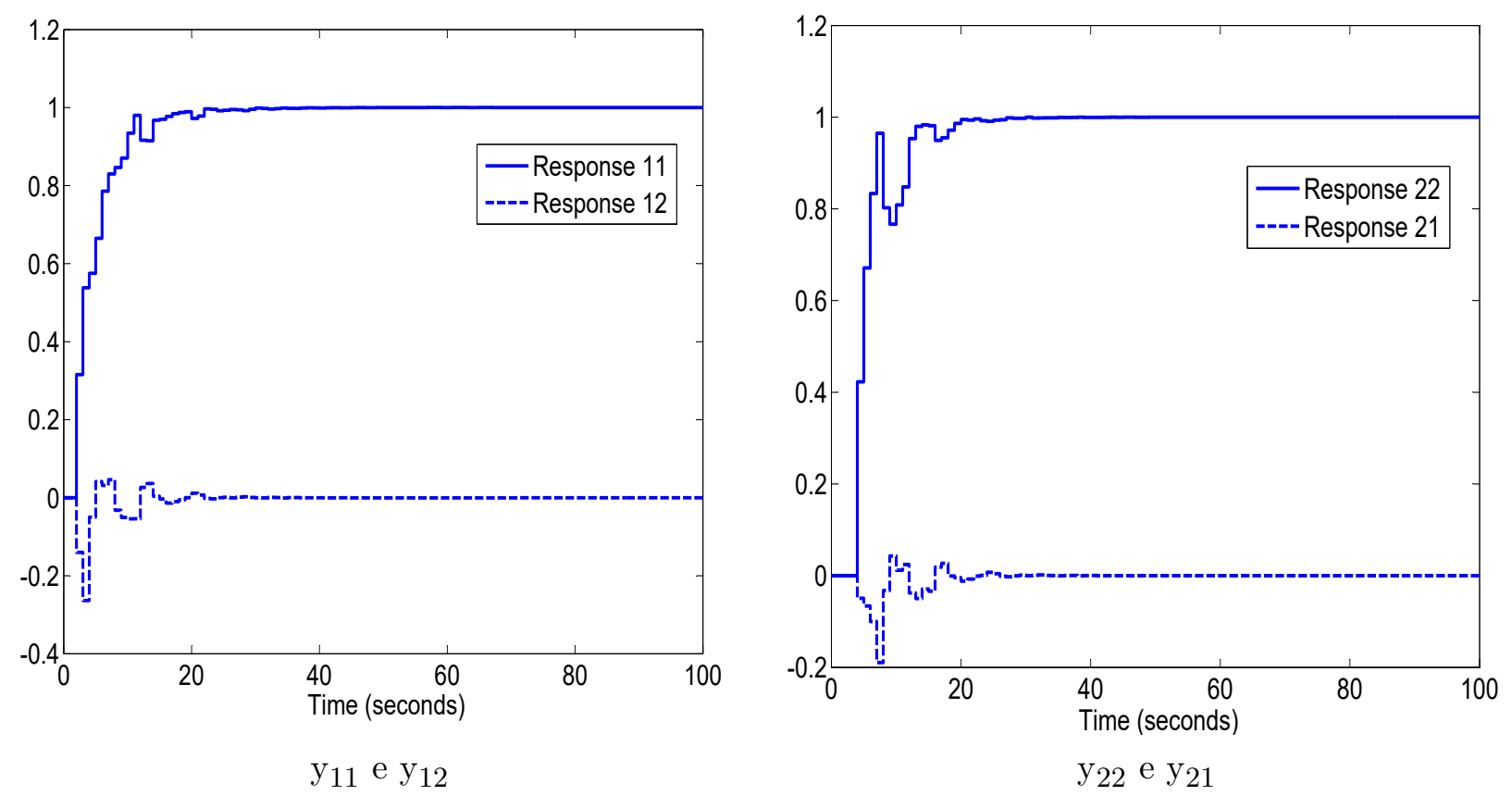

Figura 4.11: Benchmark 1 Salidas para el conjunto de parámetros 2

\subsection{Conclusiones}

En este capítulo se ha presentado una formulación LTI para DMC multivariable sin restricciones. Se ha usado la solución básica del DMC para un caso [2x2] como punto de partida del desarrollo de un conjunto de expresiones que pueden traducirse a un diagrama de bloques LTI. Simplificando este diagrama permite obtener una formulación LTI y un diagrama de bloques definitivo. La formulación LTI da las funciones de transferencia en lazo cerrado para las relaciones directas $\left(\mathrm{H}_{\mathrm{xx}}\right)$ y los acoplamientos $\left(\mathrm{H}_{\mathrm{xy}}\right)$. Esta formulación se ha expandido a sistemas de orden superior a [2x2].

Una vez se ha calculado la formulación LTI, se ha puesto a prueba. Se ha obtenido la respuesta temporal de tres benchmarks con el algoritmo DMC y la formulación LTI propuesta para dos conjuntos de parámetros. En todos los casos, la respuesta temporal para 


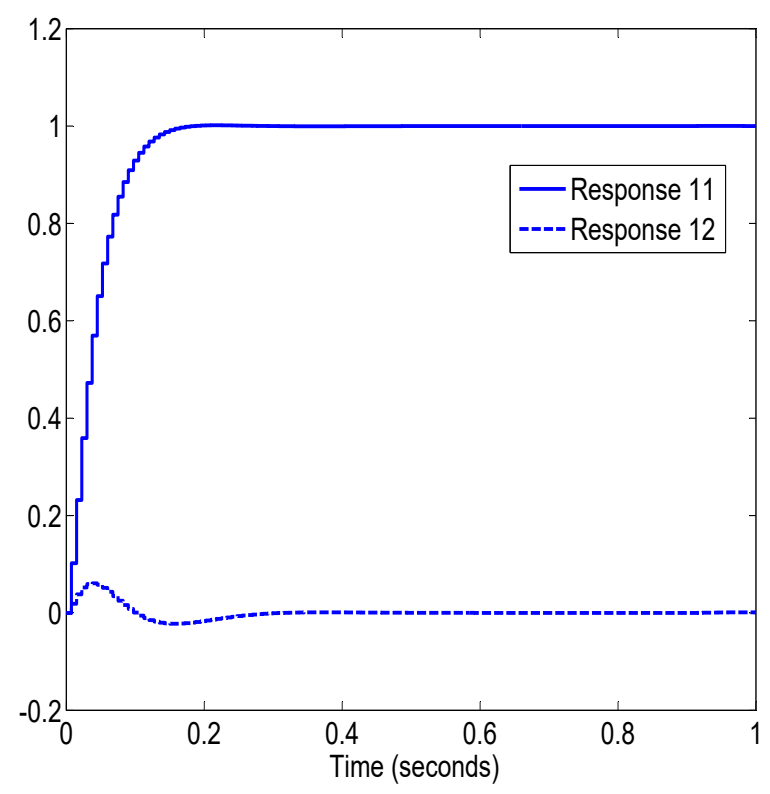

$\mathrm{y}_{11}$ e $\mathrm{y}_{12}$

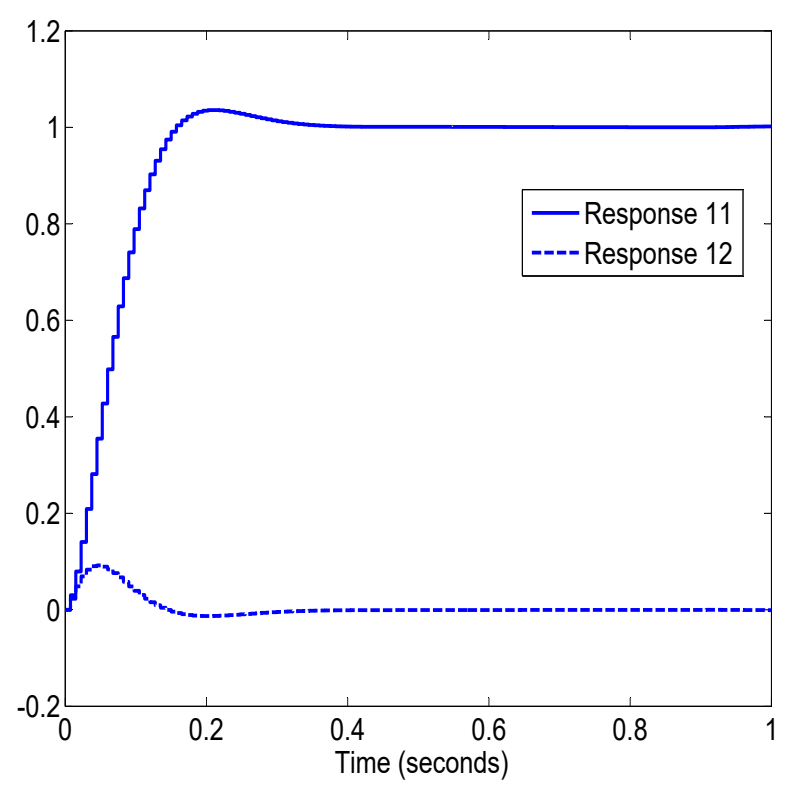

$\mathrm{y}_{22}$ e $\mathrm{y}_{21}$

Figura 4.12: Benchmark 2 Salidas para el conjunto de parámetros 2

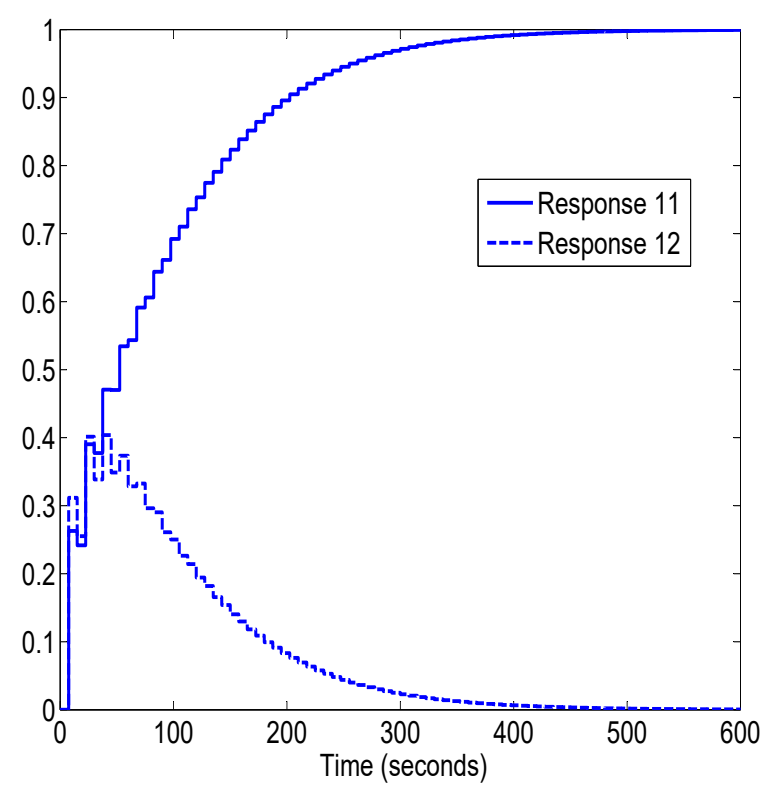

$\mathrm{y}_{11}$ e $\mathrm{y}_{12}$

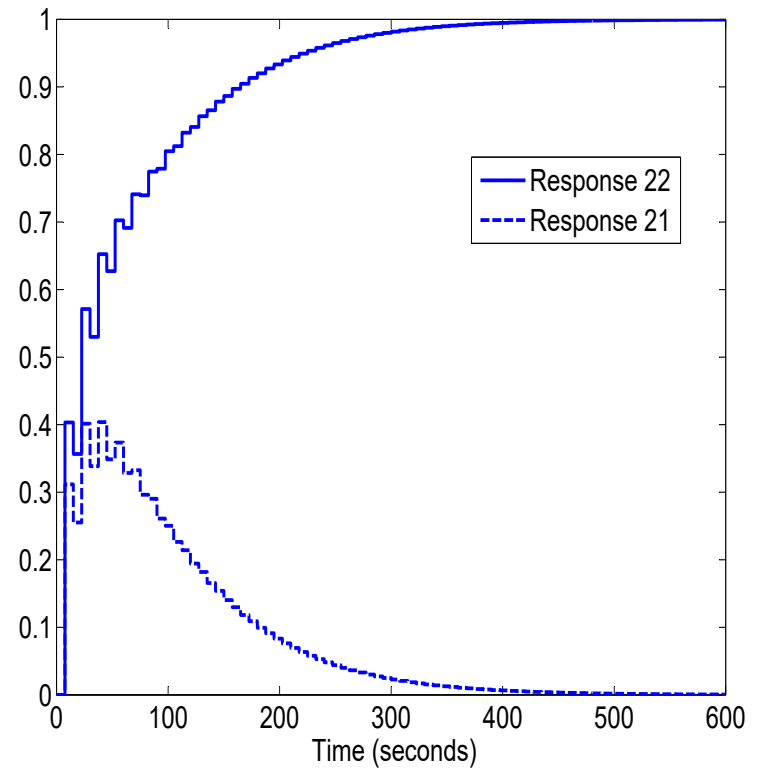

$\mathrm{y}_{22}$ e $\mathrm{y}_{21}$

Figura 4.13: Benchmark 3 Salidas para el conjunto de parámetros 1 
ambos métodos es idéntica, demostrando que la formulación propuesta es equivalente al algoritmo original.

La formulación LTI ha permitido demostrar que el DMC actúa como un desacoplador en la sección 4.5 . 


\section{Capítulo 5}

\section{Reglas de diseño para sistemas MIMO}

\subsection{Introducción}

En el capítulo 2 se desarrollaron una serie de reglas de diseño para DMC monovariable, a partir del estudio del movimiento de los polos facilitado por la formulación LTI. Una vez que se ha obtenido una formulación LTI para sistemas multivariable, estamos en posición de hacer el mismo estudio y desarrollar reglas de diseño para sistemas multivariable controlados mediante DMC.

Tal y como se ha explicado en el capítulo 4, la mayoría de procesos industriales son multivariable. Sin embargo, la búsqueda de reglas de diseño para controladores predictivos se ha centrado en el caso monovariable, existiendo apenas casos de reglas de diseño para el caso multivariable ([17], [7], [48]). El objetivo de este capítulo es obtener una serie de reglas de diseño para sistemas multivariables controlados mediante DMC que proporcionen unas prestaciones, no necesariamente óptimas, pero que puedan ser utilizadas como ajuste un inicial.

Para alcanzar este objetivo, se centrará el estudio en uno de los benchmarks utilizados como ejemplo en el capítulo anterior, el motor alternador. Se estudiará la influencia de los parámetros en los polos en lazo cerrado para comprobar si las reglas dadas en el capítulo 3 son aplicables a casos multivariable, y, si no lo son, modificarlas en consecuencia.

$$
\mathbf{G}=\left(\begin{array}{ll}
\frac{6.1}{(\mathrm{~s}+7)} & \frac{4.6}{(\mathrm{~s}+14.3)} \\
\frac{2.8}{(\mathrm{~s}+6.8)} & \frac{-0.24}{(\mathrm{~s}+4)}
\end{array}\right)
$$

\subsection{Influencia del horizonte de predicción}

En el capítulo 3 se pudo comprobar que el efecto del horizonte de predicción en los polos del sistema consistía en reforzar la predominancia de los polos reales y disminuir la de los polos complejos. En la respuesta temporal esto se traduce en una respuesta libre de oscilaciones pero cada vez más lenta a medida que aumenta el horizonte de predicción. 
Utilizando uno de los benchmark usados en el capítulo anterior (Motor-alternador, [55]) se va a estudiar el efecto del horizonte de predicción en los polos y la respuesta temporal. En primer lugar se variará $\operatorname{Pr}_{1}$ de 2 a 10 dejando $\operatorname{Pr}_{2}$ en un valor bajo para minimizar su efecto.El horizonte de predicción y el factor de peso también se mantendrán fijos $(\mathrm{M}=2$ y $\lambda=0,2)$. Las Figuras 5.1 y 5.2 muestran la evolución de los polos en lazo cerrado de cada una de las funciones de transferencia cuando se varía uno de los horizontes de predicción. Como puede verse, al aumentar el horizonte de predicción los polos complejos empiezan a alejarse del círculo unidad e incluso pueden llegar a evolucionar a polos reales que tienden a convertirse en dominantes. El efecto en la respuesta temporal (Figura 5.4) es de disminuir las oscilaciones e incrementar poco a poco el tiempo de establecimiento. El efecto el similar al descrito en el capítulo 3. También puede verse que cada horizonte de predicción tiene una fuerte influencia en la variable que gobierna, pero un efecto más limitado en la otra. Esto concuerda con el efecto desacoplador del DMC estudiado en el capítulo anterior.

\subsection{Influencia del horizonte de control}

En el Capítulo 2 se concluyó que el horizonte de control tenía una influencia limitada en los polos del sistema y, por lo tanto, en la respuesta temporal. Para comprobar si en multivariable esto se sigue cumpliendo, se va a realizar un experimento similar al de la sección anterior, variando M y manteniendo el resto de parámetros fijos con unos valores no demasiado elevados para minimizar su efecto $(\operatorname{Pr}=5$ y $\lambda=0,2)$.

Las Figuras 5.5 y 5.6 muestran la evolución de los polos en lazo cerrado conforme varía el horizonte de control. Dichas gráficas muestran que este parámetro tiene una escasa influencia en la evolución de los polos en lazo cerrado.

Las 5.7 y 5.8 muestran la evolución de la respuesta temporal al variar el horizonte de control, que apenas se ve afectada por este parámetro.

Estos resultados concuerdan con los obtenidos en el capítulo 3 y las referencias [59], [60], [52], [16] y [17]. En estos trabajos, se concluye que el horizonte de control tiene una influencia limitada en el la respuesta temporal. Aún así no debe descuidarse este parámetro y pueden darse una serie de reglas para su selección:

- Debe ser superior al número de modos inestables del sistema

- Su valor suele estar entre 2 y 6 [16] y [17]. Y la experiencia demuestra que un valor de 3 suele ser válido para la mayoría de ocasiones [52].

\subsection{Influencia del factor de peso}

Las simulaciones realizadas en el capítulo 3 indicaban que el factor de peso tendía a aumentar la parte imaginaria de los polos complejos a la vez que disminuía su parte real Esto causaba que los polos complejos perdieran su dominancia en favor de los polos reales, lo que limitaba las oscilaciones. Este es el comportamiento comúnmente observado cuando se incrementa este parámetro. En esta sección se va a realizar una serie de simulaciones 

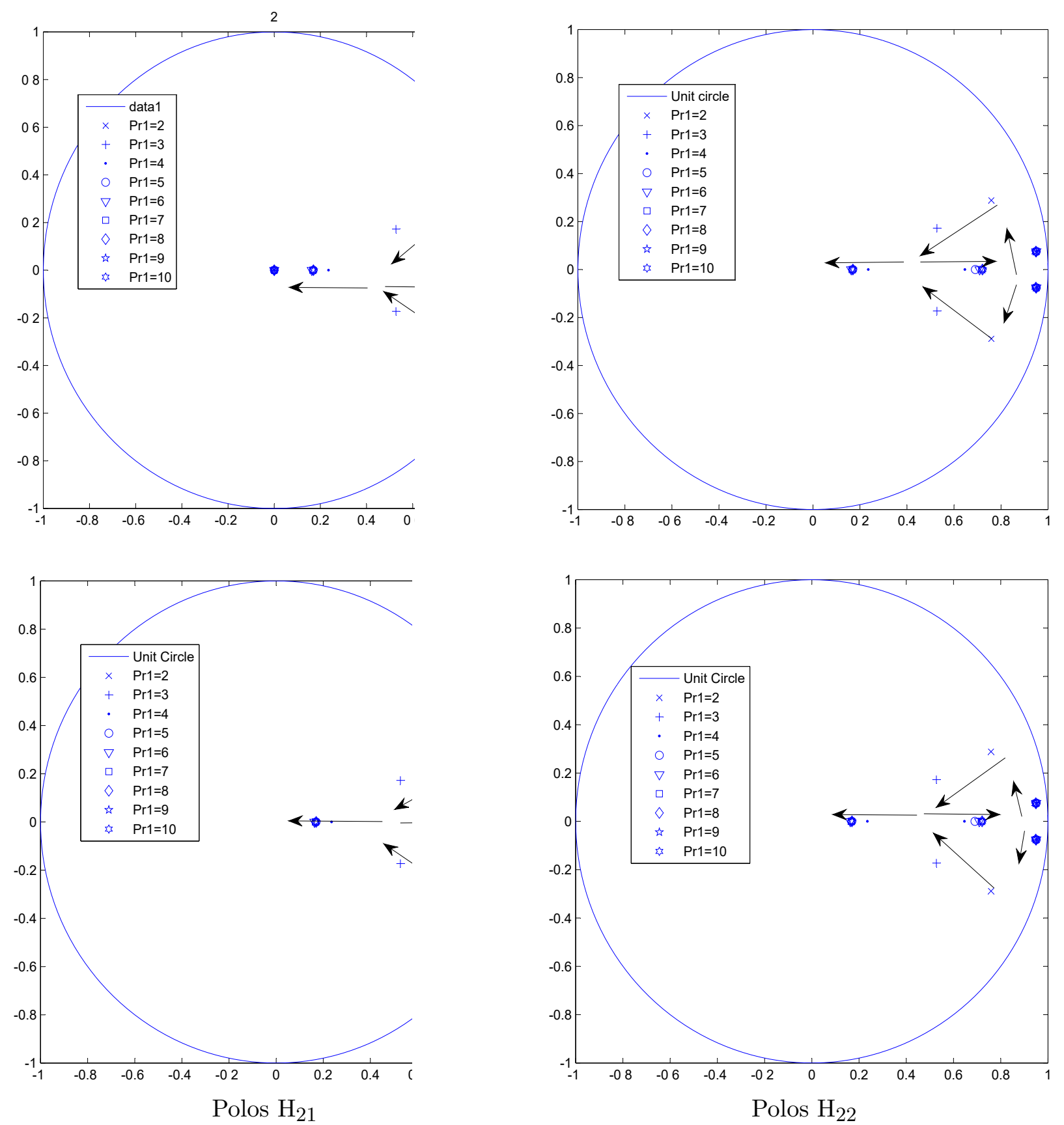

Figura 5.1: Evolución de los polos variando $\operatorname{Pr}_{1}$ para un valor fijo de $\operatorname{Pr}_{1}=2$ 

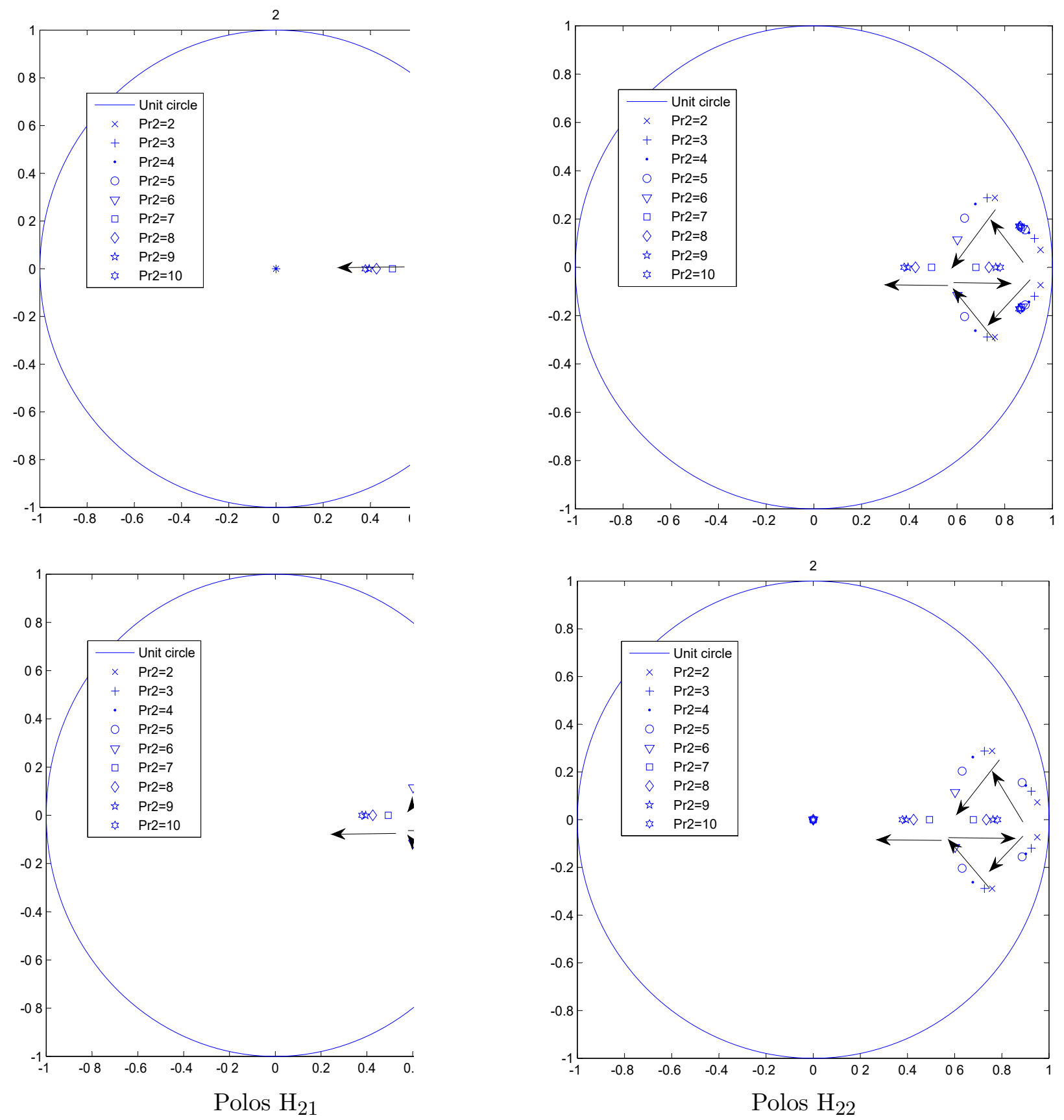

Figura 5.2: Evolución de los polos variando $\operatorname{Pr}_{2}$ para un valor fijo de $\operatorname{Pr}_{2}=2$ 


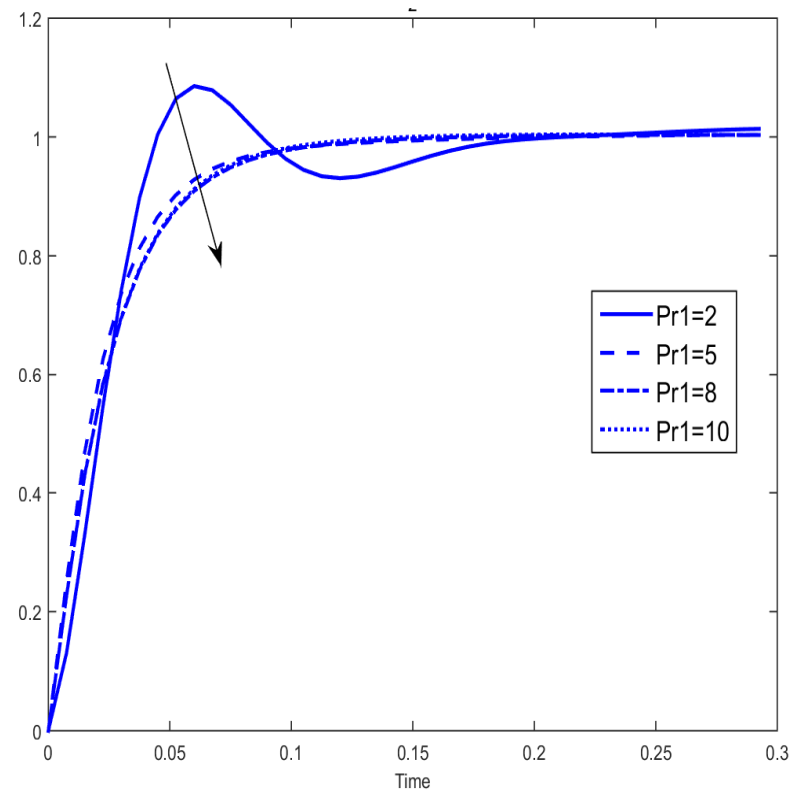

$\mathrm{y}_{1}$ variando $\operatorname{Pr}_{1}$

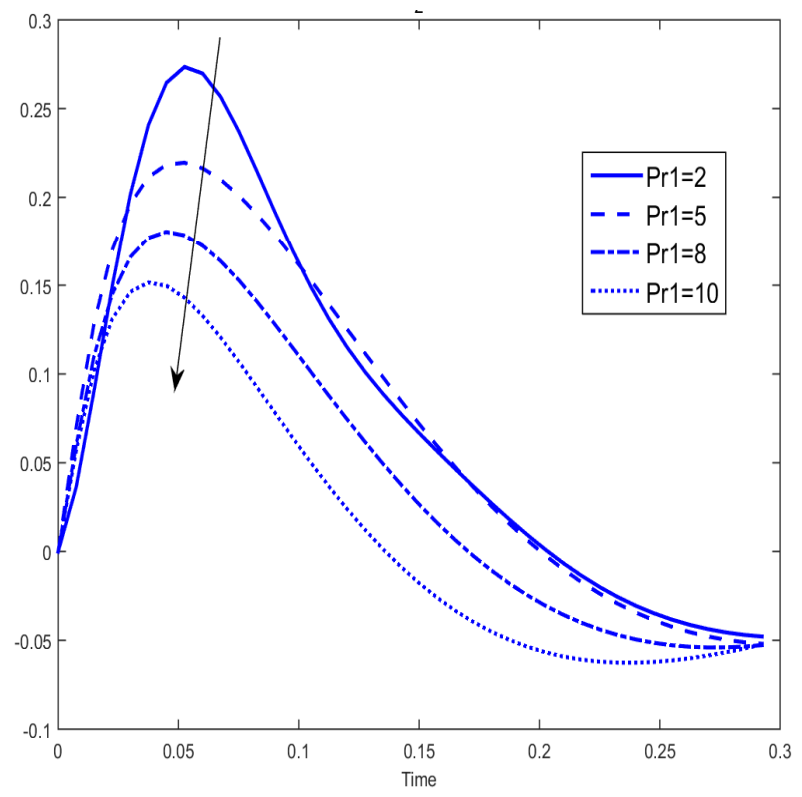

$\mathrm{y}_{2}$ variando $\operatorname{Pr}_{1}$

Figura 5.3: Evolución de la respuesta variando $\operatorname{Pr}_{1}$ para un valor fijo de $\operatorname{Pr}_{2}=2$

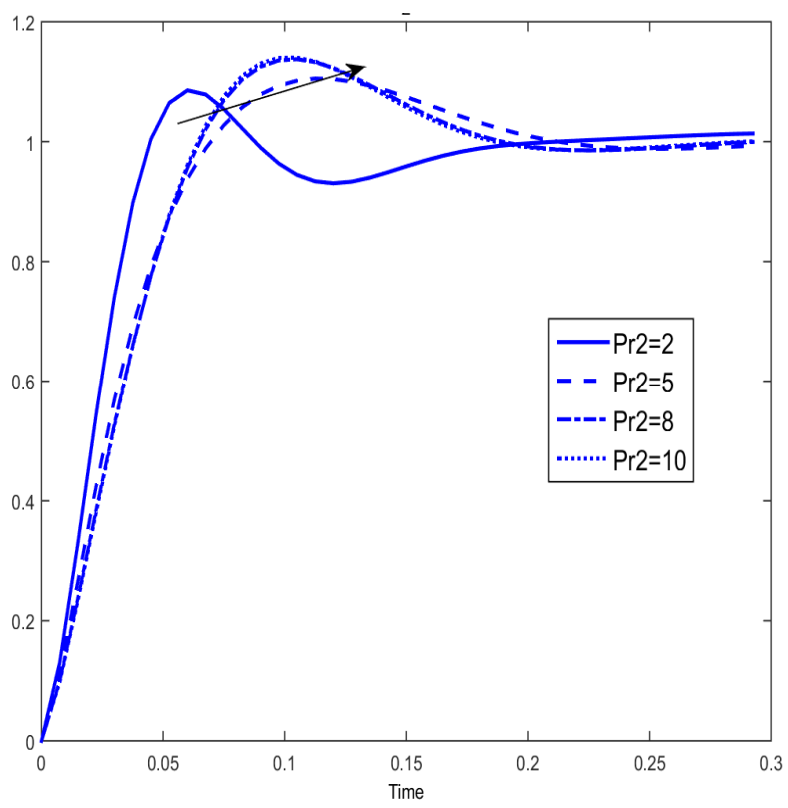

y 1 variando $\operatorname{Pr}_{2}$

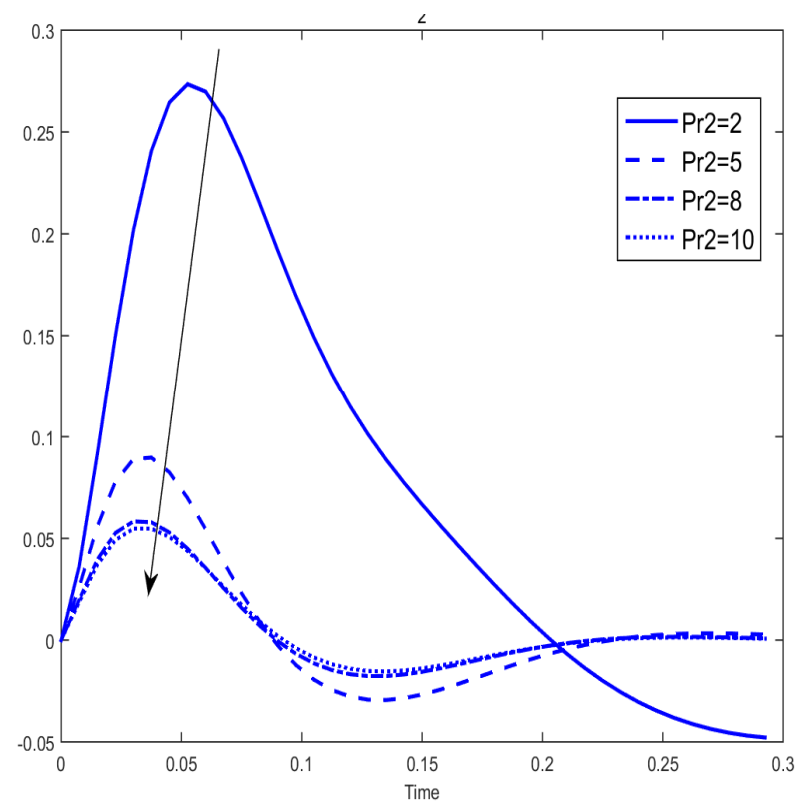

$\mathrm{y}_{2}$ variando $\mathrm{Pr}_{2}$

Figura 5.4: Evolución de la respuesta variando $\operatorname{Pr}_{2}$ para un valor fijo de $\operatorname{Pr}_{1}=2$ 

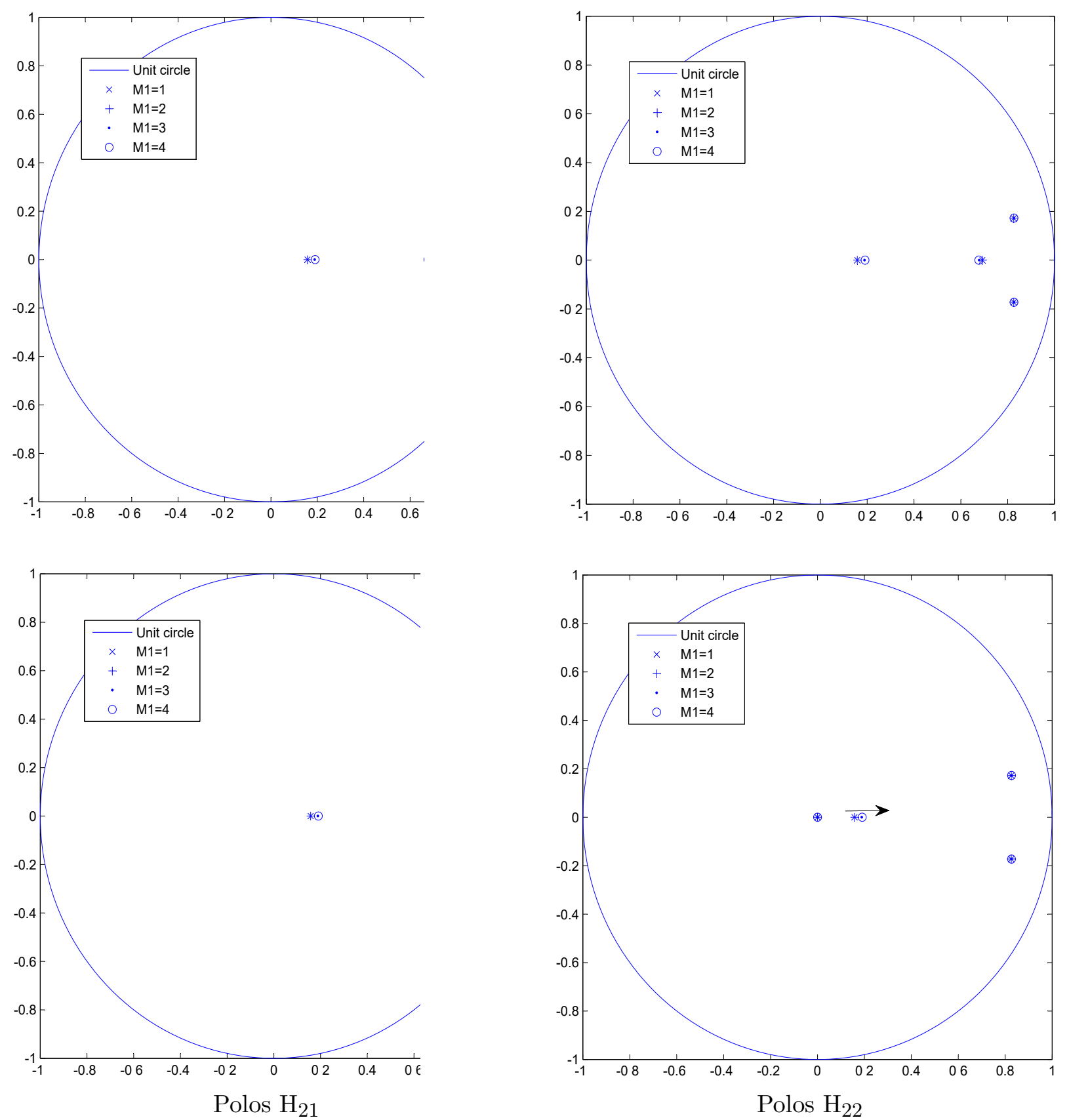

Figura 5.5: Evolución de los polos variando $\mathrm{M}_{1}$ para un valor fijo de $\mathrm{M}_{2}=1$ 

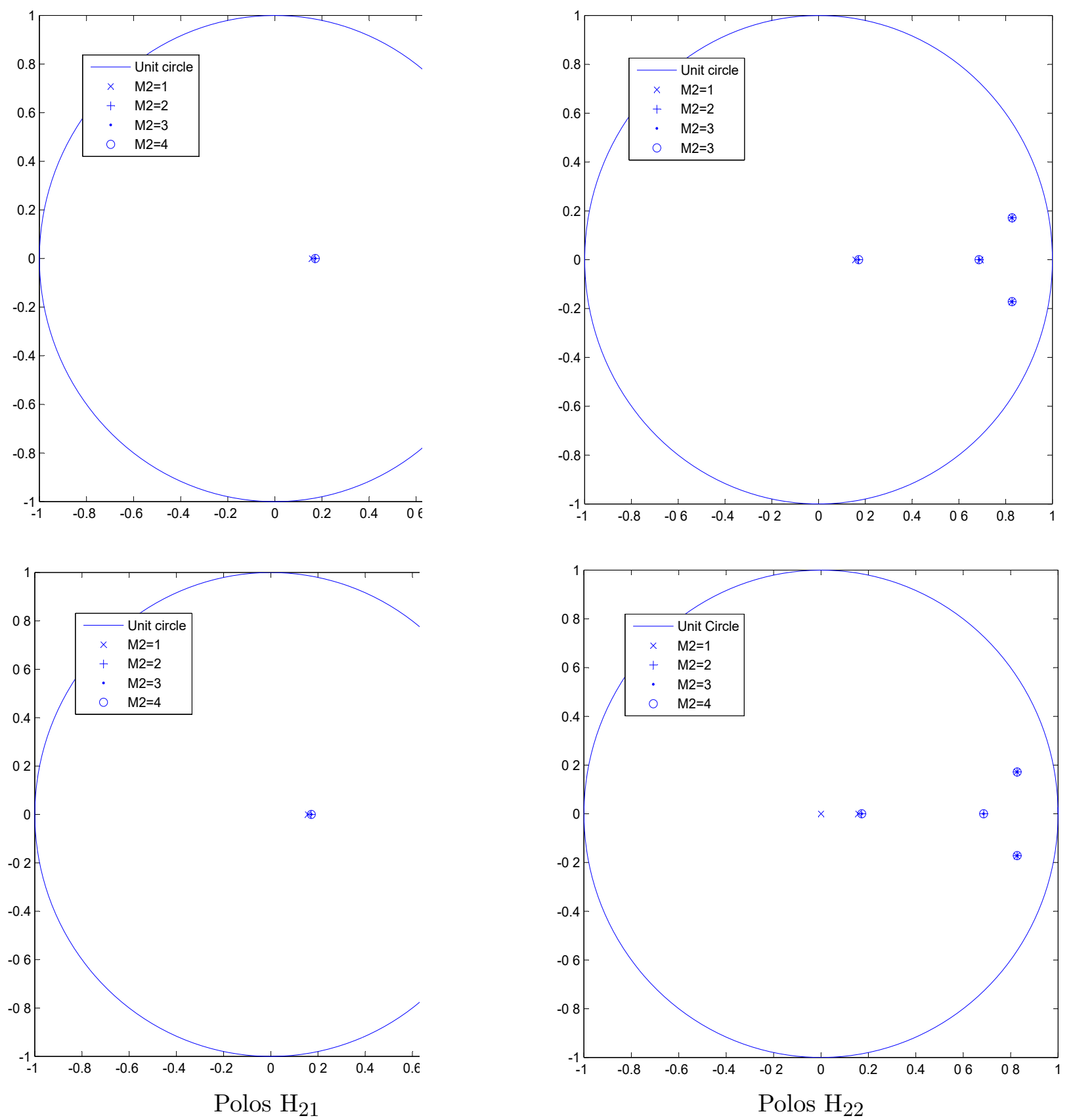

Figura 5.6: Evolución de los polos variando $\mathrm{M}_{2}$ para un valor fijo de $\mathrm{M}_{1}=1$ 

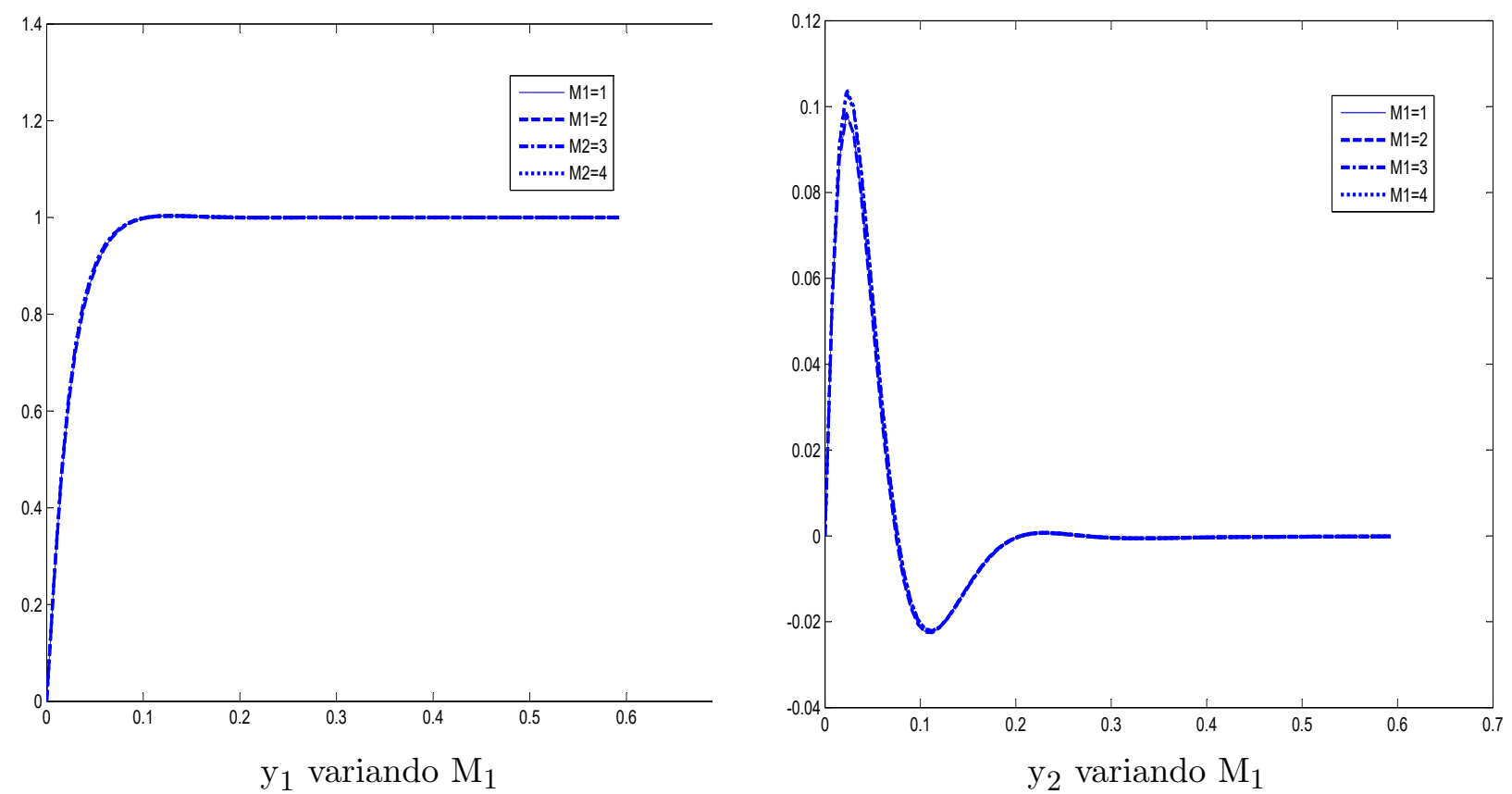

Figura 5.7: Evolución de la respuesta variando $\mathrm{M}_{1}$ para un valor fijo de $\mathrm{M}_{2}=1$

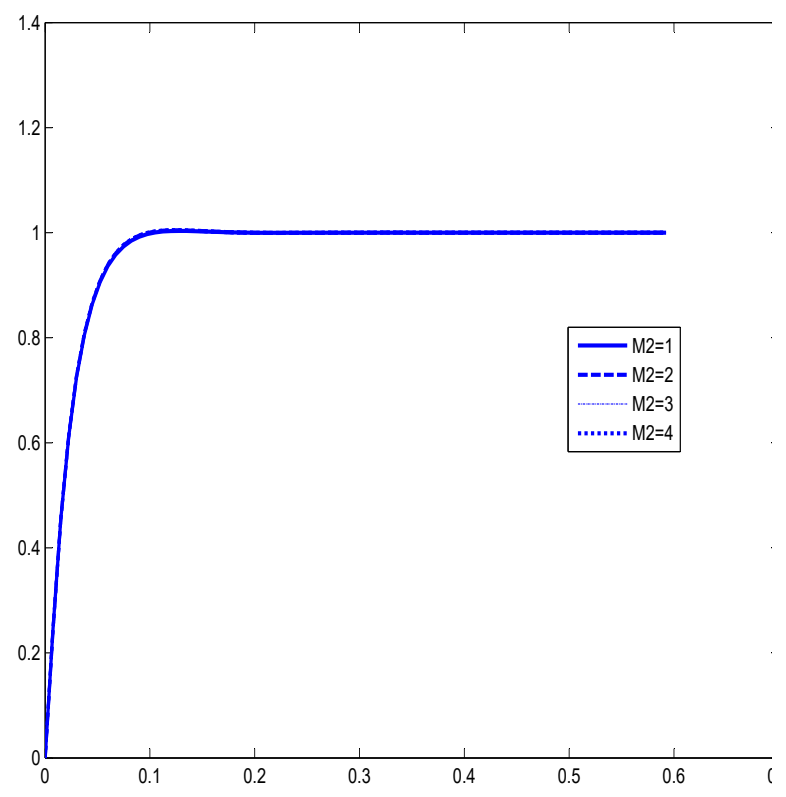

$\mathrm{y}_{1}$ variando $\mathrm{M}_{2}$

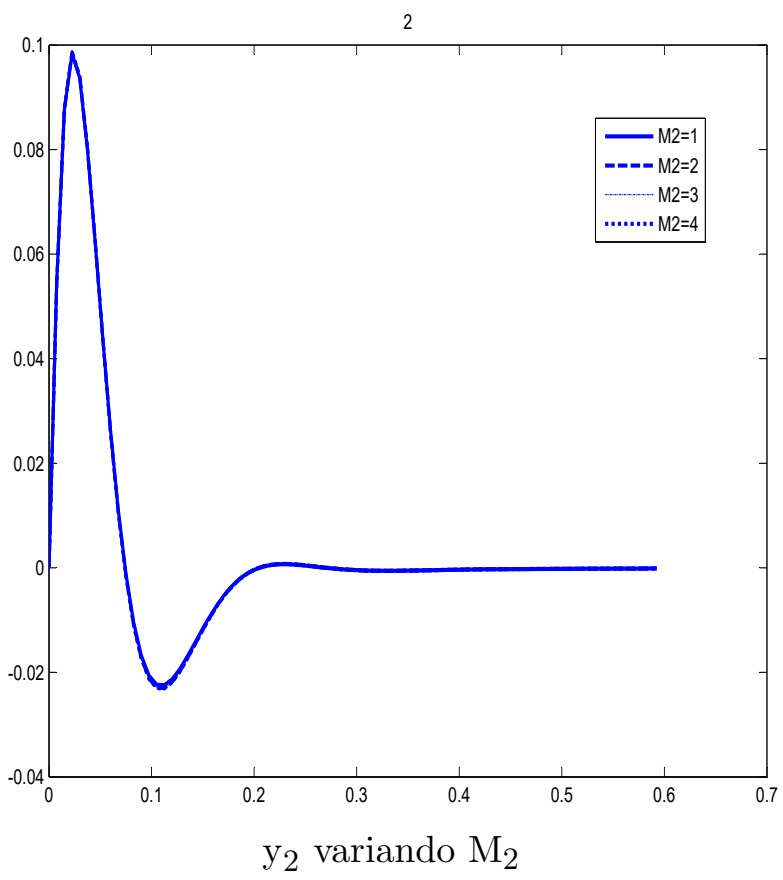

$\mathrm{y}_{2}$ variando $\mathrm{M}_{2}$

Figura 5.8: Evolución de la respuesta variando $\mathrm{M}_{2}$ para un valor fijo de $\mathrm{M}_{1}=1$ 
(similares a las de las secciones anteriores) en las que se mantendrán el resto de parámetros fijos $((\operatorname{Pr}=5$ y $\mathrm{M}=2))$ a la vez que se va variando el valor de los factores de peso para evaluar su efecto en los polos y la respuesta temporal.

$\mathrm{Al}$ igual que en capítulo 3 se construirán gráficas que relacionen el factor de peso con el primer incremento de control para acotar su zona de influencia. En monovariable bastaba una curva en dos dimensiones. En multivariable esta curva única evoluciona a:

- Una superficie en tres dimensiones, para conocer el efecto de cada factor de peso en una variable controlada.

- Una gráfica por cada variable controlada.

La Figura 5.9 muestran las superficies mencionadas, en las que puede apreciarse que cada variable manipulada solo se ve afectada por su factor de peso correspondiente.

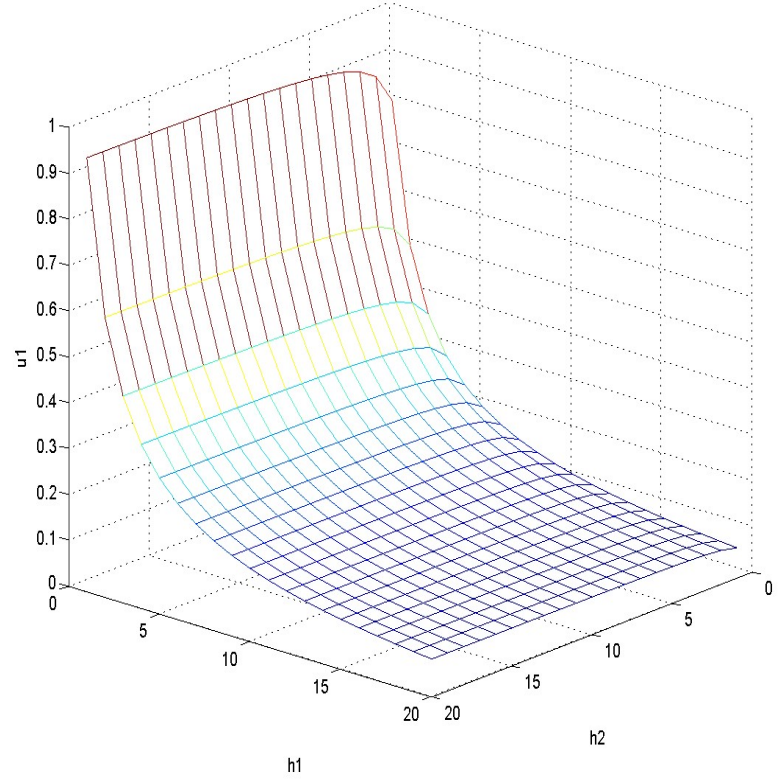

Superficie para $\mathrm{u}_{1}$

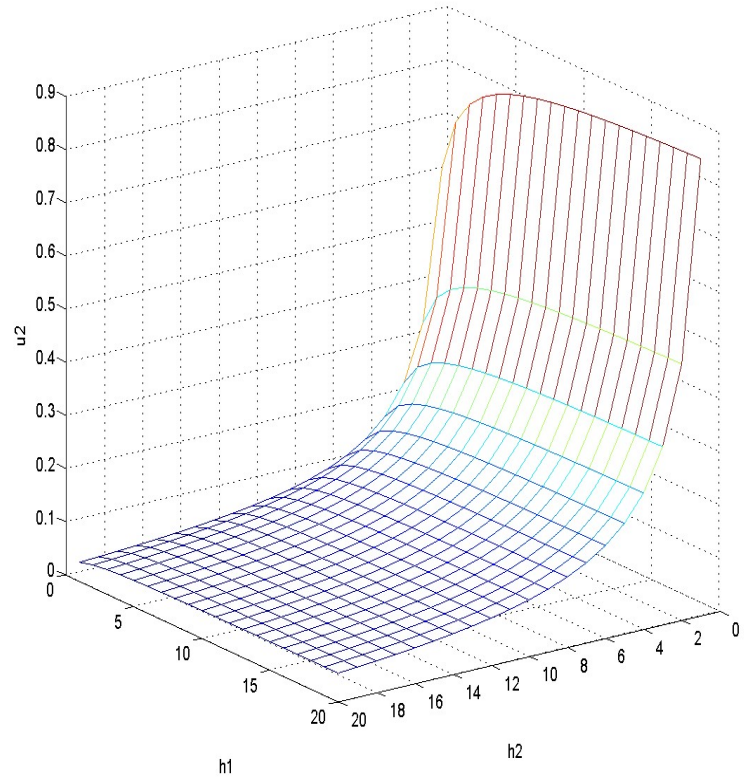

Superficie para $\mathrm{u}_{2}$

Figura 5.9: Evolución de los primeros incrementos de control con los factores de peso

Sin embargo, debe tenerse en cuenta que en el caso multivariable que cada variable controlada es la suma del efecto que tienen sobre ella cada variable manipulada (y sus factores de peso correspondientes). Para conocer el efecto que tienen los factores de peso, ya no basta con estudiar el primer incremento de control, sino que hay que obtener gráficas similares para las variable controladas (Figura 5.10).

Las Figuras 5.9 y5.10 dan una idea del efecto que tienen los factores de peso en las señales de control y las salidas:

- La Figura 5.9 muestra, como era de esperar debido al efecto desacoplador, que cada variable manipulada es afectada principalmente por su factor de peso correspondiente. Además permite obtener cual es el rango de valores útiles. 


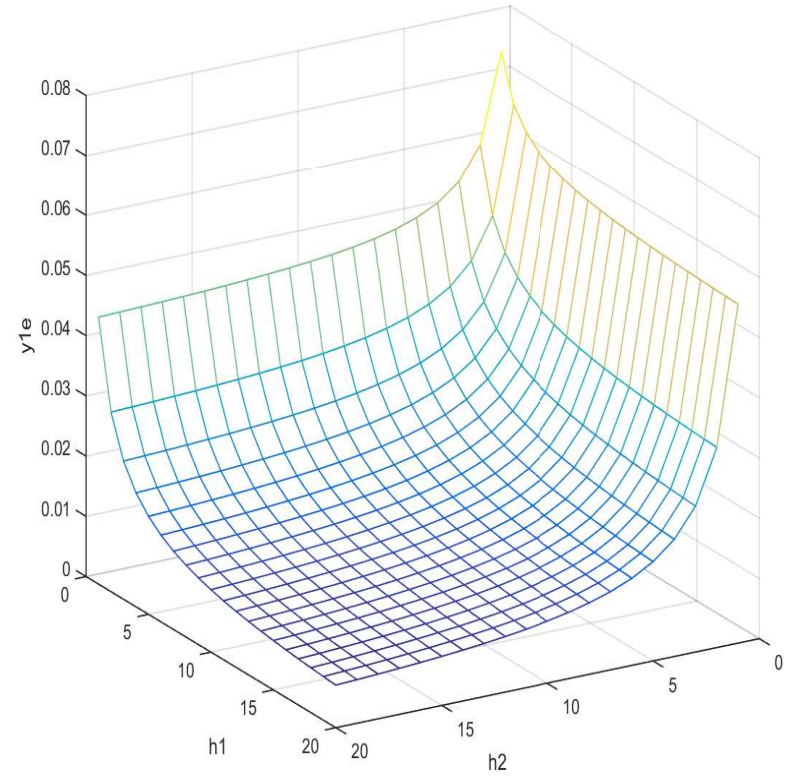

Superficie para $\mathrm{y}_{1}$

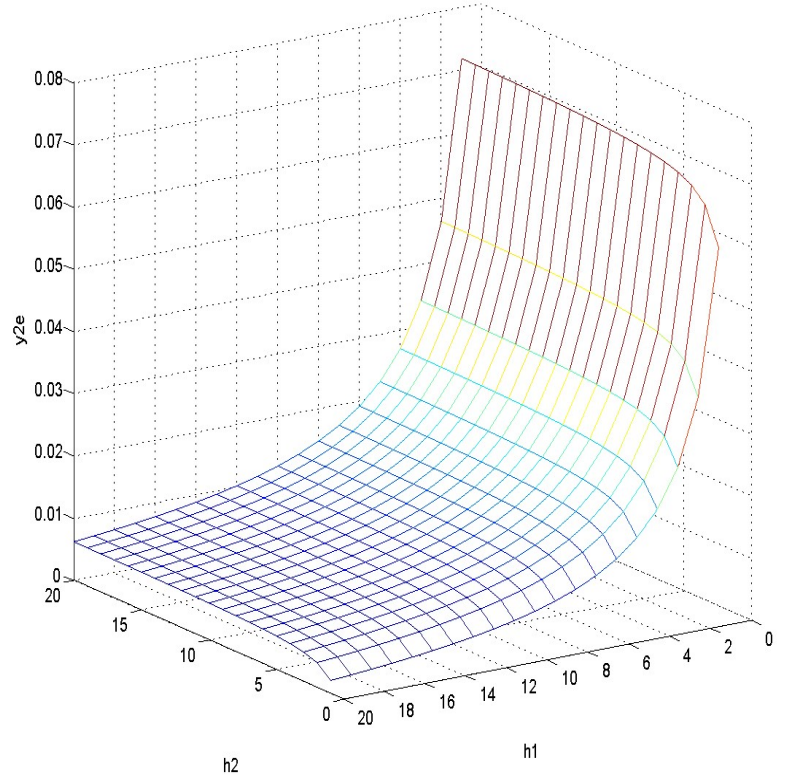

Superficie para $\mathrm{y}_{2}$

Figura 5.10: Evolución de los primeros valores de salida con los factores de peso

- La Figura 5.10 muestra que una variable controlada si puede depender de los dos factores de peso, así como los rangos útiles de para cada factor de peso. Esto es debido a que en un sistema multivariable, cada variable controlada viene dada por la suma de las contribución de cada variable manipulada (y cada variable controlada está gobernada por su factor de peso). En este caso $\mathrm{y}_{1}=\mathrm{H}_{11} \mathrm{u}_{1}+\mathrm{H}_{12} \mathrm{u}_{2}$.

En el capítulo 3 se ha estudiado el efecto del factor de peso para el caso monovariable. En sistemas SISO el factor de peso tiende a hacer más lentos los polos dominantes y a disminuir la parte imaginaria de los polos complejos. Esto afecta a la respuesta temporal disminuyendo las oscilaciones a cambio de aumentar el tiempo de establecimiento. Sin embargo, el sistemas MIMO el efecto del factor de peso no es tan simple debido a los acoplamientos.Las Figuras 5.11 y 5.12 muestran que la evolución de los polos es diferente a lo esperado. Al aumentar los factores de peso se aumenta la parte imaginaria de los polos complejos e incluso se llegan a hacer dominantes. Por otra parte, en las figuras 5.13 y 5.14 puede verse la evolución de la respuesta temporal. Al aumentar los factores de pesos llegan a aumentar las oscilaciones en la respuesta, un comportamiento contrario al habitual para este parámetro [52]

Incluso puede verse que, contrariamente a lo sucedido en monovariable, al aumentar uno de los factores de peso, una de las salidas aumenta su valor de sobrepico. Para entender porque este sistema funciona de forma inversa a lo que se esperaba, es necesario acudir a los valores de las variables manipuladas. La Figura 5.15 muestra como al aumentar un factor de peso (tanto $\lambda_{1}$ como $\lambda_{2}$ ), la variable manipulada que gobierna empieza a 

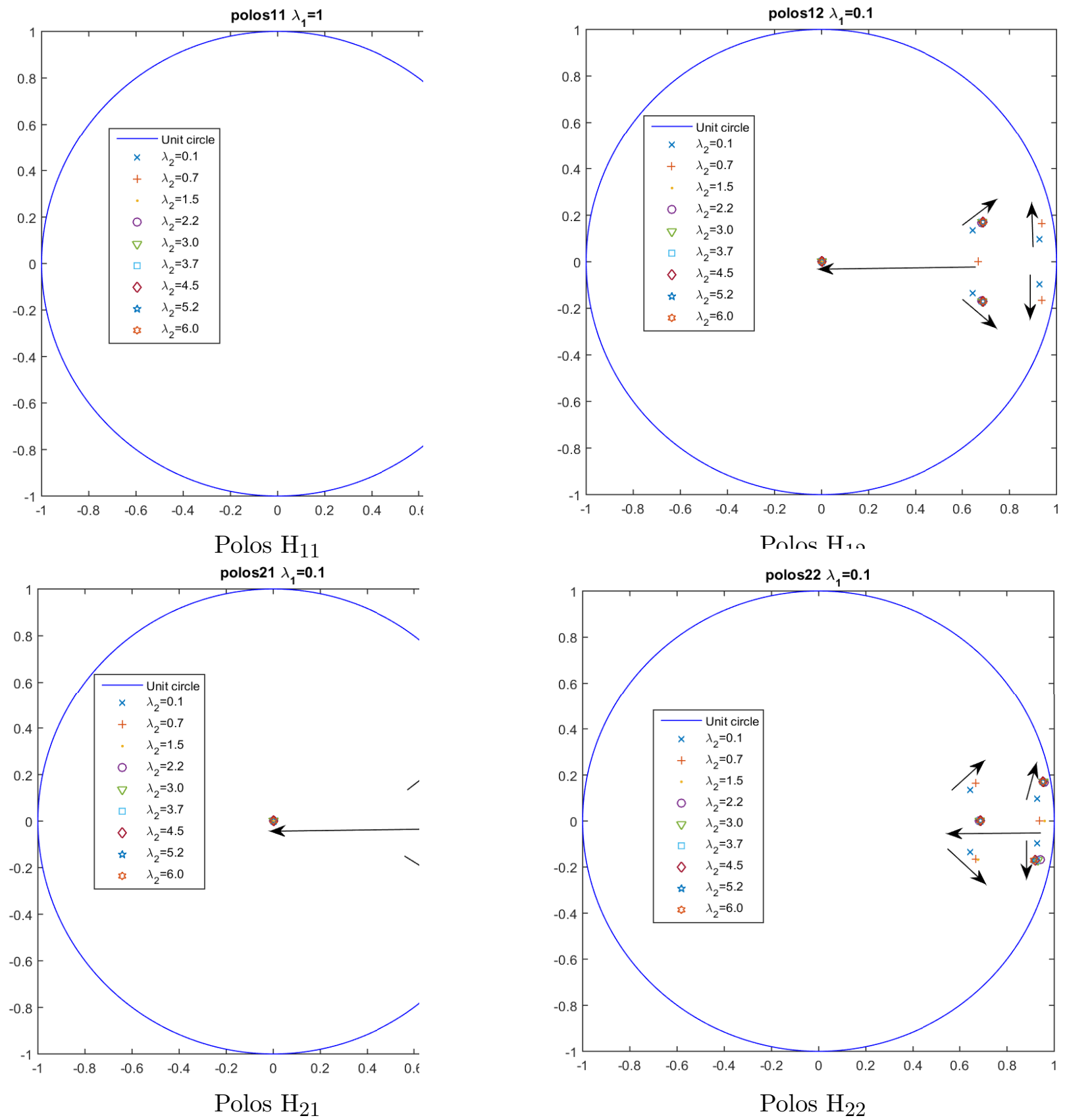

Figura 5.11: Evolución de los polos variando $\lambda_{2}$ para un valor fijo de $\lambda_{1}=0,1$ 

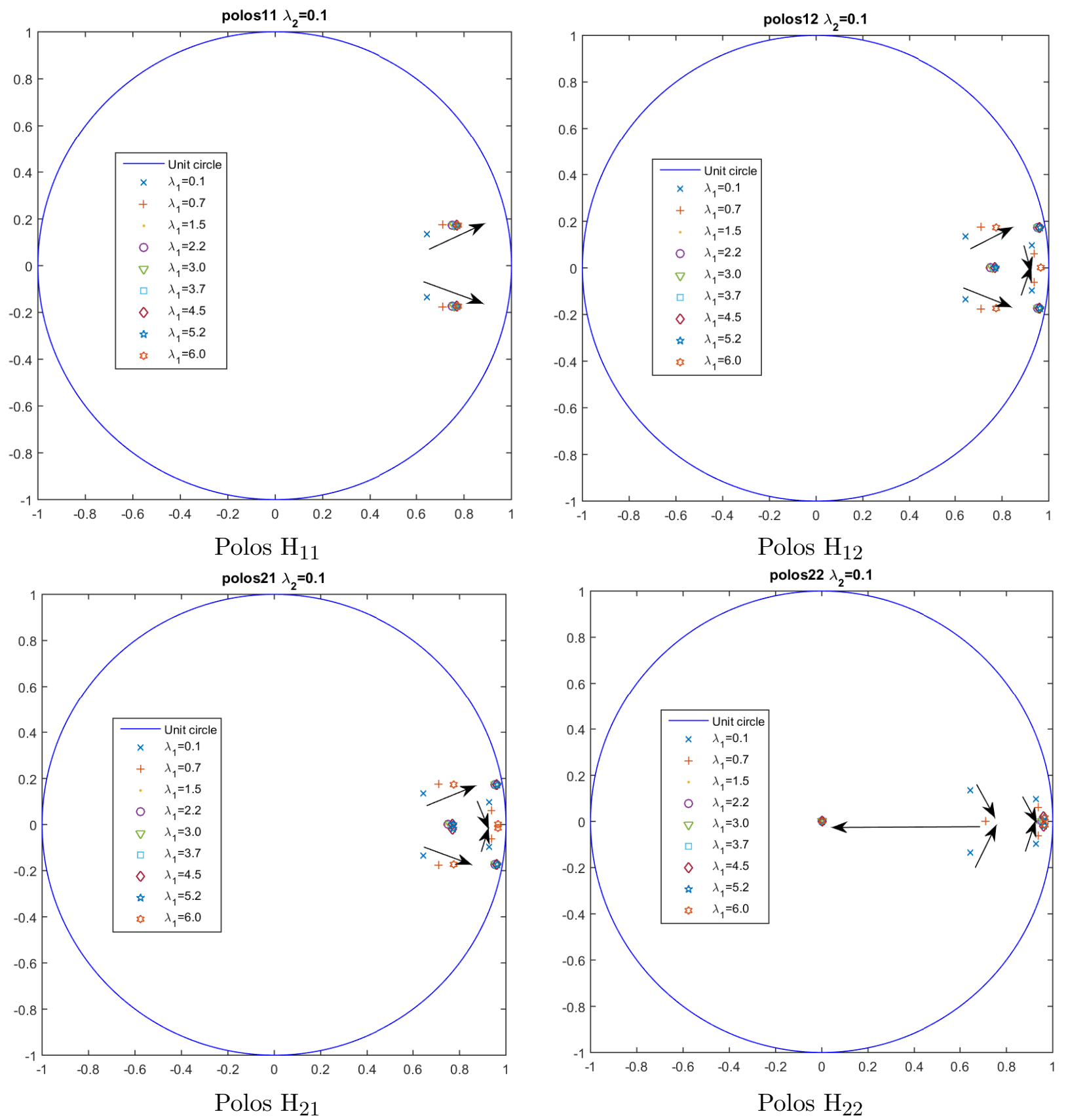

Figura 5.12: Evolución de los polos variando $\lambda_{1}$ para un valor fijo de $\lambda_{2}=0,1$ 


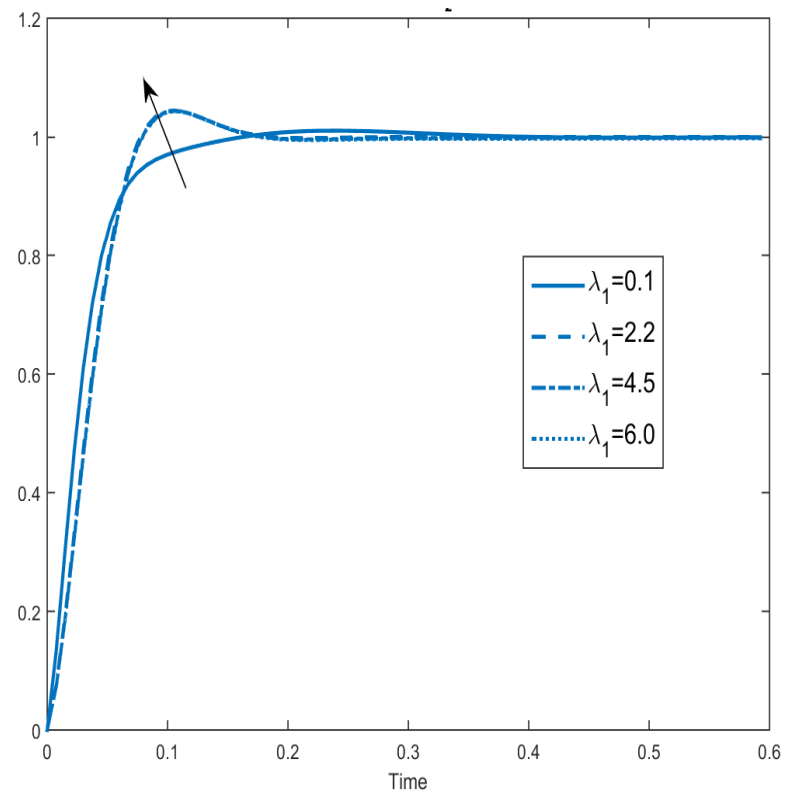

$\mathrm{y}_{1}$ variando $\lambda_{1}$

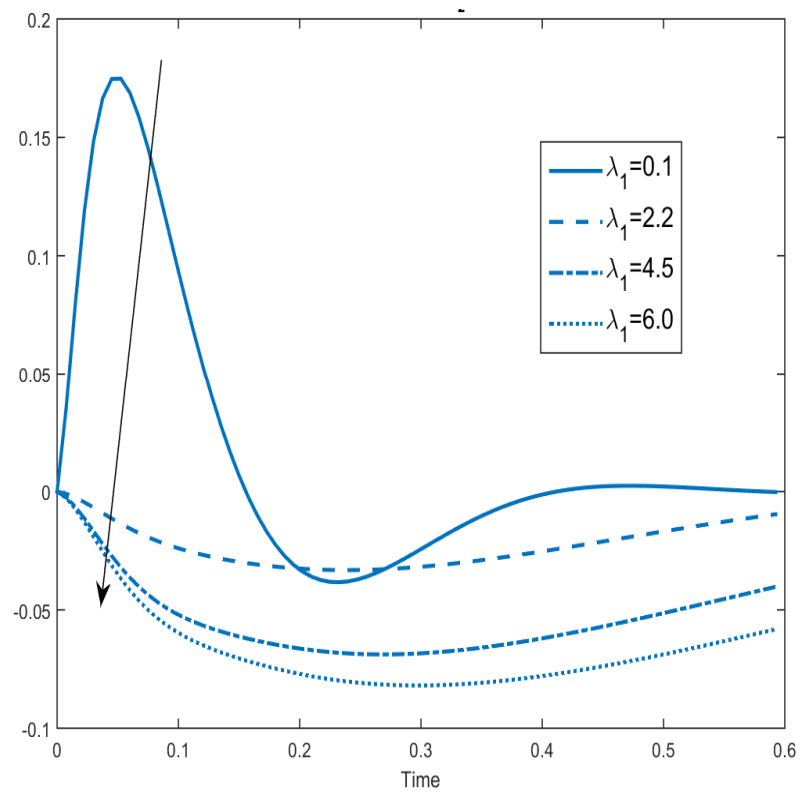

$\mathrm{y}_{2}$ variando $\lambda_{1}$

Figura 5.13: Evolución de la respuesta variando $\lambda_{1}$ para un valor fijo de $\lambda_{2}=0,1$

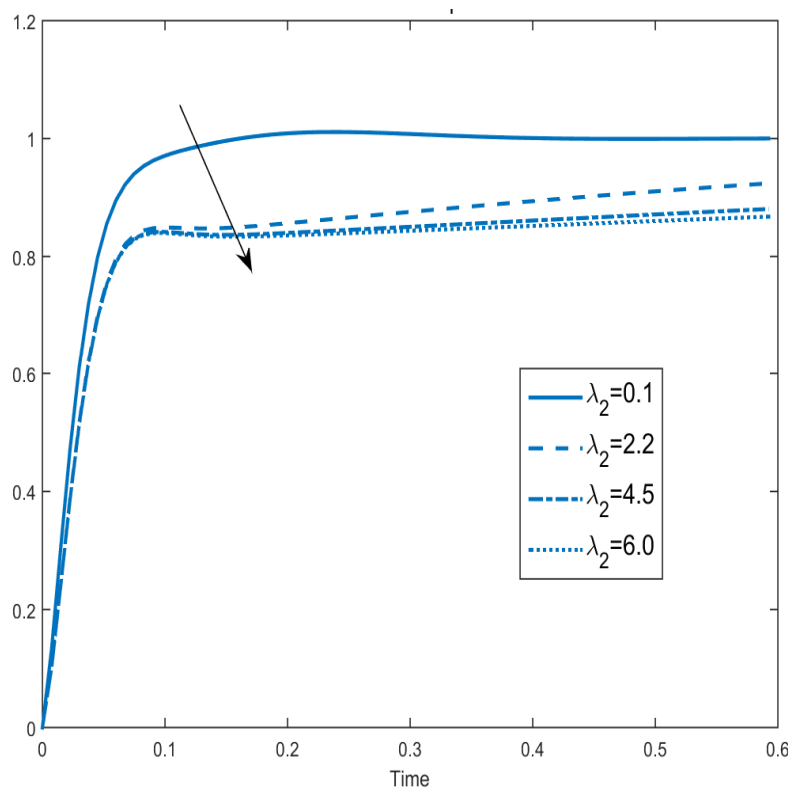

$\mathrm{y}_{1}$ variando $\lambda_{2}$

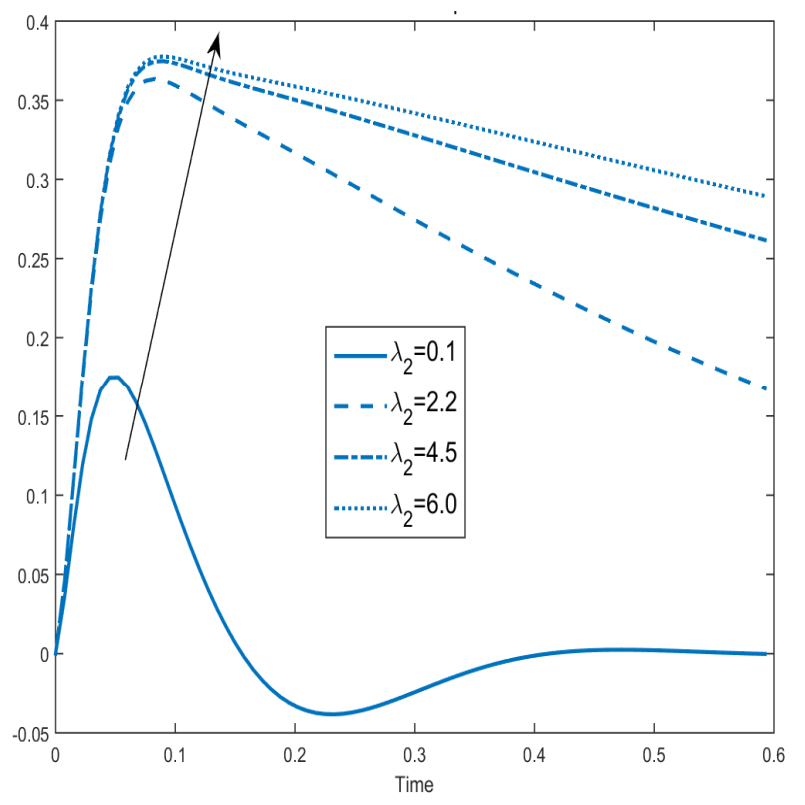

$\mathrm{y}_{2}$ variando $\lambda_{2}$

Figura 5.14: Evolución de la respuesta variando $\lambda_{2}$ para un valor fijo de $\lambda_{1}=0,1$ 
disminuir a la vez que una de las variable controladas aumenta su sobrepico. El motivo de esto es que al limitar el valor de una variable controlada, solo queda otra para cumplir con los objetivos de control. Por ejemplo, si se aumenta $\lambda_{1}$ comenzará a disminuir $u_{1}$. Esto obligará al controlador a aumentar el valor de $u_{2}$ para cumplir con los objetivos de control. Sin embargo, aumentar demasiado el valor de $\mathrm{u}_{2}$, hará que el valor de sobrepico de $\mathrm{y}_{2}$ crezca.

Para calcular correctamente el valor de los factores de peso será necesario tener el cuenta la matriz de ganancias relativas (Relative Gain Array, RGA) del sistema a controlar.

$$
\mathbf{R G A}=\left(\begin{array}{ll}
0,283 & 0,717 \\
0,717 & 0,283
\end{array}\right)
$$

Como se ha visto en el capítulo 4, cada elemento de la RGA representa la relación entre una variable controlada y una variable manipulada. Para el motor alternador, el valor de todos los elementos está entre 0 y 1 . Esto significa que la ganancia aumenta en lazo cerrado debido a las interacciones. Dependiendo de cada lazo, la influencia de las interacciones pueden ser de mayor o menor importancia que la vía principal.

- $\mathrm{RGA}_{11}=0,283$ implica que $\mathrm{u}_{1}$ tiene menos influencia en $\mathrm{y}_{1}$ que $\mathrm{u}_{2}$.

- $\operatorname{RGA}_{12}=0,717$ implica que $\mathrm{u}_{1}$ tiene más influencia en $\mathrm{y}_{2}$ que $\mathrm{u}_{2}$.

- $\mathrm{RGA}_{21}=0,717$ implica que $\mathrm{u}_{2}$ tiene más influencia en $\mathrm{y}_{1}$ que $\mathrm{u}_{1}$.

- $\mathrm{RGA}_{22}=0,283$ implica que $\mathrm{u}_{2}$ tiene menos influencia en $\mathrm{y}_{2}$ que $\mathrm{u}_{1}$.

El estudio de la matriz RGA revela que:

1. Las interacciones en este sistema son fuertes. Ambas variables manipuladas influyen sensiblemente en cada variable controlada.

2. Cada variable manipulada tiene un efecto fuerte en una variable controlada y un efecto menor en la otra. Sin embargo la magnitud de este efecto es la misma para ambas variables. Esto queda evidenciado por el hecho de la matriz es simétrica.

Estas conclusiones indican que $\mathrm{u}_{1}$ tiene más influencia en $\mathrm{y}_{2}$ que $\mathrm{u}_{2} \mathrm{y}$ viceversa. Si el valor de $u_{1}$ se limita en exceso, el DMC solo tendrá $u_{2}$ para controlar $\mathrm{y}_{2}$. Pero esta variable manipulada tiene menos influencia que $\mathrm{u}_{1}$, lo que obliga al DMC a dar valores cada vez más altos para $\mathrm{u}_{2}$ conforme $\lambda_{1}$ aumenta para conseguir controlar $\mathrm{y}_{2}$. Estos valores elevados de $\mathrm{u}_{2}$ influyen negativamente en el control de $\mathrm{y}_{2}$, que es la variable controlada sobre la que tiene más influencia.

Por lo tanto, en sistemas MIMO, a la hora de seleccionar los factores de peso es necesario tener en cuenta no solo su rango de influencia, sino también su efecto en cada variable controlada. Como se ha visto antes, cada variable manipulada tiene un efecto fuerte en una variable controlada y un efecto menor en la otra.Y la magnitud de este efecto es la misma para ambas variables. En este caso no se puede penalizar una variable manipulada por encima de otra. Los dos factores de peso deben coincidir. 

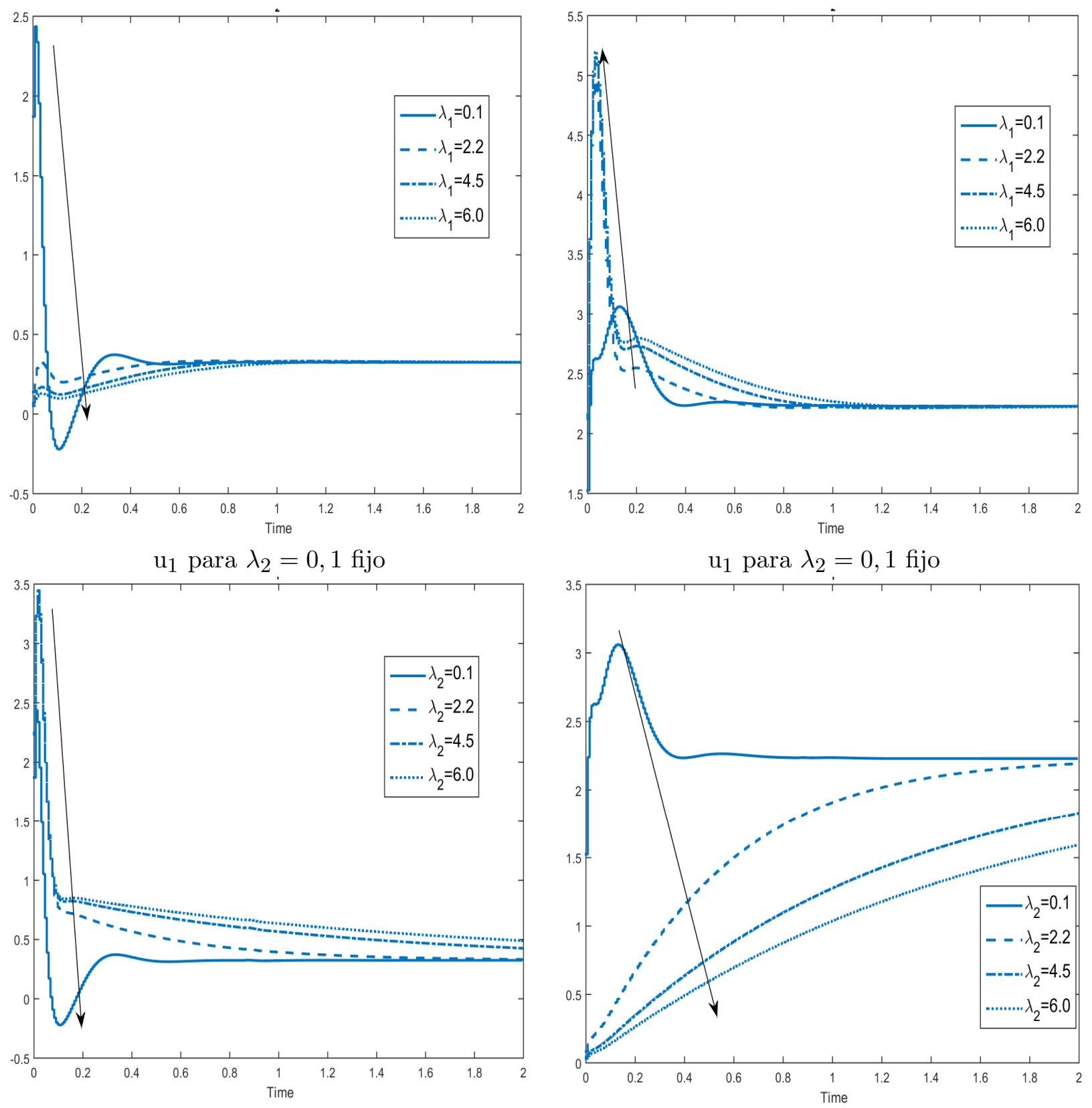

$\mathrm{u}_{1}$ para $\lambda_{1}=0,1$ fijo

$\mathrm{u}_{2}$ para $\lambda_{1}=0,1$ fijo

Figura 5.15: Evolución de $\mathrm{u}_{1} \mathrm{y} \mathrm{u}_{2}$ variando $\lambda_{1} \mathrm{y} \lambda_{2}$ 


\subsection{Reglas de diseño para sistemas MIMO}

Según lo visto en las secciones anteriores, se pueden obtener la siguientes conclusiones:

- EL horizonte predicción actúa de manera similar a como funciona en monovariable. Es decir, tiende a hacer que los polos reales se conviertan en dominantes. A mayor horizonte de predicción los polos reales se acercan cada vez más al círculo unidad. Las oscilaciones desaparecen, pero el tiempo de establecimiento aumenta.

- El horizonte de control tiene una influencia limitada, tal y como sucede en monovariable.

- El factor de peso es el más afectado por el paso a multivariable. Las interacciones hacen que parte de las lecciones aprendidas en capítulos anteriores y en la literatura se deban reconsiderar en MIMO. Ahora es necesario tener en cuenta las interacciones a la hora de calcular el factor de peso. Para ello, aparte de las gráficas del primer incremento de control, que deben calcularse y estudiarse a la vez para todas las variables, es necesario calcular la matriz RGA del sistema para saber hasta que punto pueden diferir entre si los distintos factores de peso.

Teniendo en cuenta lo anterior y lo expuesto en el capítulo 3, se pueden escribir las siguientes reglas de diseño para sistemas MIMO:

1. $\mathrm{n}_{\mathrm{gi}}=\operatorname{Pr}_{\mathrm{i}}+\mathrm{n}_{\mathrm{i}}$, donde $\mathrm{n}$ es el número requerido de valores $\mathrm{g}$ necesarios para alcanzar el estacionario en el modelo de escalón unitario. En MIMO será necesario calcular este número para cada una de las funciones de la matriz de transferencia y seleccionar el valor más alto. Es decir, $\mathrm{n}_{\mathrm{g}}=\max \left(\mathrm{Pr}_{\mathrm{i}}+\mathrm{n}_{\mathrm{i}}\right)$

2. $\operatorname{Pr}=\tau_{\mathrm{p}}$, siendo $\tau_{\mathrm{p}}$ la constante de tiempo del equivalente FOPDT (tiempo para alcanzar el $63 \%$ del valor del escalón). Se seleccionará el valor más alta para cada variable manipulada, $\operatorname{Pr}_{\mathrm{j}}=\max \left(\operatorname{Pr}_{1 \mathrm{j}} \ldots \mathrm{Pr}_{\mathrm{ij}}\right)$, siendo i el número de variables controladas.

3. M tiene una influencia limitada en el proceso para valores pequeños de $\operatorname{Pr}$ (inferiores a $\tau_{\mathrm{p}}$ ) pero corrige el comportamiento como lazo abierto cuando Pr es demasiado alto (mayor a $\tau_{\mathrm{p}}$ ). También, según Rawlings y Muske [51], este valor debería ser mayor o igual al número de modos inestables del sistema para garantizar la estabilidad. Un valor de $\mathrm{M}=3$ ha demostrado dar un rendimiento cercano al óptimo, [52].

4. Para calcular el tiempo de muestreo se han tenido en cuenta las consideraciones de Shridhar y Cooper [16] [17], que proponen tomar el máximo entre $0.1 \tau_{\mathrm{p}}$ y $0.5 \theta$ del equivalente FOPDT correspondiente. Esto ha demostrado dar resultados precisos. Este valor se considerará un máximo. Se seleccionará el valor mínimo de aplicar esta regla a todos los elementos de la matriz de transferencia.

5. En sistemas multivariable los factores de peso no pueden seleccionarse independientemente, sino que deben seleccionarse de tal forma que no se elimine ningún grado 
de libertad necesario para cumplir con los objetivos de control. Para ello, aparte de las curvas de primer incremento de control, será necesario estudiar la influencia de cada variable manipulada en las variables controladas mediante la matriz RGA.

\subsection{Validación de reglas de diseño para sistemas MIMO}

Al igual que en capítulo 3, se van a validar las reglas de diseño sintonizando varios benchmark extraídos de la literatura. Los benchmark seleccionados son los mismos que los usados en el capítulo 4 para verificar la validez de la formulación LTI. Además se ajustará el controlador DMC para la columna de destilación de alta pureza descrita en el apéndice A de [56]. Este modelo dinámico es más cercano a un sistema físico real que los benchmark representados directamente con una función de transferencia.

\subsubsection{Benchmark 1}

Columna de destilación de Wood Berry, [54].

$$
\mathbf{G}=\left(\begin{array}{cc}
\frac{12.8 \mathrm{e}^{-\mathrm{s}}}{(16.7 \mathrm{~s}+1)} & \frac{-18.9 \mathrm{e}^{-3 \mathrm{~s}}}{(21 \mathrm{~s}+1)} \\
\frac{6.6 \mathrm{e}^{-7 \mathrm{~s}}}{(10.9 \mathrm{~s}+1)} & \frac{-19.4 \mathrm{e}^{-3 \mathrm{~s}}}{(14.4 \mathrm{~s}+1)}
\end{array}\right)
$$

La tabla 5.1 muestra los parámetros obtenidos aplicando las reglas de diseño enunciadas en la sección anterior, excepto el factor de peso. Para el factor de peso será necesario calcular las curvas del primer incremento de control, salida y matriz RGA.

\begin{tabular}{|c|c|c|c|c|c|c|c|}
\hline $\begin{array}{c}\text { Función } \\
\text { transferencia }\end{array}$ & $\begin{array}{c}\text { Constante } \\
\text { de tiempo }\end{array}$ & $\begin{array}{c}\text { Tiempo de } \\
\text { establecimiento }\end{array}$ & Retraso & $\begin{array}{c}\text { Tiempo de } \\
\text { muestreo }\end{array}$ & Pr & $\mathrm{M}$ & $\mathrm{n}_{\mathrm{g}}$ \\
\hline $\mathrm{G}_{11}$ & 16,7 & 100 & 1 & 1 & 20 & 1 & 120 \\
\hline $\mathrm{G}_{12}$ & 21 & 120 & 3 & 2 & 25 & 1 & 145 \\
\hline $\mathrm{G}_{21}$ & 10,9 & 80 & 7 & 1 & 20 & 1 & 100 \\
\hline $\mathrm{G}_{22}$ & 14,4 & 100 & 3 & 1 & 25 & 1 & 125 \\
\hline
\end{tabular}

Cuadro 5.1: Resultados del ajuste para benchmark 1

$$
\mathbf{R G A}=\left(\begin{array}{cc}
2 & -1 \\
-1 & 2
\end{array}\right)
$$

Atendiendo a la Figura 5.16 (en este caso la gráfica para la variable controlada es cero debido al retraso) el valor útil límite para $\lambda$ es 60 para ambas variables. Teniendo en cuenta la matriz RGA para este sistema, no es aconsejable que los factores de peso tomen valores diferentes. Por lo tanto, los valores para los parámetros del DMC son:

- $\operatorname{Pr}_{1}=\max \left(\operatorname{Pr}_{11}, \operatorname{Pr}_{21}\right)=17$

- $\operatorname{Pr}_{2}=\max \left(\operatorname{Pr}_{21}, \operatorname{Pr}_{22}\right)=21$ 


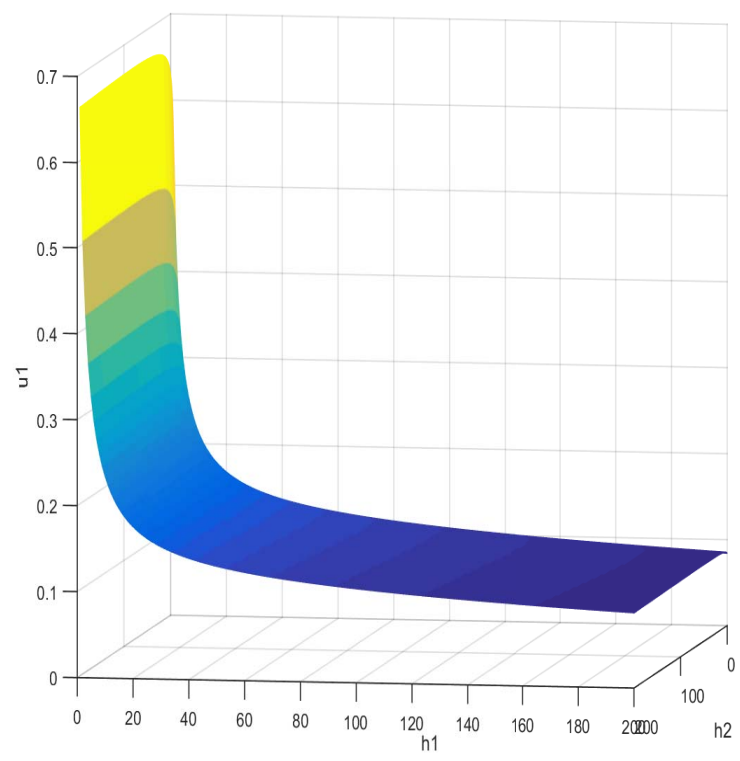

$u_{1}$ para $\lambda_{1}$ fijo

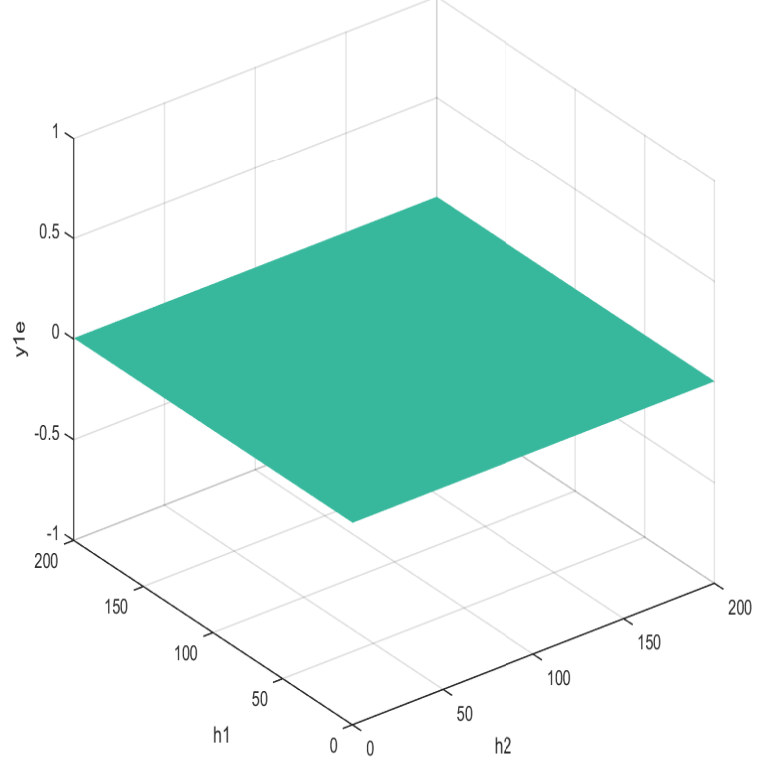

$\mathrm{y}_{1}$ para $\lambda_{1}$ fijo

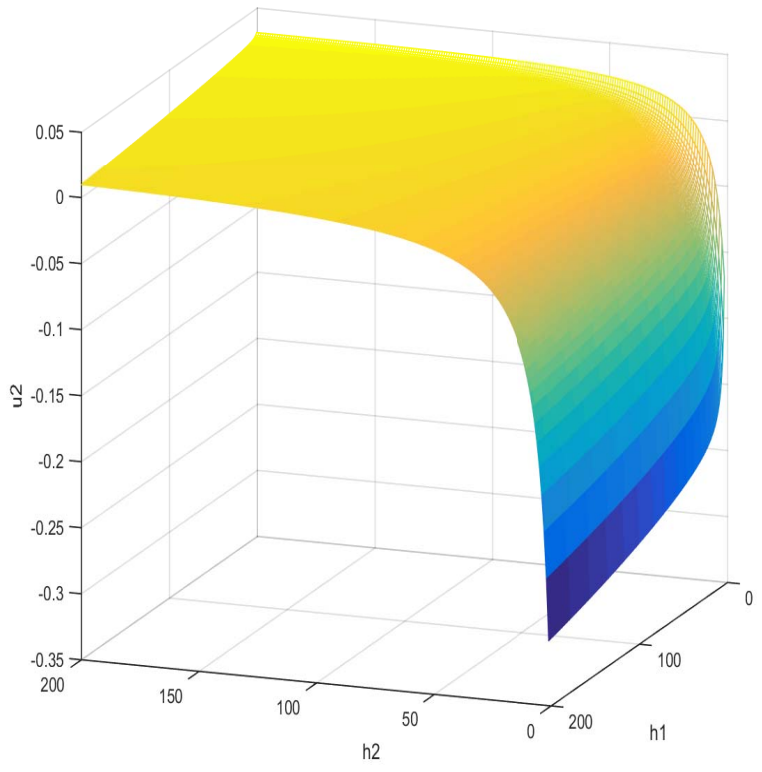

$u_{2}$ para $\lambda_{2}$ fijo

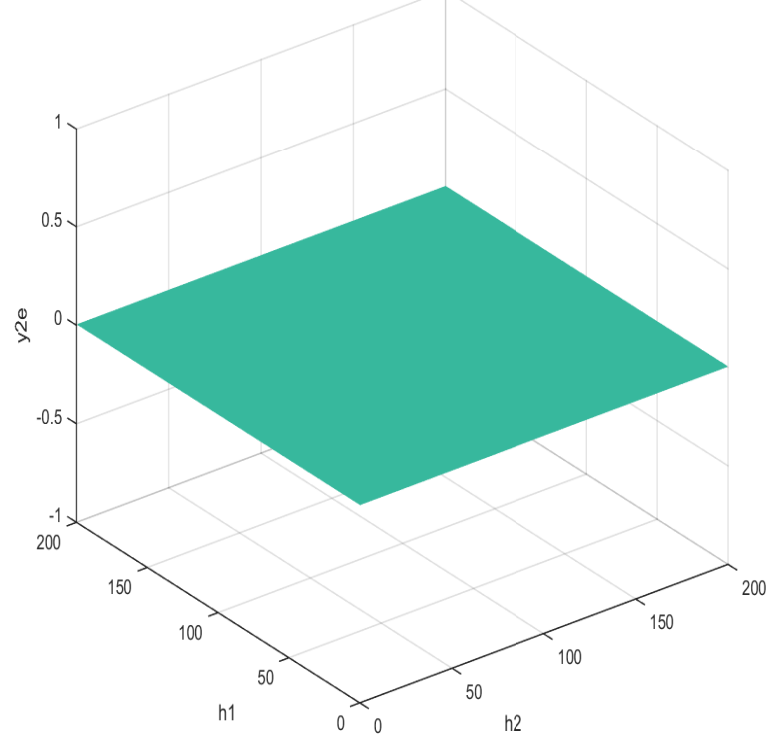

$\mathrm{y}_{2}$ para $\lambda_{2}$ fijo

Figura 5.16: Evolución de $\mathrm{u}_{1} \mathrm{y} \mathrm{u}_{2}$ variando $\lambda_{1} \mathrm{y} \lambda_{2}$ para benchmark 1 
- $\lambda_{1}=\lambda_{2}=60$

- $\mathrm{M}_{1}=\mathrm{M}_{2}=3$

- $\mathrm{n}_{\mathrm{g} 1}=\max \left(\mathrm{n}_{\mathrm{g} 11}, \mathrm{n}_{\mathrm{g} 12}\right)=145$

- $\mathrm{n}_{\mathrm{g} 2}=\max \left(\mathrm{n}_{\mathrm{g} 21}, \mathrm{n}_{\mathrm{g} 22}\right)=145$

Teniendo en cuenta los valores de la Tabla 5.1, la Figura 5.16 y la matriz RGA se obtiene el resultado mostrado en la Figura 5.17.

\subsubsection{Benchmark 2}

Motor-alternador ([55]). Este benchmark es el que se ha usado como ejemplo en el resto del capítulo.

$$
\mathbf{G}=\left(\begin{array}{ll}
\frac{6.1}{(\mathrm{~s}+7)} & \frac{4.6}{(\mathrm{~s}+14.3)} \\
\frac{2.8}{(\mathrm{~s}+6.8)} & \frac{-0.24}{(\mathrm{~s}+4)}
\end{array}\right)
$$

\begin{tabular}{|c|c|c|c|c|c|c|c|}
\hline $\begin{array}{c}\text { Función } \\
\text { transferencia }\end{array}$ & $\begin{array}{c}\text { Constante } \\
\text { de tiempo }\end{array}$ & $\begin{array}{c}\text { Tiempo de } \\
\text { establecimiento }\end{array}$ & Retraso & $\begin{array}{c}\text { Tiempo de } \\
\text { muestreo }\end{array}$ & Pr & M & $\mathrm{n}_{\mathrm{g}}$ \\
\hline $\mathrm{G}_{11}$ & 0,15 & 0,8 & 0 & 0,007 & 22 & 2 & 135 \\
\hline $\mathrm{G}_{12}$ & 0,07 & 0,4 & 0 & 0,007 & 10 & 2 & 67 \\
\hline $\mathrm{G}_{21}$ & 0,1471 & 0,8 & 0 & 0,007 & 22 & 2 & 135 \\
\hline $\mathrm{G}_{22}$ & 0,255 & 1,8 & 0 & 0,007 & 36 & 2 & 293 \\
\hline
\end{tabular}

Cuadro 5.2: Resultados del ajuste para benchmark 2

Las Figuras 5.9 y 5.10 muestran que el valor límite de $\lambda$ está en torno a 0,4. La matriz RGA indica (como se ha visto en la sección 5.4) que los dos factores de peso deben tener un valor similar.

- $\operatorname{Pr}_{1}=\max \left(\operatorname{Pr}_{11}, \operatorname{Pr}_{21}\right)=22$

- $\operatorname{Pr}_{2}=\max \left(\operatorname{Pr}_{21}, \operatorname{Pr}_{22}\right)=36$

- $\lambda_{1}=\lambda_{2}=4$

- $\mathrm{M}_{1}=\mathrm{M}_{2}=2$

- $\mathrm{n}_{\mathrm{g} 1}=\max \left(\mathrm{n}_{\mathrm{g} 11}, \mathrm{n}_{\mathrm{g} 12}\right)=293$

- $\mathrm{n}_{\mathrm{g} 2}=\max \left(\mathrm{n}_{\mathrm{g} 21}, \mathrm{n}_{\mathrm{g} 22}\right)=293$

La figura 5.18 muestra los resultados del ajuste. 


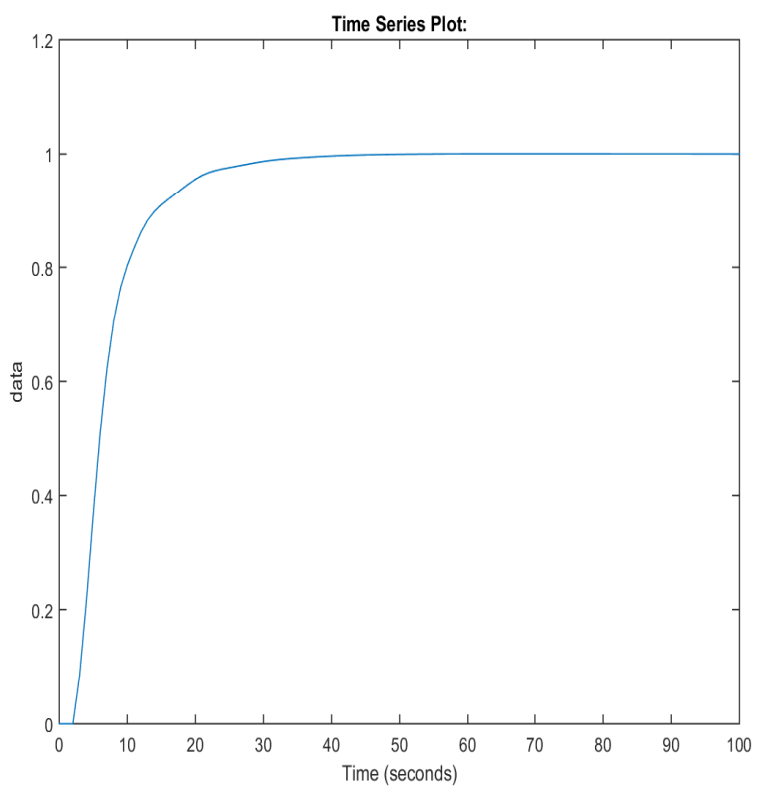

$\mathrm{y}_{1}$

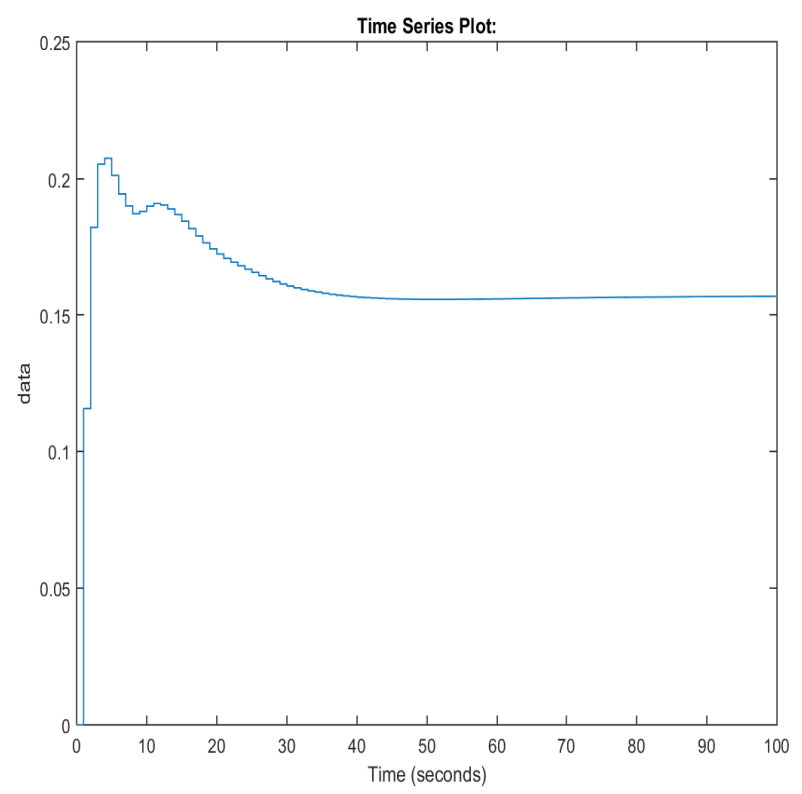

$\mathrm{u}_{1}$

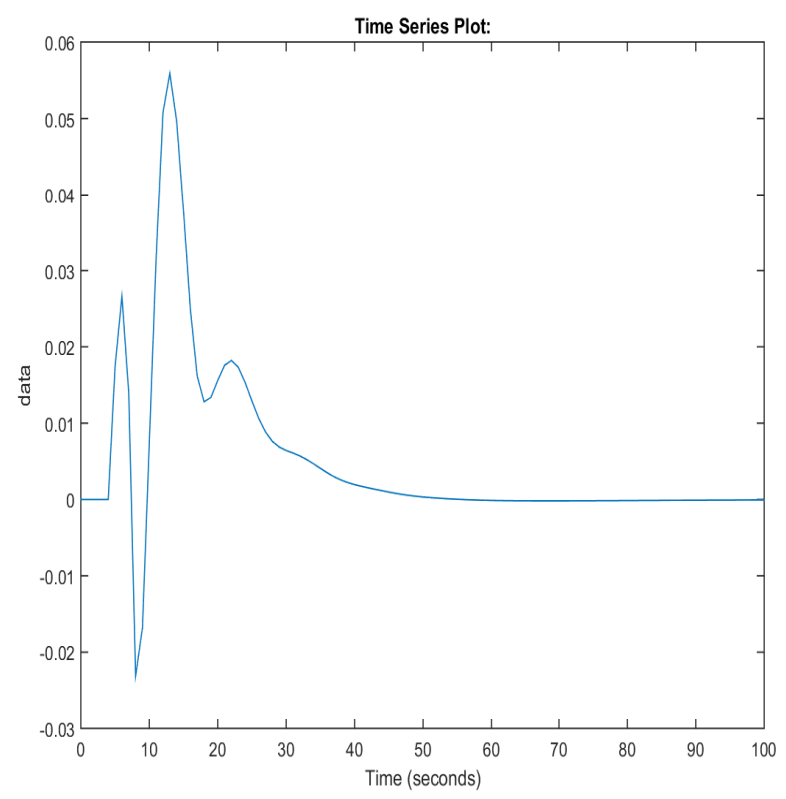

$\mathrm{y}_{2}$

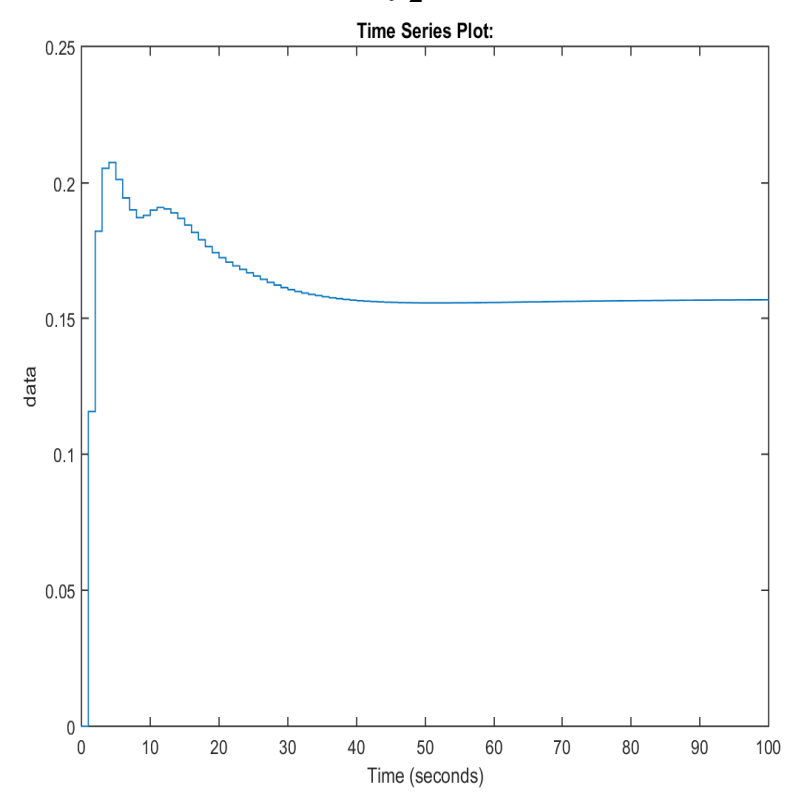

$\mathrm{u}_{2}$

Figura 5.17: Resultado de sintonía para benchmark 1 

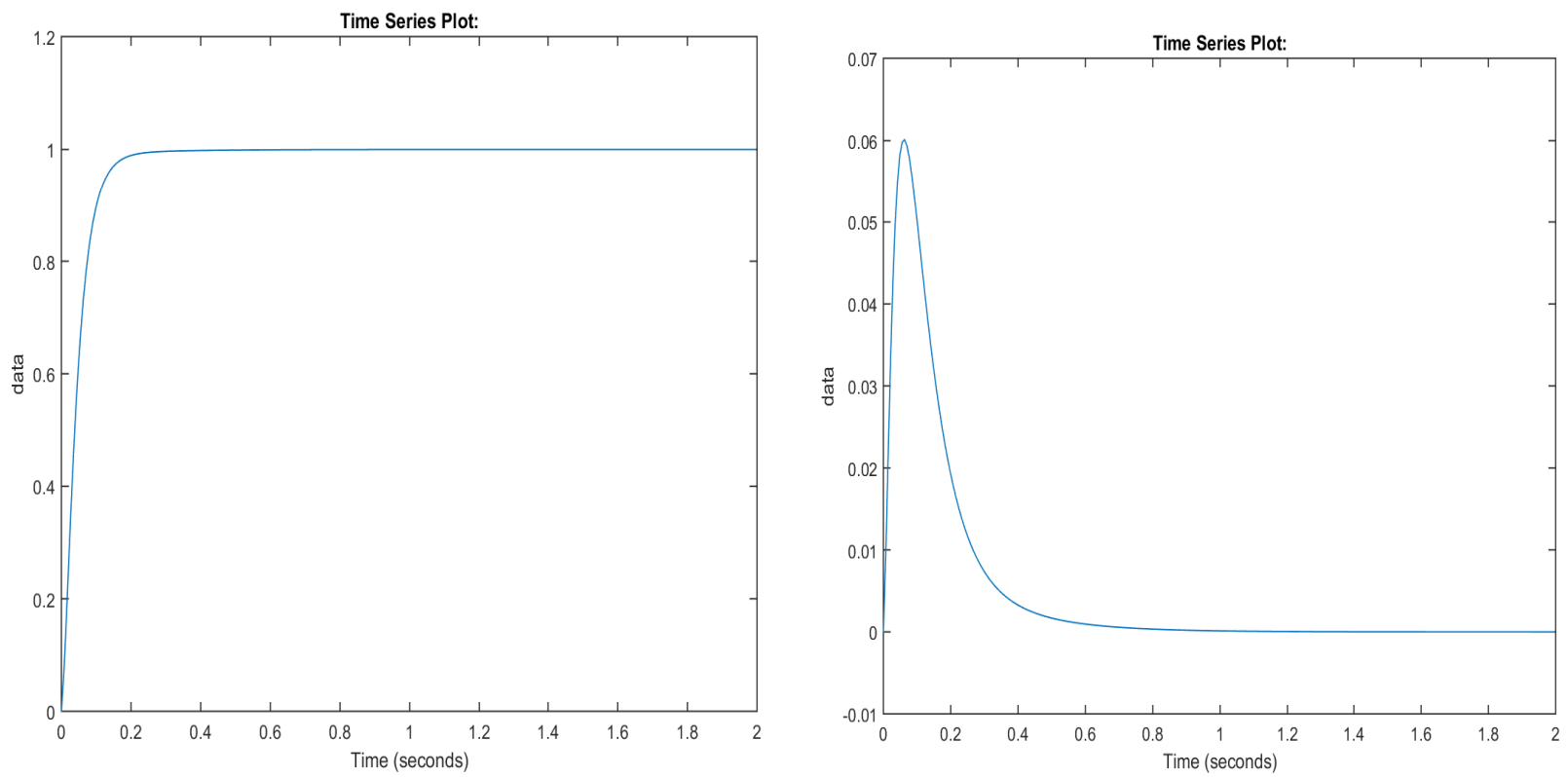

$\mathrm{y}_{1}$

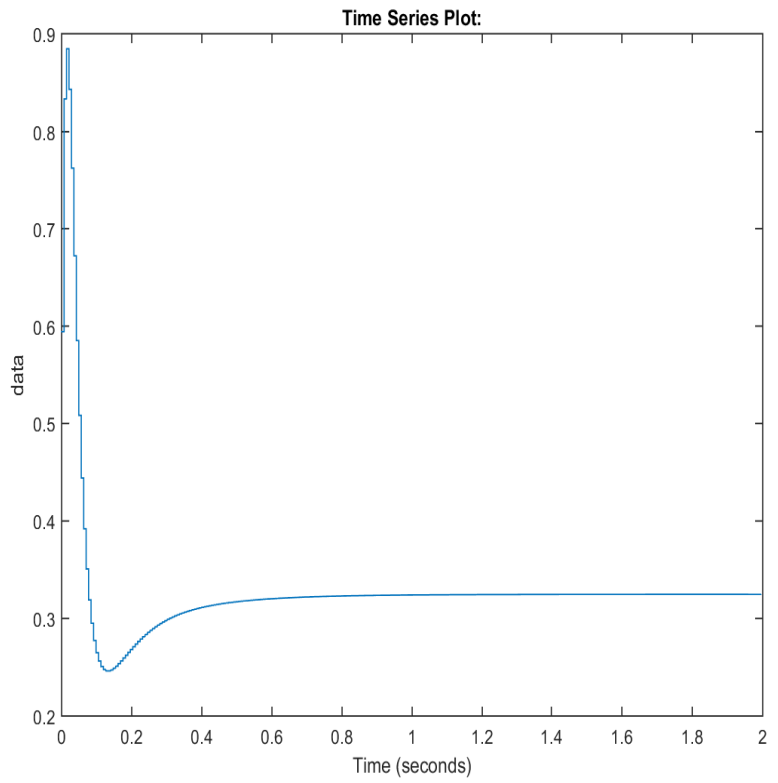

$\mathrm{u}_{1}$

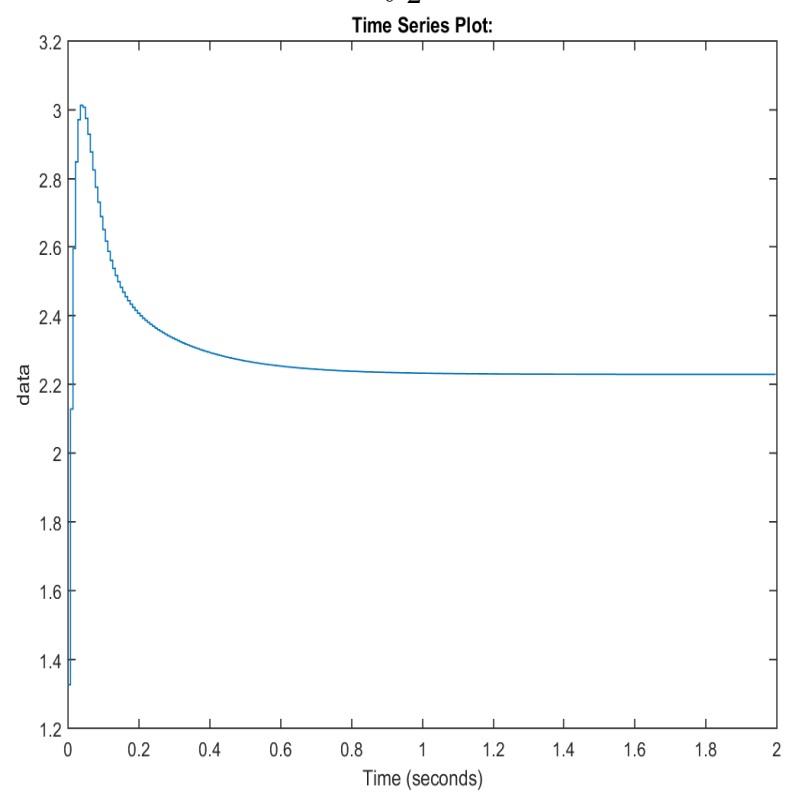

$\mathrm{u}_{2}$

Figura 5.18: Resultado de sintonía para benchmark 2 


\subsubsection{Benchmark 3}

Columna de destilación binaria, [56].

$$
\mathbf{G}=\left(\begin{array}{cc}
\frac{0.878}{(75 \mathrm{~s}+1)} & \frac{-0.864}{(75 \mathrm{~s}+1)} \\
\frac{1.082}{(75 \mathrm{~s}+1)} & \frac{-1.096}{(75 \mathrm{~s}+1)}
\end{array}\right)
$$

\begin{tabular}{|c|c|c|c|c|c|c|c|}
\hline $\begin{array}{c}\text { Función } \\
\text { transferencia }\end{array}$ & $\begin{array}{c}\text { Constante } \\
\text { de tiempo }\end{array}$ & $\begin{array}{c}\text { Tiempo de } \\
\text { establecimiento }\end{array}$ & Retraso & $\begin{array}{c}\text { Tiempo de } \\
\text { muestreo }\end{array}$ & Pr & M & $\mathrm{n}_{\mathrm{g}}$ \\
\hline $\mathrm{G}_{11}$ & 75 & 500 & 0 & 7,5 & 10 & 3 & 70 \\
\hline $\mathrm{G}_{12}$ & 75 & 500 & 0 & 7,5 & 10 & 3 & 70 \\
\hline $\mathrm{G}_{21}$ & 75 & 500 & 0 & 7,5 & 10 & 3 & 70 \\
\hline $\mathrm{G}_{22}$ & 75 & 500 & 0 & 7,5 & 10 & 3 & 70 \\
\hline
\end{tabular}

Cuadro 5.3: Resultados del ajuste para cada benchmark 3

La tabla 5.3 muestra los parámetros obtenidos aplicando las reglas de diseño enunciadas en la sección anterior, excepto el factor de peso. Para el factor de peso será necesario calcular las curvas del primer incremento de control, salida y matriz RGA.

$$
\mathbf{R G A}=\left(\begin{array}{cc}
35 & -34 \\
-34 & 35
\end{array}\right)
$$

Atendiendo a la Figura 5.19 el valor útil límite para $\lambda$ es 0,015 para ambas variables. Teniendo en cuenta la matriz RGA para este sistema, no es aconsejable que los factores de peso tomen valores diferentes. Teniendo en cuenta los valores de la Tabla 5.3, la Figura 5.19 y la matriz RGA se obtiene el resultado mostrado en la Figura 5.20.

- $\operatorname{Pr}_{1}=\max \left(\operatorname{Pr}_{11}, \operatorname{Pr}_{21}\right)=10$

- $\operatorname{Pr}_{2}=\max \left(\operatorname{Pr}_{21}, \operatorname{Pr}_{22}\right)=10$

- $\lambda_{1}=\lambda_{2}=0.015$

- $\mathrm{M}_{1}=\mathrm{M}_{2}=3$

- $\mathrm{n}_{\mathrm{g} 1}=\max \left(\mathrm{n}_{\mathrm{g} 11}, \mathrm{n}_{\mathrm{g} 12}=70\right.$

- $\mathrm{n}_{\mathrm{g} 2}=\max \left(\mathrm{n}_{\mathrm{g} 21}, \mathrm{n}_{\mathrm{g} 22}=70\right.$

\subsection{Comparación con el método de Shridhar Coopper}

Al igual que en capítulo 3 ,se va a comparar el método propuesto con el método de Shridhar Cooper [17]. Se trata de uno de los pocos métodos que han abordado la sintonía del DMC en el caso multivariable. Dicho método (muy similar a su versión para sistemas SISO [16]), consiste en los siguientes pasos: 


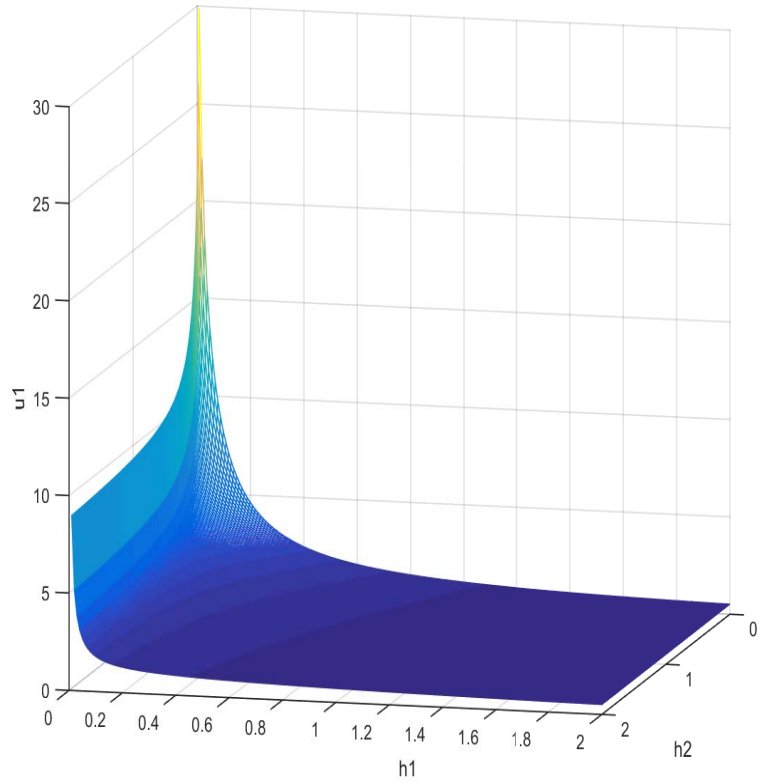

$\mathrm{u}_{1}$ para $\lambda_{1}$ fijo

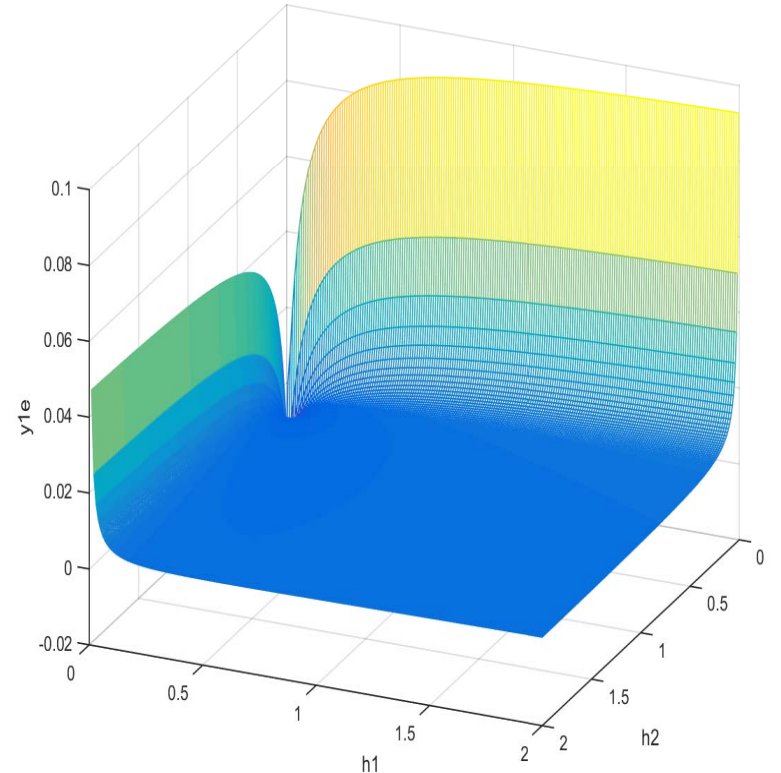

$\mathrm{u}_{2}$ para $\lambda_{1}$ fijo

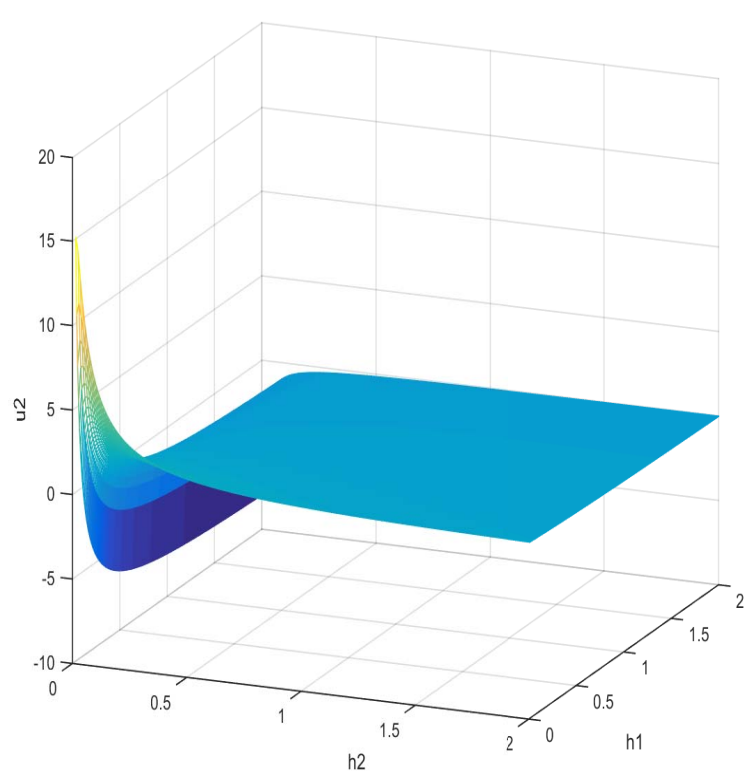

$u_{1}$ para $\lambda_{2}$ fijo

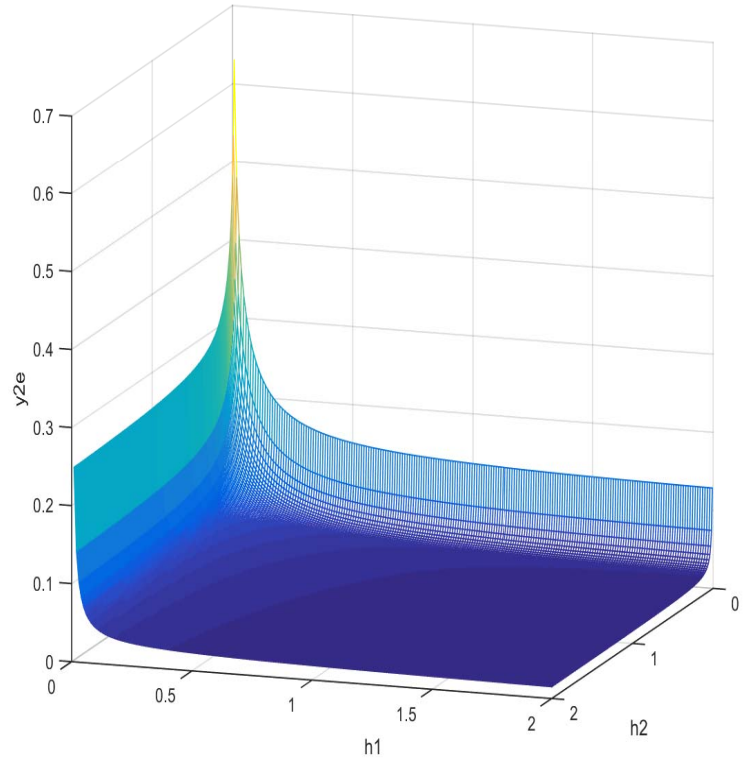

$u_{2}$ para $\lambda_{2}$ fijo

Figura 5.19: Evolución de $\mathrm{u}_{1} \mathrm{y} \mathrm{u}_{2}$ variando $\lambda_{1}$ y $\lambda_{2}$ para benchmark 3 

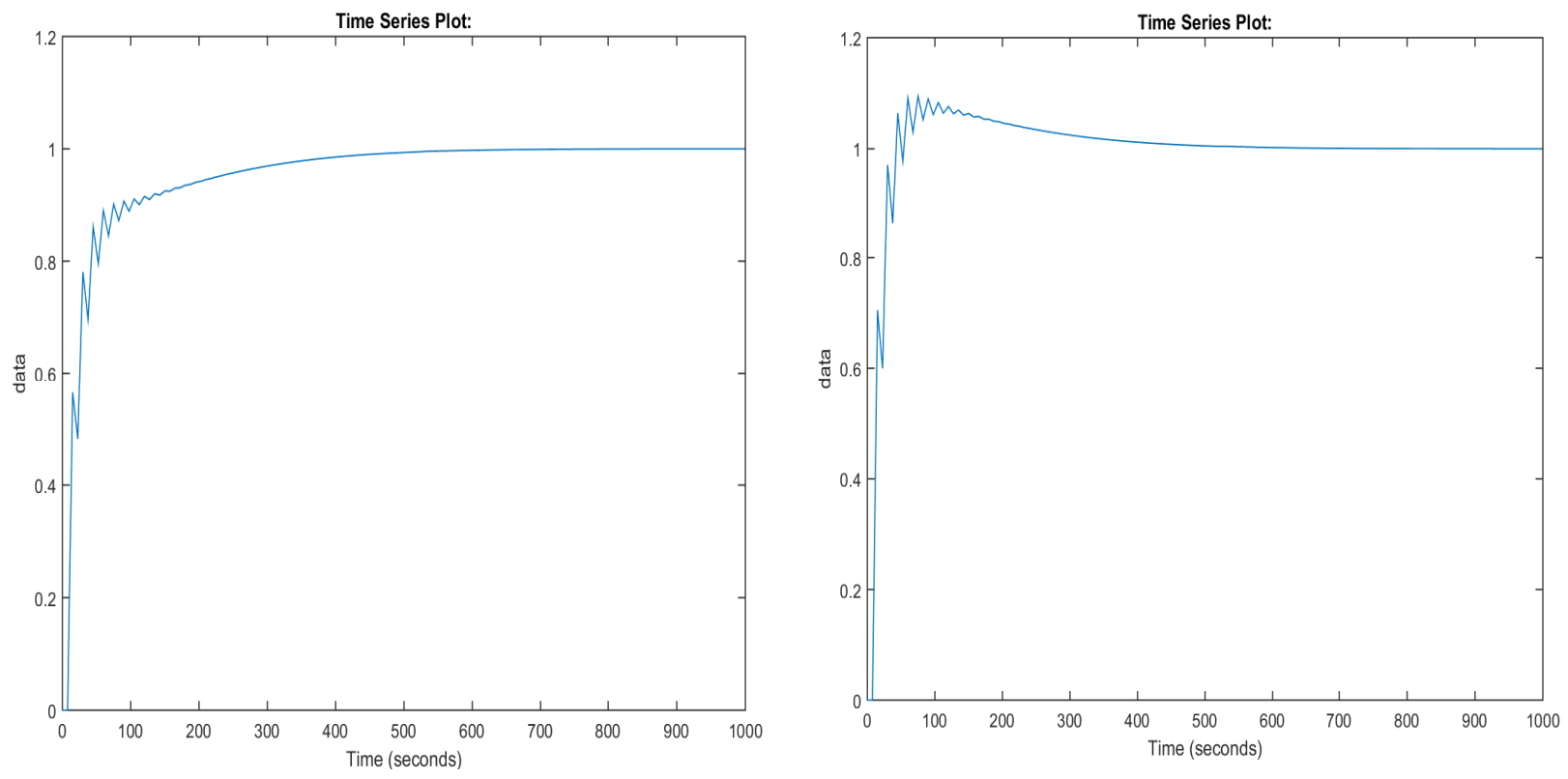

$\mathrm{y}_{1}$

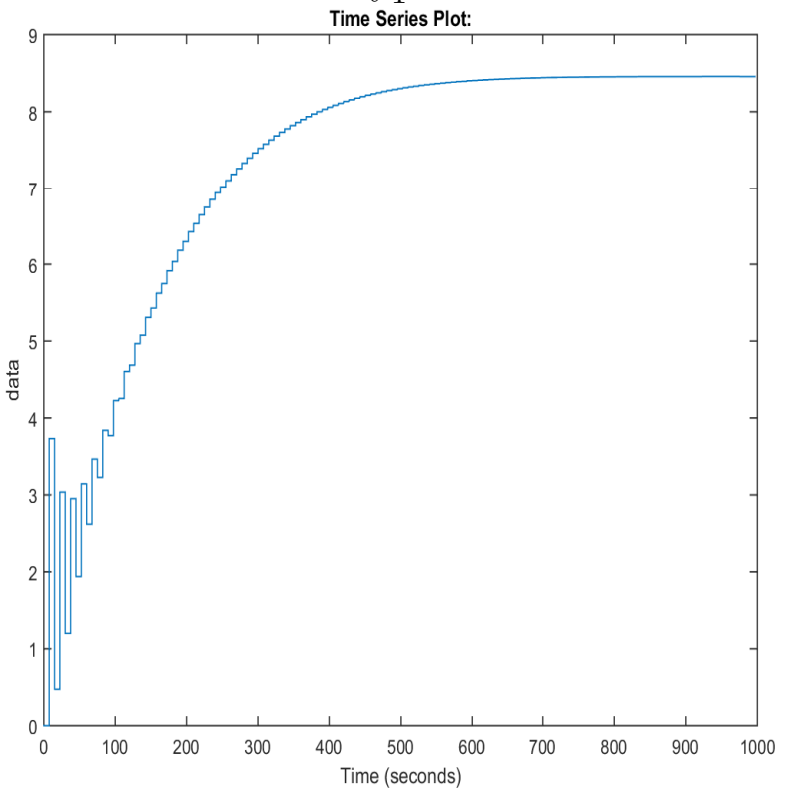

$\mathrm{u}_{1}$

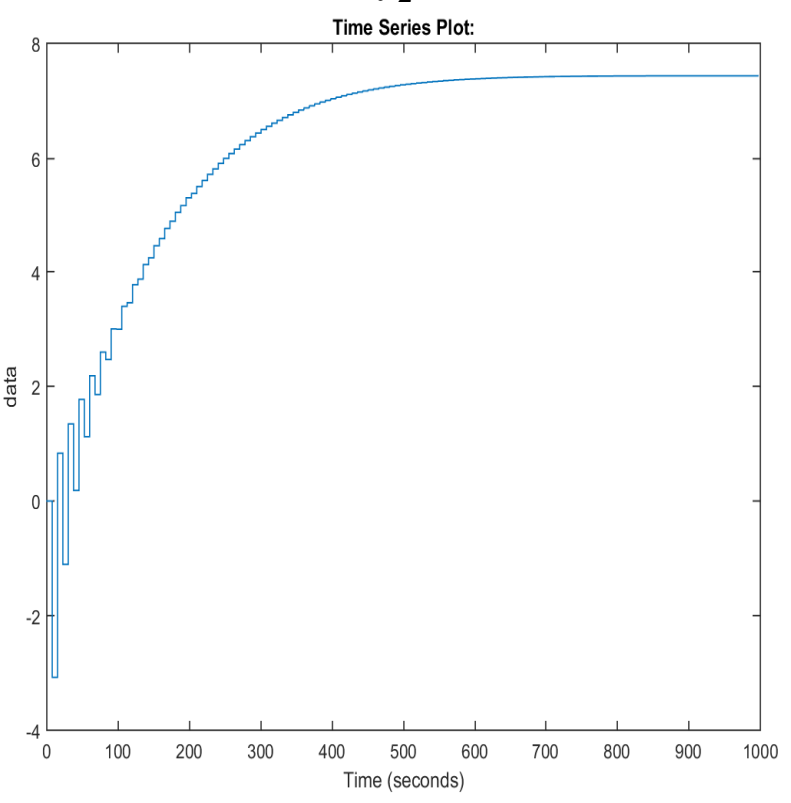

$u_{2}$

Figura 5.20: Resultado de sintonía para benchmark 3 
1. Aproximar el sistema por un FOPDT.

$$
\frac{\mathrm{y}(\mathrm{s})}{\mathrm{u}(\mathrm{s})}=\frac{\mathrm{K}_{\mathrm{p}} \mathrm{e}^{\Theta_{\mathrm{p} s}}}{\tau_{\mathrm{p}} \mathrm{s}+1}
$$

2. Seleccionar, si es posible, un tiempo de muestreo, $\mathrm{T}$, que cumpla $\mathrm{T}=\operatorname{Min}\left(\operatorname{Max}\left(0,1 \tau_{\mathrm{ij}}, 0,5 \Theta_{\mathrm{ij}}\right)\right.$.

3. Calcular el retraso discreto para cada elemento de la matriz de transferencia.

$$
\mathrm{k}_{\mathrm{ij}}=\frac{\Theta_{\mathrm{ij}}}{\mathrm{T}}+1
$$

4. Calcular los horizontes de predicción y de modelo.

$$
\operatorname{Pr}=\mathrm{n}_{\mathrm{g}}=\operatorname{Max}\left(5 \frac{\tau_{\mathrm{ij}}}{\mathrm{T}}+\mathrm{k}_{\mathrm{ij}}\right)
$$

5. Seleccionar el horizonte de control como un número entero entre 1 y 6 .

6. Seleccionar el horizonte de control y el factor de peso.

$$
\lambda_{\mathrm{j}}^{2}=\frac{\mathrm{M}}{500} \sum_{\mathrm{i}=1}^{\mathrm{R}}\left(\gamma_{\mathrm{i}}^{2} \mathrm{~K}_{\mathrm{ij}}^{2}\left(\mathrm{P}-\mathrm{k}_{\mathrm{ij}}-\frac{3}{2} \frac{\tau_{\mathrm{ij}}}{\mathrm{T}}+2-\frac{\mathrm{M}-1}{2}\right)\right)(\mathrm{i}=1,2 \ldots, \mathrm{S})
$$

Debe tenerse en cuenta que este método considera un parámetro más en el DMC de los considerados en este trabajo. En MPC multivariable es habitual el uso de factores de peso adicionales $\left(\mathrm{W}_{\mathrm{y}} \mathrm{y} \mathrm{W}_{\mathrm{u}},[52]\right)$ que ayudan a contrarrestar los efectos de las interacciones entre lazos de control, tal y como puede verse en la ecuación 5.12.

$$
\mathrm{J}=\sum_{\mathrm{j}=1}^{\mathrm{p}} \mathrm{W}_{\mathrm{y}}(\hat{\mathrm{y}}(\mathrm{t}+\mathrm{j} \mid \mathrm{t})-\mathrm{w}(\mathrm{t}+\mathrm{j}))^{2}+\sum_{\mathrm{j}=1}^{\mathrm{m}} \lambda\left(\mathrm{W}_{\mathrm{u}} \Delta \mathrm{u}(\mathrm{t}+\mathrm{j}-1)\right)^{2}
$$

Shridhar y Cooper solo consideran $\mathrm{W}_{\mathrm{y}}$, el factor de peso de la variable controlada, al que designan como $\gamma$.Este factor tiene dos propósitos. El primero de ellos es escalar las medidas de las variables controladas a unidades comparables. El segundo es ajustar el control de una variable controlada en concreto aumentando el peso relativo de su error cuadrático. Estos pesos suelen ser especificados por el usuario y no deberían ser usados como parámetros de diseño. En esta sección se asumirá que son iguales a la unidad. En la tabla 5.4 se pueden ver los resultados del ajuste utilizando el método de Shridhar Cooper. Para el benchmark 1 se han calculado dos ajustes: uno para $\mathrm{T}=1$, para comparar con los resultados de la sección anterior, y otro para $\mathrm{T}=3$, que servirá para comparar el método propuesto con los resultados obtenidos en [17].

La Figura 5.21 muestra la comparación de la respuesta y las señales de control entre el método propuesto y los resultados obtenidos en [17] $(\mathrm{T}=3)$. Los resultados son similares, aunque el método propuesto presenta un tiempo de establecimiento sensiblemente menor. 


\begin{tabular}{|c|c|c|c|c|c|}
\hline Benchmark & $\mathrm{M}$ & Tiempo de muestreo & $\mathrm{P}$ & $\lambda_{1}$ & $\lambda_{2}$ \\
\hline 1 & 2 & 3 & 35 & 24 & 81 \\
\hline 1 & 3 & 1 & 109 & 103 & 350 \\
\hline 2 & 3 & 0,007 & 183 & 0,84 & 0,11 \\
\hline 3 & 3 & 7,5 & 51 & 0,42 & 0,33 \\
\hline
\end{tabular}

Cuadro 5.4: Resultados del ajuste usando el método de Shridhar Cooper

La figura 5.22 muestra la misma comparación, pero para $\mathrm{T}=1$, que es el usado en la sección anterior. La respuesta temporal es similar para ambos tiempos de control.

La figura 5.23 compara el resultado del ajuste obtenido para ambos métodos con el benchmark 2. El método propuesto consigue un tiempo de establecimiento mayor en una de las salidas y un mayor sobrepico en la otra.

La figura 5.24compara el resultado del ajuste obtenido para ambos métodos con el benchmark 3. El método propuesto consigue menor tiempo de establecimiento, pero con ligeras oscilaciones.

El Cuadro 5.5 muestra el error cuadrático medio del método propuesto y del método de Shridhar Cooper respecto a la referencia, un escalón unitario.

\begin{tabular}{|c|c|c|c|}
\hline Benchmark & Salida & Método propuesto & Método Shridhar Cooper \\
\hline $1(\mathrm{~T}=1 \mathrm{~s})$ & $\mathrm{y}_{1}$ & 0,0546 & 0,0638 \\
\hline $1(\mathrm{~T}=1 \mathrm{~s})$ & $\mathrm{y}_{2}$ & $1,58 \mathrm{e}-4$ & 0,0011 \\
\hline $1(\mathrm{~T}=3 \mathrm{~s})$ & $\mathrm{y}_{1}$ & 0,0975 & 0,0963 \\
\hline $1(\mathrm{~T}=3 \mathrm{~s})$ & $\mathrm{y}_{2}$ & $3,26 \mathrm{e}-4$ & 0,0010 \\
\hline 2 & $\mathrm{y}_{1}$ & 0,0158 & 0,0134 \\
\hline 2 & $\mathrm{y}_{2}$ & $1,88 \mathrm{e}-4$ & $1.23 \mathrm{e}-4$ \\
\hline 3 & $\mathrm{y}_{1}$ & 0,0213 & 0,0278 \\
\hline 3 & $2 \mathrm{y}_{2}$ & 0,0177 & 0,0225 \\
\hline
\end{tabular}

Cuadro 5.5: Error cuadrático medio para cada método

De manera general, el método propuesto consigue tiempo de establecimiento similares o inferiores al método de Shridhar Cooper, pero también presenta oscilaciones ligeramente superiores. Todo esto es debido a que el método de Shridhar Cooper utiliza factores de peso más elevados, que necesita para compensar las oscilaciones que se presentan cuando el horizonte de predicción es demasiado alto [52].

\subsection{Columna de destilación de alta pureza}

Este modelo de columna de destilación de alta pureza fue descrito en 1987 ([61] [62]) y desde entonces ha sido empleado por varios autores en sus trabajos (por ejemplo, [56] [63] [64] [65] [66] [67] [68]). Se trata de un sistema MIMO [2x2] altamente no lineal (una característica propia de las columnas de destilación). El comportamiento de este modelo es cercano al de un sistema real. Al no contar con una maqueta multivariable real (a 

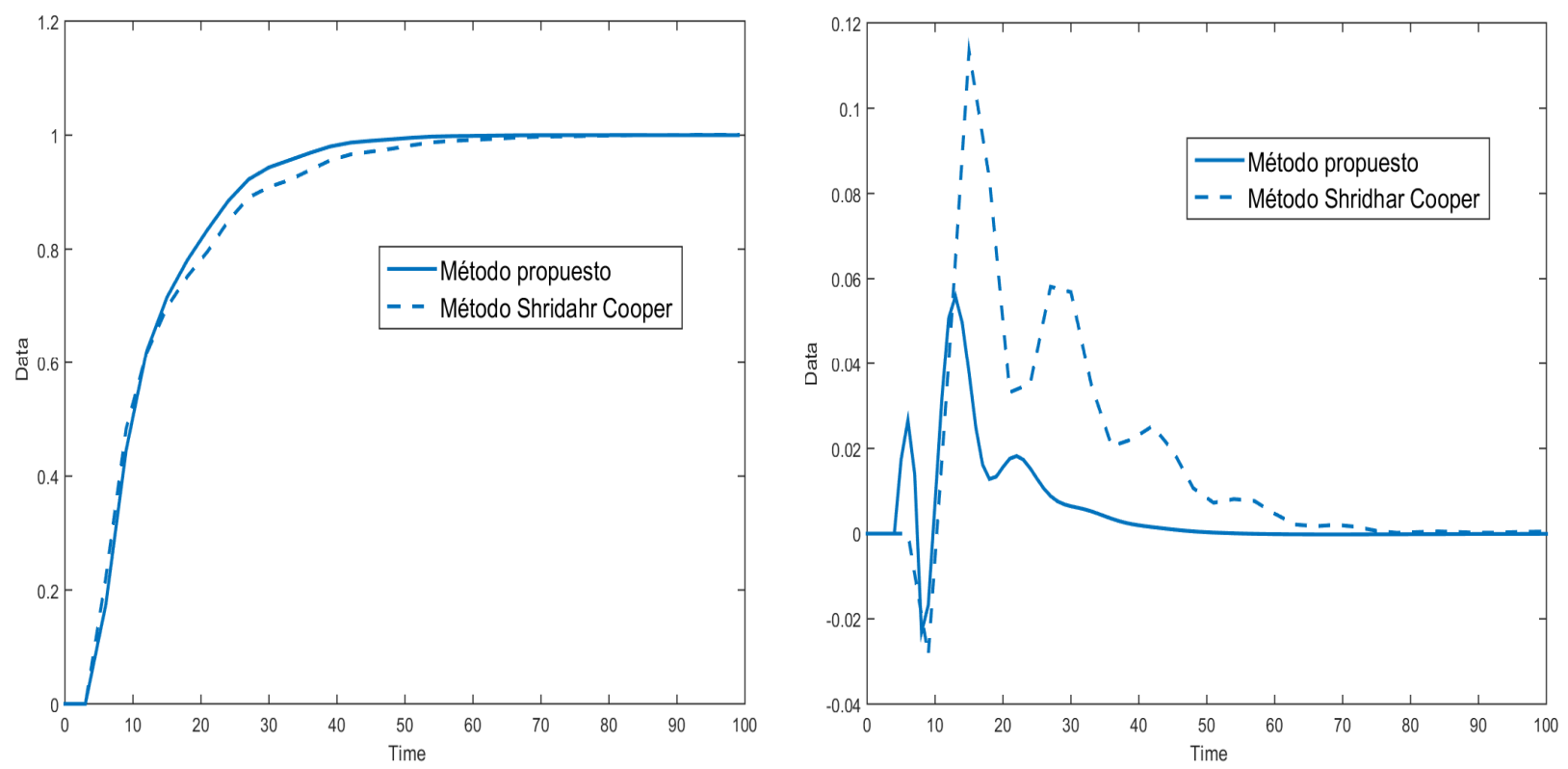

$\mathrm{y}_{1}($ referencia $=1)$

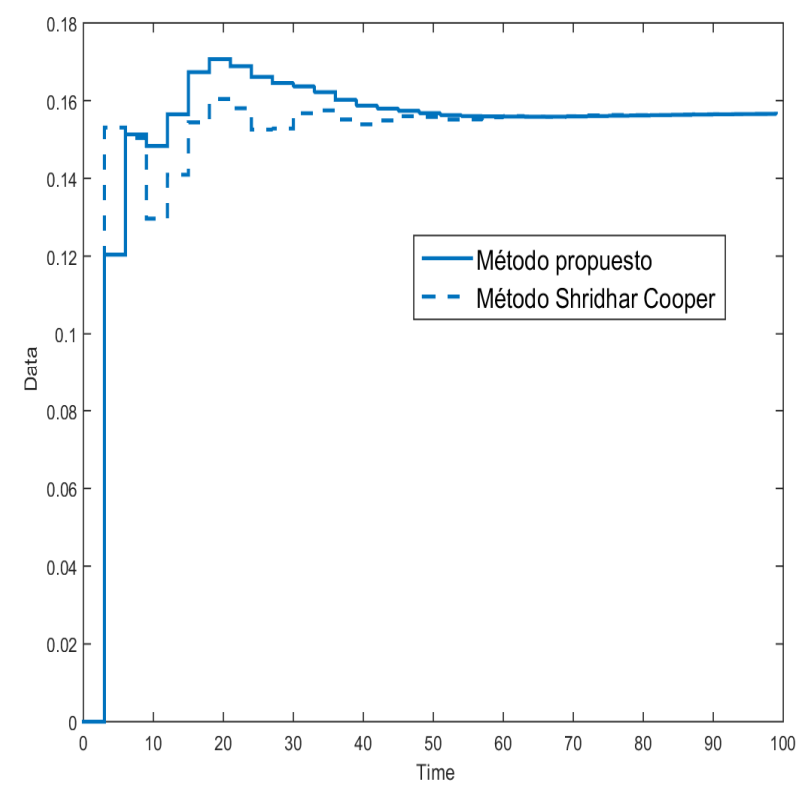

$\mathrm{y}_{2}($ referencia $=0)$

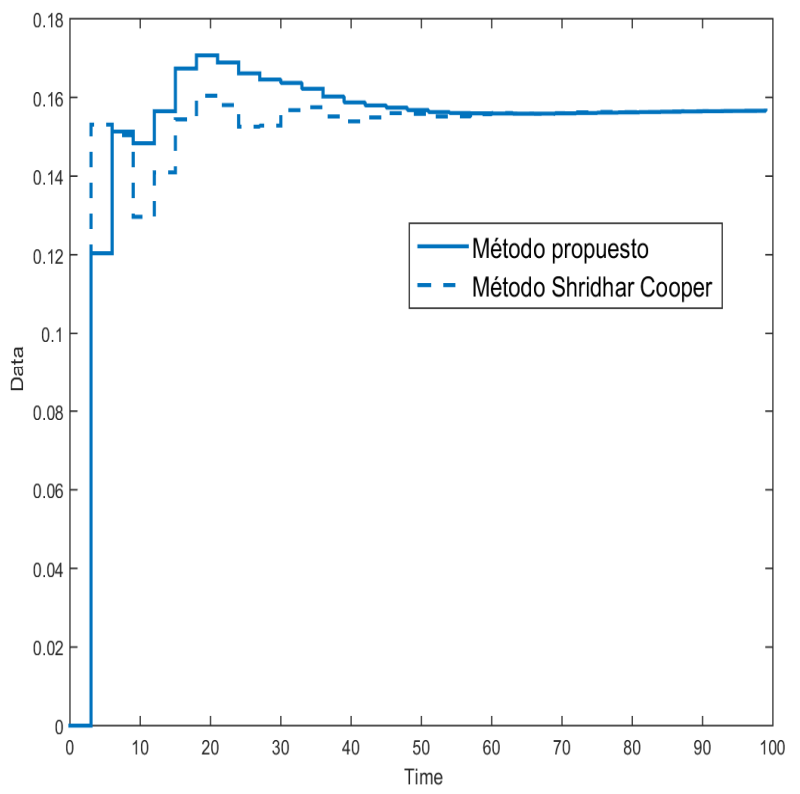

$\mathrm{u}_{1}$

$\mathrm{u}_{2}$

Figura 5.21: Comparativa para benchmark $1(\mathrm{~T}=3)$ 

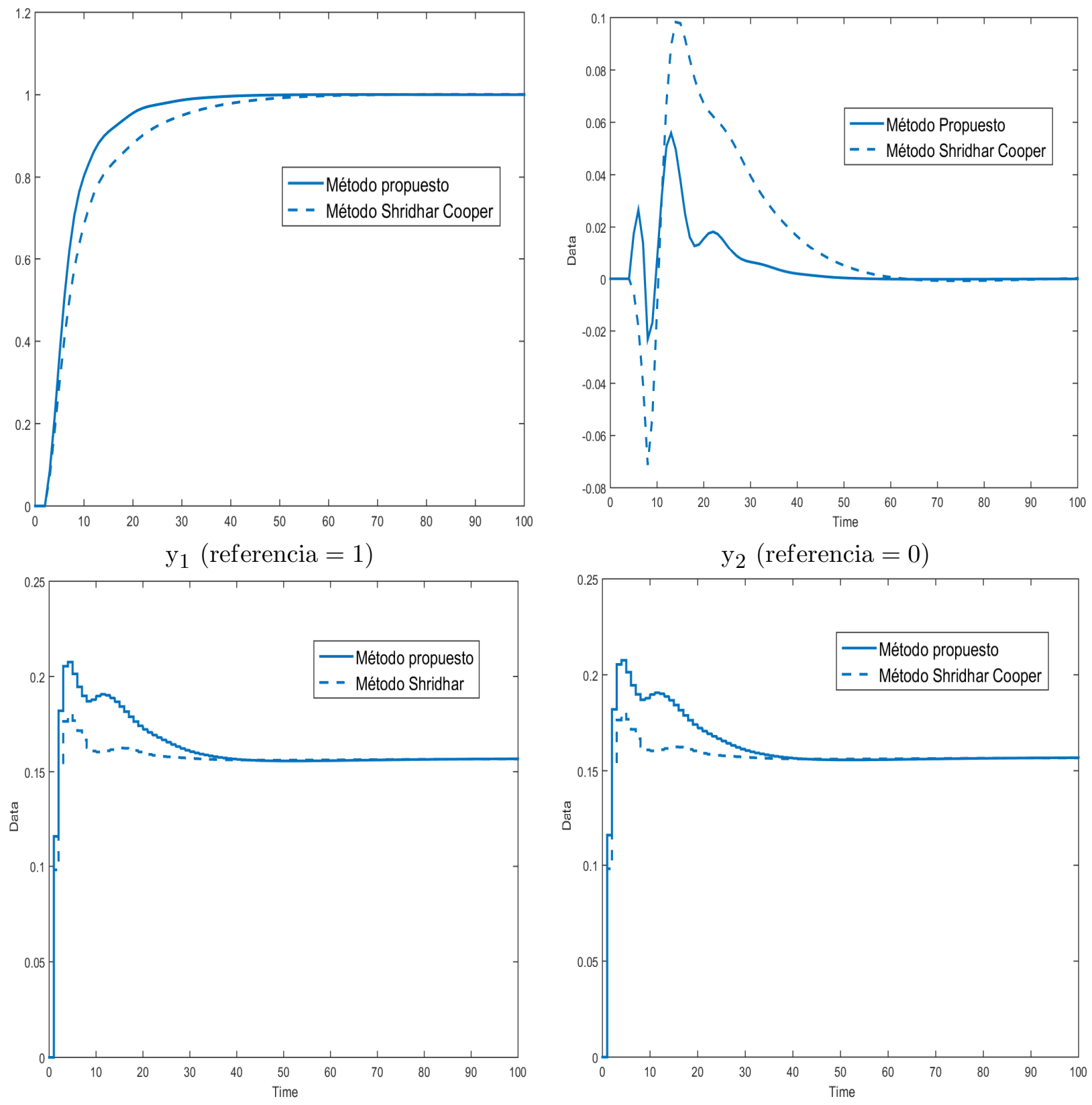

$\mathrm{u}_{1}$

$\mathrm{u}_{2}$

Figura 5.22: Comparativa para benchmark $1(\mathrm{~T}=1)$ 

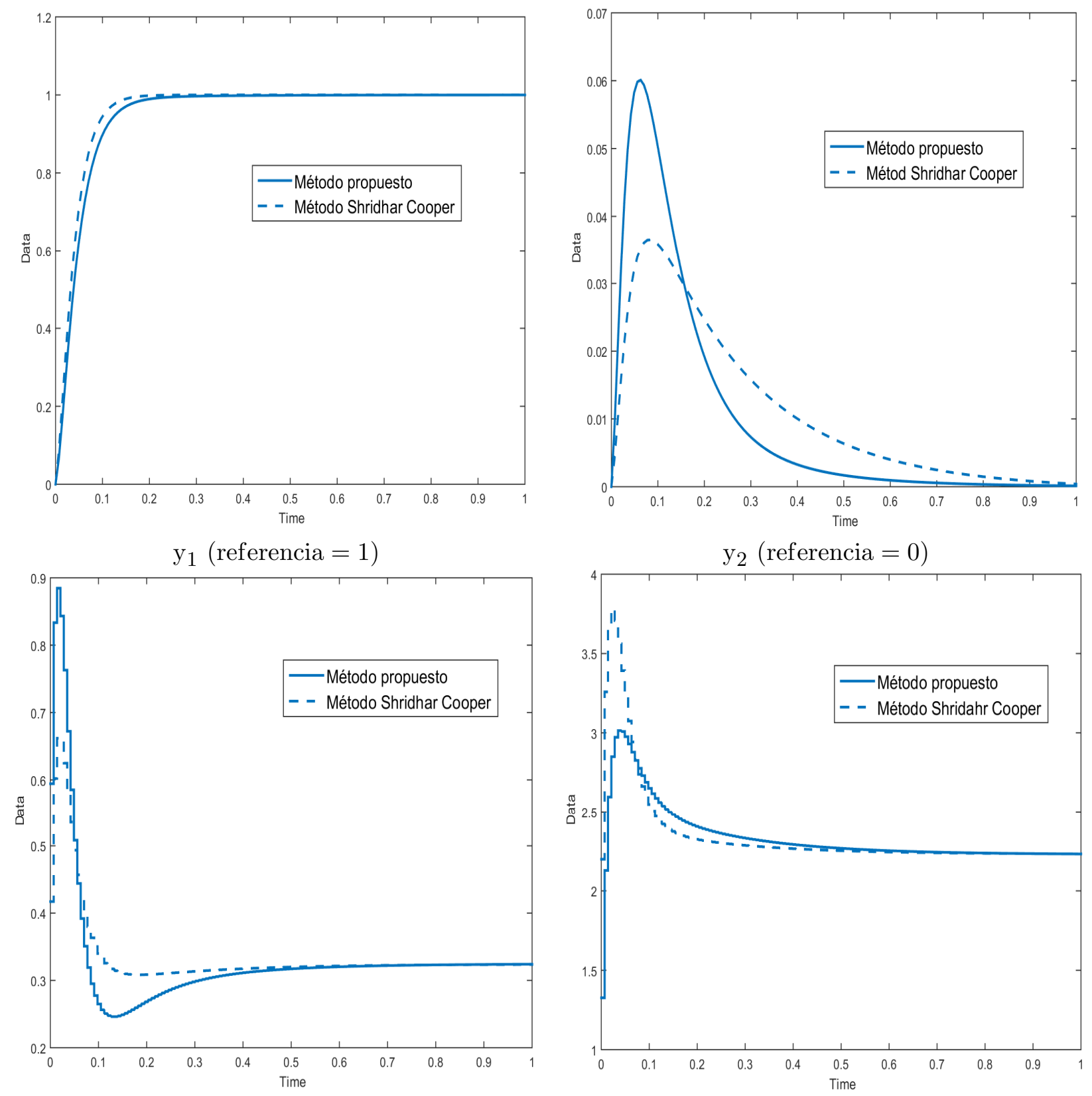

$\mathrm{u}_{1}$

$\mathrm{u}_{2}$

Figura 5.23: Comparativa para benchmark 2 

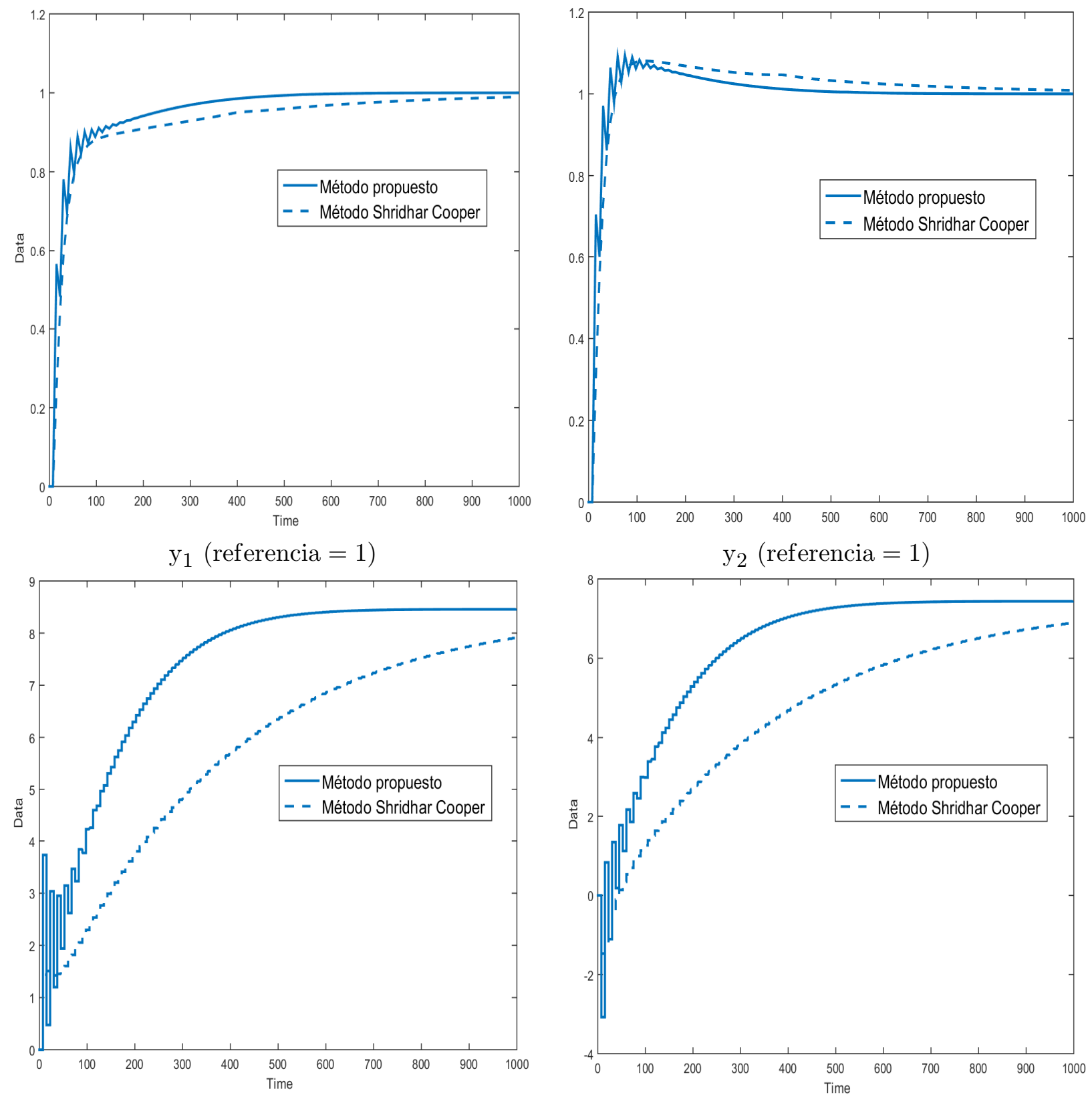

$\mathrm{u}_{1}$

$u_{2}$

Figura 5.24: Comparativa para benchmark 3 
diferencia de lo que pasaba en el capítulo 3) se va a realizar la comprobación final del método con este modelo.

\subsubsection{Modelo de la columna de destilación de alta pureza Nomenclatura e hipótesis}

La Figura muestra el diagrama de la columna. La nomenclatura a utilizar es:

- M: Producto en el fondo de la columna o condensador.

- N: Número de fases de equilibrio teóricas.

- N + 1: Número total de fases incluyendo el condensador.

- $\mathrm{N}_{\mathrm{F}}$ : Fase de alimentación.

- F: Caudal de alimentación

- $z_{\mathrm{F}}$ : Fracción molar de componente volátil en la alimentación.

- $\mathrm{q}_{\mathrm{F}}$ : Fracción de líquido en la alimentación.

- D: Caudal de destilado.

- V: Caudal del evaporador.

- $\mathrm{V}_{\mathrm{T}}$ : Caudal de vapor en la parte superior de la columna.

- L: Caudal de reflujo.

- B: Caudal inferior.

- p: Presión.

- q: Fracción molar líquida de alimentación. 
- x: Fracción molar del componente volátil en el líquido.

- y: Fracción molar del componente volátil en el vapor.

- $\alpha$ : Volatilidad relativa.

- $\kappa$ : Constante VLE (Vapor Liquid Equilibrium) linealizada.

- Subíndices:

- i: Bandeja i (las bandejas se numeran desde el fondo, siendo el evaporador la número 1.

- F: Alimentación.

- D: Destilado.

- B: Fondo.

- T: parte superior.

Se harán las siguientes hipótesis:

- Mezcla binaria

- Presión constante

- Volatilidad relativa constante

- Caudales molares constantes.

- No hay retención de vapor (respuesta de vapor inmediata, $\mathrm{dV}_{\mathrm{T}}=\mathrm{dV}_{\mathrm{B}}$ ).

- Retención $\left(\mathrm{M}_{\mathrm{i}}\right)$ constante de líquido en cada bandeja (respuesta de líquido inmediata, $\left.\mathrm{dL}_{\mathrm{T}}=\mathrm{dL}_{\mathrm{B}}\right)$.

- Equilibrio vapor líquido y mezcla perfecta en todas las fases. 


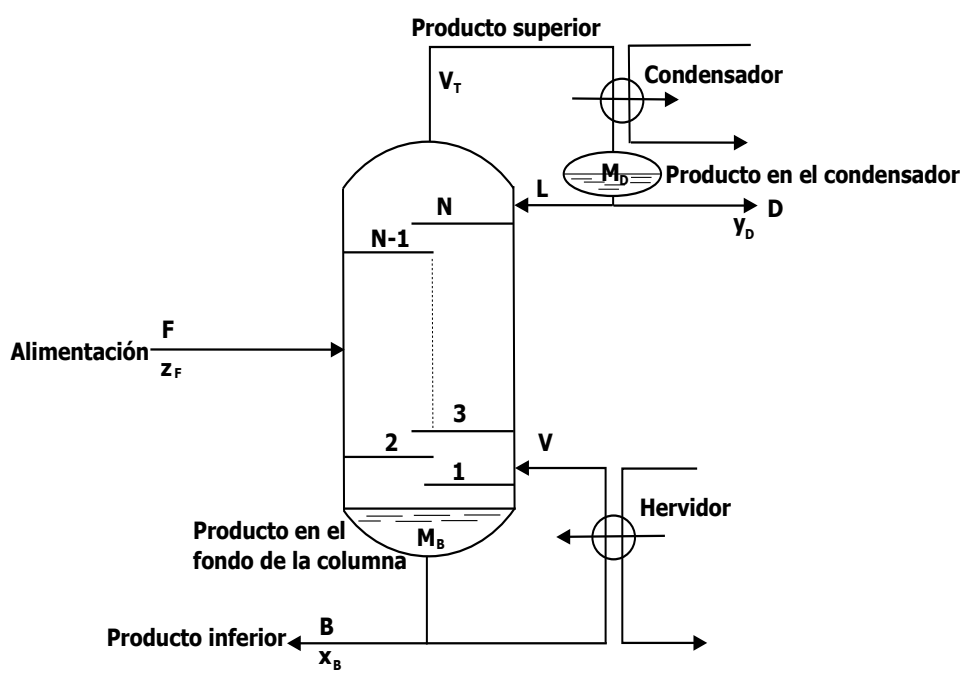

Figura 5.25: Diagrama de la columna de destilación de alta pureza

\section{Modelo no lineal}

El equilibrio de masa para el cambio de retención del componente volátil en cada bandeja $\mathrm{i}=2, \ldots, \mathrm{N}\left(\mathrm{i} \neq \mathrm{N}_{\mathrm{F}}, \mathrm{i} \neq \mathrm{N}_{\mathrm{F}}+1\right)$ :

$$
\mathrm{M}_{\mathrm{i}} \dot{\mathrm{x}}_{\mathrm{i}}=\mathrm{L}_{\mathrm{i}+1} \mathrm{x}_{\mathrm{i}+1}+\mathrm{V}_{\mathrm{i}-1} \mathrm{y}_{\mathrm{i}-1}-\mathrm{L}_{\mathrm{i}} \mathrm{x}_{\mathrm{i}}-\mathrm{V}_{\mathrm{i}} \mathrm{y}_{\mathrm{i}}
$$

Localización por encima de la alimentación $\left(\mathrm{i}=\mathrm{N}_{\mathrm{F}}+1\right)$ :

$$
\mathrm{M}_{\mathrm{i}} \dot{\mathrm{x}}_{\mathrm{i}}=\mathrm{L}_{\mathrm{i}+1} \mathrm{x}_{\mathrm{i}+1}+\mathrm{V}_{\mathrm{i}-1} \mathrm{y}_{\mathrm{i}-1}-\mathrm{L}_{\mathrm{i}} \mathrm{x}_{\mathrm{i}}-\mathrm{V}_{\mathrm{i}} \mathrm{y}_{\mathrm{i}}+\mathrm{F}_{\mathrm{V}} \mathrm{y}_{\mathrm{F}}
$$

Localización por debajo de la alimentación $\left(\mathrm{i}=\mathrm{N}_{\mathrm{F}}\right)$ :

$$
\mathrm{M}_{\mathrm{i}} \dot{\mathrm{x}}_{\mathrm{i}}=\mathrm{L}_{\mathrm{i}+1} \mathrm{x}_{\mathrm{i}+1}+\mathrm{V}_{\mathrm{i}-1} \mathrm{y}_{\mathrm{i}-1}-\mathrm{L}_{\mathrm{i}} \mathrm{x}_{\mathrm{i}}-\mathrm{V}_{\mathrm{i}} \mathrm{y}_{\mathrm{i}}+\mathrm{F}_{\mathrm{L}} \mathrm{x}_{\mathrm{F}}
$$

Evaporador $(\mathrm{i}=1)$ :

$$
\mathrm{M}_{\mathrm{B}} \dot{\mathrm{x}}_{\mathrm{i}}=\mathrm{L}_{\mathrm{i}+1} \mathrm{x}_{\mathrm{i}+1}-\mathrm{V}_{\mathrm{i}} \mathrm{y}_{\mathrm{i}}-\mathrm{B}_{\mathrm{i}} \mathrm{x}_{\mathrm{i}}, \mathrm{x}_{\mathrm{B}}=\mathrm{x}_{1}
$$

Total en el condensador $(\mathrm{i}=\mathrm{N}+1)$ :

$$
\mathrm{M}_{\mathrm{B}} \dot{\mathrm{x}}_{\mathrm{i}}=\mathrm{V}_{\mathrm{i}-1} \mathrm{y}_{\mathrm{i}-1}-\mathrm{L}_{\mathrm{i}} \mathrm{x}_{\mathrm{i}}-\mathrm{D}_{\mathrm{i}} \mathrm{x}_{\mathrm{i}}, \mathrm{y}_{\mathrm{D}}=\mathrm{x}_{\mathrm{N}+1}
$$

Equilibrio vapor líquido en cada bandeja $(\mathrm{i}=1, \ldots, \mathrm{N})$ para volatibilidad constante:

$$
\mathrm{y}_{\mathrm{i}}=\frac{\alpha \mathrm{x}_{\mathrm{i}}}{1+(\alpha-1) \mathrm{x}_{\mathrm{i}}}
$$

Los caudales, asumiendo caudales molares constantes, serán:

$$
\mathrm{i}>\mathrm{N}_{\mathrm{F}}: \mathrm{L}_{\mathrm{i}}=\mathrm{L} \| \mathrm{V}_{\mathrm{i}}=\mathrm{V}+\mathrm{F}_{\mathrm{V}}
$$




$$
\begin{gathered}
\mathrm{i}<\mathrm{N}_{\mathrm{F}}: \mathrm{L}_{\mathrm{i}}=\mathrm{L}+\mathrm{F}_{\mathrm{L}} \| \mathrm{V}_{\mathrm{i}}=\mathrm{V}+\mathrm{F}_{\mathrm{V}} \\
\mathrm{F}_{\mathrm{L}}=\mathrm{q}_{\mathrm{F}} \mathrm{F} \| \mathrm{F}_{\mathrm{V}}=\mathrm{F}-\mathrm{FL} \\
\mathrm{D}=\mathrm{V}_{\mathrm{N}}-\mathrm{L}=\mathrm{V}+\mathrm{F}_{\mathrm{V}}-\mathrm{L} \\
\mathrm{B}=\mathrm{L}_{2}-\mathrm{V}_{1}=\mathrm{L}+\mathrm{F}_{\mathrm{L}}-\mathrm{V}
\end{gathered}
$$

Las composiciones $\mathrm{x}_{\mathrm{F}}$ e $\mathrm{y}_{\mathrm{F}}$ en la fase de líquido y vapor se obtienen resolviendo la ecuación de la evaporación parcial:

$$
\begin{gathered}
\mathrm{Fz}_{\mathrm{F}}=\mathrm{F}_{\mathrm{L}_{\mathrm{F}}}+\mathrm{F}_{\mathrm{V}_{\mathrm{F}}} \\
\mathrm{y}_{\mathrm{F}}=\frac{\alpha \mathrm{x}_{\mathrm{F}}}{1+(\alpha-1) \mathrm{x}_{\mathrm{F}}}
\end{gathered}
$$

Los datos de la columna y sus puntos de operación se muestran en los Cuadros 5.6 y 5.7 .

\begin{tabular}{|c|c|}
\hline Volatibilidad relativa & $\alpha=1.5$ \\
\hline Número de bandejas teóricas & $\mathrm{N}=40$ \\
\hline bandeja de alimentación & $\mathrm{N}_{\mathrm{F}}=21$ \\
\hline Composición de la alimentación & $\mathrm{z}_{\mathrm{F}}=0,5$ \\
\hline Constante de tiempo de la bandeja & $\mathrm{M}_{\mathrm{i}} / \mathrm{F}=0,5$ \\
\hline
\end{tabular}

Cuadro 5.6: Datos columna de destilación

\begin{tabular}{|c|c|c|}
\hline Parámetro & Punto de operación 1 & Punto de operación 2 \\
\hline $\mathrm{y}_{\mathrm{D}}$ & 0,99 & 0,90 \\
\hline $\mathrm{x}_{\mathrm{B}}$ & 0,01 & 0.002 \\
\hline$(\mathrm{L} / \mathrm{D})_{\min }$ & 3,900 & 3,000 \\
\hline $\mathrm{L} / \mathrm{D}$ & 5,413 & 4,935 \\
\hline $\mathrm{D} / \mathrm{F}$ & 0,500 & 0,555 \\
\hline $\mathrm{B} / \mathrm{F}$ & 0,500 & 0,445 \\
\hline $\mathrm{V} / \mathrm{F}$ & 3,206 & 3,291 \\
\hline $\mathrm{L} / \mathrm{F}$ & 2,706 & 2.737 \\
\hline
\end{tabular}

Cuadro 5.7: Puntos de funcionamiento columna de destilación

\section{Modelo lineal}

Se linealiza el equilibrio de masa en cada bandeja $\left(\mathrm{dL}_{\mathrm{i}}=\mathrm{dL}, \mathrm{dV} \mathrm{V}_{\mathrm{i}}=\mathrm{dV}\right)$ :

$$
\mathrm{M}_{\mathrm{i}} \dot{\mathrm{x}}_{\mathrm{i}}=\mathrm{L}_{\mathrm{i}+1} \mathrm{dx}_{\mathrm{i}+1}-\left(\mathrm{L}_{\mathrm{i}}+\mathrm{K}_{\mathrm{i}} \mathrm{V}_{\mathrm{i}}\right) \mathrm{dx}_{\mathrm{i}}+\mathrm{K}_{\mathrm{i}-1} \mathrm{~V}_{\mathrm{i}-1} \mathrm{dx}_{\mathrm{i}-1}+\left(\mathrm{x}_{\mathrm{i}+1}-\mathrm{x}_{\mathrm{i}}\right) \mathrm{dL}-\left(\mathrm{y}_{\mathrm{i}+1}-\mathrm{y}_{\mathrm{i}}\right) \mathrm{dV}
$$


$\mathrm{K}_{\mathrm{i}}$ es la constante VLE linealizada:

$$
\mathrm{K}_{\mathrm{i}}=\frac{\mathrm{dy}_{\mathrm{i}}}{\mathrm{dx}_{\mathrm{i}}}=\frac{\alpha}{\left(1+(\alpha-1) \mathrm{x}_{\mathrm{i}}\right)^{2}}
$$

$\mathrm{Y}_{\mathrm{i}}, \mathrm{x}_{\mathrm{i}}, \mathrm{L}_{\mathrm{i}} \mathrm{y} \mathrm{V}_{\mathrm{i}}$ son los valores en estado estacionario en el punto nominal de operación. Este modelo pues escribirse en forma de estado de espacios:

$$
\dot{\mathrm{x}}=\mathrm{Ax}+\mathrm{Buy}=\mathrm{Cx}
$$

Donde $\mathrm{x}=\left(\mathrm{dx}_{1}, \ldots, \mathrm{dx}_{\mathrm{N}+1}\right)^{\mathrm{T}}$ son las composiciones de las bandejas, $\mathrm{u}=(\mathrm{dL}, \mathrm{dV})^{\mathrm{T}}$ son las variables manipuladas e $\mathrm{y}=\left(\mathrm{dy}_{\mathrm{D}}, \mathrm{dx}_{\mathrm{B}}\right)^{\mathrm{T}}$ son las variables controladas. La matriz de estado $\mathrm{A}=\mathrm{a}_{\mathrm{ij}}$ es tri-diagonal:

$$
\begin{gathered}
\mathrm{i} \neq \mathrm{N}+1: \mathrm{a}_{\mathrm{i}, \mathrm{i}+1}=\frac{\mathrm{L}_{\mathrm{i}+1}}{\mathrm{M}_{\mathrm{i}}} \\
\mathrm{a}_{\mathrm{i}, \mathrm{i}}=\frac{-\mathrm{L}_{\mathrm{i}}+\mathrm{K}_{\mathrm{i}} \mathrm{V}_{\mathrm{i}}}{\mathrm{M}_{\mathrm{i}}} \\
\mathrm{i} \neq 1: \mathrm{a}_{\mathrm{i}, \mathrm{i}-1}=\frac{\mathrm{K}_{\mathrm{i}-1} \mathrm{~V}_{\mathrm{i}-1}}{\mathrm{M}_{\mathrm{i}}}
\end{gathered}
$$

Matriz de entrada:

$$
\begin{gathered}
\mathrm{i} \neq \mathrm{N}+1: \mathrm{b}_{\mathrm{i}, 1}=\frac{\left(\mathrm{x}_{\mathrm{i}+1}-\mathrm{x}_{\mathrm{i}}\right)}{\mathrm{M}_{\mathrm{i}}}, \mathrm{b}_{\mathrm{N}+1,1}=0 \\
\mathrm{i} \neq \mathrm{N}+1 \mathrm{i} \neq \mathrm{n}+1: \mathrm{b}_{\mathrm{i}, 2}=\frac{\left(\mathrm{y}_{\mathrm{i}}-\mathrm{y}_{\mathrm{i}-1}\right)}{\mathrm{M}_{\mathrm{i}}}, \mathrm{b}_{\mathrm{N}+1,2}=0, \mathrm{~b}_{1,2}=\frac{\left(\mathrm{y}_{1}-\mathrm{x}_{1}\right)}{\mathrm{M}_{\mathrm{i}}}
\end{gathered}
$$

Matriz de salida, C:

$$
\mathrm{C}=\left(\begin{array}{lllll}
0 & 0 & \ldots & 0 & 1 \\
1 & 0 & \ldots & 0 & 1
\end{array}\right)
$$

Se supone que el tambor del condensador está bajo un control de nivel perfecto.

$$
\mathrm{V}=\mathrm{L}+\mathrm{D}
$$

En caso de utilizar un conjunto diferente de variables manipuladas, $U=(d D, d V)^{\mathrm{T}}$, el nuevo modelo se obtendría por transformación lineal:

$$
\left(\begin{array}{l}
\mathrm{dD} \\
\mathrm{dV}
\end{array}\right)=\left(\begin{array}{cc}
-1 & 1 \\
0 & 1
\end{array}\right)\left(\begin{array}{l}
\mathrm{dL} \\
\mathrm{dV}
\end{array}\right)
$$




\subsubsection{Control mediante DMC}

La columna descrita en los apartados anteriores es un sistema MIMO [2x2] con las siguientes variables:

- Variables controladas

1. Fracción molar del componente volátil en el destilado, $\mathrm{y}_{\mathrm{D}}$.

2. Fracción molar del componente volátil en el producto inferior, $\mathrm{x}_{\mathrm{B}}$.

- Variables manipuladas

1. Caudal de reflujo, L.

2. Caudal del calentador, V.

El primer paso necesario para diseñar el controlador DMC será obtener su modelo de respuesta a escalón. Para obtener los coeficientes de respuesta a escalón se aplicará un escalón de -0,1 a la variable controlada 1 (L, caudal de reflujo) y un escalón de 0,1 a la variable controlada 2 ( $\mathrm{V}$, caudal del calentador). Los resultados se representan en la Figura 5.26

El modelo a emplear para el DMC se construirá a partir de estas respuesta a escalón realizando los siguientes pasos:

- Dividir el resultado entre el valor de escalón utilizado para que los valores correspondan a un escalón unitario.

- Convertir esos valores de salida en relativos respecto al punto de funcionamiento.

Los modelos resultantes de aplicar estos pasos se pueden ver en la Figura 5.27. El cuadro 5.8 muestra los parámetros de los FOPDT equivalentes y los valores iniciales de ajuste.

\begin{tabular}{|c|c|c|c|c|c|c|c|}
\hline $\begin{array}{c}\text { Función } \\
\text { transferencia }\end{array}$ & $\begin{array}{c}\text { Constante } \\
\text { de tiempo }\end{array}$ & $\begin{array}{c}\text { Tiempo de } \\
\text { establecimiento }\end{array}$ & Retraso & $\begin{array}{c}\text { Tiempo de } \\
\text { muestreo }\end{array}$ & Pr & M & $\mathrm{n}_{\mathrm{g}}$ \\
\hline $\mathrm{G}_{11}$ & 109 & 450 & 0 & 2 & 55 & 3 & 250 \\
\hline $\mathrm{G}_{12}$ & 110 & 450 & 20 & 2 & 55 & 3 & 250 \\
\hline $\mathrm{G}_{21}$ & 24 & 150 & 0 & 2 & 22 & 3 & 250 \\
\hline $\mathrm{G}_{22}$ & 23 & 150 & 0 & 2 & 12 & 3 & 250 \\
\hline
\end{tabular}

Cuadro 5.8: Resultados del ajuste para columna de destilación

Para hallar los valores correctos para los factores de peso será necesario calcular las curvas de primer incremento de control (Figura 5.28) y la matriz RGA. Las curvas muestran que el valor útil máximo para los factores de peso es de 0,01 y la matriz RGA (Ecuación 5.30 ) demuestra que ambas variables controladas $\left(\mathrm{y}_{\mathrm{D}}\right.$ e $\mathrm{x}_{\mathrm{B}}$ ) están fuertemente influenciadas por las variables manipuladas $(\mathrm{L} \mathrm{y} \mathrm{V}$ ). Los valores de los componentes de la matriz 

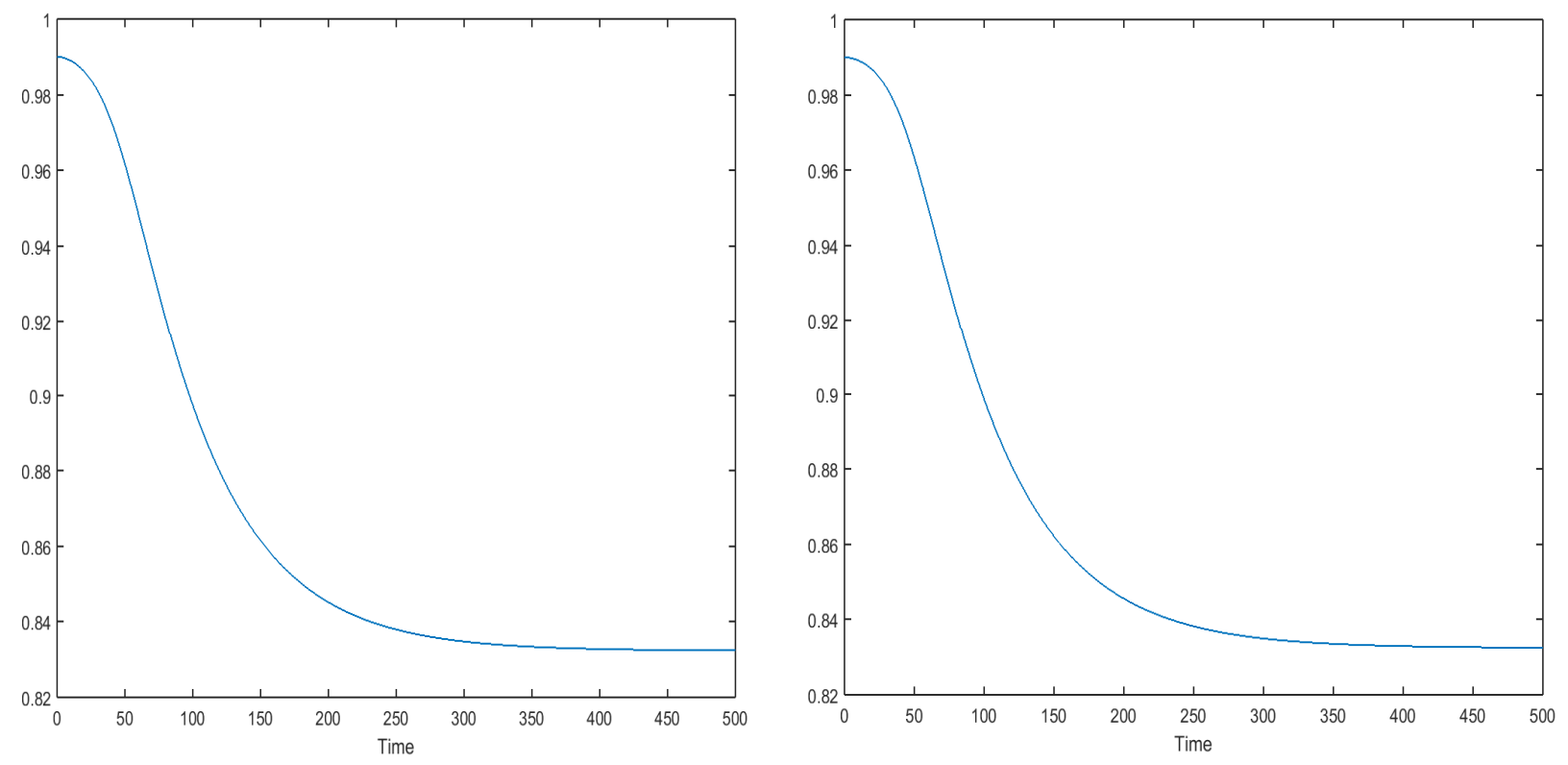

$\mathrm{g}_{11}$

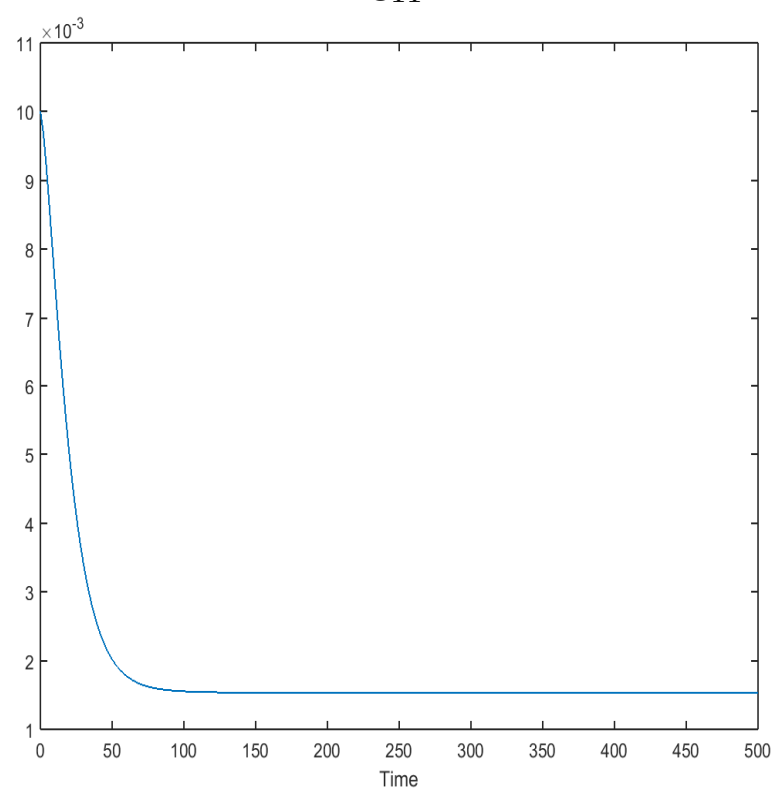

$\mathrm{g}_{12}$

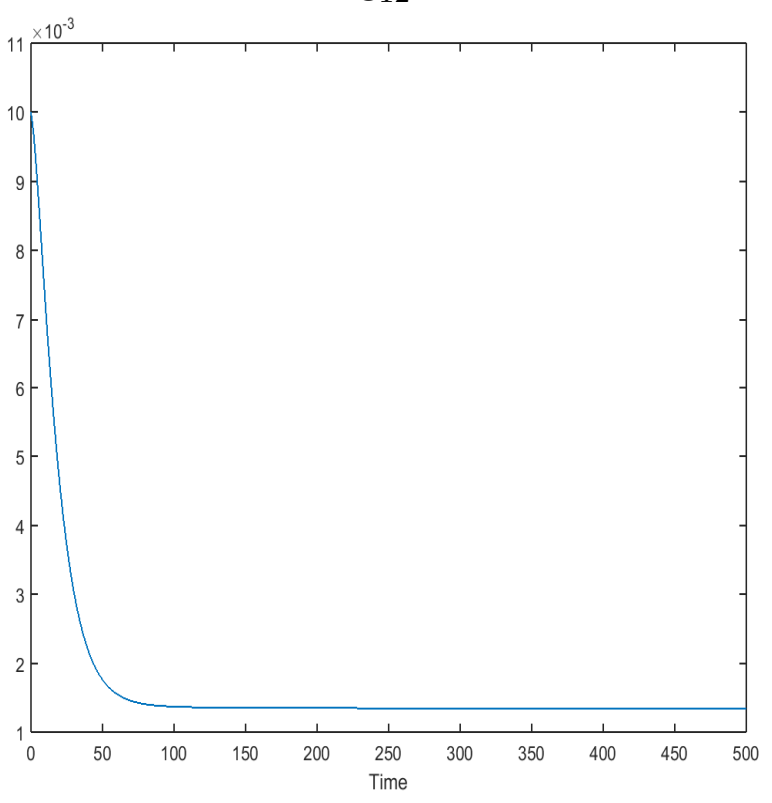

$\mathrm{g}_{21}$

$\mathrm{g}_{22}$

Figura 5.26: Respuesta escalón columna destilación 

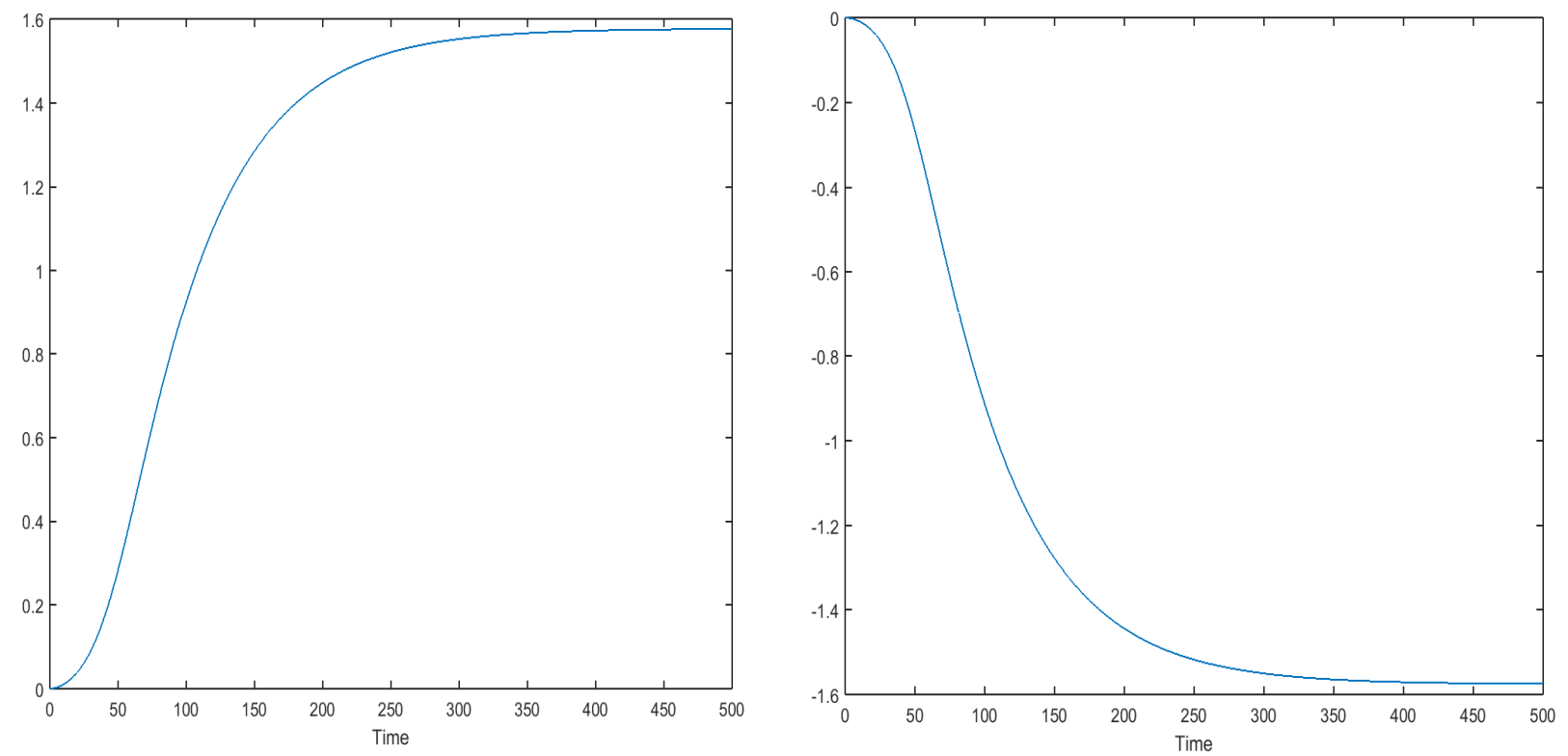

$g_{11}$
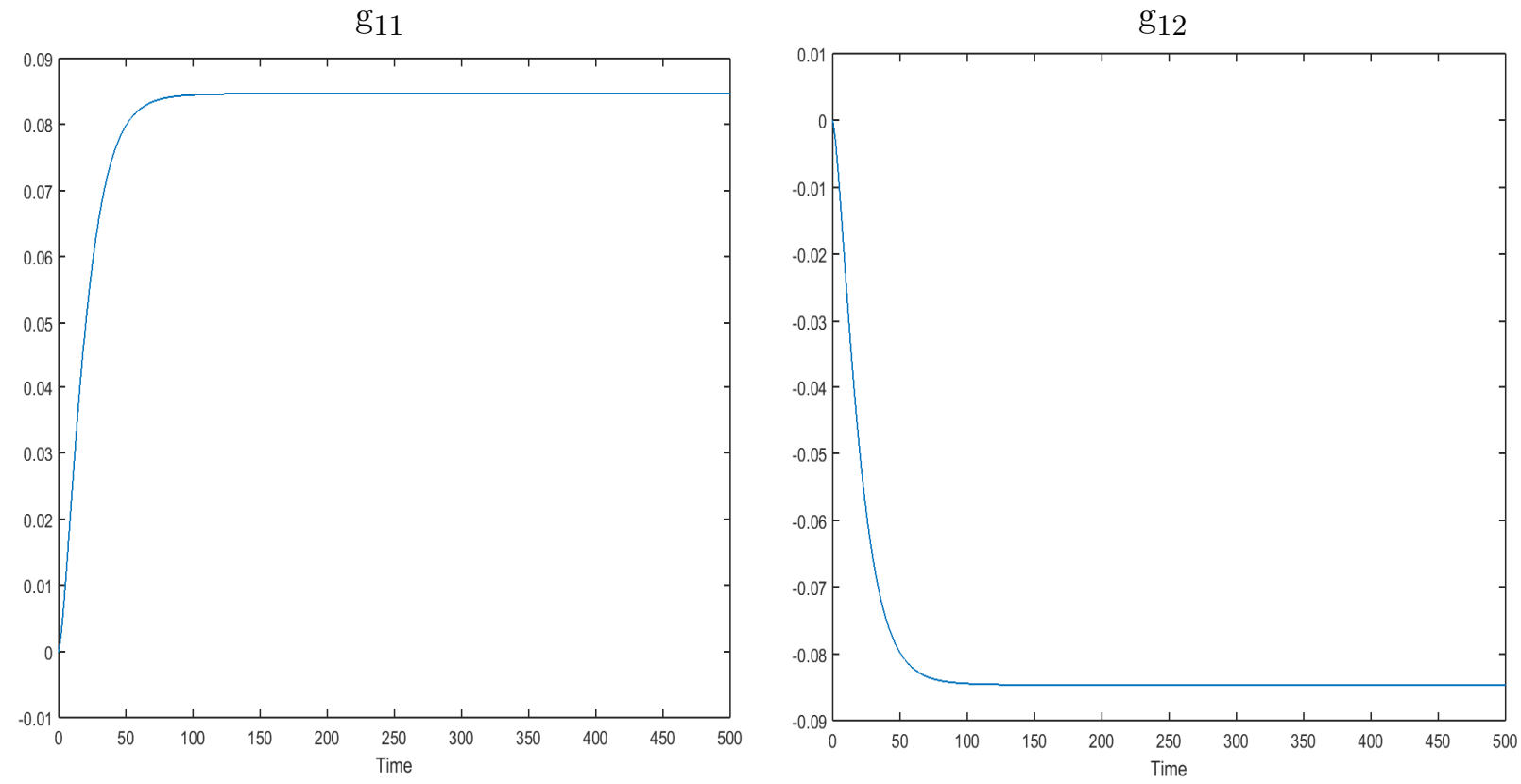

$\mathrm{g}_{21}$

$\mathrm{g}_{22}$

Figura 5.27: Modelo de respuesta escalón columna destilación 
RGA (muy superiores a 1) indican que la ganancia en lazo cerrado es inferior a la ganancia en lazo abierto debido a las interacciones. Dicha reducción tienen signo contrario al de lazo abierto en dos casos. Además la naturaleza simétrica de la matriz nos indica que la magnitud de esta influencia es la misma para ambas variables manipuladas. El estudio de la matriz RGA nos indica que las interacciones son fuertes y que son de la misma magnitud, por lo no se debería penalizar a una variable manipulada más que otra y el factor de peso de ambas variables manipuladas debe ser el mismo.

$$
\mathbf{R G A}=\left(\begin{array}{cc}
29,0068 & -28,0068 \\
-28,0068 & 29,0068
\end{array}\right)
$$

A partir de las gráficas y modelos obtenidos, se pueden deducir los siguientes parámetros:

- $\operatorname{Pr}_{1}=\max \left(\operatorname{Pr}_{11}, \operatorname{Pr}_{21}\right)=55$

- $\operatorname{Pr}_{2}=\max \left(\operatorname{Pr}_{21}, \operatorname{Pr}_{22}\right)=55$

- $\lambda_{1}=\lambda_{2}=0.01$

- $\mathrm{M}_{1}=\mathrm{M}_{2}=3$

- $\mathrm{n}_{\mathrm{g} 1}=\max \left(\mathrm{n}_{\mathrm{g} 11}, \mathrm{n}_{\mathrm{g} 12}=250\right.$

- $\mathrm{n}_{\mathrm{g} 2}=\max \left(\mathrm{n}_{\mathrm{g} 21}, \mathrm{n}_{\mathrm{g} 22}=250\right.$

- $\mathrm{T}=2$

Al igual que en el apartado 5.7 se va a comparar el ajuste obtenido con el método propuesto con los parámetros calculados mediante Shridhar-Cooper. Para ellos es necesario calcular el equivalente FOPDT de la columna de destilación a partir de la respuesta a escalón del sistema.

$$
\mathbf{G}=\left(\begin{array}{cc}
\frac{1,598}{(109 \mathrm{~s}+1)} & \frac{-1,580 \mathrm{e}^{-20 \mathrm{~s}}}{(110 \mathrm{~s}+1)} \\
\frac{0,0847}{(24 \mathrm{~s}+1)} & \frac{-0,0865}{(23 \mathrm{~s}+1)}
\end{array}\right)
$$

Empleando los datos de la Ecuación 5.31 en las Ecuaciones 5.8 a 5.11, se obtienen los siguientes valores de ajuste:

- $\operatorname{Pr}_{1}=\mathrm{n}_{\mathrm{g} 1}=286$

- $\operatorname{Pr}_{2}=\mathrm{n}_{\mathrm{g} 2}=286$

- $\lambda_{1}=3,14$

- $\lambda_{1}=2,89$

- $\mathrm{M}_{1}=\mathrm{M}_{2}=3$ 


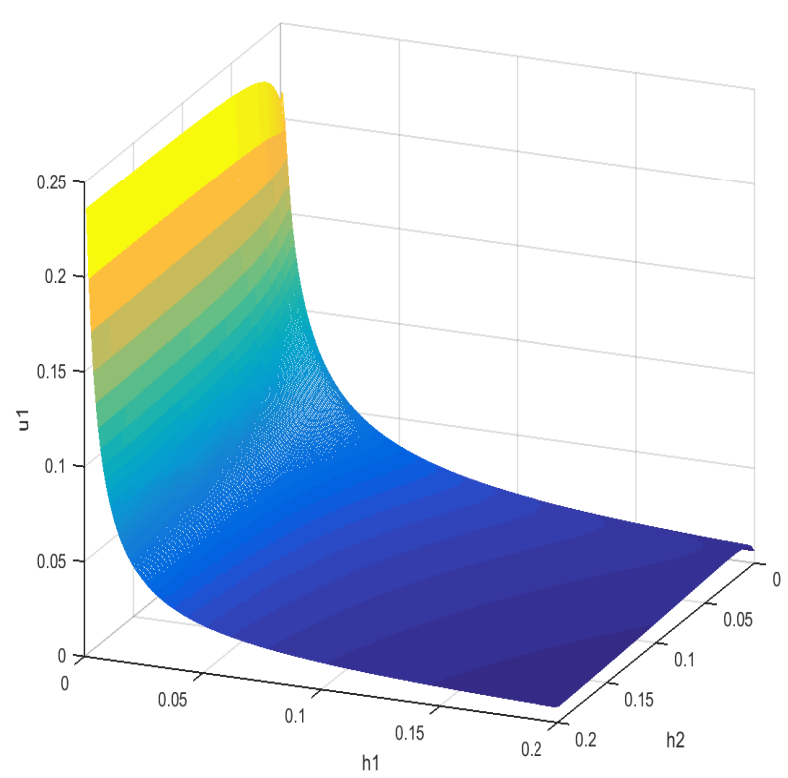

Curva $\mathrm{u}_{1}$

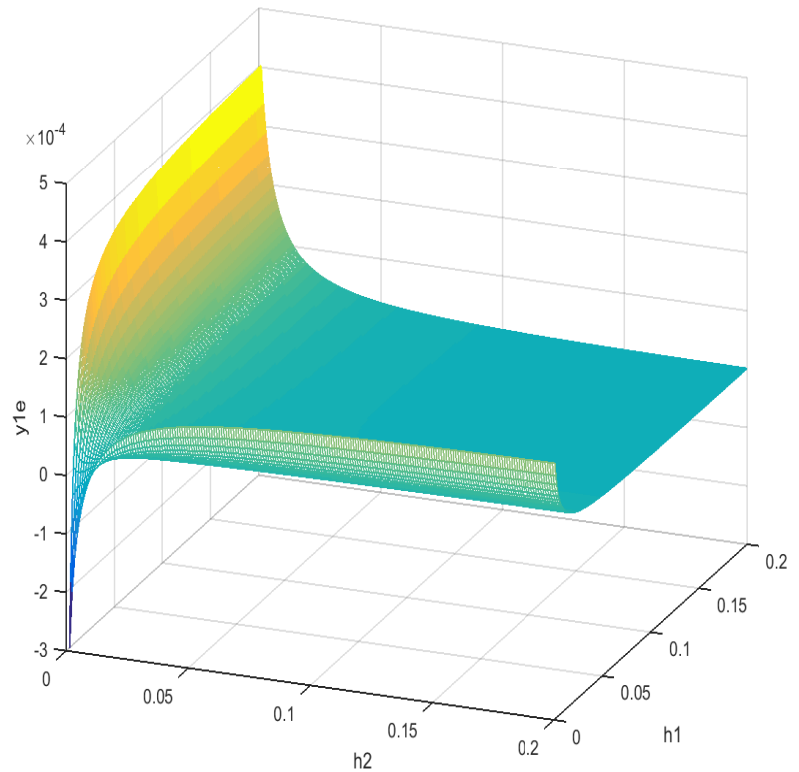

Curva $\mathrm{y}_{1}$

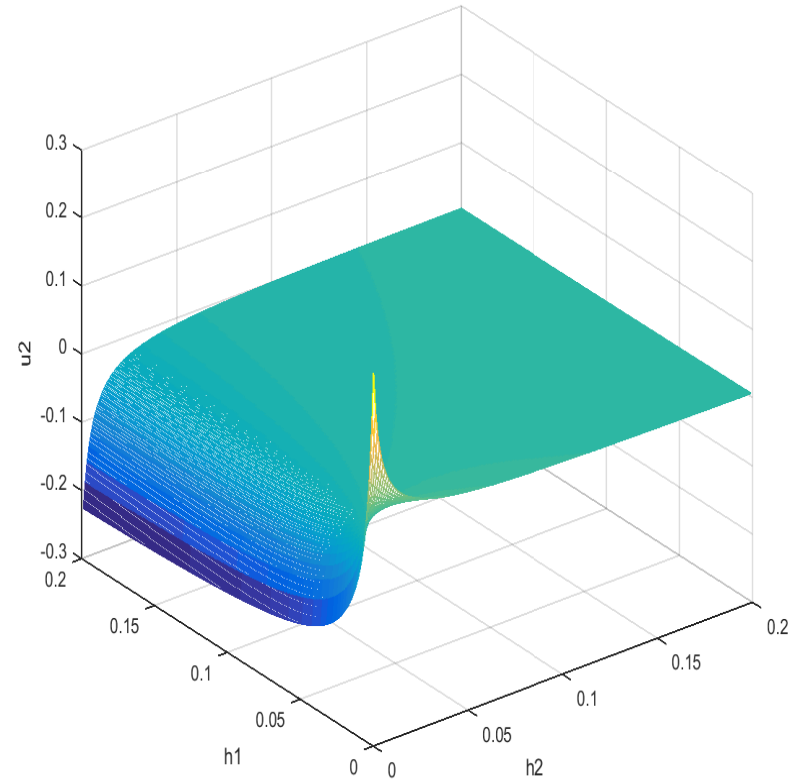

Curva $\mathrm{u}_{2}$

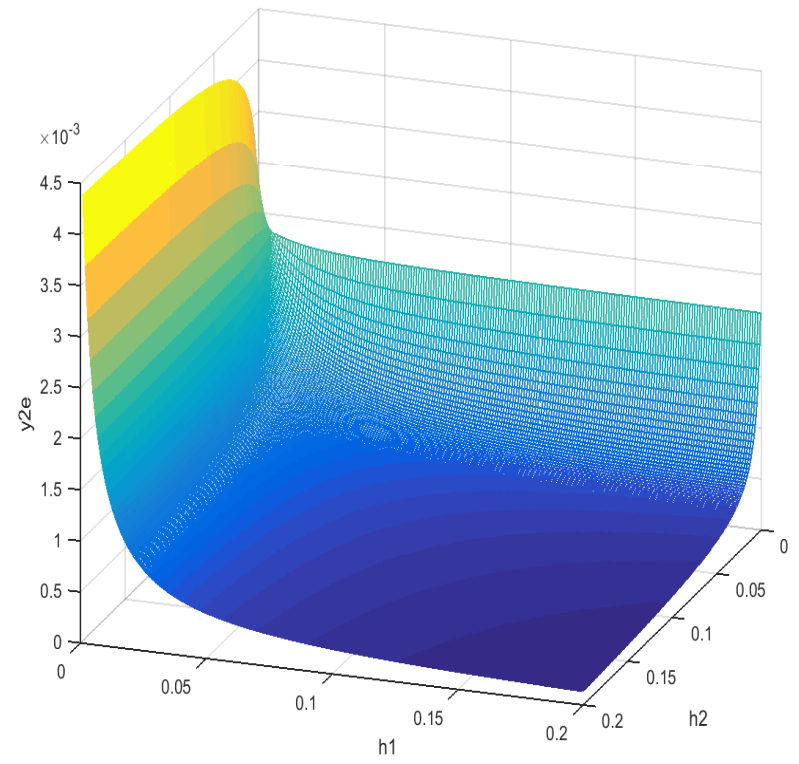

Curva $\mathrm{y}_{2}$

Figura 5.28: Curvas de primer incremento de control y salida para la columna de destilación 
- $\mathrm{T}=2$

Los resultados de ambos ajustes se muestran en la Figura 5.29 y el Cuadro 5.9 el error cuadrático medio de ambos métodos respecto a la referencia. La Figura 5.29 compara la respuesta temporal de la columna de destilación para ambos métodos de sintonía. Como puede verse, ambos ajustes consiguen pasar de un punto de funcionamiento a otro con éxito. Sin embargo el método propuesto consigue manejar mejor el caudal del calentador.

\begin{tabular}{|c|c|c|}
\hline Salida & Método propuesto & Método Shridhar Cooper \\
\hline $\mathrm{y}_{1}$ & $1,34 \mathrm{e}-4$ & $3,77 \mathrm{e}-4$ \\
\hline $\mathrm{y}_{2}$ & $1,31 \mathrm{e}-6$ & $1,77 \mathrm{e}-5$ \\
\hline
\end{tabular}

Cuadro 5.9: Error cuadrático medio para cada método

\subsection{Análisis de resultados}

En este capítulo se ha conseguido adaptar las reglas de diseño para sistemas SISO del capítulo 3 para sistemas MIMO. Se ha demostrado la utilidad de las mismas:

- Sintonizando varios benchmark representativos ([54],[55], [56])

- Comparando el método con un método reconocido [17]

- Ajustando el control de DMC de un modelo conocido de la literatura, con un comportamiento cercano a un modelo real.

Al igual que en el capítulo 3, estas reglas se han obtenido con sistemas de primer orden con retardo, por lo que pueden emplearse con cualquier sistema que pueda aproximarse mediante un FOPDT. 

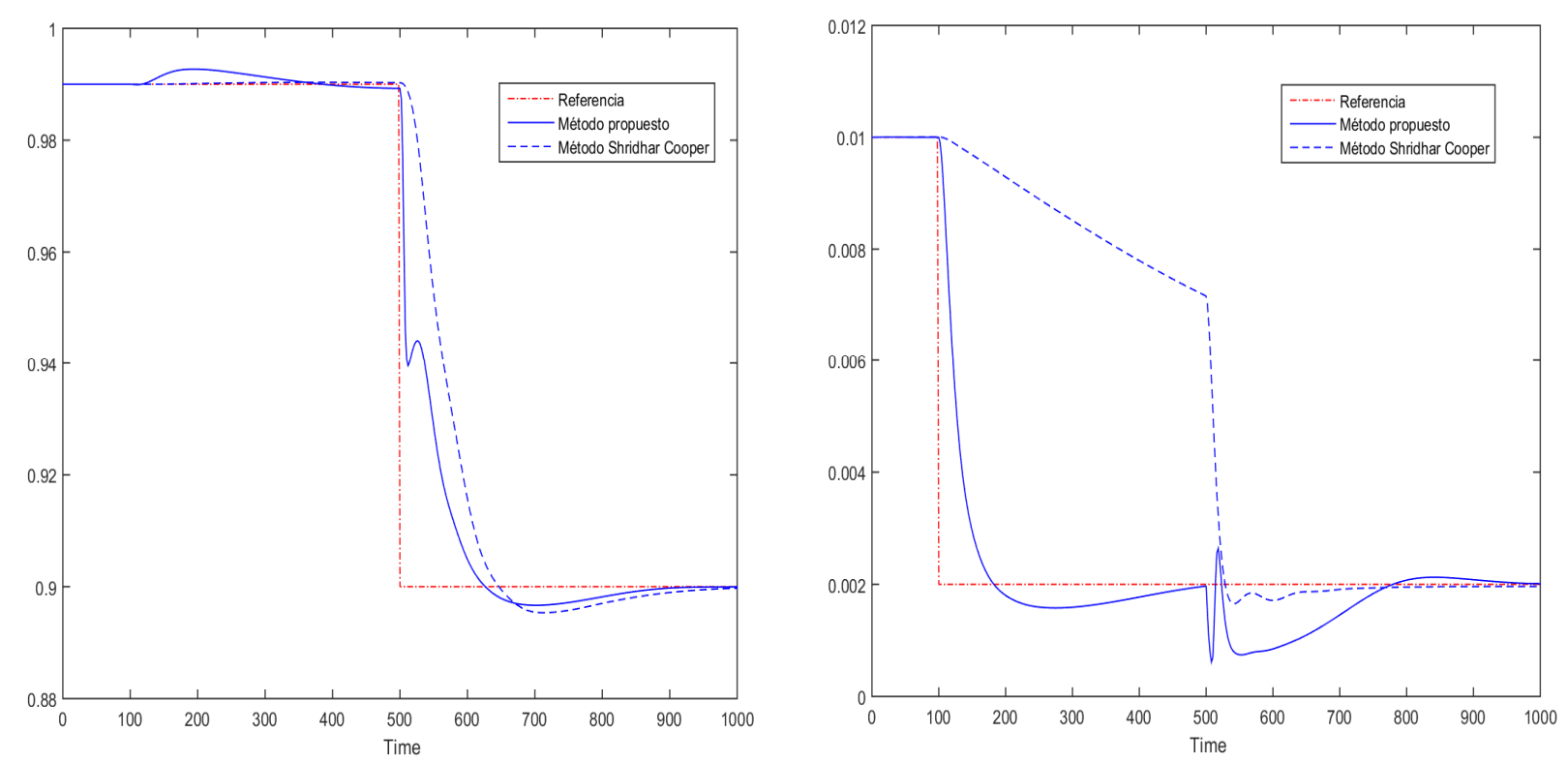

$\mathrm{y}_{1}$

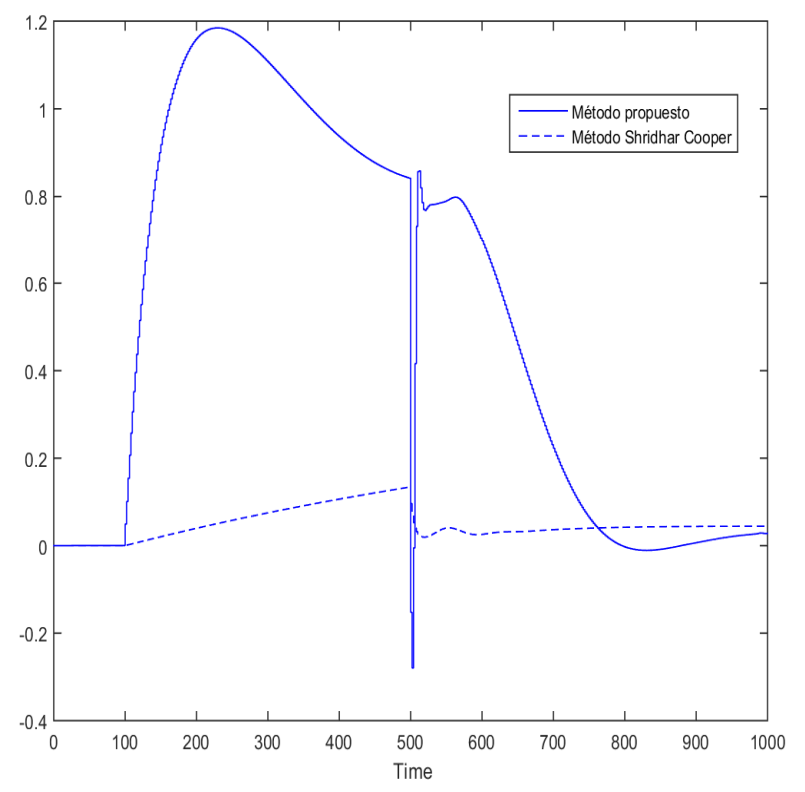

$\mathrm{u}_{1}$

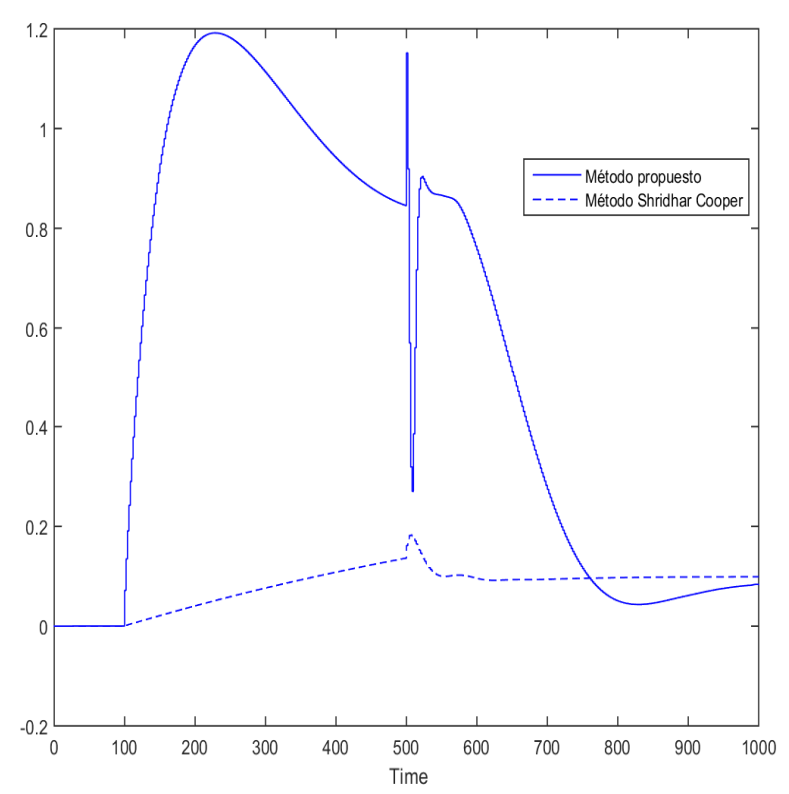

$u_{2}$

Figura 5.29: Resultado de sintonía para la columna de destilación 


\section{Capítulo 6}

\section{Conclusiones}

Considerando los resultados obtenidos a lo largo del presente trabajo, puede concluirse que se han alcanzado los objetivos fijados en el capítulo 1:

1. Se ha formulado el DMC SISO como un sistema LTI, basándose en un desarrollo existente en la literatura.

2. Se han definido unas reglas de sintonía para SISO basadas en el desplazamiento de los polos en lazo cerrado, fáciles de comprender y aplicar que posibilitan unas prestaciones que mejoran los métodos existentes.Dichas reglas se han obtenido mediante el estudio de que pueden ser reducidos a un FOPDT. De esta forma se reduce el número de polos (haciendo más sencillo el análisis) y se asegura su validez para todo aquel sistema que pueda ser simplificado mediante un FOPDT.

3. Se ha desarrollado una formulación que permite expresar el algoritmo del DMC MIMO como un sistema de bloques LTI.Dicho sistema de bloques se puede simplificar para obtener las relaciones directas $\left(\mathrm{H}_{\mathrm{xx}}, \mathrm{H}_{\mathrm{yy}}\right)$ e indirectas $\left(\mathrm{H}_{\mathrm{xy}}, \mathrm{H}_{\mathrm{yx}}\right)$, lo que permite calcular los polos del sistema. Esta nueva formulación se ha puesto a prueba comparando los resultados del algoritmo DMC clásico con el equivalente LTI. En todos los casos, la respuesta temporal coincidía para ambas formulaciones, demostrando la validez del método. La utilidad de este equivalente ha quedado evidenciada al permitir aplicar herramientas clásicas del análisis de sistemas al DMC y demostrar su efecto como desacoplador.

4. Utilizando la formulación LTI se han conseguido calcular los polos en lazo cerrado y la matriz RGA del sistemas DMC MIMO. Esto ha hecho posible ampliar las reglas obtenidas para problemas SISO a sistemas MIMO, que son los más frecuentes en la práctica, pero para los que es más complicado encontrar trabajos dedicados a su ajuste. El método obtenido se basa en el estudio del efecto de cada parámetro en los polos y en la respuesta temporal, por lo que no es necesario poseer fuertes conocimientos matemáticos y solo conocimientos básicos de teoría de control para 
comprenderlo y aplicarlo, que es lo que se buscaba: Un método sencillo, en la línea de los métodos experimentales ya establecidos para PID a nivel industrial.

5. Se ha comprobado la eficacia de dichas reglas mediante simulación en benchmark extraídos de la bibliografía y anteriormente empleados para el desarrollo de reglas de sintonía para MPC.

6. La comprobación final del método se ha llevado a cabo diseñando un controlador DMC de una maqueta real y mediante simulación de un conocido modelo no lineal con un comportamiento cercano al real.

7. Las simulaciones anteriores se han empleado para comparar el método propuesto con otras técnicas existentes y confirmar que mejora sus prestaciones.

8. La formulación LTI conseguida en esta tesis no es una herramienta que solo pueda utilizarse para el desarrollo de reglas de sintonía. La posibilidad de expresar el algoritmo del DMC (tanto MIMO como SISO) en forma de sistema de bloques LTI y que permita el uso de técnicas de análisis de teoría de sistemas posibilita futuros estudios de robustez y estabilidad. 


\section{Apéndice A}

\section{Simplificaciones para formulación LTI MIMO}

En este apéndice se explicarán las simplificaciones hechas para obtener unas ecuaciones fáciles de usar para a partir de la Ecuación 4.42

Los polinomios $A_{i j}$ y $B_{i j}$ agrupan los términos que multiplican a la referencia o la salidad medida en la ecuación 4.43. los polinomios $\mathrm{A}_{\mathrm{ij}}$ se convierten en escalares cuando la referencia es una constante $(\omega(\mathrm{t})=\omega(\mathrm{t}+1)=\ldots=\omega(\mathrm{t}+\mathrm{k}))$ y toma el mismo valor que $\mathrm{B}_{\mathrm{ij}}$. A pesar de esto, mantendrán la misma designación al tener una posición distinta en la Figura 4.5 y no compartir el mismo origen.

$$
\begin{aligned}
A_{i j} & =\sum_{j=\operatorname{Pr}_{1}+\ldots+\operatorname{Pr}_{j-1}+1}^{\operatorname{Pr}_{1}+\ldots+\operatorname{Pr}_{j}} \phi_{1 j} \\
B_{i j} & =\sum_{j=\operatorname{Pr}_{1}+\ldots+\operatorname{Pr}_{j-1}+1}^{\operatorname{Pr}_{1}+\ldots+\operatorname{Pr}_{j}} \phi_{1 j}
\end{aligned}
$$

El resto de términos $(\mathrm{C}, \mathrm{D}, \ldots, \mathrm{Z})$ son familias de polinomios en $\mathrm{z}^{-1}$ y agrupan todos los términos que multiplican los incrementos de control pasados de la respuesta libre. Hay una familia de polinomios para cada variable controlada y cada familia tiene tantos polinomios como variables manipuladas. Por ejemplo, tomemos como ejemplo el caso [4x4]. Habrá cuatro familias de polinomios $(\mathrm{C}, \mathrm{D}, \mathrm{E}, \mathrm{F})$ y cada familia tendrá cuatro polinomios:

La primera familia (Correspondiente a la primera variable manipulada) secalcula de la siguiente manera:

$$
\mathrm{C}_{1}=\sum_{\mathrm{i}=1}^{\mathrm{z}}\left(\sum_{\mathrm{j}=1}^{\operatorname{Pr}_{1}} \phi_{1 \mathrm{j}} \mathrm{S}_{\mathrm{n}, 1 \mathrm{i}}^{\mathrm{z}}\left(\mathrm{q}^{-1}\right)\right.
$$




\begin{tabular}{|c|c|c|c|}
\hline \multicolumn{4}{|c|}{$\mathrm{C}$} \\
\hline $\mathrm{C}_{1}$ & $\mathrm{C}_{2}$ & $\mathrm{C}_{3}$ & $\mathrm{C}_{4}$ \\
\hline \multicolumn{4}{|c|}{$\mathrm{D}$} \\
\hline $\mathrm{D}_{1}$ & $\mathrm{D}_{2}$ & $\mathrm{D}_{3}$ & $\mathrm{D}_{4}$ \\
\hline \multicolumn{4}{|c|}{$\mathrm{E}$} \\
\hline $\mathrm{E}_{1}$ & $\mathrm{E}_{2}$ & $\mathrm{E}_{3}$ & $\mathrm{E}_{4}$ \\
\hline \multicolumn{4}{|c|}{$\mathrm{F}$} \\
\hline $\mathrm{F}_{1}$ & $\mathrm{~F}_{2}$ & $\mathrm{~F}_{3}$ & $\mathrm{~F}_{4}$ \\
\hline
\end{tabular}

Cuadro A.1: Simplificaciones para sistemas de orden superior a [2x2]

$$
\begin{gathered}
\mathrm{C}_{2}=\sum_{\mathrm{i}=1}^{\mathrm{z}}\left(\sum_{\mathrm{j}=\operatorname{Pr}_{1}+1}^{\operatorname{Pr}_{1}+\operatorname{Pr}_{2}} \phi_{1 \mathrm{j}} \mathrm{S}_{\mathrm{n}, 2 \mathrm{i}}^{\mathrm{j}-\operatorname{Pr}_{1}+1}\left(\mathrm{q}^{-1}\right)\right. \\
\cdot \\
\mathrm{C}_{\mathrm{z}}=\sum_{\mathrm{i}=1}^{\mathrm{z}}\left(\sum_{\mathrm{j}=\operatorname{Pr}_{1}+\ldots+\operatorname{Pr}_{\mathrm{z}-1}+1}^{\operatorname{Pr}_{1}+\ldots+\operatorname{Pr}_{\mathrm{z}}} \phi_{1 \mathrm{j}} \mathrm{S}_{\mathrm{n}, \mathrm{zi}}^{\mathrm{j}-\operatorname{Pr}_{1}+\ldots+\operatorname{Pr}_{\mathrm{z}-1}+1}\left(\mathrm{q}^{-1}\right)\right.
\end{gathered}
$$

$\mathrm{Y}$ generalizando a $\mathrm{z}$ variables:

$$
\begin{gathered}
\mathrm{Z}_{1}=\sum_{\mathrm{i}=1}^{\mathrm{z}}\left(\sum_{\mathrm{j}=1}^{\operatorname{Pr}_{1}} \phi_{1 \mathrm{j}} \mathrm{S}_{\mathrm{n}, 1 \mathrm{i}}^{\mathrm{z}}\left(\mathrm{q}^{-1}\right)\right. \\
\mathrm{Z}_{2}=\sum_{\mathrm{i}=1}^{\mathrm{z}}\left(\sum_{\mathrm{j}=\operatorname{Pr}_{1}+1}^{\operatorname{Pr}_{1}+\operatorname{Pr}_{2}} \phi_{1 \mathrm{j}} \mathrm{S}_{\mathrm{n}, 2 \mathrm{i}}^{\mathrm{j}-\mathrm{Pr}_{1}+1}\left(\mathrm{q}^{-1}\right)\right. \\
\cdot \\
\cdot \\
\mathrm{Z}_{\mathrm{z}}=\sum_{\mathrm{i}=1}^{\mathrm{z}}\left(\sum_{\mathrm{j}=\operatorname{Pr}_{1}+\ldots+\operatorname{Pr}_{\mathrm{z}-1}+1}^{\operatorname{Pr}_{1}+\ldots+\operatorname{Pr}_{\mathrm{z}}} \phi_{1 \mathrm{j}} \mathrm{S}_{\mathrm{n}, \mathrm{zi}}^{\mathrm{j}-\operatorname{Pr}_{1}+\ldots+\operatorname{Pr}_{\mathrm{z}-1}+1}\left(\mathrm{q}^{-1}\right)\right.
\end{gathered}
$$




\section{Índice de figuras}

2.1. Evolución histórica del MPC en la industria . . . . . . . . . . . . . . 9

2.2. Estrategia MPC . . . . . . . . . . . . . . . . . 15

2.3. Estructura básica MPC . . . . . . . . . . . . . . . . . 15

3.1. DMC con formulación LTI . . . . . . . . . . . . . . . . . . . . . 28

3.2. DMC SISO como sistema en lazo abierto . . . . . . . . . . . . . . 29

3.3. Respuesta a un polo simple real estable positivo (a) y negativo (b) . . . . . 31

3.4. Respuesta a una pareja de polos complejos estables con parte real positiva

(a) y negativa $(b)(c) \ldots \ldots \ldots . \ldots \ldots . \ldots \ldots$

3.5. Localización de polos y respuesta temporal para $\mathrm{T}=8$ y $\mathrm{T}=16$ segundos . . 34

3.6. Localización de polos y respuesta temporal para diferentes valores de M . . 37

3.7. Localización de polos y respuesta temporal variando $\operatorname{Pr}$ y M . . . . . . . . 38

3.8. Localización de polos y respuesta temporal para distintos valores de $\lambda$. . . 39

3.9. Curva de incrementos de control-factor de peso . . . . . . . . . . . 40

3.10. Determinante de $\mathrm{G}^{\mathrm{T}} \mathrm{G}$ para $\lambda=0 \ldots \ldots \ldots \ldots . \ldots \ldots 41$

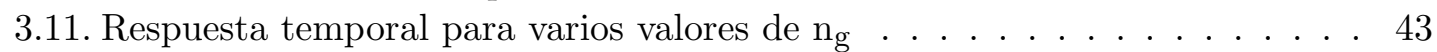

3.12. Polos para $\lambda=0 \mathrm{Pr}=100$ y $\lambda=0.005$ y $\mathrm{M}=2 \ldots \ldots \ldots$. . . . . 44

3.13. Polos para $\lambda=0.075 \ldots \ldots \ldots \ldots$. . . . . . . . . . 46

3.14. (a) Respuesta a escalón, (b) Curva de factor de peso (c) Respuesta temporal para cada benchmark dependiendo del valor de $\lambda \ldots$. . . . . . . . . . 49

3.15. (a) Respuesta a escalón, (b) Curva de factor de peso (c) Respuesta temporal Respuesta temporal cada benchmark dependiendo del valor de $\lambda$. . . . . 50

3.16. Comparación de resultados de los distintos métodos de ajuste en los bench-

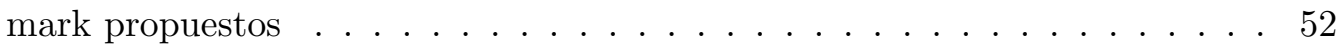

3.17. Maqueta térmica . . . . . . . . . . . . . . . 54

3.18. Diagrama bloques maqueta térmica . . . . . . . . . . . . . . 54

3.19. Curvas de definición de la maqueta térmica . . . . . . . . . . . . 55

3.20. Resultados maqueta térmica. La línea continua indica la referencia y la discontinua la respuesta del sistema . . . . . . . . . . . . . . . . 57

4.1. Representación en funciones de transferencia de un sistema MIMO lineal 2x2 59

4.2. Sistema MIMO 2x2 controlado usando lazos de control SISO . . . . . . . 60

4.3. Sistema controlado por DMC . . . . . . . . . . . . . . . . 61

4.4. Representación típica MIMO LTI usando notación matricial . . . . . . . . . 61 
4.5. Representación LTI de un sistema controlado mediante DMC . . . . . . . . 67

4.6. Representación LTI simplificada de un sistema controlado mediante DMC . 69

4.7. MIMO DMC en forma de sistema en lazo abierto . . . . . . . . . . . . 70

4.8. Comparación de métodos para el benchmark $1 \ldots \ldots$. . . . . . . 74

4.9. Comparación de métodos para el benchmark $2 \ldots \ldots$. . . . . . 75

4.10. Comparación de métodos para el benchmark $3 \ldots \ldots$. . . . . . 76

4.11. Benchmark 1 Salidas para el conjunto de parámetros $2 \ldots \ldots$. . . . . . 78

4.12. Benchmark 2 Salidas para el conjunto de parámetros 2 . . . . . . . . . . 79

4.13. Benchmark 3 Salidas para el conjunto de parámetros 1 . . . . . . . . . 79

5.1. Evolución de los polos variando $\operatorname{Pr}_{1}$ para un valor fijo de $\operatorname{Pr}_{1}=2 \ldots$. . . 83

5.2. Evolución de los polos variando $\operatorname{Pr}_{2}$ para un valor fijo de $\operatorname{Pr}_{2}=2 \ldots$. . . 84

5.3. Evolución de la respuesta variando $\operatorname{Pr}_{1}$ para un valor fijo de $\operatorname{Pr}_{2}=2 \ldots$. . 85

5.4. Evolución de la respuesta variando $\operatorname{Pr}_{2}$ para un valor fijo de $\operatorname{Pr}_{1}=2 \ldots$. . 85

5.5. Evolución de los polos variando $\mathrm{M}_{1}$ para un valor fijo de $\mathrm{M}_{2}=1 \ldots$. . . 86

5.6. Evolución de los polos variando $\mathrm{M}_{2}$ para un valor fijo de $\mathrm{M}_{1}=1 \ldots$. . . 87

5.7. Evolución de la respuesta variando $\mathrm{M}_{1}$ para un valor fijo de $\mathrm{M}_{2}=1 \ldots$. . 88

5.8. Evolución de la respuesta variando $\mathrm{M}_{2}$ para un valor fijo de $\mathrm{M}_{1}=1 \ldots$. . 88

5.9. Evolución de los primeros incrementos de control con los factores de peso . 89

5.10. Evolución de los primeros valores de salida con los factores de peso . . . . . 90

5.11. Evolución de los polos variando $\lambda_{2}$ para un valor fijo de $\lambda_{1}=0,1 \ldots \ldots 1$

5.12. Evolución de los polos variando $\lambda_{1}$ para un valor fijo de $\lambda_{2}=0,1 \ldots$. . . 92

5.13. Evolución de la respuesta variando $\lambda_{1}$ para un valor fijo de $\lambda_{2}=0,1 \ldots$. . 93

5.14. Evolución de la respuesta variando $\lambda_{2}$ para un valor fijo de $\lambda_{1}=0,1 \ldots$. . 93

5.15. Evolución de $\mathrm{u}_{1} \mathrm{y}_{2}$ variando $\lambda_{1} \mathrm{y}_{2} \ldots \ldots \ldots \ldots$. . . . . . . . . 95

5.16. Evolución de $\mathrm{u}_{1} \mathrm{y} \mathrm{u}_{2}$ variando $\lambda_{1} \mathrm{y} \lambda_{2}$ para benchmark $1 \ldots \ldots$. . . . . . 98

5.17. Resultado de sintonía para benchmark 1 . . . . . . . . . . . . . . 100

5.18. Resultado de sintonía para benchmark $2 \ldots \ldots$. . . . . . . . 101

5.19. Evolución de $\mathrm{u}_{1} \mathrm{y}_{2}$ variando $\lambda_{1} \mathrm{y} \lambda_{2}$ para benchmark $3 \ldots \ldots$. . . . . . 103

5.20. Resultado de sintonía para benchmark $3 \ldots \ldots$. . . . . . . . . . 104

5.21. Comparativa para benchmark $1(\mathrm{~T}=3) \ldots \ldots \ldots \ldots 7 . \ldots \ldots$

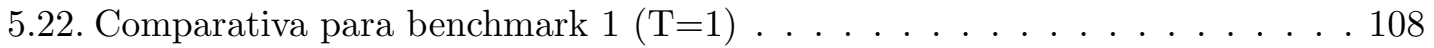

5.23. Comparativa para benchmark $2 \ldots \ldots$. . . . . . . . . . 109

5.24. Comparativa para benchmark $3 \ldots \ldots$. . . . . . . . . . 110

5.25. Diagrama de la columna de destilación de alta pureza . . . . . . . . . . . . . 113

5.26. Respuesta escalón columna destilación . . . . . . . . . . . . . . . 117

5.27. Modelo de respuesta escalón columna destilación . . . . . . . . . . . . 118

5.28. Curvas de primer incremento de control y salida para la columna de destilación 120

5.29. Resultado de sintonía para la columna de destilación . . . . . . . . . . . . 122 


\section{Índice de cuadros}

2.1. Productos representativos de control predictivo . . . . . . . . . . . 23

2.2. Aplicaciones industriales del control predictivo . . . . . . . . . . . 23

3.1. Polos para $\mathrm{T}=8$ segundos . . . . . . . . . . . . . . . . 35

3.2. Polos for $\mathrm{T}=16$ segundos . . . . . . . . . . . . . . . 35

3.3. Polos para diferentes valores de $\mathrm{M} \ldots \ldots \ldots$. . . . . . . . . 36

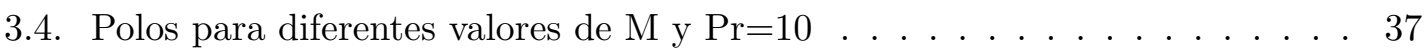

3.5. Polos para diferentes valores de $\mathrm{M} \mathrm{y} \mathrm{Pr}=60 \ldots \ldots$. . . . . . . . . . . . . . . . . . . . 37

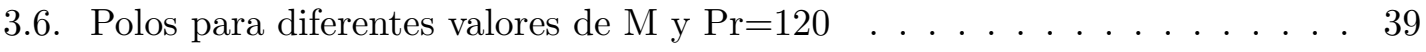

3.7. Evolución de polos con $\lambda \ldots \ldots \ldots \ldots$. . . . . . . . . . 42

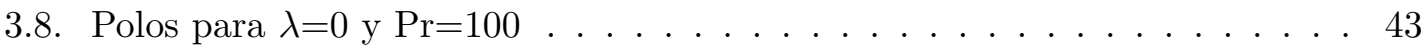

3.9. Polos para $\lambda=0.005$ y $\mathrm{M}=2 \ldots \ldots \ldots \ldots \ldots 4$

3.10. Polos para $\lambda=0.075, \mathrm{Pr}=100$ variando M . . . . . . . . . . . 45

3.11. Polos para $\lambda=0.075, \mathrm{M}=2$ variando $\operatorname{Pr} \ldots \ldots \ldots \ldots$. . . . . . . 45

3.12. Resultados del ajuste para cada benchmark . . . . . . . . . . . . . 48

3.13. Parámetros según los distintos métodos para cada uno de los benchmark . . 53

3.14. Error cuadrático medio para cada método . . . . . . . . . . . . 53

3.15. Resultados maqueta térmica . . . . . . . . . . . . . . . 56

4.1. Simplificaciones . . . . . . . . . . . . . . . . . 65

4.2. Conjunto de parámetros para Benchmark $1 \ldots \ldots$. . . . . . . . . 73

4.3. Conjunto de parámetros para Benchmark $2 \ldots \ldots$. . . . . . . . 73

4.4. Conjunto de parámetros para Benchmark $3 \ldots \ldots$. . . . . . . . 73

5.1. Resultados del ajuste para benchmark $1 \ldots \ldots$. . . . . . . . . . 97

5.2. Resultados del ajuste para benchmark $2 \ldots \ldots$. . . . . . . . . . 99

5.3. Resultados del ajuste para cada benchmark 3 . . . . . . . . . . . . . . . 102

5.4. Resultados del ajuste usando el método de Shridhar Cooper . . . . . . . . . 106

5.5. Error cuadrático medio para cada método . . . . . . . . . . . . . . 106

5.6. Datos columna de destilación . . . . . . . . . . . . . . . . . . . 114

5.7. Puntos de funcionamiento columna de destilación . . . . . . . . . . . . . . . 114

5.8. Resultados del ajuste para columna de destilación . . . . . . . . . . . . 116

5.9. Error cuadrático medio para cada método . . . . . . . . . . . . . . . . 121 
A.1. Simplificaciones para sistemas de orden superior a $[2 \mathrm{x} 2] \ldots \ldots$. . . . . 126 


\section{Bibliografía}

[1] S. Qin and T. A Badgwell. A survey of industrial model predictive control technology. Control Engineering Practice, 11:733-764, 2003.

[2] Ziegler J.G and N.B Nichols. Optimun settings for automatic controllers. Trans ASMEl, pages 759-768, 1942.

[3] J.Richalet, A.Rault, J.L Testud, and J.Papon. Algorithmic Control of Industrial Processes. In 4th IFAC Symposium on Identification and System Parameter Estimation, 1976 .

[4] J.Richalet, A.Rault, J.L Testud, and J.Papon. Model Predictive Heuristic Control: Application to Industrial Processes. Automatica, 14:413-418, 1978.

[5] C. R. Cutler and B. L. Ramaker. Dynamic Matrix Control- a Computer Control Algorithm. In Proceedings of Joint Automatic Control Conference, 1980.

[6] Rickey Dubay, Guy Kember, and Bambang Pramujati. Well-conditioned model predictive control. ISA Transactions, 43:23-32, 2004.

[7] R. Dubay, G. Kember, C.V. Lakshminarayan, and B. Pramujati. Development of characteristic equations and robust stability analysis for MIMO move suppressed and shifted dmc. ISA Transactions, 44:465-479, 2005.

[8] Jonathan R. Webber and Yash P. Gupta. Robust and nominal stability conditions for a simplified model predictive controller. ISA Transactions, 45:77-86, 2006.

[9] E. Mosca D.W Clarke and R.Scattolini. Constrained Receding-Horizon Predictive Control. Proceedings IEE, 138:347-354, 1991.

[10] J.M Lemos E.Mosca and J.Zhang. Stabilizing I/O Receding Horizon Control. In IEEE Conference on Decision and Control, 1990.

[11] J.A Rossiter B.Kouvaritakis and A.O.T Chang. Stable Generalized Predictive Control: An Algorithm with Guaranteed Stability. Proceedings IEE, 139:349-362, 1992.

[12] N. L. Ricker. Model Predictive Control: State of the Art. In Proc.Chem. Process Control Conf., CPC IV, Fourth International Conference on Chemical Process Control; Arkun, Y., Ray, W.H., Eds.; Elsevier: Amsterdam, Holland, 1983. 
[13] J. L. Marchetti, D. A. Mellichamp, and D. E. Seborg. Predictive Control Based on Discrete Convolution Models. Ind. Eng.Chem. Process Des. Dev, 22:488, 1983.

[14] E.F Camacho and C. Bordons. Model Predictive Control. Springer, 2 edition, 2003.

[15] Javier Serrano. Aspectos de implementacion y analisis de robustez en CPBM. PhD thesis, Universidad Autonoma de Barcelona, 1994.

[16] Rahul Shridhar and Douglas J. Cooper. A Tuning Strategy for Unconstrained SISO Model Predictive Control. Industrial and Enginnering Chemistry Research, 36:729746, 1997.

[17] Rahul Shridhar and Douglas J. Cooper. A Novel Tuning Strategy for Multivariable Model Predictive Control. ISA Transactions, 36:273-280, 1998.

[18] Edinzo J. Iglesias, Marco E. Sanjuan, and Carlos A. Smith. Tuning Equation for Dynamic Matrix Control in SISO Loops. Ingeniería y Desarrollo, 19:88-100, 2006.

[19] V.Peterka. Predictor Based Self Tuning Control. Automatica, 20:39-50, 1984.

[20] B.E Ydstie. Extended Horizon Adaptative Control. In 9th IFAC World Congress, 1984.

[21] R.M.C De Keyser and A.R Van Cuawenberghe. Extended Prediction Self Adaptative Control. In IFAC Symposium on Identification and System Parameter Estimation, 1985.

[22] Tamas Keviczky, Paolo Falcone, Francesco Borrelli, Jahan Asgari, and Davor Hrovat. Predictive Control Approach to Autonomous Vehicle Steering. In American Control Conference, 2006.

[23] Frank Allgöwer, Rolf Findeisen, and Zoltan K. Nagy. Nonlinear Model Predictive Control: From Theory to Application. Chemical Engineering Communications, 46:7687, 2004.

[24] R Kamyar and E. Taheri. Aircraft Optimal Terrain/Threat-Based Trajectory Planning and Control. Journal of Guidance, Control, and Dynamics, 37, 2014.

[25] Arthur Richards and Jonathan How. Performance evaluation of rendezvous using Model Predictive Control. In Navigation, and Control Conference and Exhibit11-14 August 2003, Austin, Texas, 2003.

[26] J.Gomez Ortega and E.F.Camacho. Mobile robot navigation in a partially structured environment using neural predictive control. Control Engineering Practice, 4:16691679, 1996.

[27] D.A.Linkers and M.Mahfonf. Advances in model based predictive control. Oxford University Press, 1 edition, 1994. 
[28] R.E Kalman. Contribution to the theory of optimal control. Bull. Soc.Math. Mex, 5:102-119, 1960.

[29] R.E Kalman. A new approach to linear filtering and prediction problems. Trans. ASME, J. Basic Engineering, pages 35-45, 1960.

[30] H. Kwakernaak and R. Sivan. Linear optimal control systemsl. Wiley, 1 edition, 1972.

[31] G. C. Goodwin, S. F. Graebe, and Salgado. Control system design. Englewood Cliffs, NJ: Prentice Hall., 1 edition, 2001.

[32] D. M. Prett and R. D.. Gillette. Optimization and constrained multivariable control of a catalytic cracking unit. In Proceedings of the joint automatic control conference, 1980 .

[33] C. R. Cutler and B. L. Ramaker. Dynamic Matrix Control- a Computer Control Algorithm. In AICHE national meeting, Houston, TX, 1979.

[34] C. Cutler, A. Morshedi, and J Haydel. An industrial perspective on advanced control. In AICHE annual meeting, Washington DC, 1983.

[35] C. E. García and A. M Morshedi. Quadratic programming solution of dynamic matrix control (QDMC). Chemical Engineering Communications, 46:76-87, 1986.

[36] D. M. Prett and C. E. García. Fundamental process control. Boston: Butterworths., 1 edition, 1988.

[37] S. Qin and T. A Badgwell. An Overview of Industrial Model Predictive Control Technology. AIChe Symposium Series, pages 232-236, 2003.

[38] A. Ahmad and A. Wahid. Application of model predictive control (MPC) tuning strategy in multivariable control of distillation column. Reaktor, 11:66-70, 2007.

[39] R D Kokate and L M Waghmare. Review of Tuning Methods of DMC and Performance Evaluation with PID Algorithms on a FOPDT Model. International Journal of Control and Automation, 4, 2011.

[40] J.O. Trierweiler and L.A. Farina. RPN Tuning Strategy for Model Predictive Control. Journal of Process Control, 13:591-598, 2003.

[41] Kai Han, Jun Zhao, and Jixin Qian. A Novel Robust Tuning Strategy for Model Preditive Control. In The Sixth World Congress on Intelligent Control and Automation, 2006 .

[42] Jorge L. Garriga and Masoud Soroush. Model Predictive Controller Tuning via Eigenvalue Placement. In American Control Conference, 2008.

[43] Oscar Camacho, Edinzo Iglesias, Luís Valverde, and Francklin Rivas. An approach to enhance dynamic matrix control performance. International Journal of Mathematics and Computers in Simulation, 2(1):81-88, 2008. 
[44] Peyman Bagheri and Ali Khaki-Sedigh. Tuning of Dynamic Matrix Controller for FOPDT models using analysis of variance. In 18th IFAC World Congress, 2011.

[45] Willy Wojsznis, John Gudaz, Terry Blevins, and Ashish Mehta. Practical approach to tuning MPC. ISA Transactions, 42:149-162, 2003.

[46] G-Valencia-Palomo and J.A Rossiter. Auto Tuned Predictive Control Based on Minimal Plant Information. In 7th IFAC International Symposium on Advanced Control of Chemical Processes, 2009.

[47] J.H. van der Lee, W.Y. Svrcek, and B.R. Young. A tuning algorithm for model predictive controllers based on genetic algorithms and fuzzy decision making. ISA Transactions, 47:53-59, 2008.

[48] R Sivakumar, K Suresh Manic, V Nerthiga, R Akila, and K Balu. Application of fuzzy model predictive control in multivariable control of distillation column. International Journal of Chemical Engineering and Applications, 1(1):38, 2010.

[49] Rakesh Kumar Mishra, Rohit Khalkho, Brajesh Kumar, and Tarun Kumar Dan. Effect of tuning parameters of a model predictive binary distillation column. In Emerging Trends in Computing, Communication and Nanotechnology (ICE-CCN), 2013 International Conference on, pages 660-665. IEEE, 2013.

[50] R.C. Dorf. The Engineering Handbook, Second Edition. Electrical Engineering Handbook. CRC Press, 2004.

[51] J. Rawlings and K. Muske. The Stability of constrained Receding Horizon Control. IEEE Transactions On Automatic Control, 38, 1993.

[52] J.A Rossiter. Model-based predictive control: A practical approach. CRC Press LLC, 1 edition, 2003.

[53] Jie Sheng, Tongwen Chen, and Sirish L. Shah. Generalized Predictive Control for Non Uniformly Sampled Systems. Journal of Process Control, 2002.

[54] R.K. Wood and Berry M.W. Terminal composition of a binary distilation column. Chem. Eng. Sci., 28:1707-1717, 1973.

[55] P. Albertos, R.Strieztel, and N. Mort. Control Engineering Solutions: A Practical Approach. The Institution of Electrical Engineers, 1 edition, 1997.

[56] Manfred Morari and Evanghelos Zafiriou. Robust process control. Prentice-Hall, 1 edition, 1989.

[57] Edgar Bristol. On a new measure of interaction for multivariable process control. IEEE transactions on automatic control, 11(1):133-134, 1966.

[58] P.Albertos and A.Sala. Multivariable Control Systems. Springer, 1 edition, 2004. 
[59] Clemente Manzanera Reverter, Julio Ibarrola, and Jose-Manuel Cano-Izquierdo. Design rules for model predictive control using unconstrained Dynamic Matrix Control. In Seminario Anual de Electronica y Automatica Industrial, 2011.

[60] Clemente Manzanera Reverter, Julio Ibarrola, and Jose-Manuel Cano-Izquierdo. Tuning rules for a quick start up in Dynamic Matrix Control. ISA Transactions, 53:612627, 2014.

[61] Sigurd Skogestad and Manfred Morari. A systematic approach to distillation column control. Chemical Engineering, 206:41, 1987.

[62] Sigurd Skogestad and Manfred Morari. Understanding the dynamic behavior of distillation columns. Ind. Eng. Chem. Res, 27(10):1848-1862, 1988.

[63] Chih-An Hwang, James C. Holste, Kenneth Hall, and G. Ali Mansoori. A simple relation to predict or to correlate the excess functions of multicomponents mixtures. Fluid Phase Equilibria, 62:173-189, 1995.

[64] Smaranda Cristea, Almudena Rueda Ferreiro, and César de Prada. control predictivo no lineal aplicado a columnas de destilación de etanol. Universidad de Valladolid. Valladolid. España, 1999.

[65] Sigurd Skogestad. Dynamics and control of distillation columns-a critical survey. In IFAC Symposia Series, number 2, pages 11-35, 1993.

[66] Sigurd Skogestad. Dynamics and control of distillation columns: A tutorial introduction. Chemical Engineering Research and Design, 75(6):539-562, 1997.

[67] Natividad Duro Carralero and Fernando Morilla García. Modelado y simulación de columnas binarias de destilación con control de inventario. Workshop en: Metodologías de Modelado y Simulación de Sistemas, 2001., 2001.

[68] Sing Kiong Nguang and Xiao Dong Chen. Robust stabilization of binary distillation columns with input constraints. International Journal of Systems Science, 33(9):759$765,2002$.

[69] Clemente Manzanera Reverter, Julio Ibarrola, and Jose-Manuel Cano-Izquierdo. Linear time invariant formulation of MIMO systems controlled with DMC. Actualmente en proceso de segunda revision en la publicacion ISA Transactions. 


\section{Publicaciones}

El desarrollo de la presente tesis doctoral ha supuesto la publicación de los siguientes trabajos:

1. Clemente Manzanera Reverter, Julio Ibarrola and Jose-Manuel Cano-Izquierdo. Design rules for model predictive control using unconstrained Dynamic Matrix Control, Seminario Anual de Electrónica y Automática Industrial, 2011.[59]

2. Clemente Manzanera Reverter, Julio Ibarrola and Jose-Manuel Cano-Izquierdo. Tuning rules for a quick start up in Dynamic Matrix Control, ISA Transactions (Factor de impacto 2,6;Q1), 2014, Volumen 53, Enero 2014, Páginas 612-627. [60]

3. Clemente Manzanera Reverter, Julio Ibarrola and Jose-Manuel Cano-Izquierdo. Linear time invariant formulation of MIMO systems controlled with DMC. Actualmente en proceso de segunda revisión en la publicación ISA Transactions (Factor de impacto 2,6;Q1). [69] 
D. Julio José Ibarrola Lacalle y D. José Manel Cano Izquierdo, coautores del artículo científico con título "Tuning rules for a quick start up in Dynamic Matrix Control" publicado en la revista ISA Transactions, volume 53 pp 612-627, January 2014.

Exponen:

Que la aportación y el trabajo realizado por D. Clemente Manzanera Reverter en la elaboración de dicho trabajo científico ha sido fundamental.

$Y$, para que conste, a los efectos que procedan, firman el siguiente documento.

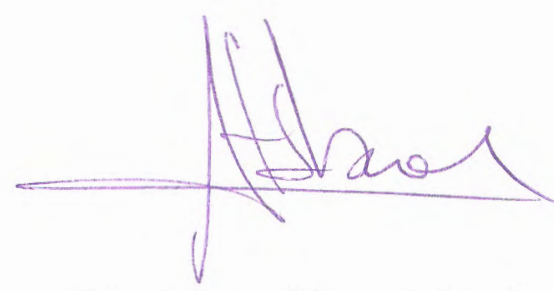

Fdo. Julio José Ibarrola Lacalle

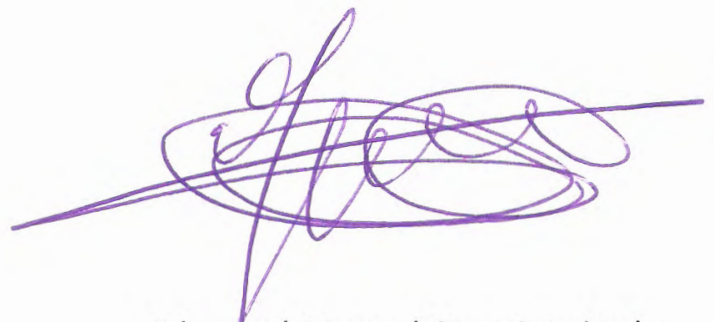

Fdo. José Manuel Cano Izquierdo 


\begin{tabular}{|l|l|}
\hline Doctorando & Clemente Manzanera Reverter \\
\hline Título de la tesis & $\begin{array}{l}\text { Reglas de diseño para la sintonía de controladores predictivos } \\
\text { Dynamic Matrix Control (DMC) }\end{array}$ \\
\hline
\end{tabular}

D. Julio José Ibarrola Lacalle y D. José Manuel Cano Izquierdo, directores de la tesis y D. Clemente Manzanera Reverter, autor de la tesis mencionada, coautores del artículo científico "Tuning rules for a quick start up in Dynamic Matrix Control" publicado en la revista ISA Transactions (índice de impacto 2.6), volume 53 pp 612-627, January 2014.

Exponen:

Que dicho trabajo científico es una contribución relevante de la tesis doctoral presentada. El artículo recoge parte del trabajo producido durante el desarrollo de la tesis, conforme a los objetivos expuestos en el proyecto de tesis. Entre los objetivos cumplidos se encuentran:

- Estudiar el efecto de los parámetros del DMC en la evolución de los polos en lazo cerrado del equivalente LTI.

- Desarrollo de una serie de reglas de sintonía en base al movimiento de los polos en lazo cerrado.

- Validación de las mencionadas reglas mediante simulación y control de de un sistema físico.

- Comparación con otros métodos de ajuste existentes.

Solicitan:

La consideración del artículo presentado como indicio de calidad de la tesis llevada a cabo por D. Clemente Manzanera Reverter, con título "Reglas de diseño para la sintonía de controladores predictivos Dynamic Matrix Control (DMC)".

Y, para que conste, a los efectos que procedan, firman el siguiente documento.

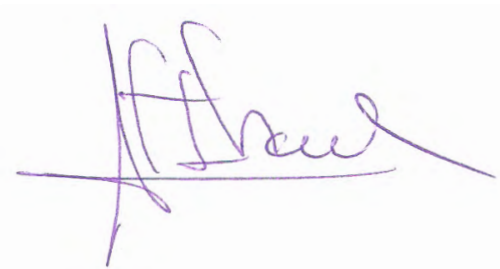

Fdo. Julio José Ibarrola Lacalle

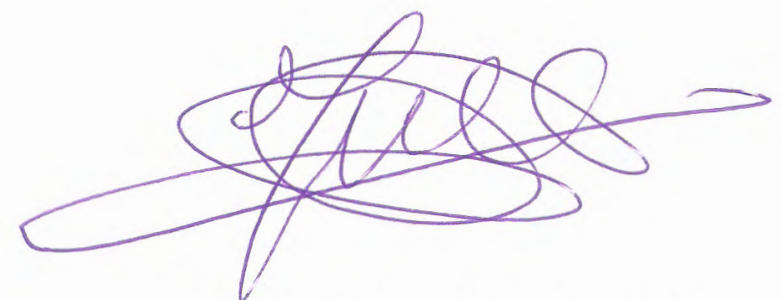

Fdo. José Manuel Cano izquierdo 


\section{ISI Web of Knowledge ${ }^{\text {SM }}$}

\section{Journal Citation Reports ${ }^{\oplus}$}

WELCOME ? HELP

\section{Rank in Category: ISA TRANSACTI ONS}

\section{J ournal Ranking i)}

For 2015, the journal I SA TRANSACTI ONS has an Impact Factor of 2.600.

This table shows the ranking of this journal in its subject categories based on Impact Factor.

\begin{tabular}{|l|c|c|c|}
\hline Category Name & $\begin{array}{c}\text { Total J ournals } \\
\text { in Category }\end{array}$ & $\begin{array}{c}\text { Journal Rank } \\
\text { in Category }\end{array}$ & $\begin{array}{c}\text { Quartile } \\
\text { in Category }\end{array}$ \\
\hline AUTOMATION \& CONTROL SYSTEMS & 59 & 9 & Q1 \\
\hline ENGINEERING, MULTIDISCIPLINARY & 85 & 9 & Q1 \\
\hline INSTRUMENTS \& INSTRUMENTATION & 56 & 6 & Q1 \\
\hline
\end{tabular}

\section{Category Box Plot i}

For 2015, the journal I SA TRANSACTI ONS has an Impact Factor of 2.600.

This is a box plot of the subject category or categories to which the journal has been assigned. It provides information about the distribution of journals based on Impact Factor values. It shows median, 25th and 75th percentiles, and the extreme values of the distribution.

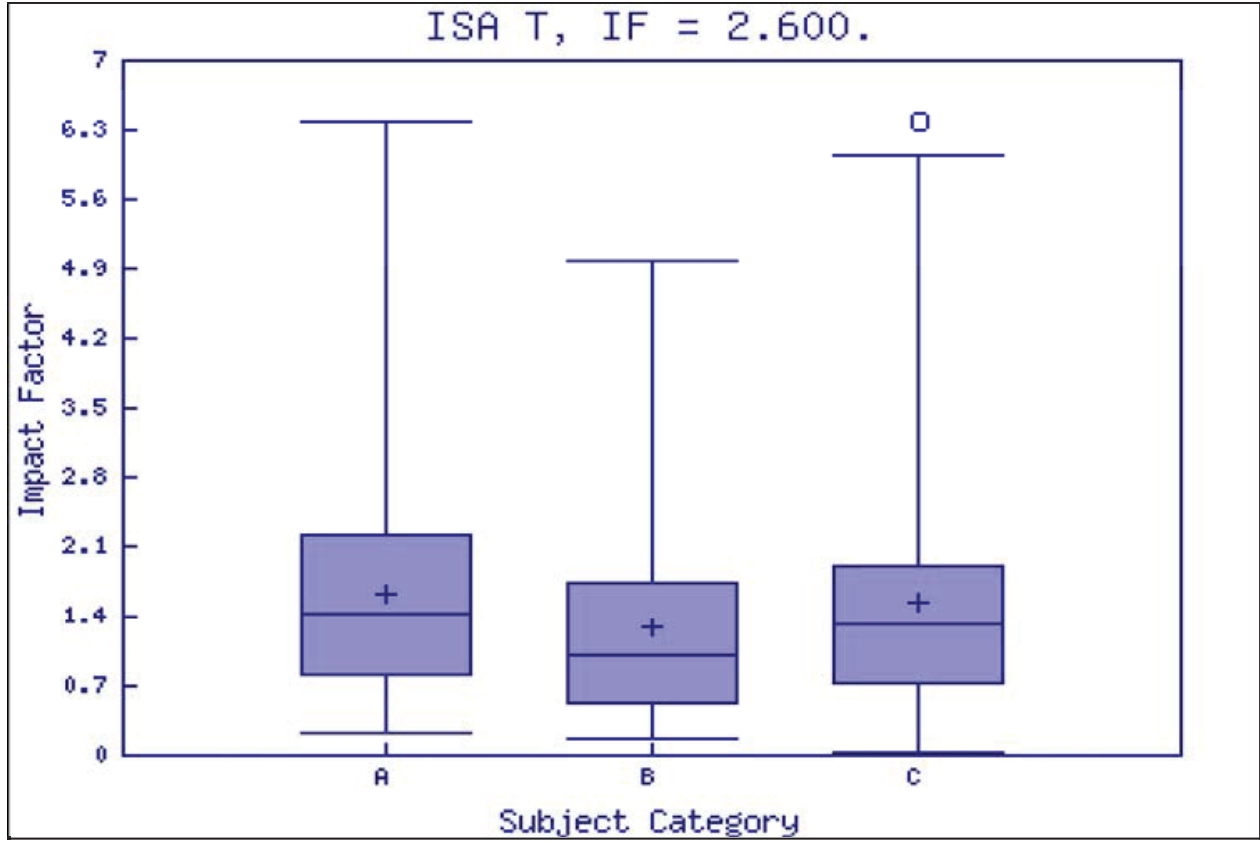

Key

A - AUTOMATION \&

A - CONTROL SYSTEMS

B - ENGINEERING,

B - MULTIDISCIPLINARY

C - INSTRUMENTS \& INSTRUMENTATION 
Research Article

\title{
Tuning rules for a quick start up in Dynamic Matrix Control
}

\author{
Clemente Manzanera Reverter ${ }^{\mathrm{a}, *}$, Julio Ibarrola ${ }^{\mathrm{b}}$, José-Manuel Cano-Izquierdo ${ }^{\mathrm{b}}$ \\ a Polytechnic University of Cartagena, C/Príncipe de Asturias $204^{\circ}$ Derecha, C.P. 30204 Cartagena, Murcia, Spain \\ b Polytechnic University of Cartagena, Universidad Politécnica de Cartagena, Departamento de Sistemas y Automática, \\ Campus Muralla de Mar Calle Doctor Fleming, s/n, C.P. 30202 Cartagena, Murcia, Spain
}

\section{A R T I C L E I N F O}

\section{Article history:}

Received 17 July 2013

Received in revised form

18 November 2013

Accepted 13 December 2013

Available online 23 January 2014

This paper was recommended for

publication by Dr. Rickey Dubay

Keywords:

Dynamic matrix control

Parameters tuning

\begin{abstract}
A B S T R A C T
This paper pretends to offer design rules for the parameters adjustment of the Dynamic Matrix Control (DMC) to allow an easier starting up. The effect on the time response of each algorithm parameter that can be tuned by the user is studied in an unconstrained system. To this aim, the position of the closed loop poles of the equivalent system is calculated. To simplify the study and to obtain more direct conclusions the number of poles will be limited using a First Order Plus Death Time simplification of the real plant. Design rules proposed in this study are tested in some simulated benchmarks and in a real plant.
\end{abstract}

(c) 2013 ISA. Published by Elsevier Ltd. All rights reserved.

\section{Introduction}

Dynamic Matrix Control (DMC) has become a popular Model Predictive Control (MPC) method since it was first introduced by Cutler and Ramaker [12] in the last 70s. It is one of the most used algorithms in industry, but a method for setting its parameters is still being investigated.

There are some mathematical techniques to tune these para meters. A well known algorithm is the one presented by Shridhar and Cooper [1] who introduced a method to calculate the weight ing factor minimizing the condition number of the system matrix. For its calculation the system is approximated by a First Order Plus Dead Time (FOPDT) system. This method is one of the most extended and has been studied by several researchers $[16,14]$. Another example is the algorithm presented by Trierweiler and Farina [2] that uses a Robustness Performance Number (RPN) which indicates how difficult is for a system to reach the required performances with robustness. This method gives directives to calculate the prediction horizon, the control horizon and the sample time. It calculates the system's weighting matrix based on the RPN. This method modifies the normal cost function when it factorizes the system matrix. Han et al. [3] propose a minimiza tion maximization algorithm over a performance index. Garriga and Soroush propose tuning via eigenvalue placement [11].

\footnotetext{
* Corresponding author. Tel.: + 34968 083219; fax: + 34626048569.

E-mail addresses: clmanzanera@hotmail.com (C. Manzanera Reverter), juliojose.ibarrola@upct.es (J. Ibarrola),josem.cano@upct.es (J.-M. Cano-Izquierdo).
}

Some works face a more practical approach using thumb rules given by the experience obtained from simulations and real con trollers. This is a usual approach in industry. The work from Iglesias et al. [4] is an example of this. They present a formula obtained by correlation with data from several simulations. Bagheri and Khaki Sedigh [17] propose an analysis of variance. Wojsznis et al. present the use of heuristic methods [18]. In this category auto tuning methods could be included [8].

Previous works agree on the effect of control horizon and weighting factor but a consensus about what parameter, prediction horizon and weighting factor should be taken as key parameter is not found. Some authors (as Shridhar and Cooper [1]) state that the weighting factor is the key parameter to DMC tuning. But others (as Rossiter [5]) doubt this parameter and defend that the prediction horizon is the factor DMC users should focus in.

Following this goal and trying to make the tuning task easier, this paper pretends to obtain some design rules analysing the effect of changes of DMC parameters on the system closed loop poles (a similar approach to the one used in [11]) in a SISO and unconstrained problem. Time response simulations will be done to evidence the obtained results. These rules will allow users to easily obtain a first set of suitable parameters and help them to predict the effect of a parameter's change on the systems performance. To compute the poles of a DMC controlled system, it will be expressed as a Linear Time Invariant (LTI) (this development can be seen in [6]).

This paper is structured as follows: The first section will be an introduction to the DMC formulation and the DMC expressed as an LTI system. This will allow a better understanding of the following 
section, an analysis of the effect of DMC parameters in closed loop poles and time response from which useful tuning rules will be obtained. The last section will show a validation of the previously mentioned tuning rules by simulation on a benchmark and test on a real system.

\section{DMC algorithm}

As the starting point of this paper is transforming the DMC algorithm in an LTI system, it is mandatory to explain this process. The following paragraphs explain the basis of DMC and how it can be expressed as an LTI system.

\subsection{DMC formulation}

DMC algorithm uses a plant's step response model:

$y(t)=\sum_{i}^{\infty} g_{i} \Delta u(t \quad i)$

where $g_{i}$ are the coefficients of the unit step response, $\Delta u$ is the control increment, $y$ is the system response and $n(t)$ are the disturbances. So predicted values will be (starting predictions from instant $t$ )

$$
\begin{aligned}
\hat{y}(t+k) & =\sum_{i}^{\infty} g_{i} \Delta u(t+k \quad i)+\hat{n}(t+k) \\
& =\sum_{i}^{k} g_{1} \Delta u(t+k \quad i)+\sum_{i}^{\infty} g_{k+1} \Delta u(t+k \quad i)+\hat{n}(t+k)
\end{aligned}
$$

Considering constant disturbances $\left(y_{m}(t)\right.$ being the measured output),

$\hat{n}(t+k)=\hat{n}(t)=y_{m}(t) \quad \hat{y}(t)=y_{m}(t) \quad \sum_{i}^{\infty} g_{i} \Delta u(t \quad i)$

Then Eq. (2) can be written as

$$
\begin{array}{r}
\hat{y}(t+k)=\sum_{i} \sum_{1}^{k} g_{i} \Delta u(t+k \quad i)+\sum_{i}^{\infty} g_{k+1} \Delta u(t+k \quad i)+y_{m}(t) \\
\sum_{i}^{\infty} g_{i} \Delta u(t \quad i)=\sum_{i}^{k} g_{1} \Delta u(t+k \quad i)+f(t+k)
\end{array}
$$

$f(t+k)$ being the free response, the part of the response not depending on future control actions described as follows:

$f(t+k)=y_{m}(t)+\sum_{i}^{\infty}\left(g_{k+i} \quad g_{i}\right) \Delta u(t \quad i)$

If the process is asymptotically stable, coefficients of step response, $g_{i}$, will tend to a constant value after $N$ sample periods, so

$\left(\begin{array}{ll}g_{k+i} & \left.g_{i}\right) \rightarrow 0, \quad i>N\end{array}\right.$

And Eq. (5) can be simplified to

$f(t+k)=y_{m}(t)+\sum_{i}^{N}\left(g_{k+i} g_{i}\right) \Delta u(t \quad i)$

Applying the previous equations for a prediction horizon equal to $\operatorname{Pr}$ and a control horizon equal to $M$ :

$$
\begin{aligned}
& \hat{y}(t+1 / t)=g_{1} \Delta u(t)+f(t+1) \\
& \hat{y}(t+2 / t)=g_{2} \Delta u(t)+g_{1} \Delta u(t+1)+f(t+1) \\
& \vdots \\
& \hat{y}(t+\operatorname{Pr} / t)=\sum_{i} \sum_{\operatorname{Pr}-M+1}^{P r} g_{i} \Delta u(t+\operatorname{Pr} \quad i)+f(t+P r)
\end{aligned}
$$

Defining the system dynamic matrix as

$\mathbf{G}=\left(\begin{array}{cccc}g_{1} & 0 & \ldots & 0 \\ g_{2} & g_{1} & \ldots & 0 \\ g_{M} & g_{M-1} & \ldots & g_{1} \\ : & : & \ldots & : \\ g_{P r} & g_{P r-1} & \ldots & g_{P r-M+1}\end{array}\right)$

Using matricial formulation, it can be written that

$\hat{\mathbf{y}}=\mathbf{G} \boldsymbol{\Delta} \mathbf{u}+\mathbf{f}$

$\hat{\mathbf{y}}$ being a $\operatorname{Pr}$ dimensional vector that contains the future system predictions in the prediction horizon, $\Delta \mathbf{u}$ an $M$ dimensional vector that contains the control increments and $\mathbf{f}$ the free response vector. This expression relates the future outputs with the control incre ments and is used to calculate the necessary action to reach a specific behaviour.

$\mathrm{DMC}^{\prime} \mathrm{s}$ objective is finding a control increment that minimizes a determined cost function that includes errors and control efforts:

$J=\sum_{j}^{p}(\hat{y}(t+j \mid t) \quad w(t+j))^{2}+\sum_{j}^{m} \lambda(\Delta u(t+j \quad 1))^{2}$

$\mathbf{J}=\mathbf{e e}^{\mathrm{T}}+\lambda \Delta \mathbf{u} \Delta \mathbf{u}^{\mathrm{T}}$

where $\mathbf{e}$ is the errors vector and $\Delta \mathbf{u}$ the control efforts vector. In a problem without constraints, the optimized control efforts vector is obtained solving

$\frac{\mathbf{d} \mathbf{J}}{\mathbf{d} \Delta \mathbf{u}}=0$

the result being

$\Delta \mathbf{u}=\left(\mathbf{G}^{\mathbf{T}} \mathbf{G}+\lambda \mathbf{I}\right)^{-\mathbf{1}} \mathbf{G}^{\mathbf{T}}\left(\begin{array}{ll}\mathbf{w} & \mathbf{f}\end{array}\right)$

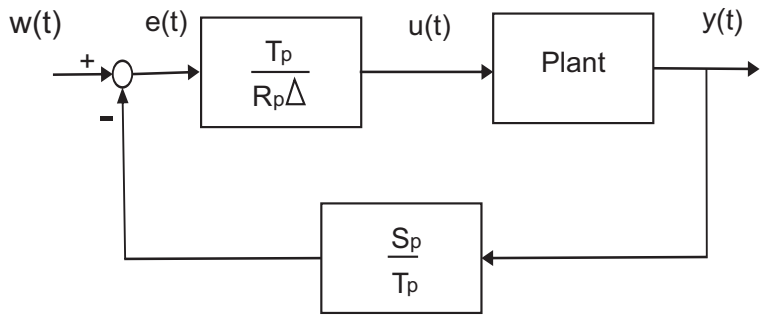

Fig. 1. DMC as an LTI system.

Table 1

Poles for $T 8 \mathrm{~s}$.

\begin{tabular}{llll}
\hline $\operatorname{Pr} 4$ & $\operatorname{Pr} 8$ & $\operatorname{Pr} 12$ & $\operatorname{Pr} 20$ \\
\hline-0.5 & -0.6 & -0.63 & -0.63 \\
$-0.0728+0.5242 \mathrm{i}$ & $-0.1402+0.6233 \mathrm{i}$ & $-0.1747+0.6303 \mathrm{i}$ & $-0.1887+0.6054 \mathrm{i}$ \\
$-0.0728-0.5242 \mathrm{i}$ & $-0.1402-0.6233 \mathrm{i}$ & $-0.1747-0.6303 \mathrm{i}$ & $-0.1887-0.6054 \mathrm{i}$ \\
$0.7814+0.3091 \mathrm{i}$ & $0.6406+0.4251 \mathrm{i}$ & $0.5787+0.4197 \mathrm{i}$ & $0.5275+0.3929 \mathrm{i}$ \\
$0.7814-0.3091 \mathrm{i}$ & $0.6406-0.4251 \mathrm{i}$ & $0.5787-0.4197 \mathrm{i}$ & $0.5275-0.3929 \mathrm{i}$ \\
0.76 & 0.85 & 0.88 & 0.91 \\
\hline
\end{tabular}

Table 2

Poles for $T 16 \mathrm{~s}$.

\begin{tabular}{llll}
\hline $\operatorname{Pr} 2$ & $\operatorname{Pr} 4$ & $\operatorname{Pr} 6$ & $\operatorname{Pr} 10$ \\
\hline-0.29 & -0.45 & -0.52 & -0.55 \\
$0.7418+0.2668 \mathrm{i}$ & $0.4938+0.5175 \mathrm{i}$ & $0.394+0.5588 \mathrm{i}$ & $0.3145+0.5327 \mathrm{i}$ \\
$0.7418-0.2668 \mathrm{i}$ & $0.4938-0.5175 \mathrm{i}$ & $0.394-0.5588 \mathrm{i}$ & $0.3145-0.5327 \mathrm{i}$ \\
0.49 & 0.77 & 0.81 & 0.85 \\
\hline
\end{tabular}




\subsection{Parameters summary}

The following parameters can be deduced from the previous section:

- Prediction horizon $(\mathrm{Pr})$ : Indicates the time horizon during which the output must follow the setpoint. It also may be expressed as the period comprised between $N_{1}$ and $N_{2}$.
- Control horizon $(M)$ : Indicates the future time steps for which control increments are calculated.

- Sample time $(T)$.

- Model horizon $\left(n_{g}\right)$ : Indicates the time interval from the step response that is used as model.

- Weighting factor $(\lambda)$ : This coefficient penalizes the control signal to avoid it to be too aggressive and the response oscillating. It also has the effect of giving mathematical robustness to the

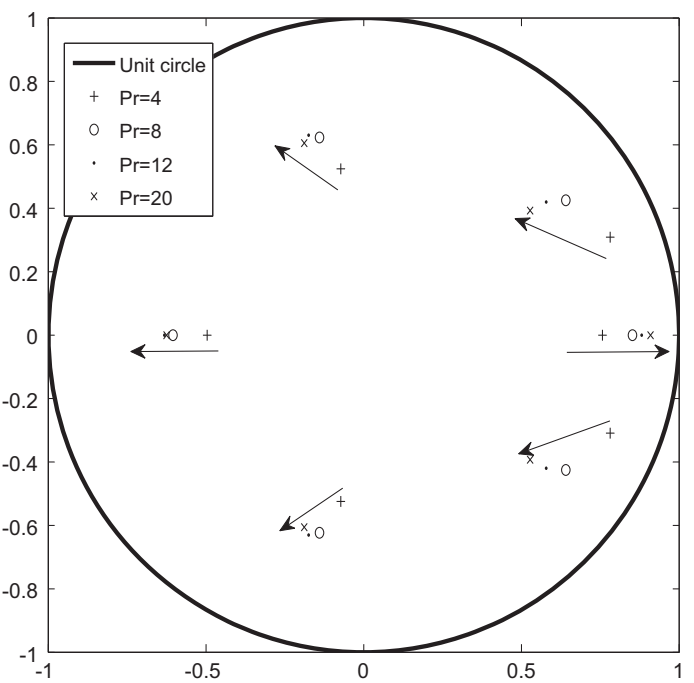

Closed loop Poles for $\mathrm{T} 8$ seconds

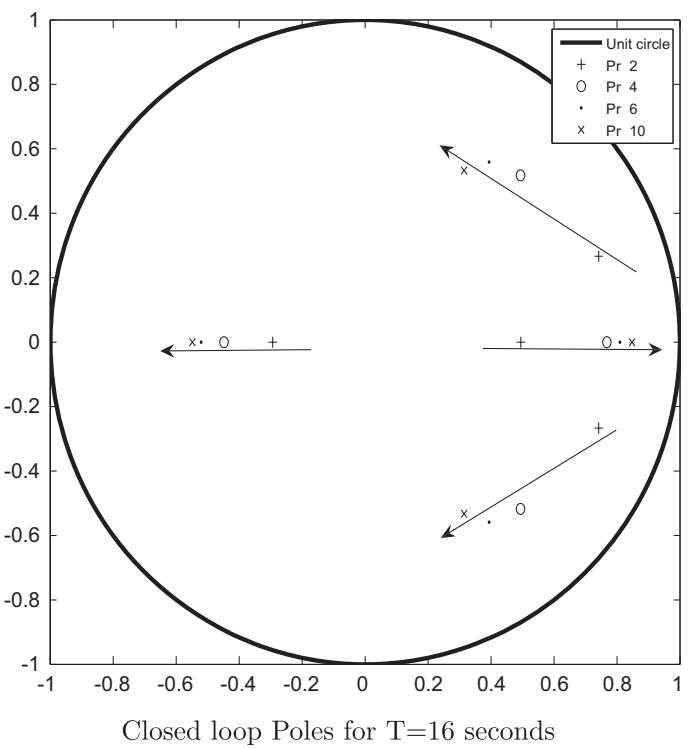

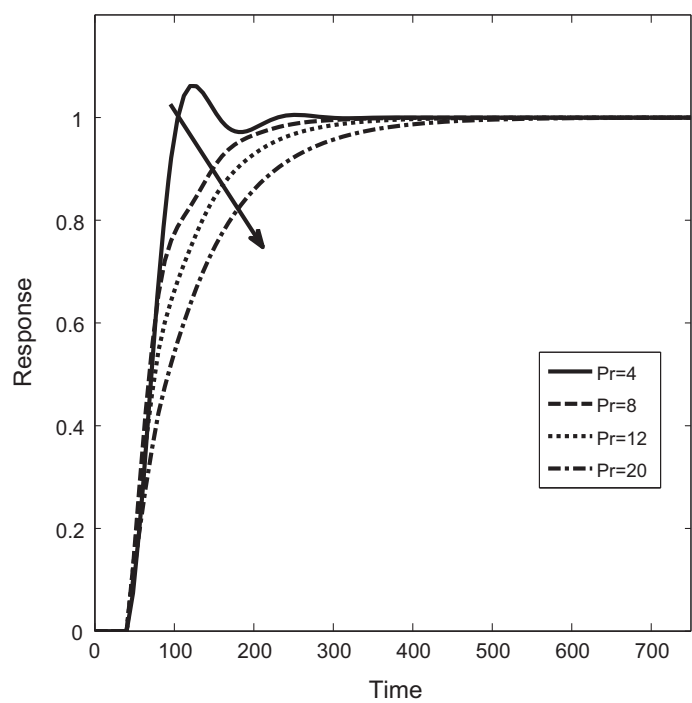

System response for $\mathrm{T} 8$ seconds and different values of $\operatorname{Pr}$

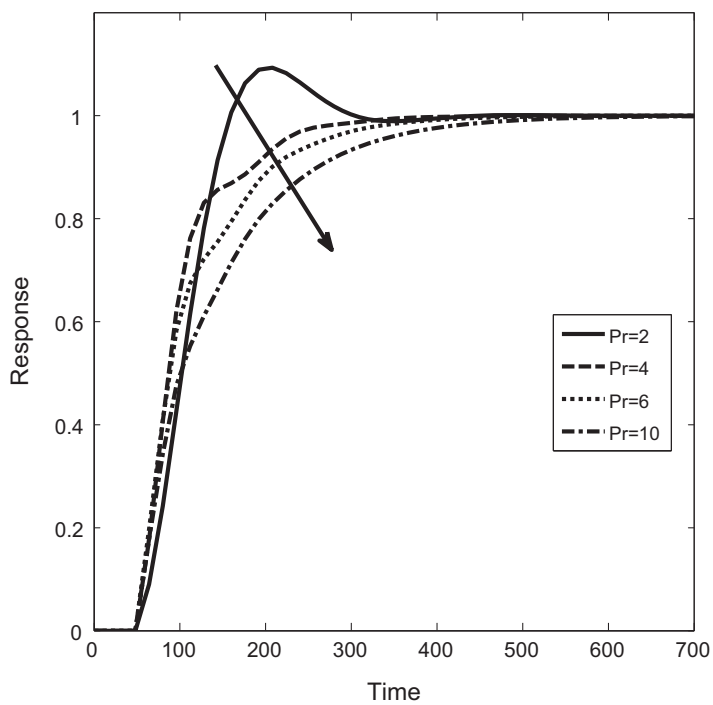

System response for $\mathrm{T}=16$ seconds and different values of $\mathrm{Pr}$

Fig. 2. Poles location and time response for $T 8 \mathrm{~s}$ and $T 16 \mathrm{~s}$.

Table 3

Poles for different $M$ values.

\begin{tabular}{llllr}
\hline \multicolumn{1}{l}{1} & $M 2$ & $M \quad 3$ & $M \quad 4$ & \multicolumn{1}{l}{5} \\
\hline 0.7680 & 0.7536 & 0.7482 & 0.7475 & 0.7475 \\
$0.4938+0.5175 \mathrm{i}$ & $0.5293+0.4624 \mathrm{i}$ & $0.5385+0.4505 \mathrm{i}$ & $0.5396+0.4494 \mathrm{i}$ & $0.5396+0.4494 \mathrm{i}$ \\
$0.4938-0.5175 \mathrm{i}$ & $0.5293-0.4624 \mathrm{i}$ & $0.5385-0.4505 \mathrm{i}$ & $0.5396-0.4494 \mathrm{i}$ & $0.5396-0.4494 \mathrm{i}$ \\
-0.4480 & -0.4049 & -0.3966 & -0.3958 & -0.3958 \\
\hline
\end{tabular}


method minimizing the effect of having a $\left(\mathbf{G}^{\mathbf{T}} \mathbf{G}+\boldsymbol{\lambda} \mathbf{I}\right)$ matrix not possible to invert.

\subsection{DMC expressed as LTI model}

In order to apply system analysis methods to the DMC, it can be expressed as an LTI model. The output prediction will be

$\hat{y}(t+k)=G \Delta u(t+k)+f(t+k)$

The first component of the optimal controls vector will be

$\Delta u_{\text {opt }}=\mathbf{K e}$

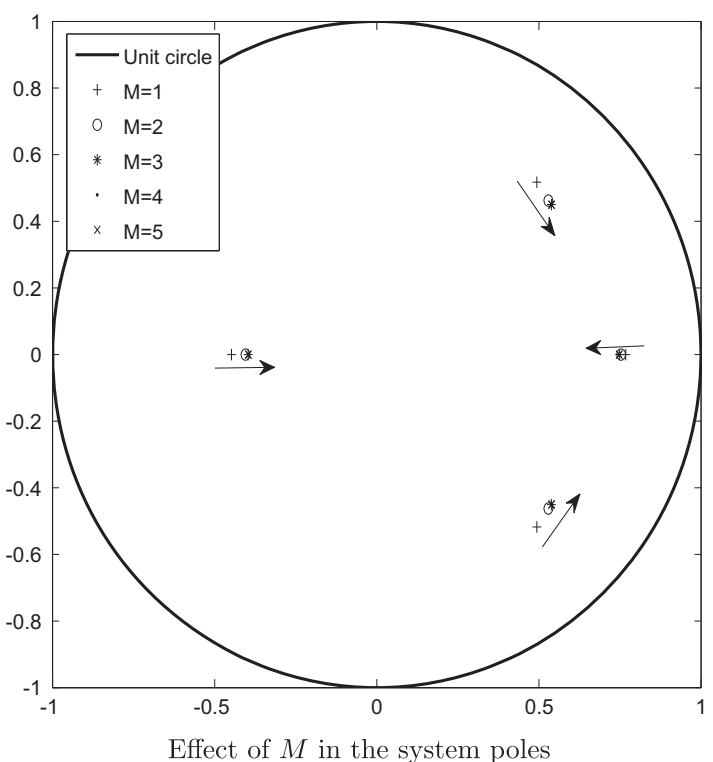

Fig. 3. Poles location and time response for different values of $M$.

Table 4

Poles for different M values and $\operatorname{Pr} 10$.

\begin{tabular}{llllr}
\hline \multicolumn{1}{l}{1} & $M \quad 2$ & $M \quad 3$ & $M \quad 4$ & 5 \\
\hline 0.8478 & 0.8111 & 0.7830 & 0.7723 & 0.7698 \\
$0.3084+0.5373 \mathrm{i}$ & $0.4401+0.4739 \mathrm{i}$ & $0.4856+0.4722 \mathrm{i}$ & $0.4985+0.4812 \mathrm{i}$ & $0.5009+0.4856 \mathrm{i}$ \\
$0.3084-0.5373 \mathrm{i}$ & $0.4401-0.4739 \mathrm{i}$ & $0.4856-0.4722 \mathrm{i}$ & $0.4985-0.4812 \mathrm{i}$ & $0.5009-0.4856 \mathrm{i}$ \\
-0.5578 & -0.4279 & -0.4177 & -0.4217 & -0.4242 \\
\hline
\end{tabular}

Table 5

Poles for different $M$ values and $\operatorname{Pr} 60$

\begin{tabular}{|c|c|c|c|c|}
\hline$M \quad 1$ & $M 2$ & $M 3$ & $M 4$ & $M 5$ \\
\hline 0.9075 & 0.8105 & 0.7687 & 0.7650 & 0.7683 \\
\hline $0.2368+0.4291 \mathrm{i}$ & $0.4388+0.4788 \mathrm{i}$ & $0.4859+0.5369 \mathrm{i}$ & $0.4886+0.5474 \mathrm{i}$ & $0.4882+0.5323$ \\
\hline $0.2368-0.4291 \mathrm{i}$ & $0.4388-0.4788 \mathrm{i}$ & $0.4859-0.5369 \mathrm{i}$ & $0.4886-0.5474 \mathrm{i}$ & $0.4882-0.5323$ \\
\hline-0.4822 & -0.4321 & -0.4642 & -0.4712 & -0.4601 \\
\hline
\end{tabular}

Table 6

Poles for different $M$ values and $\operatorname{Pr} 120$.

\begin{tabular}{llllr}
\hline \multicolumn{1}{l}{1} & $M \quad 2$ & $M \quad 3$ & $M \quad 4$ & 5 \\
\hline 0.9119 & 0.8084 & 0.7673 & 0.7646 & 0.7684 \\
$0.2320+0.4202 \mathrm{i}$ & $0.4399+0.4842 \mathrm{i}$ & $0.4851+0.5456 \mathrm{i}$ & $0.4869+0.5536 \mathrm{i}$ & $0.4868+0.5356 \mathrm{i}$ \\
$0.2320-0.4202 \mathrm{i}$ & $0.4399-0.4842 \mathrm{i}$ & $0.4851-0.5456 \mathrm{i}$ & $0.4869-0.5536 \mathrm{i}$ & $0.4868-0.5356 \mathrm{i}$ \\
-0.4737 & -0.4362 & -0.4709 & -0.4763 & -0.4630 \\
\hline
\end{tabular}




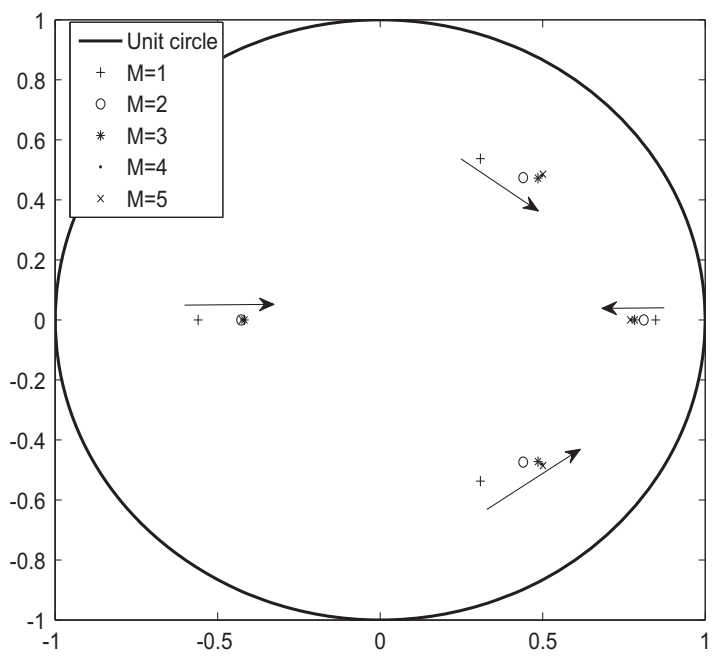

Effect of $M$ in the system poles and $\operatorname{Pr}=10$

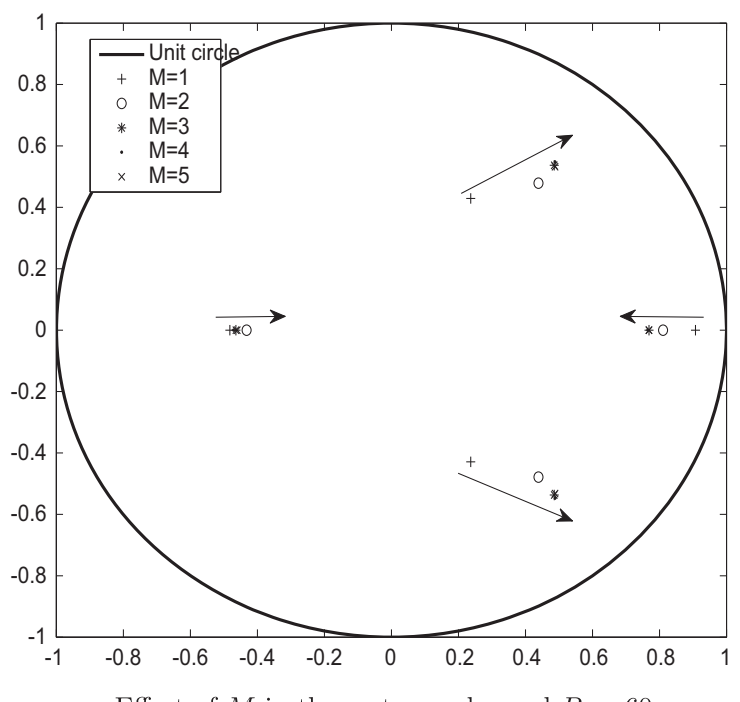

Effect of $M$ in the system poles and $\operatorname{Pr}=60$

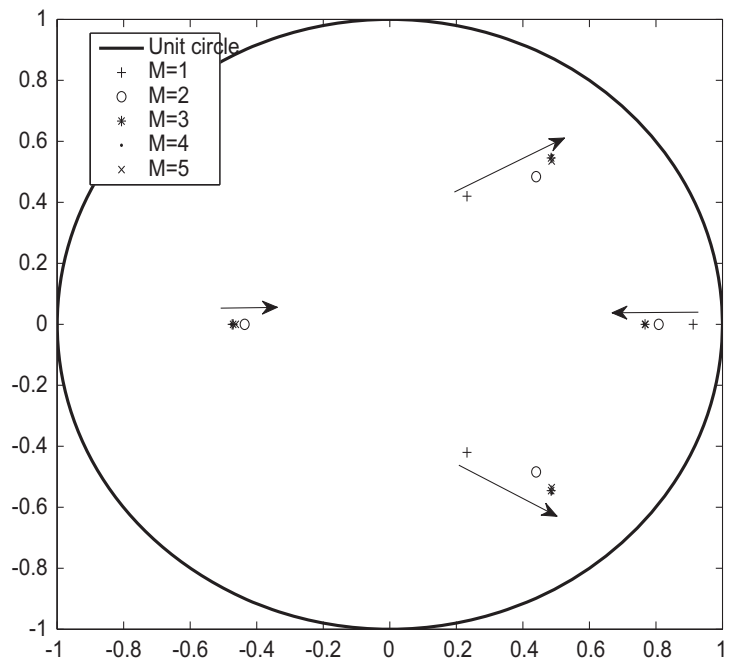

Effect of $M$ in the system poles and $\operatorname{Pr}=120$

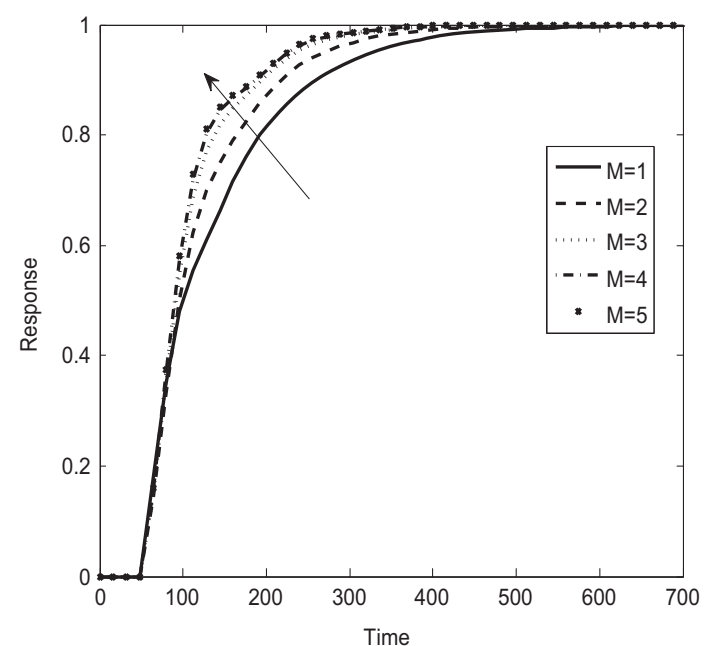

System response for different $M$ values and $\operatorname{Pr}=10$

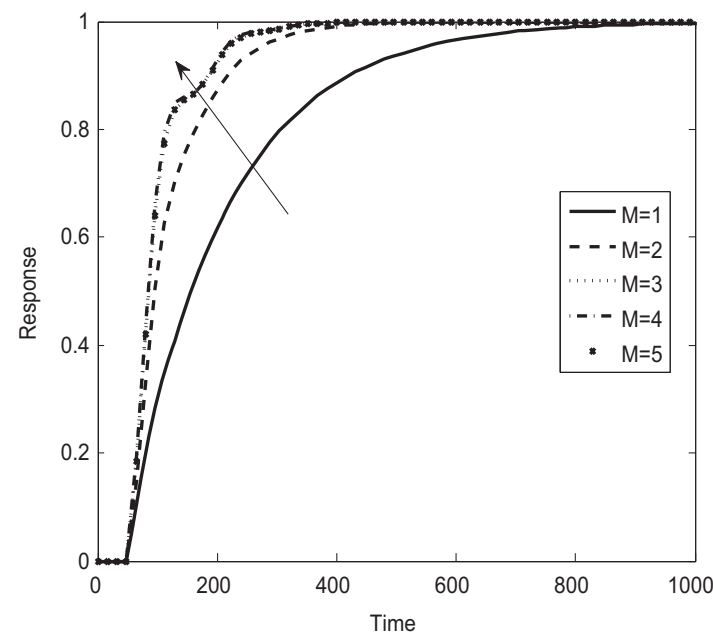

Response for different $M$ values and $\mathrm{Pr}=60$

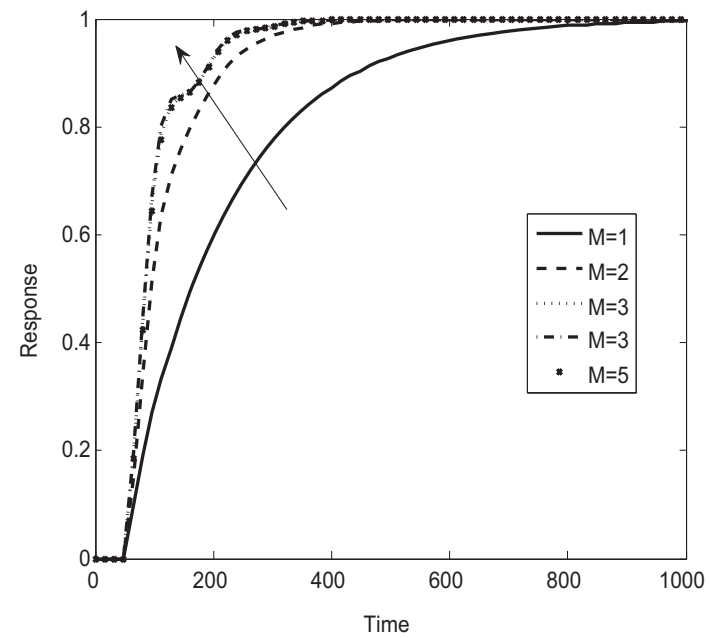

Response for different $M$ values and $\operatorname{Pr}=120$

Fig. 4. Poles location and time response varying $\operatorname{Pr}$ and $M$. 


$$
\Delta u_{o p t}(t)=\sum_{t}^{P r} k_{i} w(t+i) \quad \sum_{t}^{P r} k_{i} y(t) \quad \sum_{t}^{P r} k_{1} S_{n}^{i} \Delta u(t)
$$

Comparing this expression with a two degree of freedom con troller (see Fig. 1),

$R_{p}\left(q^{-1}\right) \Delta u(t)=T_{p}\left(q^{-1}\right) w(t) \quad S_{p}\left(q^{-1}\right) y(t)$

The DMC can be expressed as a block diagram as shown in Fig. 1, where

$R_{P}=1+\sum_{i}^{P r} k_{1} S_{n}^{i}$

$T_{P}=\sum_{i}^{P r} k_{i} q^{i}$

$S_{P}=\sum_{i}^{P r} k_{i}$

\section{Effect of DMC parameters in system response}

To study the effect of prediction horizon $(P r)$, sample time $(T)$, weighting factor $(\lambda)$, control horizon $(M)$ and model Horizon $\left(n_{g}\right)$ in the time response, an analysis of the closed loop poles will be made. As already explained in Section 2.3 a benchmark controlled with a DMC algorithm can be expressed as an LTI system whose poles can be calculated. Those poles will depend on the values of the DMC parameters and the step response coefficients. If the behaviour of the poles when the DMC parameters change can be deduced, we will be able to understand their influence in the response to a unit step. If the influence in the time response is known, then some useful heuristic design rules (with the same philosophy than the Ziegler Nichols method for PID controllers [13]) can be developed. The first process used in [1], taking a time delay of $16 \mathrm{~s}$, has been chosen to compute the poles location movements with parameter changes:

$G(s)=\frac{e^{-16 s}}{(150 s+1)(24 s+1)}$

The conclusions taken from the study of the benchmark for Eq. (28) must be applicable to many systems. The study will not be made on the benchmark "as it", but to its First Order Plus Dead Time (FOPDT) equivalent (Eq. (29)). This ensures that the results of the study will be applicable to other first order systems and, consequently, to any system that can be simplified with a FOPDT:

$G(s)=\frac{e^{-32 s}}{157 s+1}$

The analysis will be done at $T=8 \mathrm{~s}$ and $T=16 \mathrm{~s}$. These sample times have been selected to match with the time delay of $32 \mathrm{~s}$ of the FOPDT and as close as possible to $0.05 \tau$ and $0.1 \tau$ as performed by Shridhar and Cooper [1] ( $\tau$ is the time constant of the corresponding FOPDT). Eqs. (30) and (31) are the discrete form of Eq. (29) for a sample time of 8 and $16 \mathrm{~s}$ respectively:

$$
\begin{aligned}
& G_{8}(z)=\frac{0.04968 z^{-5}}{10.9503 z^{-1}} \\
& G_{16}(z)=\frac{0.09516 z^{-3}}{1 \quad 0.9048 z^{-1}}
\end{aligned}
$$

\subsection{Effect of prediction horizon and sample time in the process}

As stated before, this analysis will be done at $T=8 \mathrm{~s}$ and $T=16 \mathrm{~s}$. Tables 1 and 2 show the obtained poles for these sample times. Fig. 2 allows to observe the evolution of system poles when
$T$ and $\operatorname{Pr}$ change. In Fig. 2 each pole has a symbol (indicated in Tables 1 and 2) depending on its Pr.

The prediction horizon has been selected to make prediction time $(\mathrm{Pr} \cdot \mathrm{T})$ nearly the same for both sample times and have comparable results. The first conclusion it can be reached from Tables 1 and 2 and Fig. 2 is that increasing prediction horizon increases the value of real poles and decreases the module of complex poles. This makes the real positive poles become the dominant ones and dictates the behaviour of the system. The real positive poles produce an oscillation free time response. So if a time response free of oscillations is required, increasing $\operatorname{Pr}$ seems to be the correct choice. It can also be concluded that as the prediction horizon grows, its effect on the poles becomes weaker and the system response approaches to an open loop system. $\operatorname{Pr}$ has a maximum useful value and increasing it beyond this value will not vary system response, but it will make the system slower.

Fig. 2 shows that as Pr is increased the system's response is slower (it approaches to an open loop system). As the dominant pole approaches the unit circle, the response time of the system grows. In order to have an oscillation free and not too slow response Pr must be carefully chosen. The key is using a value that makes the real positive pole clearly dominant. For $T=16 \mathrm{~s}$ this value would be $P r=6$ as the complex poles have a module of 0.68 versus 0.81 of the dominant pole. For $T=8 \mathrm{~s}$, it would be $P r=8$ (module of the dominant pole is 0.85 versus 0.618 of the complex pole).

Tables 1 and 2 show that the lower the sample time is, the closer the poles to the unit circle are. Another effect is that the poles are much closer among them. This makes that for small prediction horizons complex poles are not so dominant, so the response improves with respect to the same prediction time but higher sample time. But as the prediction horizon is increased and the real positive poles become dominant, the difference between the poles calculated for each sample times is difficult to appreciate.

\begin{tabular}{|c|c|c|c|}
\hline $\operatorname{Pr} 2$ & $\operatorname{Pr} 4$ & $\operatorname{Pr} 6$ & $\operatorname{Pr} 10$ \\
\hline \multicolumn{4}{|l|}{$\lambda 0$} \\
\hline-0.97 & -0.78 & -0.7 & -0.61 \\
\hline $0.2983+0.8792 \mathrm{i}$ & $0.3089+0.7199 \mathrm{i}$ & $0.3001+0.6382 \mathrm{i}$ & $0.2815+0.5552 \mathrm{i}$ \\
\hline $0.2983-0.8792 \mathrm{i}$ & $0.3089-0.7199 \mathrm{i}$ & $0.3001-0.6382 \mathrm{i}$ & $0.2815-0.5552 \mathrm{i}$ \\
\hline 0.75 & 0.79 & 0.81 & 0.85 \\
\hline \multicolumn{4}{|l|}{$\lambda \quad 0.25$} \\
\hline-0.29 & -0.45 & -0.52 & -0.55 \\
\hline $0.7418+0.2668 \mathrm{i}$ & $0.4938+0.5175 i$ & $0.394+0.5588 \mathrm{i}$ & $0.3145+0.5327 i$ \\
\hline $0.7418-0.2668 \mathrm{i}$ & $0.4938-0.5175 \mathrm{i}$ & $0.394-0.5588 \mathrm{i}$ & $0.3145-0.5327 i$ \\
\hline 0.49 & 0.77 & 0.81 & 0.85 \\
\hline 0 & 0 & 0 & 0 \\
\hline \multicolumn{4}{|l|}{$\lambda \quad 0.50$} \\
\hline-0.22 & -0.35 & -0.43 & -0.5 \\
\hline $0.854+0.1877 i$ & $0.5646+0.3613 \mathrm{i}$ & $0.4494+0.4828 \mathrm{i}$ & $0.342+0.5091 \mathrm{i}$ \\
\hline $0.854-0.1877 \mathrm{i}$ & $0.5646-0.3613 \mathrm{i}$ & $0.4494-0.4828 \mathrm{i}$ & $0.342-0.5091 \mathrm{i}$ \\
\hline 0.29 & 0.73 & 0.8 & 0.85 \\
\hline 0 & 0 & 0 & 0 \\
\hline \multicolumn{4}{|l|}{$\lambda \quad 0.75$} \\
\hline-0.18 & -0.3 & -0.38 & -0.46 \\
\hline $0.8876+0.1544 \mathrm{i}$ & $0.6435+0.2185 \mathrm{i}$ & $0.4859+0.4139 i$ & $0.3651+0.4851 \mathrm{i}$ \\
\hline $0.8876-0.1544 \mathrm{i}$ & $0.6435-0.2185 \mathrm{i}$ & $0.4859-0.4139 i$ & $0.3651-0.4851 \mathrm{i}$ \\
\hline 0.23 & 0.62 & 0.8 & 0.85 \\
\hline 0 & 0 & 0 & 0 \\
\hline \multicolumn{4}{|l|}{$\lambda 1$} \\
\hline-0.16 & -0.26 & -0.34 & -0.43 \\
\hline $0.904+0.1334 \mathrm{i}$ & $0.7524+0.1818 \mathrm{i}$ & $0.5129+0.3498 \mathrm{i}$ & $0.3847+0.4614 i$ \\
\hline $0.904-0.1334 \mathrm{i}$ & $0.7524-0.1818 \mathrm{i}$ & $0.5129-0.3498 \mathrm{i}$ & $0.3847-0.4614 i$ \\
\hline 0.19 & 0.43 & 0.79 & 0.84 \\
\hline 0 & 0 & 0 & 0 \\
\hline
\end{tabular}

Table 7

Poles evolution with $\lambda$. 


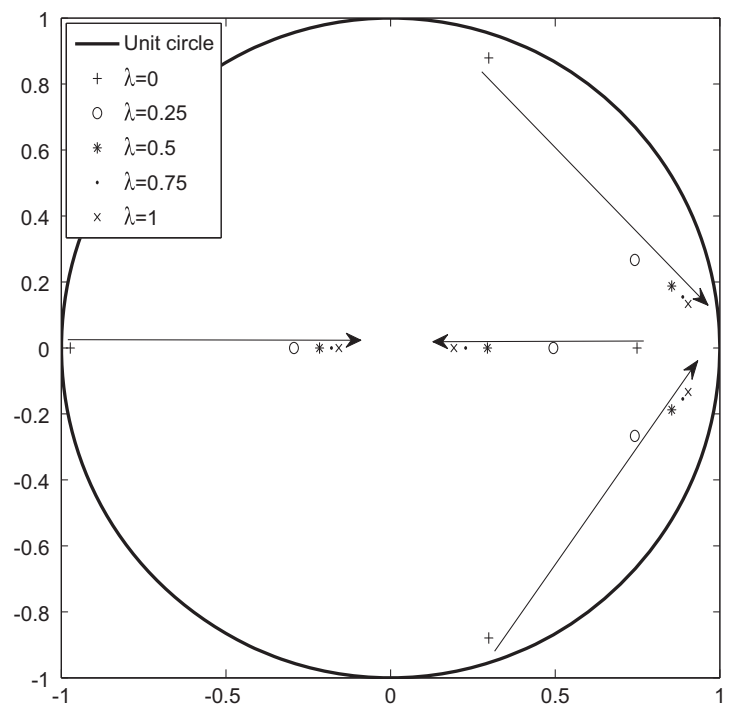

Poles evolution with $\lambda(\operatorname{Pr}=2)$

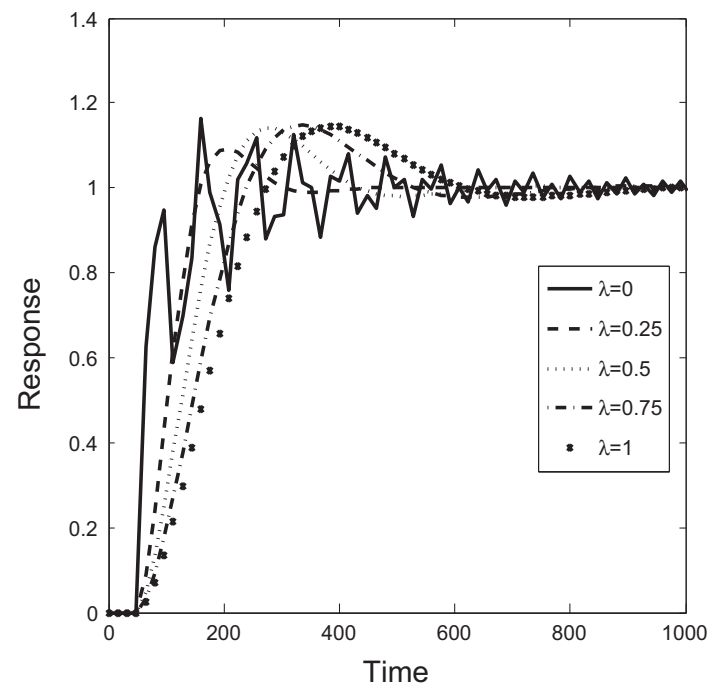

System response for different $\lambda$ values

Fig. 5. Poles location and time response for different values of $\lambda$.

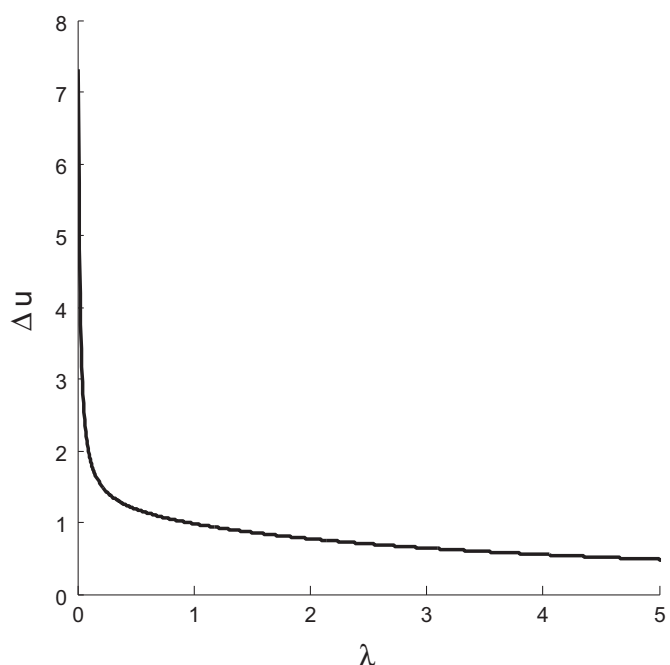

Fig. 6. Control increment-weighting factor curve.

\subsection{Effect of control horizon in the process}

Works by previous researchers (for example Shridhar and Cooper [1]) show that this parameter has a small influence in the process. Various simulations are done varying the control horizon while the other parameters $(\lambda=0.25)$ are kept constant. Obtained poles for process of Eq. (31) are shown in Table 3. This table also shows the symbol used for each value of $\lambda$ in Fig. 3 .

As it can be seen in Fig. 3, poles (and consequently, response) vary very slightly when $M$ changes. It may seem that $M$ has a small influence in the process as pole's location shows a minimum change when this parameter changes. This is because the influence of $M$ depends on $\operatorname{Pr}$ and $\lambda$. Let us see what happens when $\operatorname{Pr}$ is increased from $\operatorname{Pr}=4$ up to different values (Tables 46 and Fig. 4). As it can be seen with $P r$ higher than the FOPDT time constant, the effect of $M$ becomes more important reducing notably the domi nant pole's value and, consequently, the settling time. However it must be paid attention due to the fact that complex poles imaginary and real components are being increased too and this may deteriorate the response if those poles become dominant.

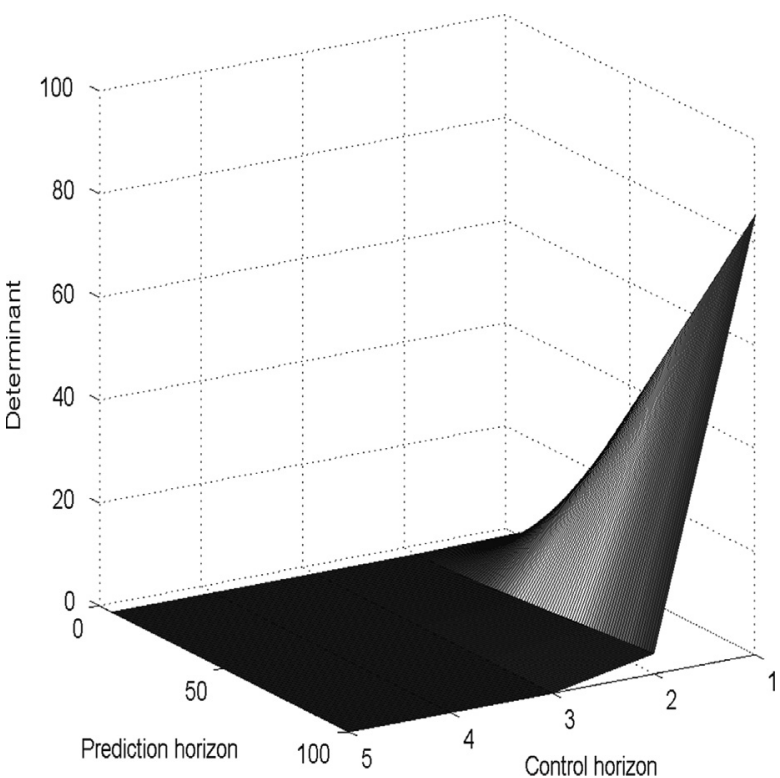

Fig. 7. $G^{T} G$ determinant for $\lambda \quad 0$.

When a certain value of $M$ is reached the effect on poles' location gets weaker and only little changes are observed when this parameter increases.

\subsection{Effect of weighting factor}

The weighting factor has the effect of softening the system response, but it does in an opposite way than the prediction horizon. Table 7 and Fig. 5 (the arrow points on the direction of increasing $\lambda$ ) show that as $\lambda$ increases the real positive pole and the imaginary part of the complex poles decrease while the complex pole real part grows. This makes the dominant pole a complex one and the system response presents peaks and oscilla tions, as it can be seen in Fig. 5. The effect of softening the response is explained by the fact that as the real part of the pole is increased and the imaginary part decreased the pole approaches to a real pole and its corresponding response. When the module of 
the pole is increased, the settling time also grows. This parameter's effect is conditioned by the prediction horizon. Its effect becomes very difficult to appreciate as $\operatorname{Pr}$ and $\lambda$ grow. Its effect arises when it becomes higher than zero (see Table 7) and weakens as it becomes higher.

The effects of $\lambda$ become clear, but we still need to find the correct value for it. As it has been seen in Table 7 and Fig. 5, the effect of $\lambda$ becomes negligible when a certain value has been reached. It must be pointed that the DMC solution (Eq. (16)) could be summarized as the product of a matrix, $\mathbf{K}$, independent of time

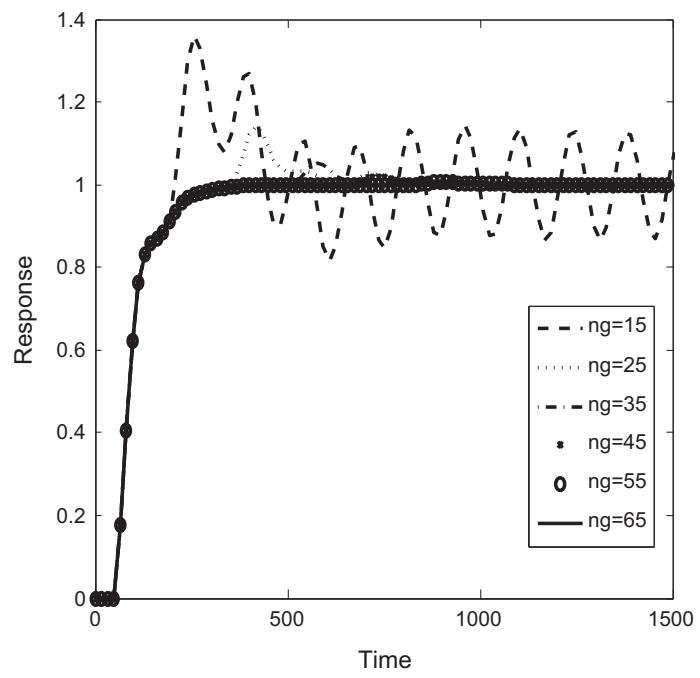

System response varying $n_{g}$

Fig. 8. Time response for different values of $n_{g}$.

Table 8

Poles for $\lambda \quad 0$ and $\operatorname{Pr} 100$.

\begin{tabular}{llll}
\hline$M 1$ & $M 2$ & $M 3$ & $M$ \\
\hline-0.4764 & -1.2207 & -1.2207 & -1.2207 \\
$0.2323+0.4221 \mathrm{i}$ & $0.2481+1.0340 \mathrm{i}$ & $0.2481+1.0340 \mathrm{i}$ & $0.2481+1.0340 \mathrm{i}$ \\
$0.2323-0.4221 \mathrm{i}$ & $0.2481-1.0340 \mathrm{i}$ & $0.2481-1.0340 \mathrm{i}$ & $0.2481-1.0340 \mathrm{i}$ \\
0.9111 & 0.7245 & 0.7245 & 0.7245 \\
\hline
\end{tabular}

and the vector of errors, e, which changes at every time step (Eq. (32)). In the first time step the value of the control increment can be calculated without the need of running a simulation or process. It just must be taken into account that the free response, $\mathbf{f}$, in this first time step is zero. This would make the errors vector equal to the setpoint, which is a known datum:

$\Delta \mathbf{u}=\left(\mathbf{G}^{\mathbf{T}} \mathbf{G}+\lambda \mathbf{I}\right)^{-1} \mathbf{G}^{\mathbf{T}}\left(\begin{array}{ll}\mathbf{w} & \mathbf{f}\end{array}\right)=\mathbf{K}(\mathbf{W} \quad \mathbf{f})=\mathbf{K e}$

This value of first control increment can be calculated for several values of $\lambda$ and a curve can be built. For very small values of $\lambda$ the first control increment rises to very high values, which points out to an unstable response. For big values of $\lambda$, the value of the control increment decreases very slowly (this is in accordance with previous results). There is a range of values of $\lambda$ in which the control increment changes significantly. Weighting factor should be within that range.

In Fig. 6 the relationship between the weighting factor and the first control increment for two fixed values of prediction and control horizon is shown. It can be seen that, as expected, the value of the first control increment decreases when $\lambda$ becomes higher. It also arrives to a point when further increase of $\lambda$ has a little impact on the control increment.

The weighting factor is also closely related to the mathematical robustness of the algorithm. As it can be seen in Eq. (16), if $\lambda$ takes a value of 0 the DMC result would depend on $G^{T} G$ inverse matrix. In case $G^{T} G$ could not be inversed (its determinant is zero) or close to zero, the system would become unstable. Fig. 7 shows the evolution of this determinant with $M$ and $\operatorname{Pr}$ for a fixed value of $\lambda$. It can be seen that, when the value of $\lambda$ is too low (e.g zero for this graph), as $M$ grows the value of the determinant of $G^{T} G$ decreases and could eventually become zero, making impossible to calculate any control increment.

\subsection{Effect of model horizon}

This parameter gives the step response time which is used as model, $n_{g}$ is the number of step response coefficients $(g)$ taken. The response will improve increasing $n_{g}$ up to a point when it will not be affected by it. Model horizon is involved in the calculation of free response in the DMC algorithm, Eq. (5). When all $g_{i+k}$ coefficients become equal to $g_{i}$ this term will become zero. All additional $g$ coefficients that increase $n_{g}$ beyond this limit do not
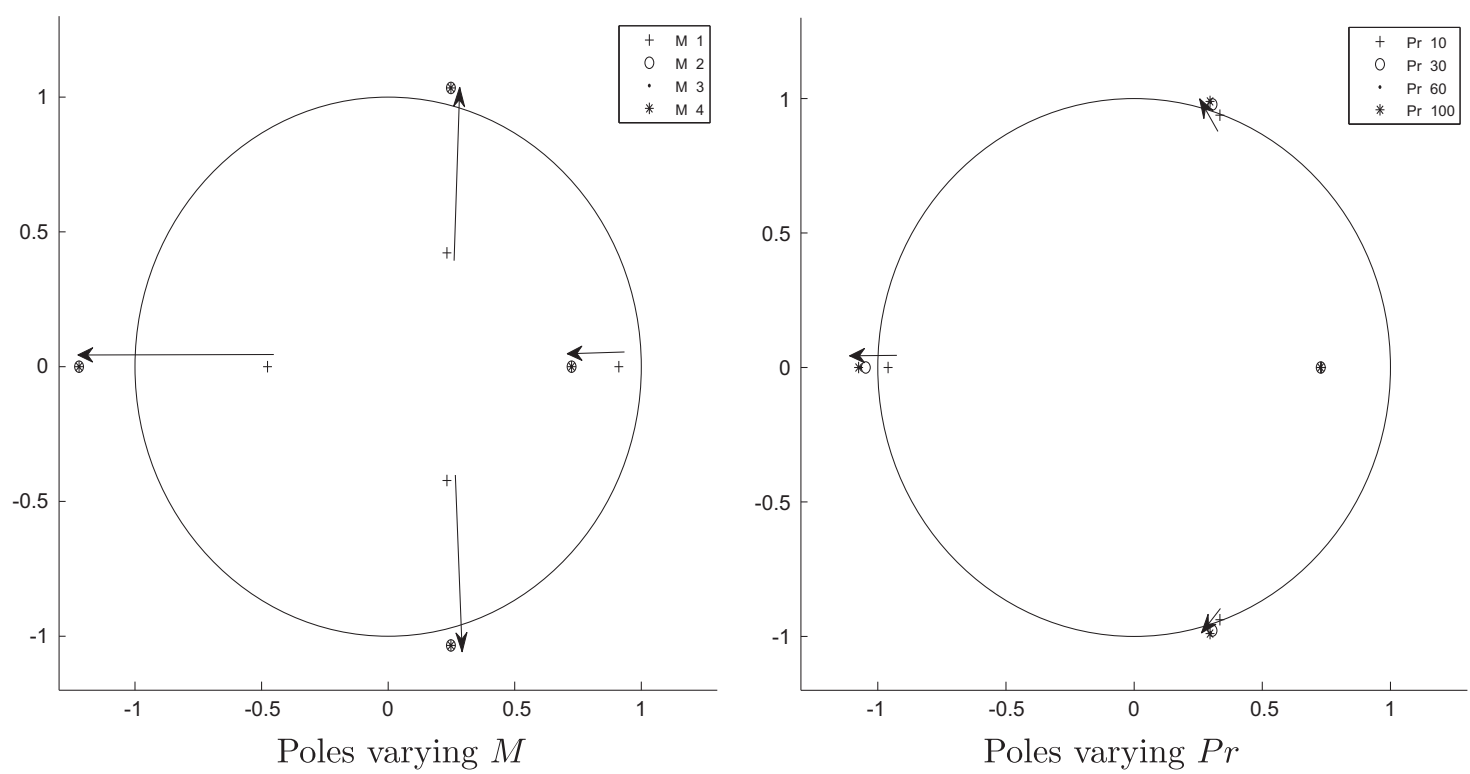

Fig. 9. Poles for $\lambda \quad 0 \operatorname{Pr} 100$ and $\lambda \quad 0.005$ and $M 2$. 
have any effect. DMC is applied to stable processes, where there is a value of $n_{g}$ from which $g_{k} \quad g_{k-1}=0$. If this value is the settling time $(n)$, then $n_{g}=n+\operatorname{Pr}$. So $n_{g}$ depends on the prediction horizon and the sample time. For the process being used as an example (Eq. (29)) settling time is $950 \mathrm{~s}$ approximately. For sample time $16 \mathrm{~s}$ this means $n=60$. For $\operatorname{Pr}=4$, the ideal $n_{g}$ would be 84 . Fig. 8 shows the evolution of the system response from $n_{g}=15$ to $n_{g}=65$ for the DMC. As it can be seen only small values of $n_{g}$ give a poor performance. From $n_{g}>1 / 2 n$ the output prediction is accurate enough.

Table 9

Poles for $\lambda \quad 0.005$ and $M 2$.

\begin{tabular}{llll}
\hline $\operatorname{Pr} 10$ & $\operatorname{Pr} 30$ & $\operatorname{Pr} 60$ & $\operatorname{Pr} 100$ \\
\hline-0.9597 & -1.0467 & -1.0683 & -1.0746 \\
$0.3345+0.9379 \mathrm{i}$ & $0.3057+0.9783 \mathrm{i}$ & $0.2984+0.9868 \mathrm{i}$ & $0.2963+0.9892 \mathrm{i}$ \\
$0.3345-0.9379 \mathrm{i}$ & $0.3057-0.9783 \mathrm{i}$ & $0.2984-0.9868 \mathrm{i}$ & $0.2963-0.9892 \mathrm{i}$ \\
0.7314 & 0.7282 & 0.7275 & 0.7274 \\
\hline
\end{tabular}

Table 10

Poles for $\lambda \quad 0.075, \operatorname{Pr} 100$ varying $M$

\begin{tabular}{|c|c|c|c|}
\hline$M \quad 1$ & $M 2$ & M 3 & M 4 \\
\hline-0.4760 & -0.5807 & -0.6109 & -0.5818 \\
\hline $0.2325+0.4221 \mathrm{i}$ & $0.4412+0.6557 i$ & $0.4480+0.6961 i$ & $0.4512+0.6643 i$ \\
\hline $0.2325-0.4221 \mathrm{i}$ & $0.4412-0.6557 \mathrm{i}$ & $0.4480-0.6961 \mathrm{i}$ & $0.4512-0.6643 \mathrm{i}$ \\
\hline 0.9111 & 0.7638 & 0.7523 & 0.7579 \\
\hline
\end{tabular}

Table 11

Poles for $\lambda \quad 0.075, M \quad 2$ varying $P r$

\begin{tabular}{llll}
\hline $\operatorname{Pr} 10$ & $\operatorname{Pr} 30$ & $\operatorname{Pr} 60$ & $\operatorname{Pr} 100$ \\
\hline-0.5104 & -0.5507 & -0.5732 & -0.5807 \\
$0.4405+0.5737 \mathrm{i}$ & $0.4428+0.6221 \mathrm{i}$ & $0.4418+0.6474 \mathrm{i}$ & $0.4412+0.6557 \mathrm{i}$ \\
$0.4405-0.5737 \mathrm{i}$ & $0.4428-0.6221 \mathrm{i}$ & $0.4418-0.6474 \mathrm{i}$ & $0.4412-0.6557 \mathrm{i}$ \\
0.7841 & 0.7711 & 0.7655 & 0.7638 \\
\hline
\end{tabular}

\subsection{System stability}

A study of the impact of the weighting factor, prediction horizon and control horizon in the system stability of the closed loop pole has been made. The weighting factor allows taking into account the minimization of the control increment as a criterion in the cost function besides the output error. If $\lambda$ is equal to zero or too small, the DMC would calculate a $\Delta u$ that only minimize the output error. This would lead to an aggressive control signal that could make the system unstable. Table 8 and Fig. 9 show the system poles for $\lambda=0, \operatorname{Pr}=100$ and several values of $M$.

The prediction and control horizon have also influence on stability. If the prediction horizon is too big, the errors term in the cost function would dominate it and the calculated control increments could be large enough to unstabilize the system. The effect of prediction horizon on stability can be seen in Table 9 and Fig. 9, as $\operatorname{Pr}$ grows the system becomes unstable.

Control and prediction horizons tend to unstabilize the system if they are given high enough values. This effect can be compen sated with the use of the weighting factor. When the value of $\lambda$ reaches the inflexion point of the curve shown in Fig. 6 high values of $M$ and $P r$ do not unstabilize the system. Tables 10 and 11 and Fig. 10 show that when a secure value of $\lambda$ has been reached, inadequate values of $M$ and $\operatorname{Pr}$ do not cause the system to go unstable.

\section{Preliminary design advices}

Taking into account the previous sections some useful conclu sions can be obtained:

- System response achieves lower oscillations when $T$ decreases and the prediction horizon is long enough.

- System response varies with Pr only up to a certain value, when the system reaches open loop behaviour. However, this effect can be corrected by an appropriate value of $M$.

- $n_{g}$ does not have influence once a certain value has been reached.

- $\lambda$ works in the opposite way to that of $\operatorname{Pr}$ as it tunes the DMC making a complex pole the dominant one but mitigating the complex part to eliminate oscillations.
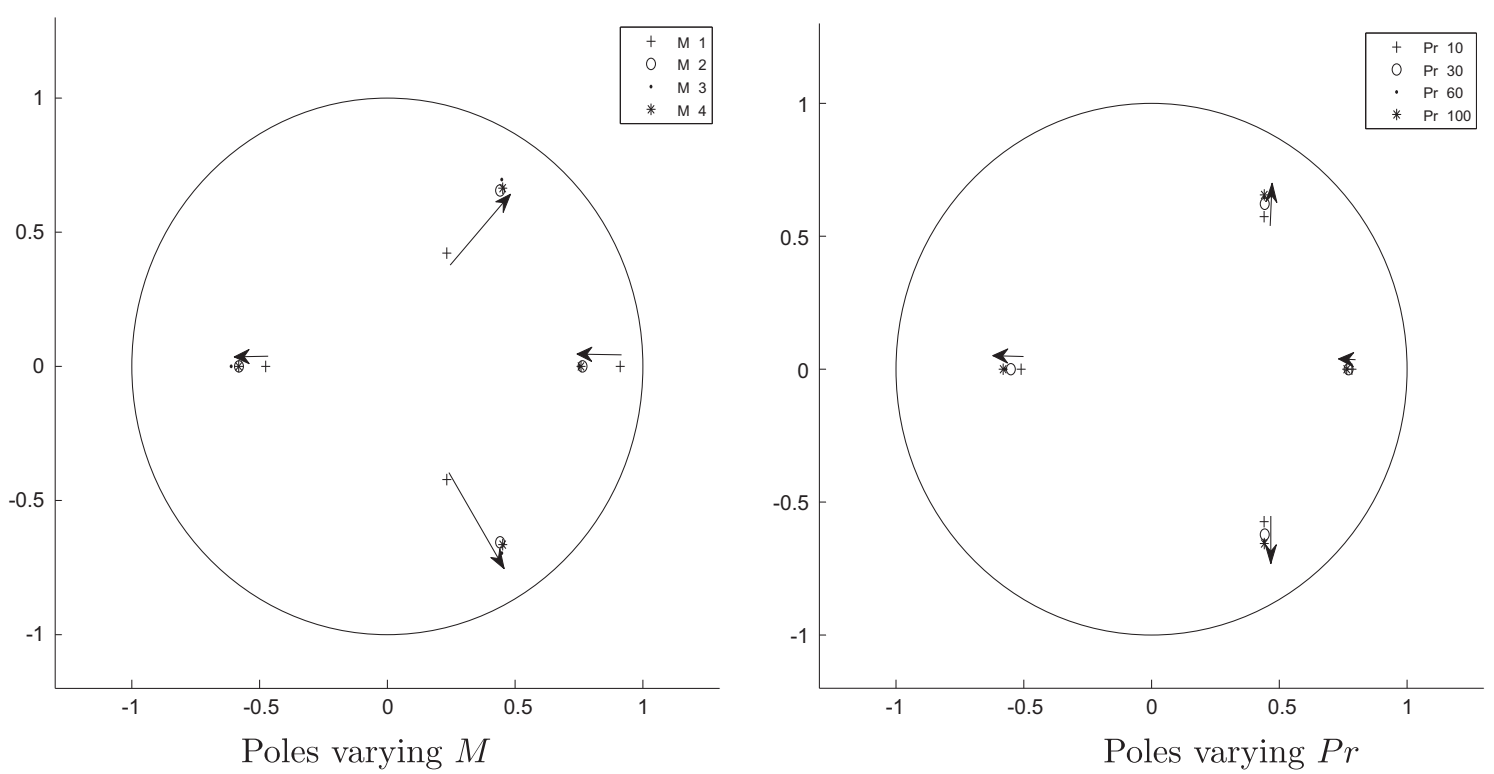

Fig. 10. Poles for $\lambda \quad 0.075$. 
- High values for $\operatorname{Pr}$ and $M$ along with too low values of $\lambda$ lead to system unstability.

So, the following tuning advices can be deduced:

1. $n_{g}=\operatorname{Pr}+n$, where $n$ is the required number of $g$ values required to reach the unit step.

2. $\operatorname{Pr}=\tau_{p}$ being the time constant of the FOPDT equivalent (time to reach $63 \%$ of step value).

3. $M$ has little influence on the process for small values of $\mathrm{Pr}$ (lower than $\tau_{p}$ ) but corrects the open loop behaviour when $\mathrm{Pr}$ is too high (greater than $\tau_{p}$ ). Also, according to Rawlings and Muske [10], this value should be greater than or equal to the number of unstable modes in the system to guarantee stability. 4. To calculate the sample time it will be taken into account the considerations of [1] who propose to take $0.1 \tau_{p}$. This has been proven to give an accurate result. This value will be considered as a maximum.

5. $\lambda$ contributes to soften response oscillations. But it has been observed that this effect disappears when prediction horizon becomes high enough. The reason is in the cost function minimized to obtain the DMC result (Eq. (13)). This function has two
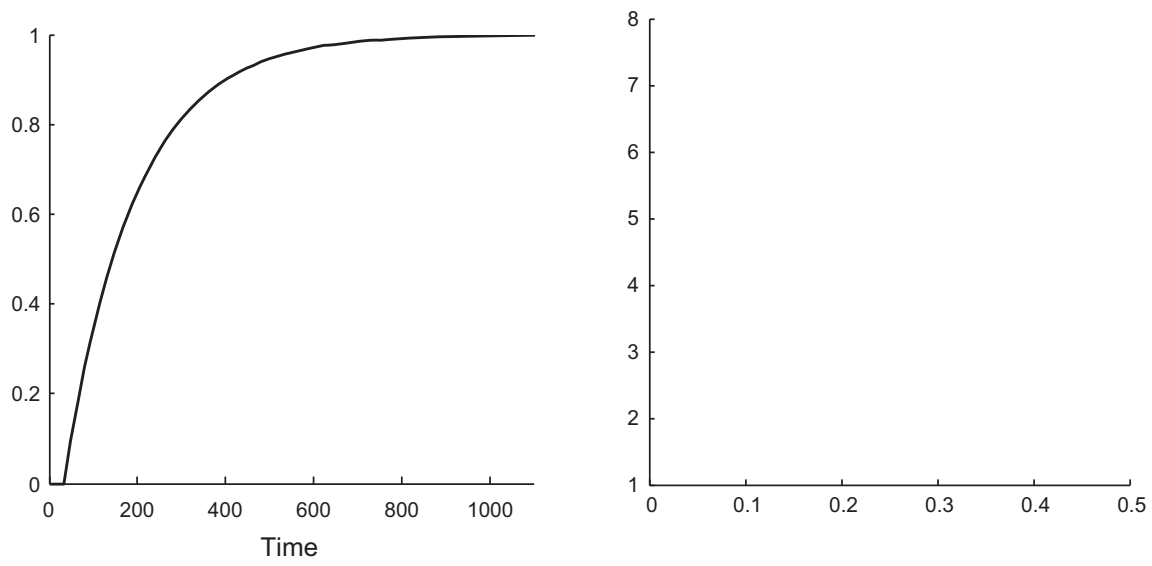
terms. The first one (depending on the prediction horizon) is the mean quadratic error and the second one (depending on the control horizon) are the control increments. $\lambda$ influence is limited to the second term which depends on the control horizon that usually has a smaller value than the prediction horizon (the solution of the DMC is given in Eq. (16)).

6. $\lambda$ has also an important effect on preventing system unstability and must take an appropriate value: the inflexion point of the first control increment curve.

\section{Validation of the method}

To validate the results from previous paragraphs the conclusions from Section 4 will be followed to tune the following benchmarks (extracted from [1]), but this time without the simplification done before.

Table 12

Tuning results for benchmark.

\begin{tabular}{llllllr}
\hline Benchmark & Time constant & Settling time & $\tau$ & $\operatorname{Pr}$ & $M$ & $n_{g}$ \\
\hline 1 & 160 & 1000 & 16 & 10 & 2 & 73 \\
2 & 180 & 1100 & 18 & 10 & 2 & 73 \\
3 & 180 & 800 & 18 & 10 & 2 & 100 \\
4 & 220 & 500 & 22 & 10 & 2 & 33 \\
5 & 250 & 1100 & 25 & 10 & 2 & 54 \\
\hline
\end{tabular}

(a)(4)

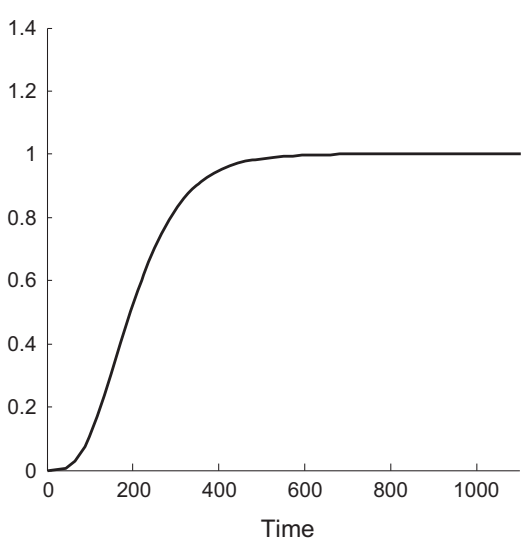

(a)(5)

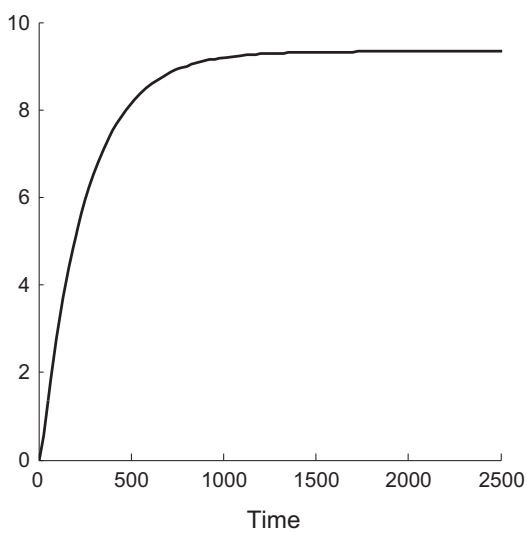

(b)(4)

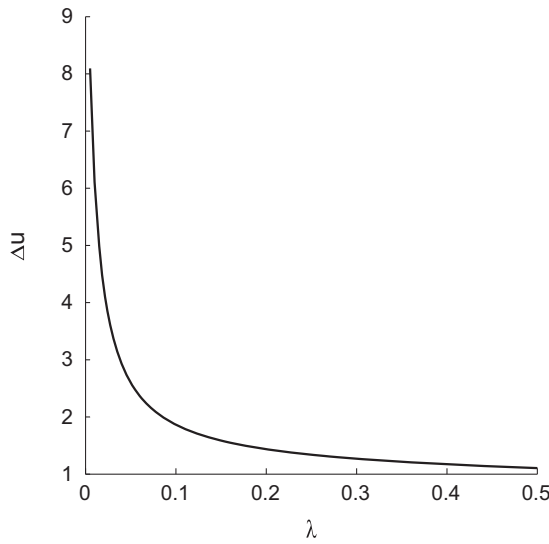

(b)(5)

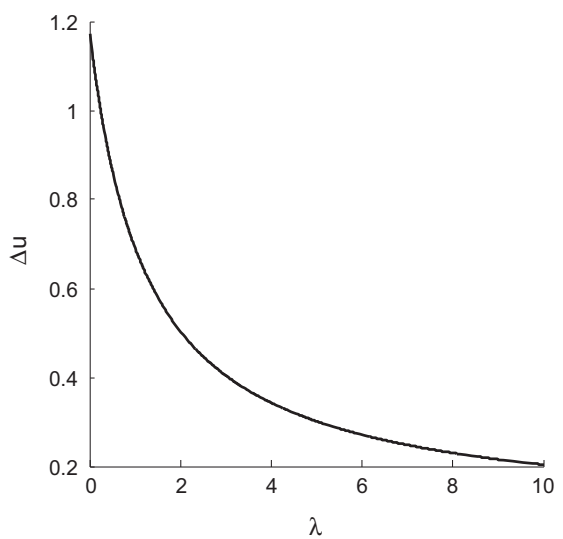

(c)(4)

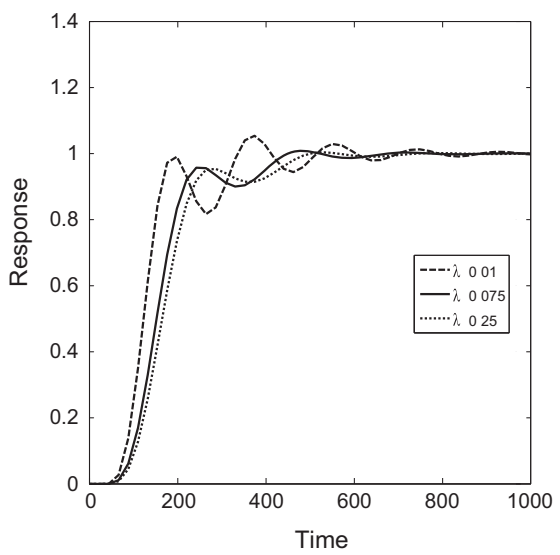

(c)(5)

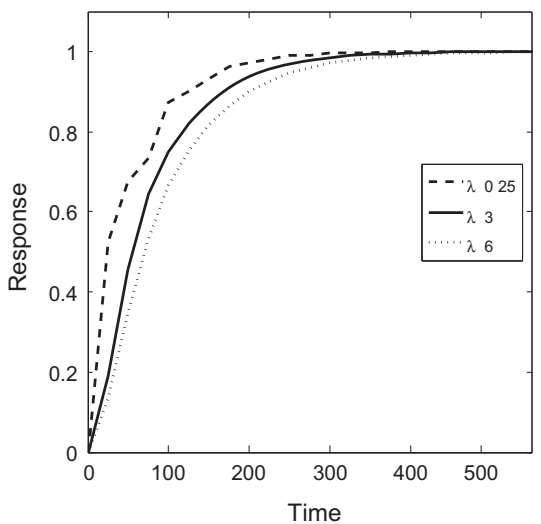

Fig. 12. (a) Step response of example, (b) weighting factor curve and (c) response of example. 
Benchmark 1:

$$
G(s)=\frac{e^{-50 s}}{(150 s+1)(25 s+1)}
$$

Benchmark 2:

$$
G(s)=\frac{e^{(1-50) e^{10 s}}}{(100 s+1)^{2}}
$$

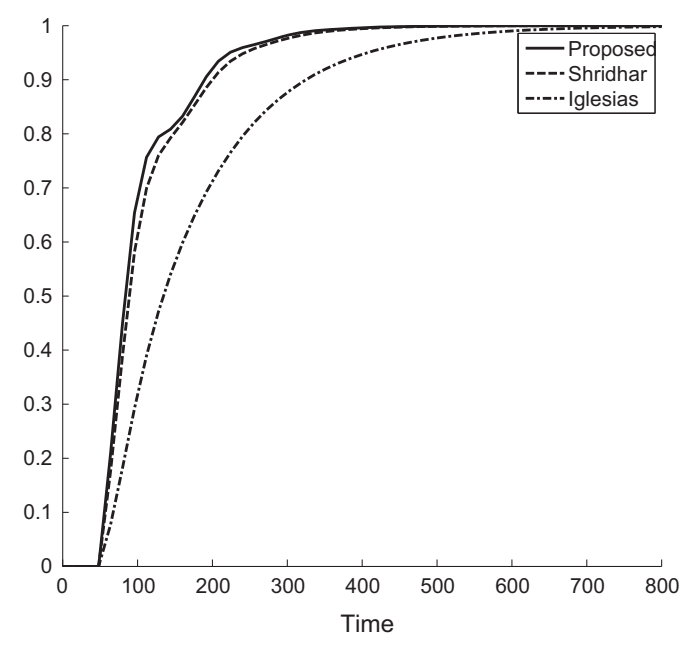

Process 1 comparison

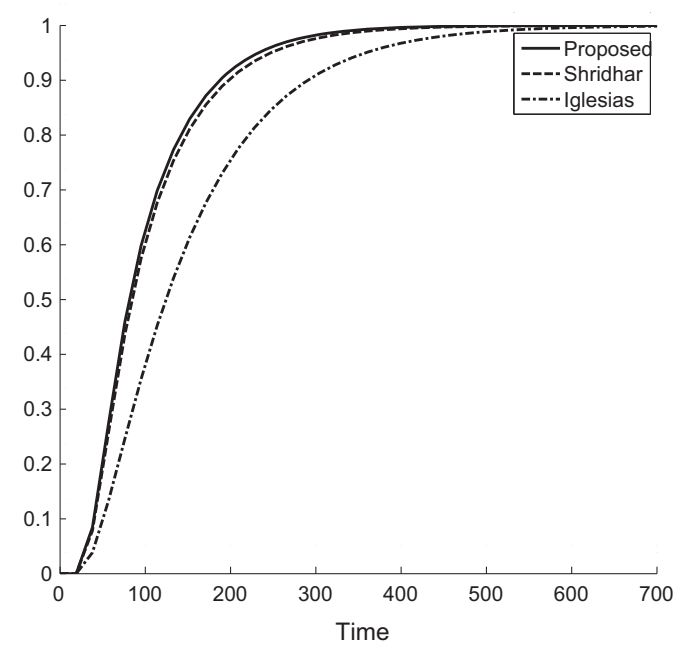

Process 3 comparison

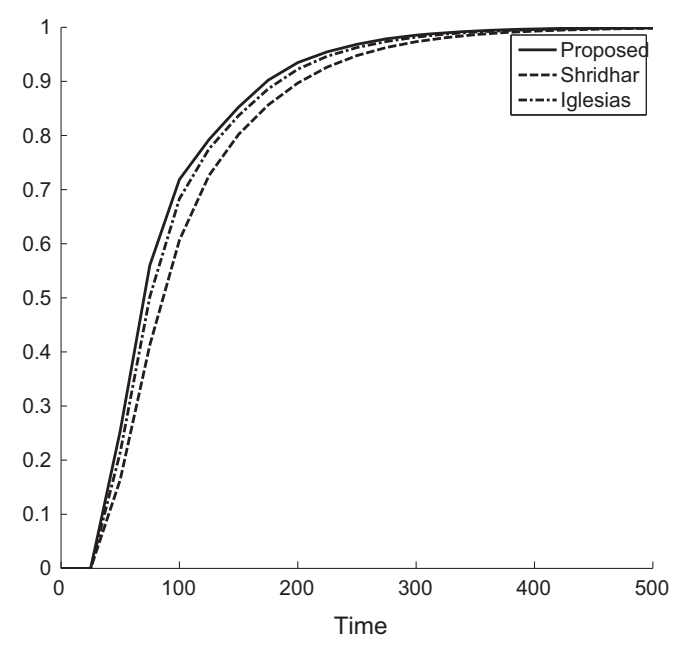

Process 5 comparison

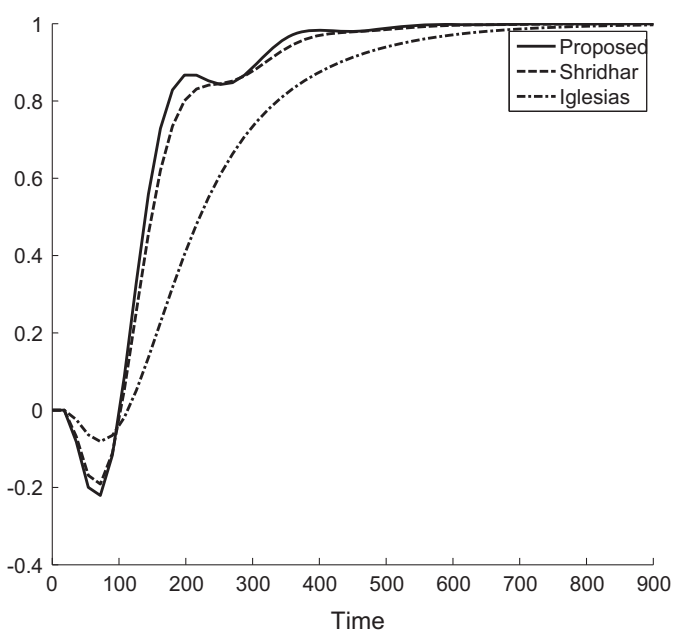

Process 2 comparison

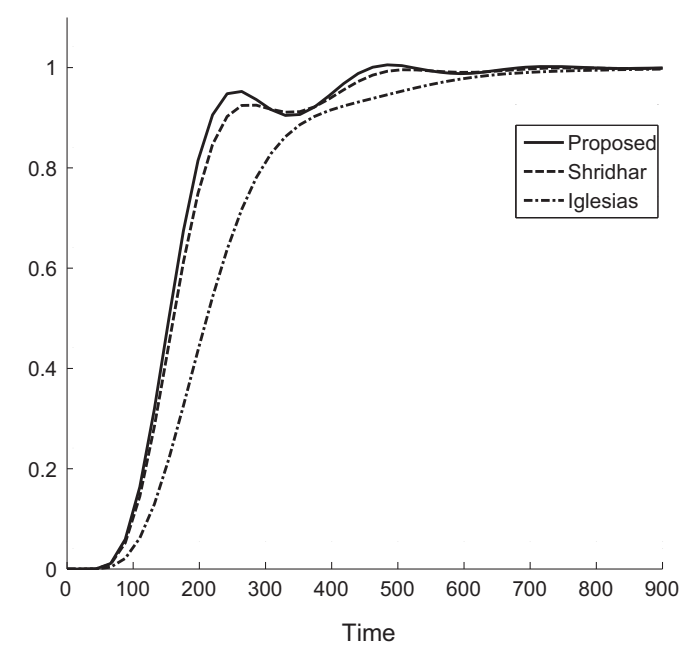

Process 4 comparison

Fig. 13. Comparison of proposed method. 
Benchmark 3:

$G(s)=\frac{e^{(1+50) e^{10 s}}}{(100 s+1)^{2}}$

Benchmark 4:

$G(s)=\frac{e^{-10 s}}{(50 s+1)^{4}}$

Benchmark 5: this process is extracted from [9, Section 4]:

$G(s)=\frac{0.0039 s+0.0038}{s^{2}+0.075 s+0.0003}$

Apart from its transfer function, each benchmark is character ized by its unit step response (required to obtain the time constant and settling time) and a graph showing the relationship between $\lambda$ and the first $\Delta u$, that allows us to obtain the correct range of values for $\lambda$.

\subsection{Results}

From the data presented in the previous section and the tuning rules for Section 4, the DMC parameters can be calculated for each benchmark. Those results are presented in Table 12 .

According to the above conclusions benchmarks should be tuned using a prediction horizon lower than 10 (taking into account that sampling time is $0,1 \tau_{p}$ ). To select a suitable weight ing factor the curve with the first control increment will be used. As it can be seen in Fig. 11 (using benchmark 1 as an example), the value of the first control increment changes very slowly once a value of $\lambda=0.25$ has been reached. This is the maximum useful value, higher values will only slowdown the process. For $\lambda=0$ the first control increment reaches values up to 6.5. Taking into account that the system gain and the setpoint are 1 , this would mean an unstable process. The first part of the curve goes from 0 to the inflexion point of the curve. In this part of the curve, $\lambda$ has a strong influence on the time response. Once the inflexion point has been surpassed, the weighting factor will have only a small influence. This inflexion point then becomes the maximum useful value.

It can also be seen how the first four $\lambda$ curves are different from the last one. The reason is that the first four benchmarks have the same gain, 1 , while benchmark 5 has a gain of nearly 10 . This means that the main factor that has influence on the shape of this curve is the system gain. The system gain is the factor to take into account when $\lambda$ is calculated. This is in accordance with the method of Shridhar and Cooper that calculates the weighting factor from the system gain [1].

As it is logical to think, Figs. 11 and 12 also show that the higher the system gain is, the higher the $\lambda$ must be.

\section{Comparison with other methods}

Despite the objective of this paper is not to improve other existing tuning methods but to offer an easy to use set of rules for a quick and reliable start up, these proposed tuning rules will be now compared with two methods extracted from the bibliography and mentioned in Section 1: The method of Shridhar Cooper [1] and the method of Iglesias et al. [4].

The method of Shridhar Cooper is composed of the following steps:

1. Approximate the system by a FOPDT:

$$
\frac{y(s)}{u(s)}=\frac{K_{p} e^{\Theta_{p} s}}{\tau_{p} s+1}
$$

2. Select, if possible, a sample time $T$ that fulfils $\left(\tau_{p}\right.$ is the FOPDT time constant and $\Theta_{p}$ is the system time delay)

$T \leq 0,1 \tau_{p} T \leq 0,5 \Theta_{p}$

3. Calculate the discrete delay:

$$
k=\frac{\Theta_{p}}{T}+1
$$

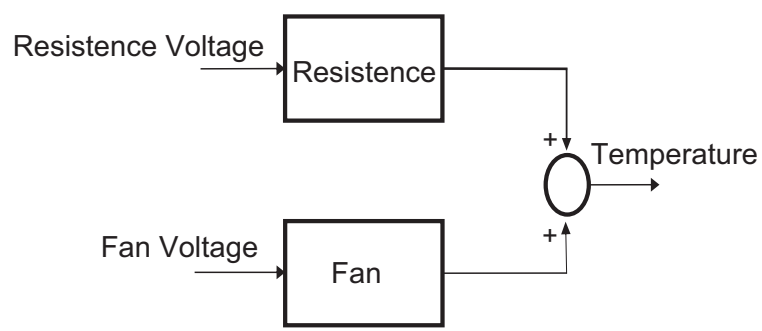

Fig. 15. Thermal plant block diagram.

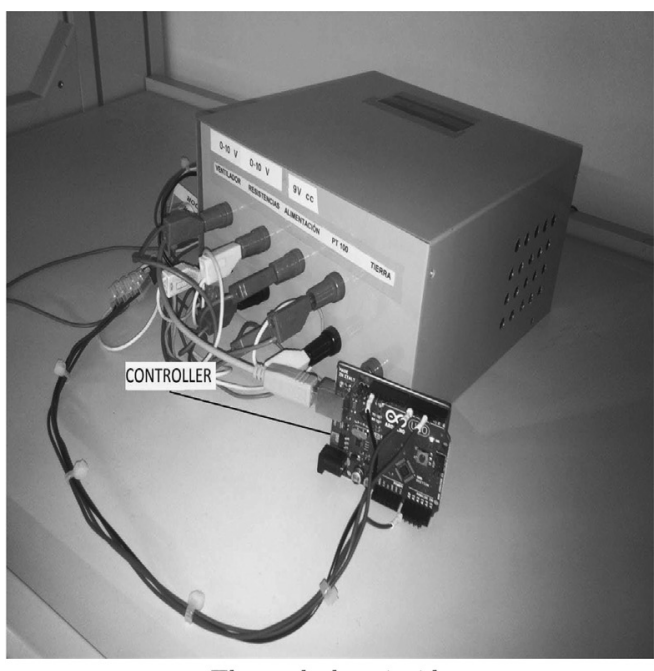

Thermal plant inside

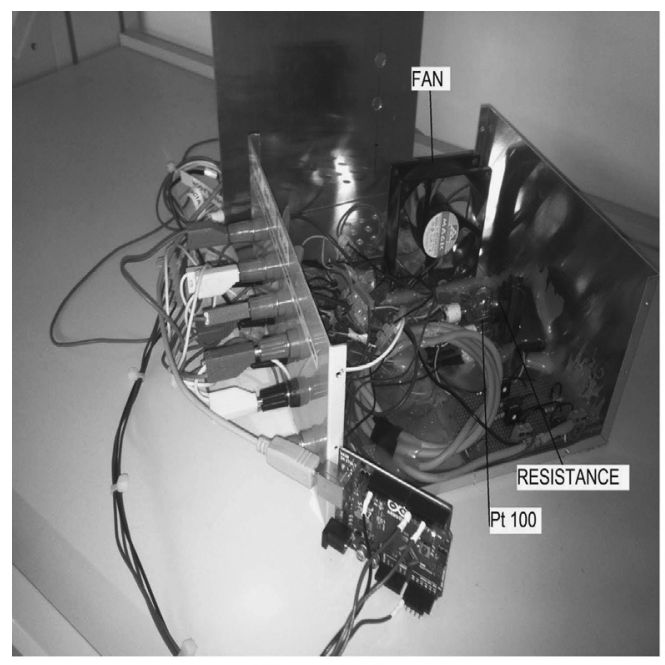

Thermal plant

Fig. 14. Thermal plant. 
4. Calculate the model and prediction horizons:

$$
\operatorname{Pr}=n_{g}=5 \frac{\tau_{p}}{T}+k
$$

5. Select the control horizon and the weighting factor:

$$
f=0 ; \quad M=1, \quad f=\frac{M}{500}\left(\frac{3,5 \tau_{p}}{T}+2 \quad \frac{M 1}{2}\right) ; \quad M \geq 2 \lambda=f K_{p}^{2}
$$

Iglesias et al. propose an equation based on statistical methods that calculate the weighting factor. The other parameters must be calculated using other method. In this case, the method of Shridhar Cooper will be used:

$\lambda=1.631 K_{p}\left(\frac{\Theta}{\tau}\right)^{0.4094}$

where $K_{p}$ is the system gain, $\Theta$ is the system delay and $\tau$ is the FOPDT time constant.

Table 13 shows the calculated parameter for each method. Fig. 13 shows the comparison of time responses for the three methods.

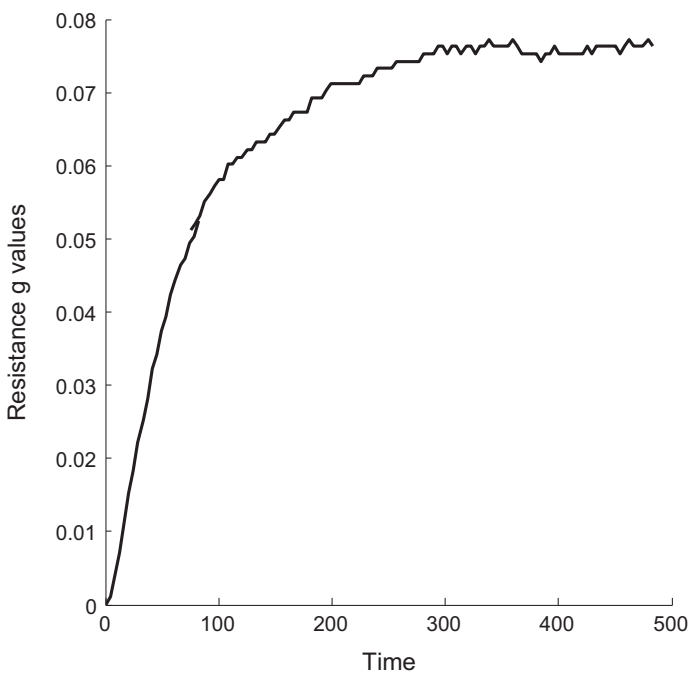

Resistance step response

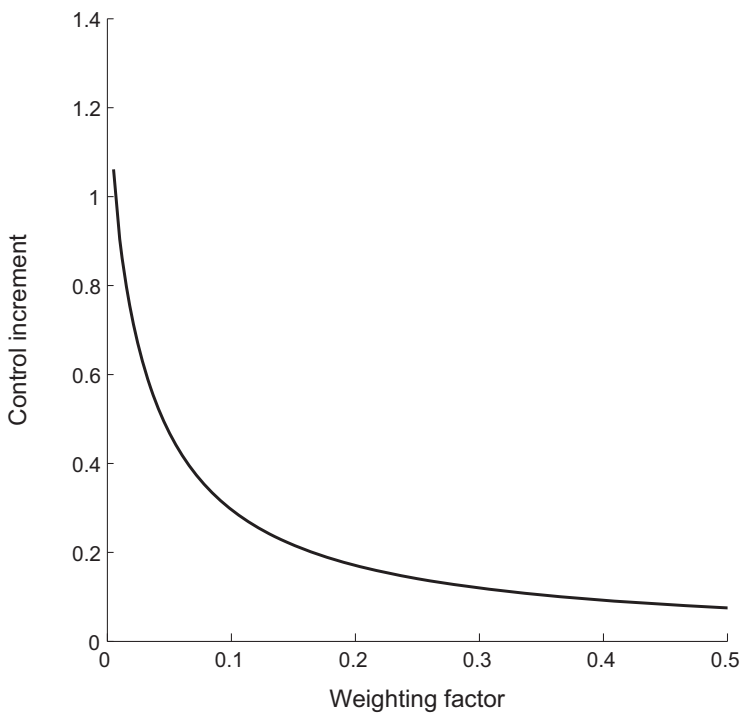

Resistance weighting factor curve

\section{Testing on a thermal plant}

The final exam for the design rules obtained in this paper will be a test on a real system. This system is thermal plant with two inputs and one output. The plant is basically composed of (see Fig. 14)

- a fan to decrease temperature,

- a set of resistances (lightbulbs) that will increase temperature,

- a Pt100 to measure temperature.

Table 14

Thermal plant results.

\begin{tabular}{lllll}
\hline Case & $\lambda$ & $n_{g}$ & $\operatorname{Pr}$ & $M$ \\
\hline 1 & 0.2 & 101 & 20 & 2 \\
2 & 0.1 & 101 & 20 & 2 \\
3 & 0.01 & 101 & 20 & 2 \\
4 & 0.1 & 101 & 20 & 1 \\
5 & 0.1 & 101 & 20 & 5 \\
6 & 0.1 & 101 & 10 & 2 \\
\hline
\end{tabular}

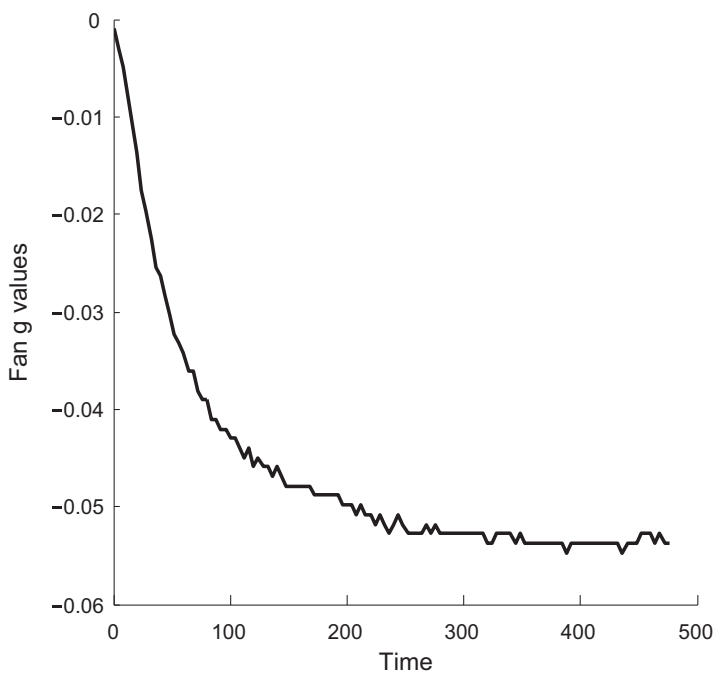

Fan step response

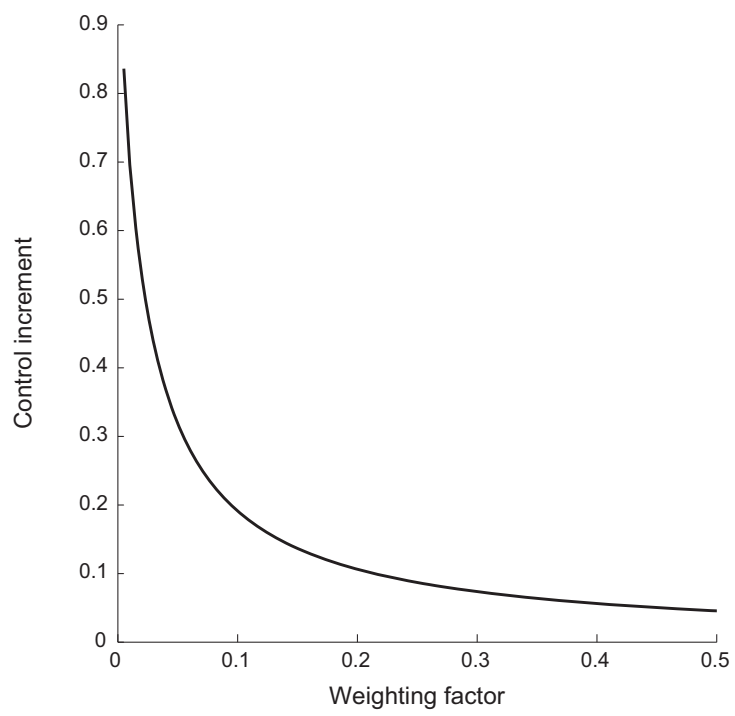

Fan weighting factor curve

Fig. 16. Thermal plant definition graphs. 
In this system (Fig. 15) the temperature inside the plant (out put) is controlled by the voltage of the fan and resistances (outputs).

In Fig. 16 the step response of the system can be seen. The time constants for the fan and resistances are

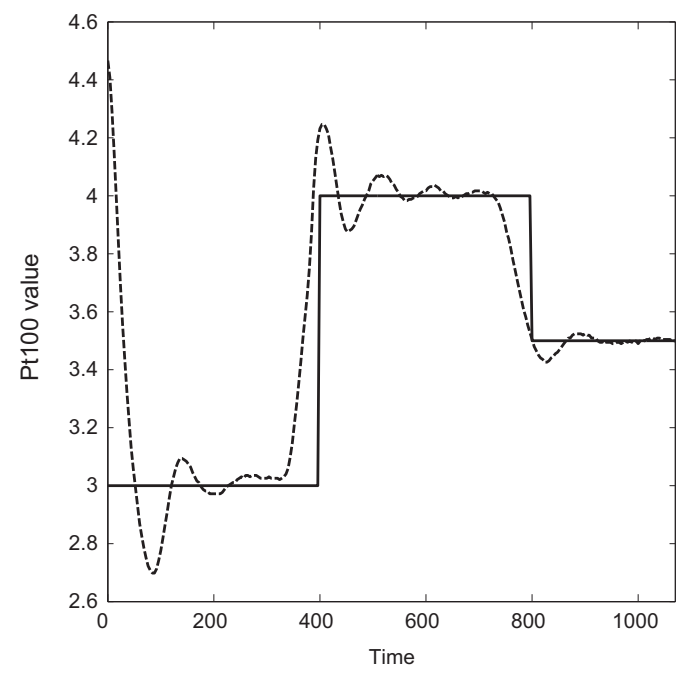

Case 1

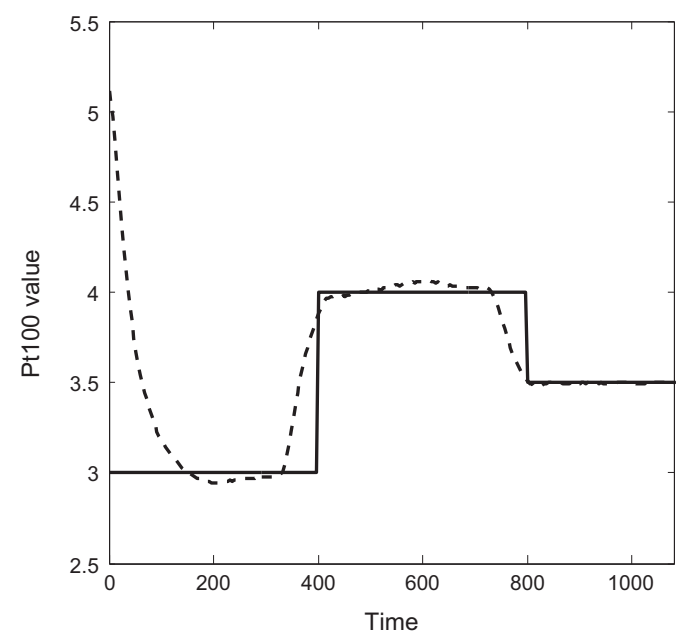

Case 3

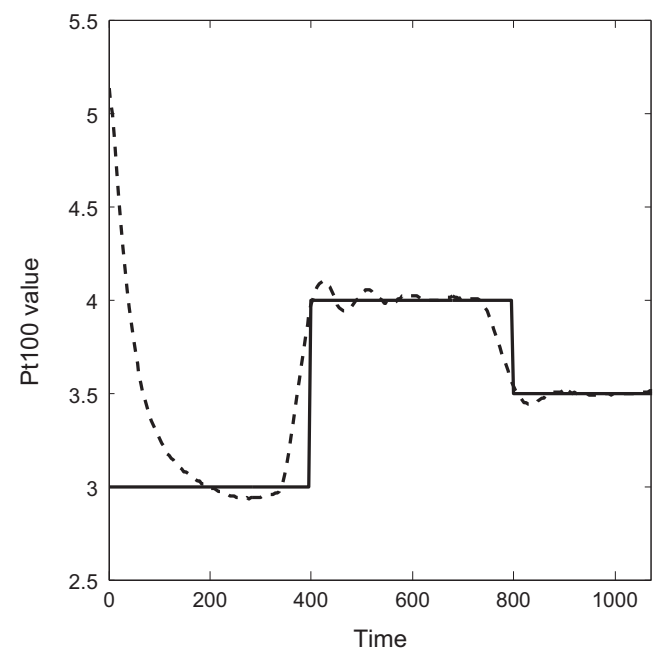

Case 5
- $76 \mathrm{~s}$ for the resistance,

- $64 \mathrm{~s}$ for the fan.

Taking into account that the step time is $4 \mathrm{~s}$ (set by the system) this means that the prediction horizon is 20 . To make the study

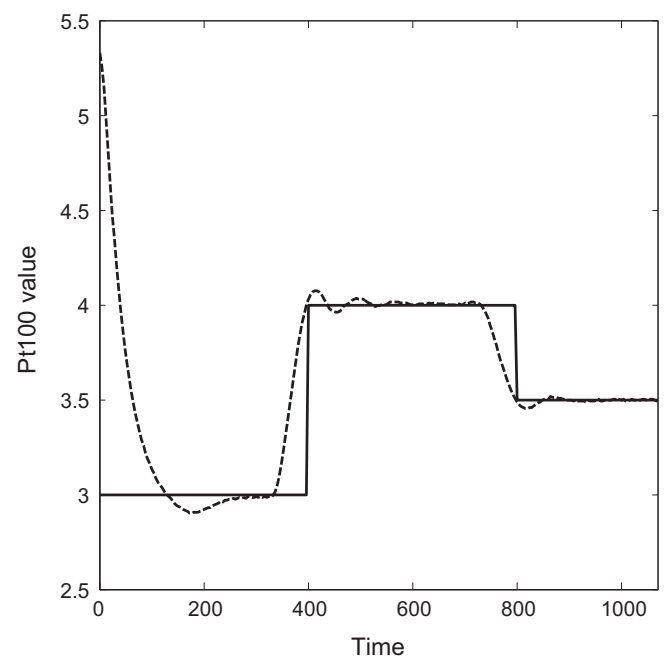

Case 2

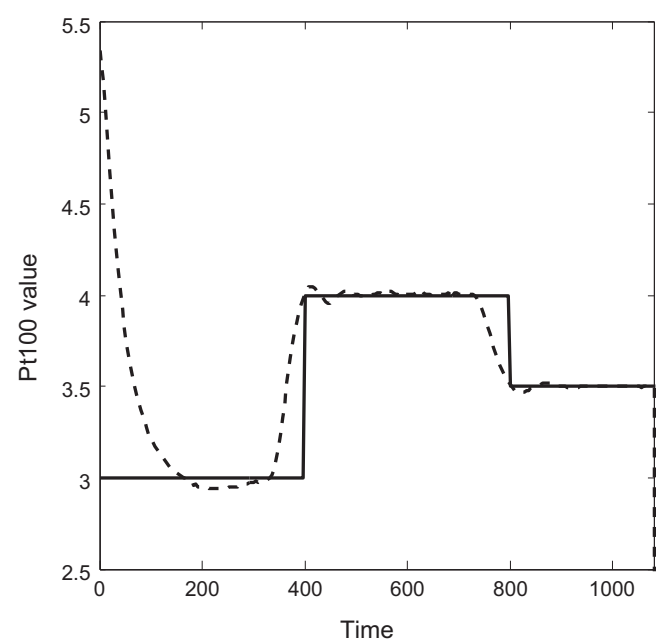

Case 4

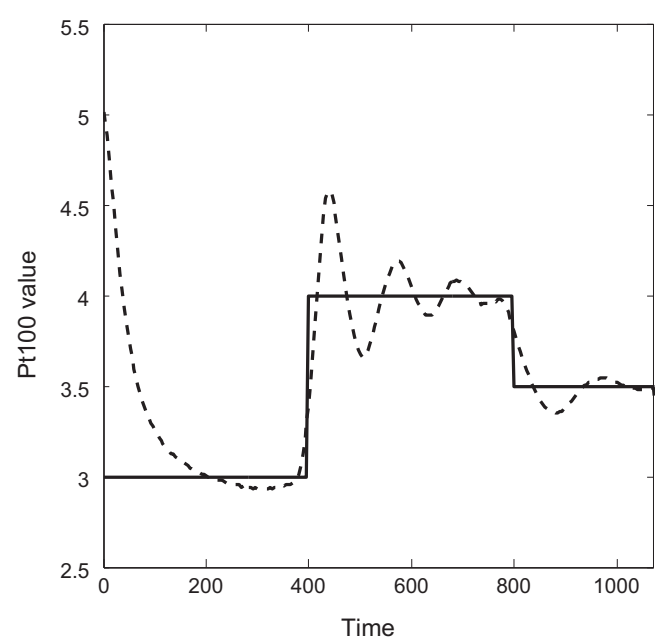

Case 6

Fig. 17. Results of thermal plant. The continuous line shows the setpoint to be followed and the dashed line the system response. 
easier we will use the same prediction horizon for both inputs. Model horizon will be 100 according to Section 3.4. Control horizon will be set to a small value, 2 . To select the weighting factor two curves comparing the first control increment with $\lambda$ are drawn (Fig. 16). Both curves show that in this plant $\lambda$ has only useful values up to 0.2. Bigger values will provide very small control increments and, consequently, a slow process. Moreover, too high values for $\lambda$ will make that the system not to handle the thermal inertia.

The explanation of cases is as follows (see Table 14 and Fig. 17):

- Case 1: This is the consequence of a too high value of $\lambda$. As explained in Section 3.3 this value has made the complex poles dominant, resulting in an oscillating system. This inadequate value slows down the fan and resistance actions and makes them more difficult to defeat the thermal inertia.

- Case 2: Better behaviour with only reducing $\lambda$ can be seen. This gives more capability to the fan and resistance to deal with the thermal inertia by allowing bigger control increments. From the point of view of Section 3.3, a smaller value of $\lambda$ weakens the effect of complex poles.

- Case 3: This response shows the effect of a minimum $\lambda$. In this process it only means a small overpeak.

- Case 4: The difference between this result and case 2 is hard to differentiate. The only difference between them is the value of $M$ and this parameter only shows a strong influence if $\mathrm{Pr}$ reaches high values.

- Case 5: The effect of a too high value of $M$ can be seen. In Section 3.2 it was explained that the influence of complex poles grows with $M$ as well as with $\lambda$.

- Case 6: This response shows the effect of a too small Pr. The DMC algorithm has no enough data to perform accurate predictions. In Section 3.2 it was explained that a too small Pr makes the complex poles to become dominant.

\section{Conclusions}

In this paper some design rules for the DMC tuning are obtained. To this aim, a study of the effect of the main DMC parameters on the time response has been made. The controlled system is transformed to a Linear Time Invariant one in order to compute the closed loop poles that determine the system dynamics. A First Order Plus Dead Time approximation has been used to reduce the number of the existing poles and make the analysis easier. The closed loop poles movement study is traduced to prediction horizon, control horizon, weighting factor and sample time parameters. This yields to some rules to be followed by practitioners to a quick start procedure.

The application of this guide will not provide the optimal solution, but ensure an acceptable and stable control and the parameters set obtained can be used as a good starting point prior to make adjustments. The results of the method over a set of simulation benchmarks and a real plant have been presented to prove the performance reached. A comparison of results with other tuning methods has also been made.

\section{Acknowledgements}

The authors would like to thank the anonymous reviewers for their helpful comments and suggestions.

\section{References}

[1] Shridhar Rahul, Cooper Douglas J. A tuning strategy for unconstrained SISO model predictive control. Ind Eng Chem Res 1997:36(3):729-46.

[2] Trierweiler JO, Farina LA. RPN tuning strategy for model predictive control. J Process Control 2003;13(7):591-8.

[3] Han Kai, Zhao Jun, Qian Jixin. A novel robust tuning strategy for mode preditive control. In: The 6th world congress on intelligent control and automation. WCICA 2006, vol. 2, 2006. p. 6406-10.

[4] Iglesias Edinzo J, Sanjuan Marco E, Smith Carlos A. Tuning equation for dynamic matrix control in SISO loops. Ingeniería y Desarollo 2006;19:88-100.

[5] Rossiter JA. Model based predictive control. A practical approach. Boca Raton, FL: (Chemical Rubber Company) CRC Press; 2003.

[6] Camacho EF, Bordons C. Model predictive control. 2nd ed.. London: SpringerVerlag; 2003.

[8] Valencia-Palomo G, Rossiter JA. Auto tuned predictive control based on minimal plant information. In: 7th IFAC international symposium on advanced control of chemical processes, 2009.

[9] Sheng Jie, Chen Tongwen, Shah Sirish L. Generalized predictive control for non uniformly sampled systems. J Process Control, 2002, 12:875-85.

[10] Rawlings J, Muske K. The stability of constrained receding horizon control. IEEE Trans Autom Control 1993;38(October (10)).

[11] Garriga Jorge L, Soroush Masoud. Model predictive controller tuning via eigenvalue placement. In: American control conference, 2008.

[12] Cutler CR, Ramaker BL. Dynamic matrix control-a computer control algorithm. In: Proceeedings of JACC, San Francisco, USA, 1980.

[13] Ziegler JG, Nichols NB. Optimum settings for automatic controllers. Trans ASME 1942, November, pp. 759-68.

[14] Kokate RD, Waghmare LM. Review of tuning methods of DMC and performance evaluation with PID algorithms on a FOPDT model. Int J Control Autom 2011:4(June (2)).

[16] Ahmad A, Wahid A. Application of model predictive control (MPC) tuning strategy in multivariable control of distillation column. Reaktor 2007;11 (December (2)):66-70.

[17] Bagheri Peyman, Khaki-Sedigh Ali. Tuning of dynamic matrix controller for FOPDT models using analysis of variance. In: 18th IFAC world congress, Milano, Italy, August 28-September 2, 2011.

[18] Wojsznis Willy, Gudaz John, Blevins Terry, Mehta Ashish. Practical approach to tuning MPC. ISA Trans 2003;42:149-62. 